\title{
Synthesis of Chiral Donor-Acceptor Dyes to Study Electron Transfer Across a Chiral Bridge
}

Marc J. Junge, ${ }^{[a]}$ Mike A. Kordan, ${ }^{[a]}$ and Erin T. Chernick ${ }^{*[b, a]}$

${ }^{[a]}$ Institut für Organische Chemie, Universität Tübingen, Auf Der Morgenstelle 18, 72076 Tübingen, Germany.

${ }^{[b]}$ Department of Chemistry, University of Victoria, PO Box 1700 STN CSC Victoria, BC, V8W 2Y2, Canada.

*E-Mail address: erinchernick@ uvic.ca

Supporting Information 


\section{Table of contents}

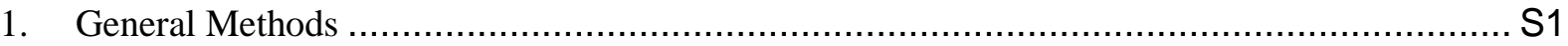

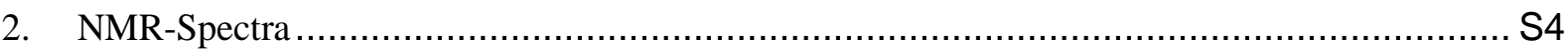

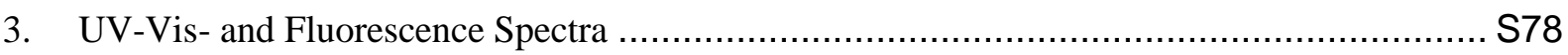

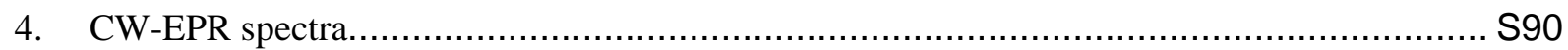

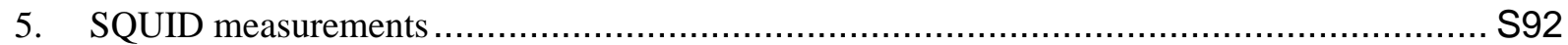

6. ESI/APCI-High Resolution Mass Spectrum ................................................................ S93

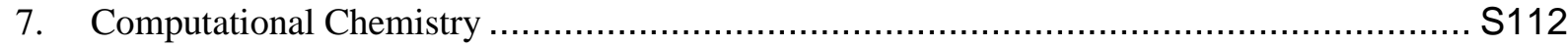

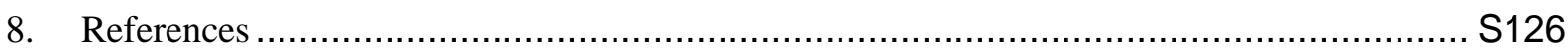




\section{General Methods}

Solvents for chromatography and workup including technical grade diethyl ether, ethyl acetate (EtOAc), hexane, and dichloromethane (DCM) were distilled prior to use with a rotary evaporator or purchased as a high performance liquid chromatography (HPLC) grade. DCM, hexane, tetrahydrofuran (THF), and toluene used for the synthetic reactions were purified by an SPS system (MBraun MB-SPS-800). DMF, $n$-BuLi (2.5 M in hexane), and TMEDA used in the synthetic reactions were bought anhydrous and under inert atmosphere from Sigma-Aldrich. Triethylamine was distilled over $\mathrm{CaH}$ under a $\mathrm{N}_{2}$ atmosphere prior to use. Methanol for recrystallization and synthetic reactions was purchased as an HPLC grade. Concentrated $\mathrm{HCl}_{(\mathrm{aq})}$ was used as a technical grade and diluted to $1 \mathrm{M}$ concentration. 4-Amino2,2,6,6-tetramethylpiperidinyloxyl free radical (TEMPO-amine), $\mathrm{Al}_{2} \mathrm{O}_{3}$, 9-(4-bromophenyl)9H-carbazole (2a), $\mathrm{CuI}, \mathrm{CsF}, \mathrm{CsCO}_{3}$, ethyl cyanoacetate, 1-fluoro-4-iodobenzene, piperidine, $\mathrm{I}_{2}, \quad \mathrm{~K}_{2} \mathrm{CO}_{3}, \quad \mathrm{PdCl}_{2}$, tetra- $n$-butylammonium fluoride (TBAF), tributyltin chloride, tri-isopropylsilylacetylene, trimethylsilylacetylene, and tri-t-butylphosphine were used without further purification. DCM- $d_{2}$ and $\mathrm{CDCl}_{3}$ were purified directly before use by passing through a basic alumina column. Solvent impurities and water were not fully removed by this technique, leading to impurities. ${ }^{1} \mathrm{H}-\mathrm{NMR}$ gave signals at 1.52 (water), 1.26 (grease), and 0.08 (grease) ppm. $\quad{ }^{13} \mathrm{C} \mathrm{NMR} \quad$ gave $\quad$ signals $\quad$ at $\quad 1.2$ (grease) ppm. ${ }^{[1]}$ Tetrakis(triphenylphosphine)palladium(0), ${ }^{[2]}$ tributyl(3-methylthiophene-2-yl)stannane (3), ${ }^{[3]}$ 4,7-dibromobenzo $[c][1,2,5]$ thiadiazole $(\mathbf{1 0}),{ }^{[4]} \quad 3,6$-dibromo- $9 H$-carbazole $(\mathbf{1}),{ }^{[5]} \quad$ 5-(7bromobenzo[c][1,2,5] thiadiazol-4-yl)thiophene-2-carbaldehyde (8), ${ }^{[6]} \quad 3,3$ '-diiodo-2,2'dimethoxy-1,1'-binaphthalene $(\mathbf{1 3}(\boldsymbol{S}) \mathbf{a}),{ }^{[7]}$ and $2,2^{\prime}$-dimethoxy-1,1'-binaphthalene $(\mathbf{1 2}(\boldsymbol{S}))^{[8]}$ were synthesized according to the reported literature. 3-Iodo-2,2'-dimethoxy-1,1'binaphthalene $(\mathbf{1 3}(S) b)$ was synthesized in a modified synthetic procedure of reported literature in improved yields. ${ }^{[9]}$

Thin-Layer Chromatography (TLC) was performed on silica TLC plates (Macherey-Nagel, Polygram Sil G/UV) and visualized by illumination with a UV lamp (254 and $365 \mathrm{~nm}$ wavelength). Silica gel (Macherey-Nagel, 0.04-0.063 mm) was used as received. Size exclusion chromatography was performed on Bio-Beads ${ }^{\mathrm{TM}} \mathrm{S}-\mathrm{X} 3$ Resin with toluene as an eluent.

All NMR spectra were recorded at $298 \mathrm{~K}$ on 400 and $700 \mathrm{MHz}$ Bruker Avance III spectrometers. ${ }^{1} \mathrm{H}$ and ${ }^{13} \mathrm{C}$ shifts were referenced to the residual solvent signal $\left(\mathrm{CD}_{2} \mathrm{Cl}_{2}\right.$, ${ }^{1} \mathrm{H}-\mathrm{NMR}: \delta=5.32 \mathrm{ppm},{ }^{13} \mathrm{C} \mathrm{NMR}: \delta=53.84 \mathrm{ppm}$ and $\mathrm{CDCl}_{3},{ }^{1} \mathrm{H}-\mathrm{NMR}: \delta=7.26 \mathrm{ppm}$, ${ }^{13} \mathrm{C}$ NMR: $\left.\delta=77.16 \mathrm{ppm}\right) .{ }^{[1]}$ Structural assignments were made with additional information 
from $\mathrm{gCOSY}$, gHSQC, gHMBC, and gNOESY experiments. Mass spectrometry measurements were recorded on a maXis 4G-UHR-TOF Bruker Daltonics spectrometer using the electrospray ionization (ESI) or the atmospheric-pressure chemical ionization (APCI) technique. UV/Vis spectra were recorded at room temperature in a $1 \mathrm{~cm}$ quartz cuvette using a Perkin-Elmer Lambda 1050 spectrophotometer. Fluorescence spectra were recorded on a PTI Quantamaster QM4, equipped with a $75 \mathrm{~W}$ steady-state xenon short arc lamp for excitation. The emission was recorded with a PTI P1.7R detector module covering UV-vis-NIR wavelength (200-1000 nm). When necessary, long pass filter glass plates (Schott, $3 \mathrm{~mm}$ thickness) were used in the emission path. The spectral selection was achieved by single-grating monochromators (excitation: 1200 grooves/mm, $300 \mathrm{~nm}$ blaze and UV-Vis emission: 1200 grooves $/ \mathrm{mm}$, $500 \mathrm{~nm}$ blaze). The samples were dissolved in HPLC grade solvents without further purification. An absorbance of 0.1 at the excitation wavelength was used without degassing. Fluorescence quantum yields were determined by the method introduced by Parker and Rees ${ }^{[10]}$ using the following equation:

$$
\Phi_{x}=\Phi_{R} * \frac{\operatorname{Grad}_{x}}{\operatorname{Grad}_{R}} * \frac{n_{x}^{2}}{n_{R}^{2}}
$$

Where $n$ is the refractive index (ACN: $n=1.344$, DCM: $n=1.424, \mathrm{H}_{2} \mathrm{O}: n=1.330$, $0.1 \mathrm{M} \mathrm{H}_{2} \mathrm{SO}_{4}: n=1.334$ ) and Grad is the linearly fitted slope from the plot of the integrated luminescence intensity versus the absorbance at the excitation wavelength. The subscripts ' $x$ ' and ' $R$ ' refer to the sample and the reference, respectively. Quinine sulfate in $0.1 \mathrm{M} \mathrm{H}_{2} \mathrm{SO}_{4}$ was used as a reference material, with a fluorescence quantum yield of $54.6 \%$, for $\mathbf{3 b}, \mathbf{4 a}, \mathbf{4 b}, \mathbf{6}, \mathbf{7}$, 14(S), and 20(S). ${ }^{[11]}$ Coumarin 153 in $\mathrm{ACN}$ was used as a reference material, with a fluorescence quantum yield of $43 \%$, for 9, 11a, 17(S)a, 18(S)a, and 19(S)a. ${ }^{[12]}$ Tris(2,2'-bipyridyl)dichlororuthenium(II) $* 6 \mathrm{H}_{2} \mathrm{O}$ in water was used as a reference material, with a fluorescence quantum yield of $2.8 \%$, for $17(S) \mathbf{b}, \mathbf{1 8}(S) \mathrm{b}, \mathbf{1 9}(S) \mathrm{b}, \mathbf{2 1}(S), \mathbf{2 2}(S)$, and 23(S). ${ }^{[13]}$ EPR measurements were performed on a Bruker ESP300E X-Band instrument, operating at a microwave frequency of $9.84 \mathrm{GHz}$. IR-spectra were recorded at $\mathrm{rt}$ using a Jasco FT/IR4100 spectrometer with an ATR application. Optical rotations were measured at a Jasco P-1020 at $295 \mathrm{~K}$ and a concentration of 0.1 and 0.01 in DCM. Melting points were measured with a Büchi Melting Point B540.

Calculations were performed using the Gaussian16 package. ${ }^{[14]}$ Geometries were optimized in the gas phase using the B3LYP ${ }^{[15]}$ functional and the $6-31 \mathrm{G}^{*}{ }^{[16]}$ basis set for all atoms. Frequency analyses were performed at the same level of theory to conform that the obtained geometries correspond to minima. No imaginary frequencies were found. 
SQUID measurements were performed on a Quantum Design spectrometer, equipped with a 1822- MPMS controller, a 1802 R/G bridge, a Model 2000 VHF SQUID amplifier, and a Model 2010 SQUID controller. The magnetic susceptibility was measured between 5 and $300 \mathrm{~K}$. The molar magnetic susceptibility $\chi_{\text {mol }}$ was calculated by the Curie-Weiss law, where $C$ is the Curie constant, $T$ is the temperature in $\mathrm{K}$ and $\theta$ is the Weiss constant. The equation was expanded with a temperature-independent correction factor $\chi_{0}$, for the diamagnetic correction and the diamagnetic probe container. ${ }^{[17]}$

$$
\chi_{m o l}=\frac{C}{T-\theta}+\chi_{0}
$$

The experimental Bohr magneton $\mu_{\text {exp }}$ was calculated by $\mu_{\text {exp }}=2.83 * \sqrt{C}$. The amount of unpaired spin was calculated by the spin-only formula, where $g=2.00023$ is the gyromagnetic ratio for a free electron and $S$ is the number of unpaired electrons. ${ }^{[17]}$

$$
\mu_{\text {exp }}=g \sqrt{S(S+1)}
$$

Reactions under $\mathrm{N}_{2}$ atmosphere were carried out in oven-dried glassware, that was evacuated and refilled with $\mathrm{N}_{2} 3$ times. 


\section{NMR-Spectra}

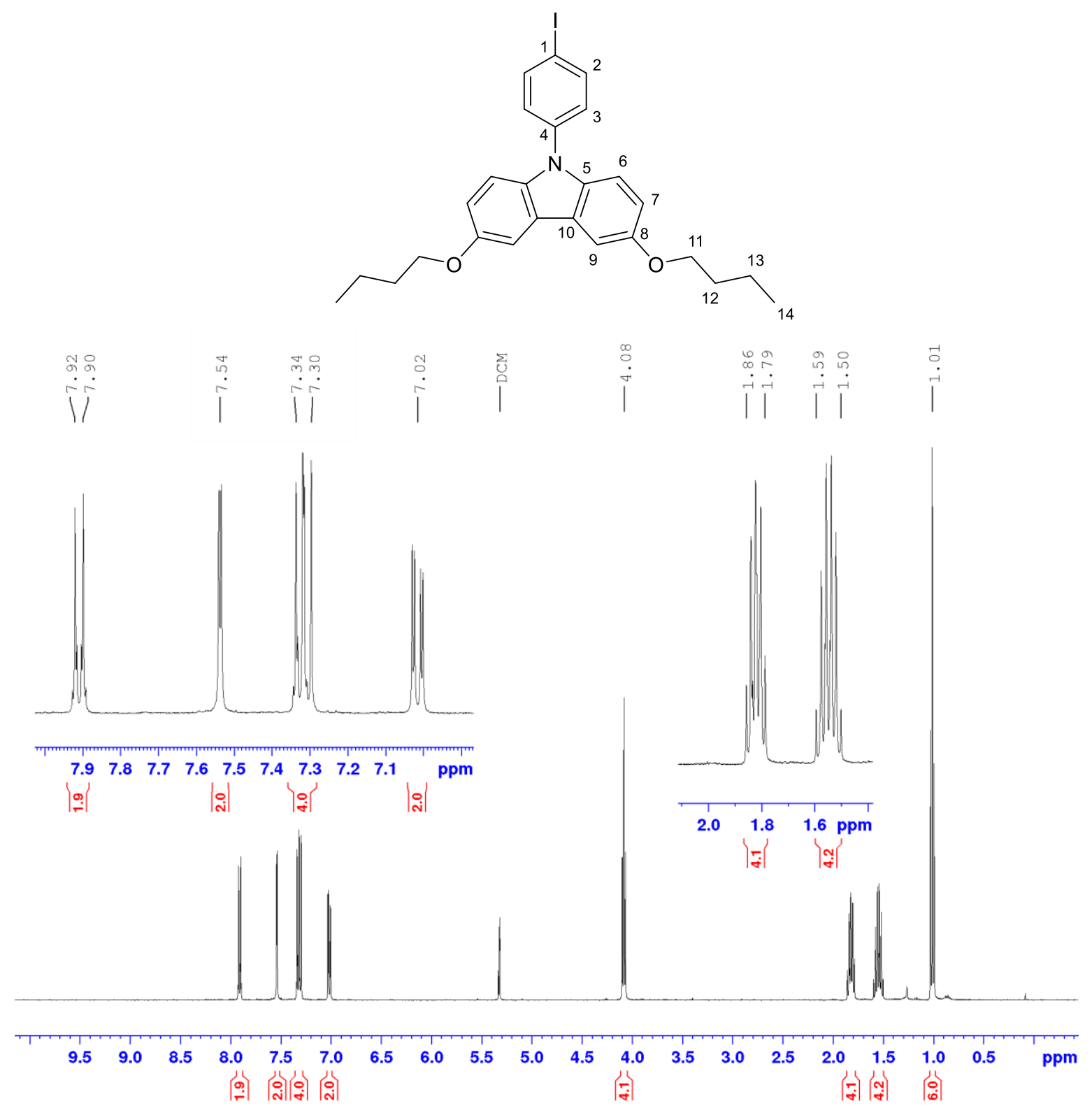

Figure S1. ${ }^{1} \mathrm{H} \mathrm{NMR}$ of $\mathbf{2 b}$ in $\mathrm{CD}_{2} \mathrm{Cl}_{2}$ at a $400 \mathrm{MHz}$ spectrometer at $298 \mathrm{~K}$. 


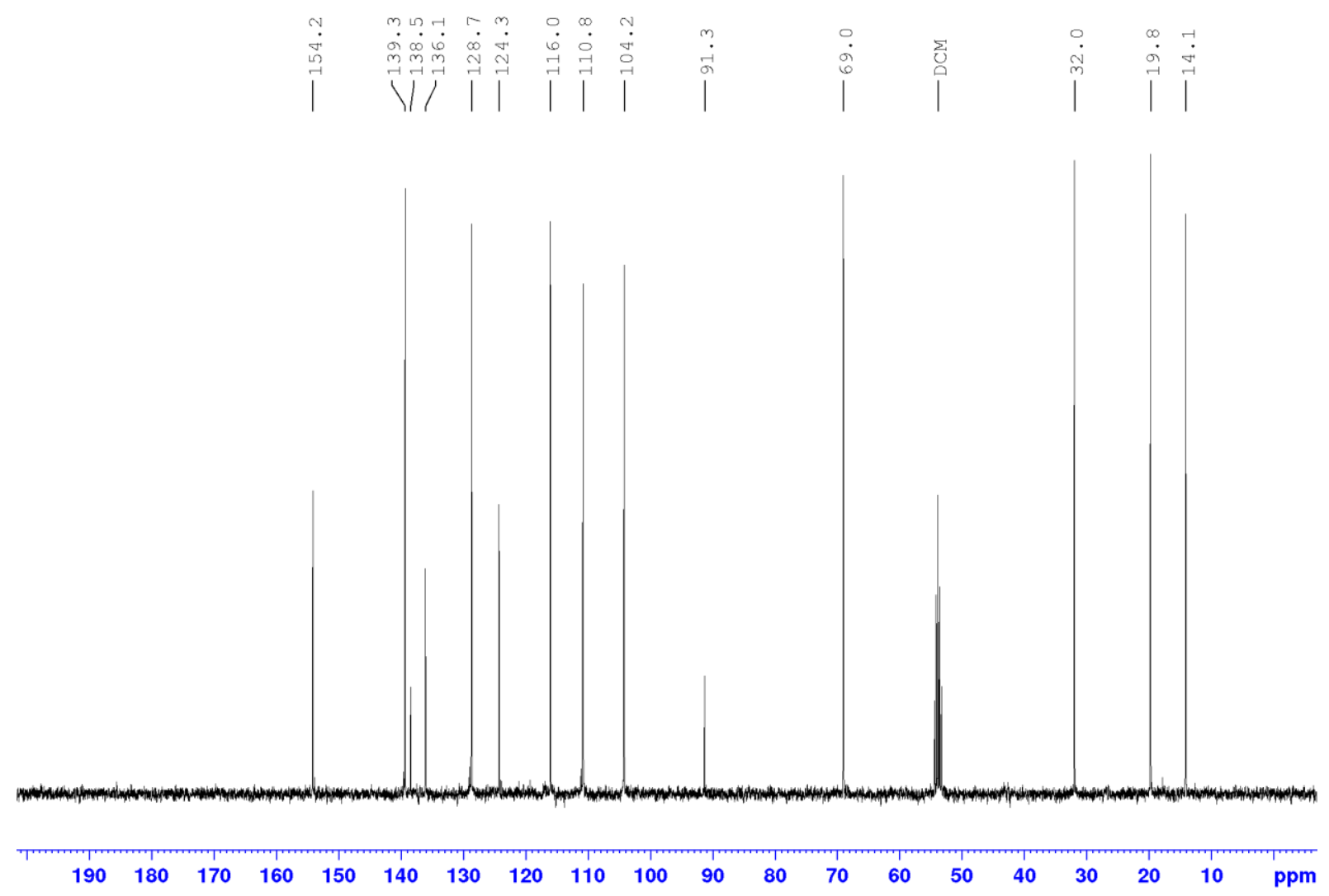

Figure S2. ${ }^{13} \mathrm{C}\left\{{ }^{1} \mathrm{H}\right\} \mathrm{NMR}$ of $\mathbf{2} \mathbf{b}$ in $\mathrm{CD}_{2} \mathrm{Cl}_{2}$ at a $100.6 \mathrm{MHz}$ spectrometer at $298 \mathrm{~K}$.

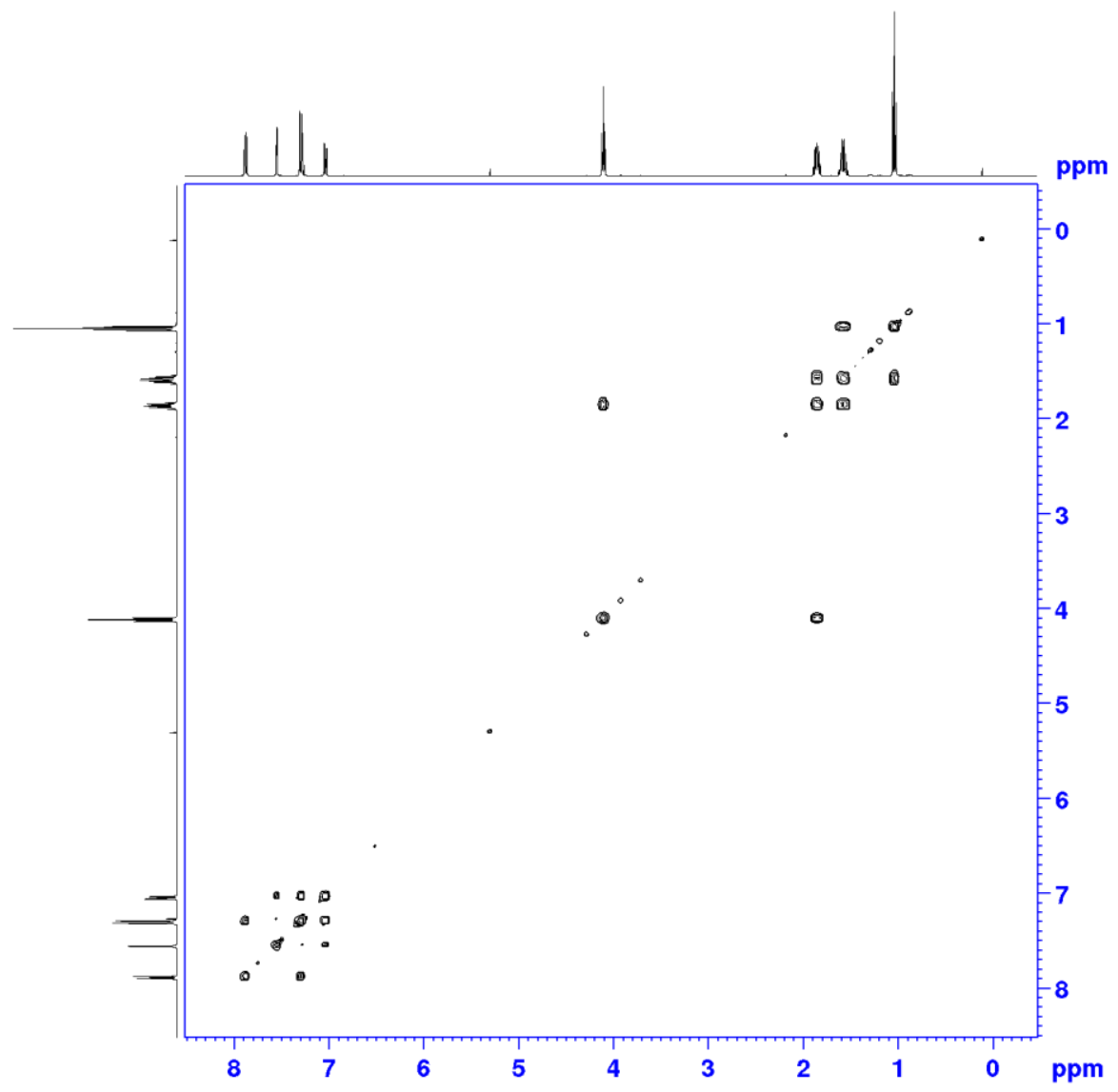

Figure S3. ${ }^{1} \mathrm{H},{ }^{1} \mathrm{H}$ gCOSY NMR spectrum of $\mathbf{2} \mathbf{b}$ in $\mathrm{CDCl}_{3}$ at a $400 \mathrm{MHz}$ spectrometer at $298 \mathrm{~K}$. 


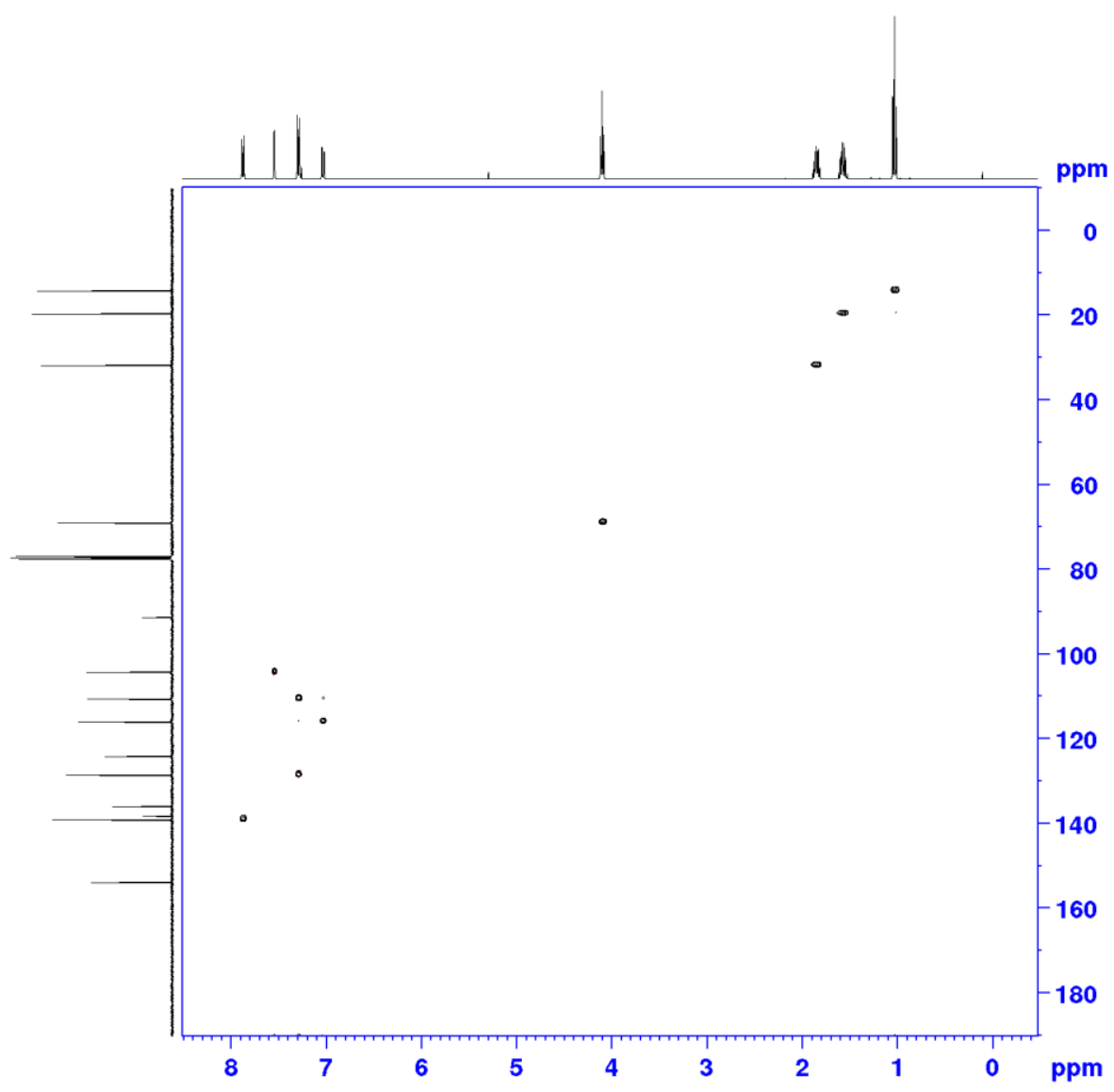

Figure S4. ${ }^{1} \mathrm{H},{ }^{13} \mathrm{C}$ gHSQC NMR spectrum of $\mathbf{2} \mathbf{b}$ in $\mathrm{CDCl}_{3}$ at a $400 \mathrm{MHz}$ spectrometer at $298 \mathrm{~K}$.

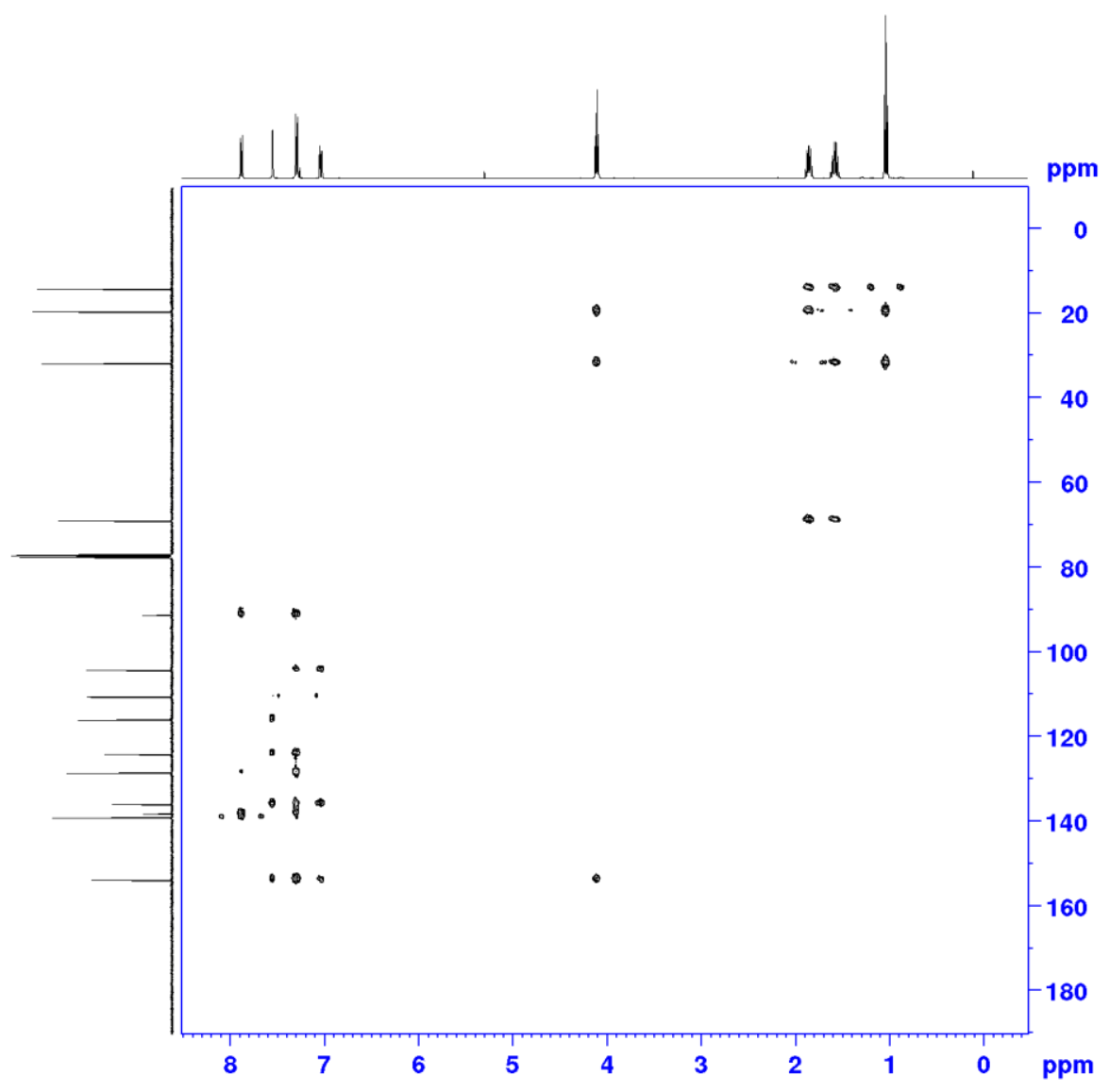

Figure S5. ${ }^{1} \mathrm{H},{ }^{13} \mathrm{C}$ gHMBC NMR spectrum of $\mathbf{2 b}$ in $\mathrm{CDCl}_{3}$ at a $400 \mathrm{MHz}$ spectrometer at $298 \mathrm{~K}$. 

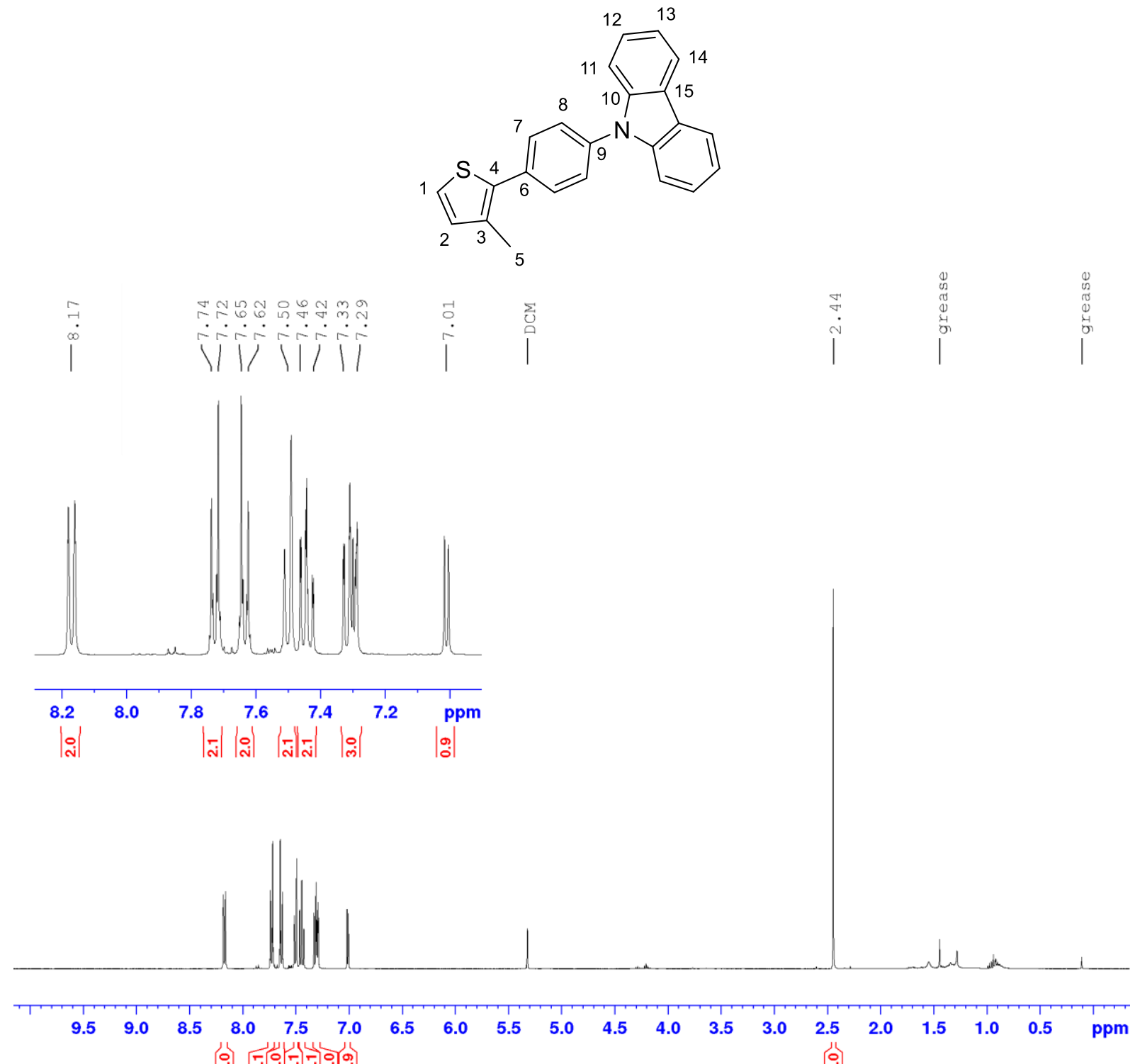

Figure S6. ${ }^{1} \mathrm{H}$ NMR of $\mathbf{4 a}$ in $\mathrm{CD}_{2} \mathrm{Cl}_{2}$ at a $400 \mathrm{MHz}$ spectrometer a $298 \mathrm{~K}$. 


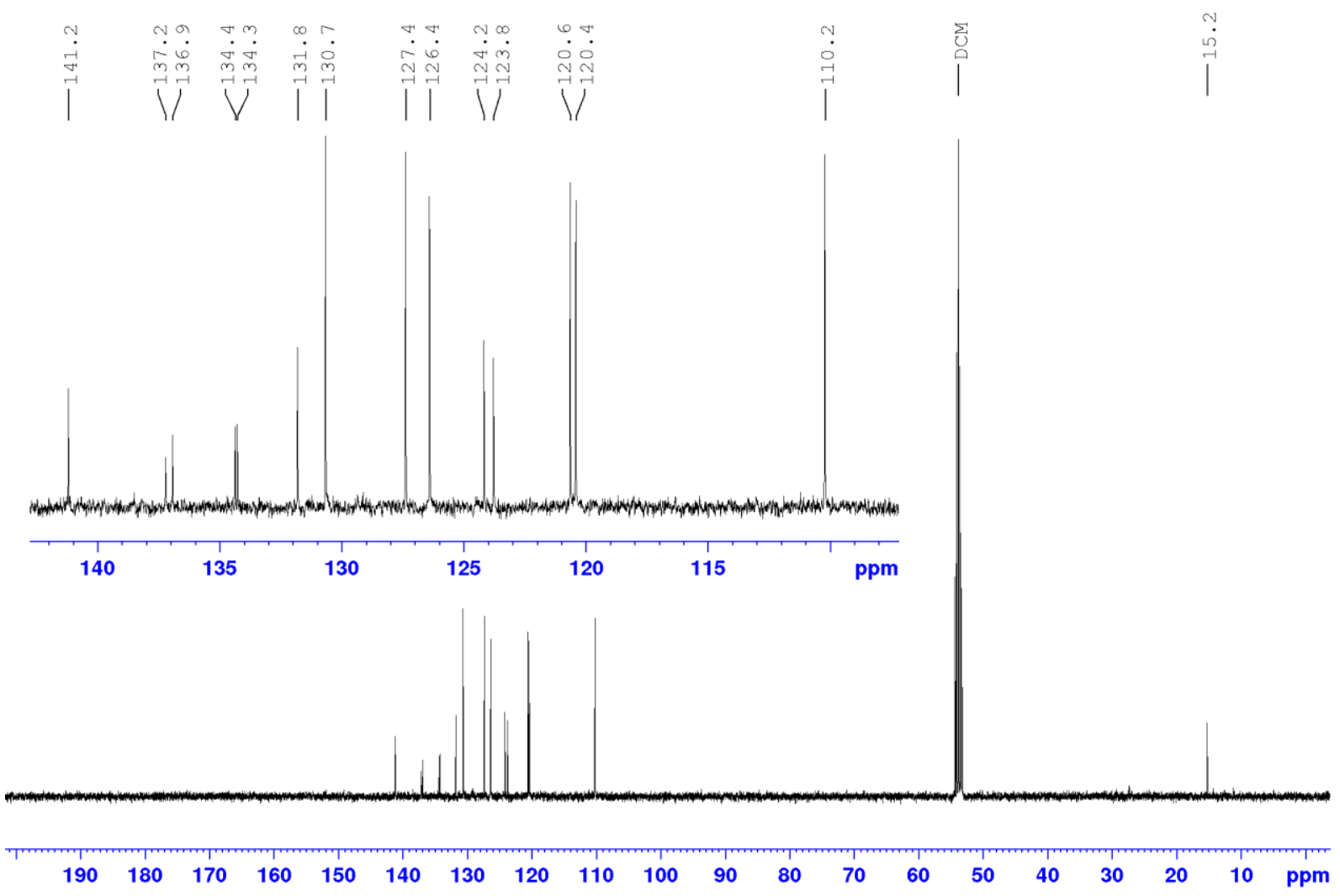

Figure S7. ${ }^{13} \mathrm{C}\left\{{ }^{1} \mathrm{H}\right\} \mathrm{NMR}$ of $\mathbf{4 a}$ in $\mathrm{CD}_{2} \mathrm{Cl}_{2}$ at a $100.6 \mathrm{MHz}$ spectrometer at $298 \mathrm{~K}$.

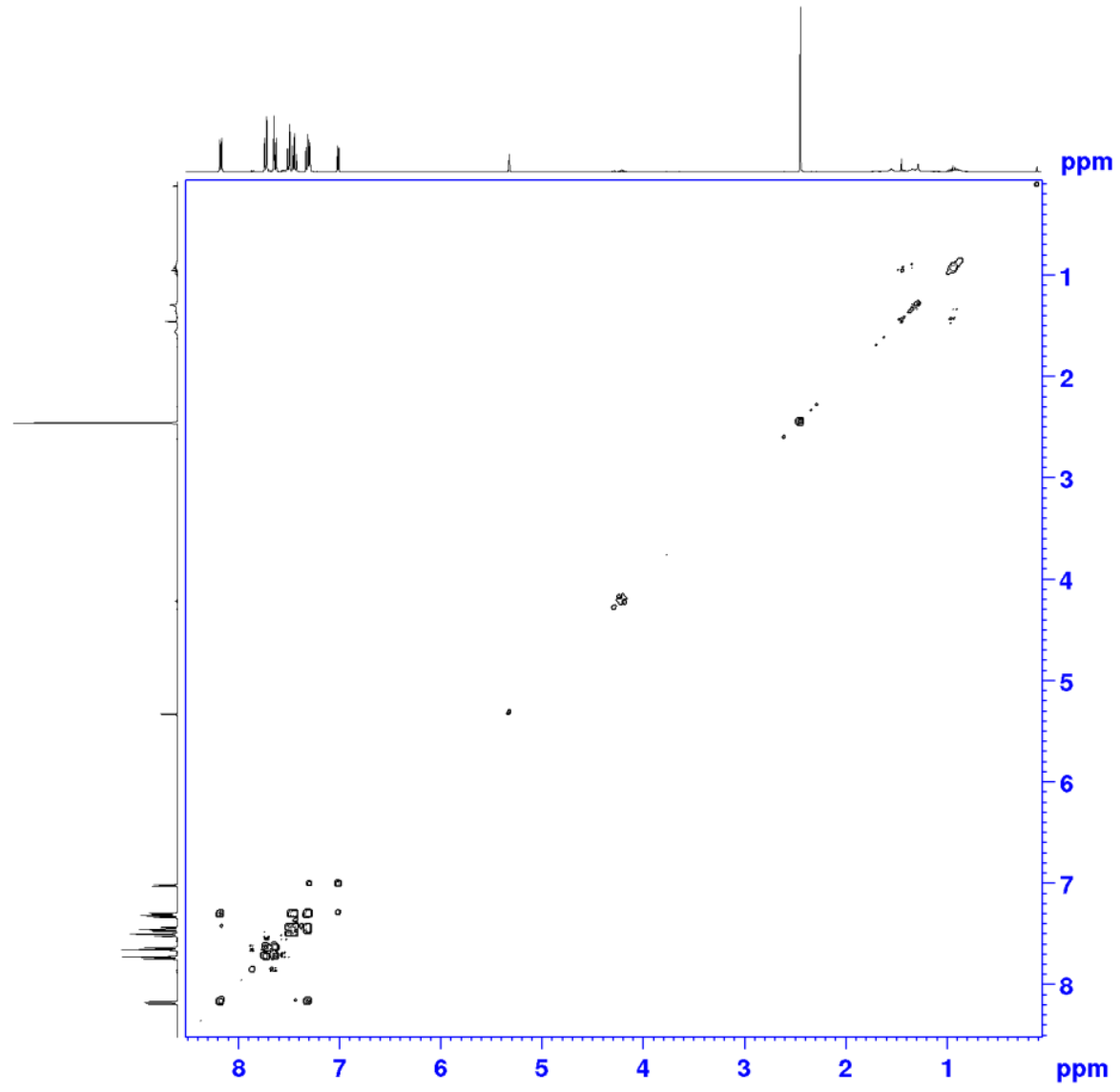

Figure S8. ${ }^{1} \mathrm{H},{ }^{1} \mathrm{H}$ gCOSY NMR spectrum of $4 \mathbf{a}$ in $\mathrm{CD}_{2} \mathrm{Cl}_{2}$ at a $400 \mathrm{MHz}$ spectrometer at $298 \mathrm{~K}$. 


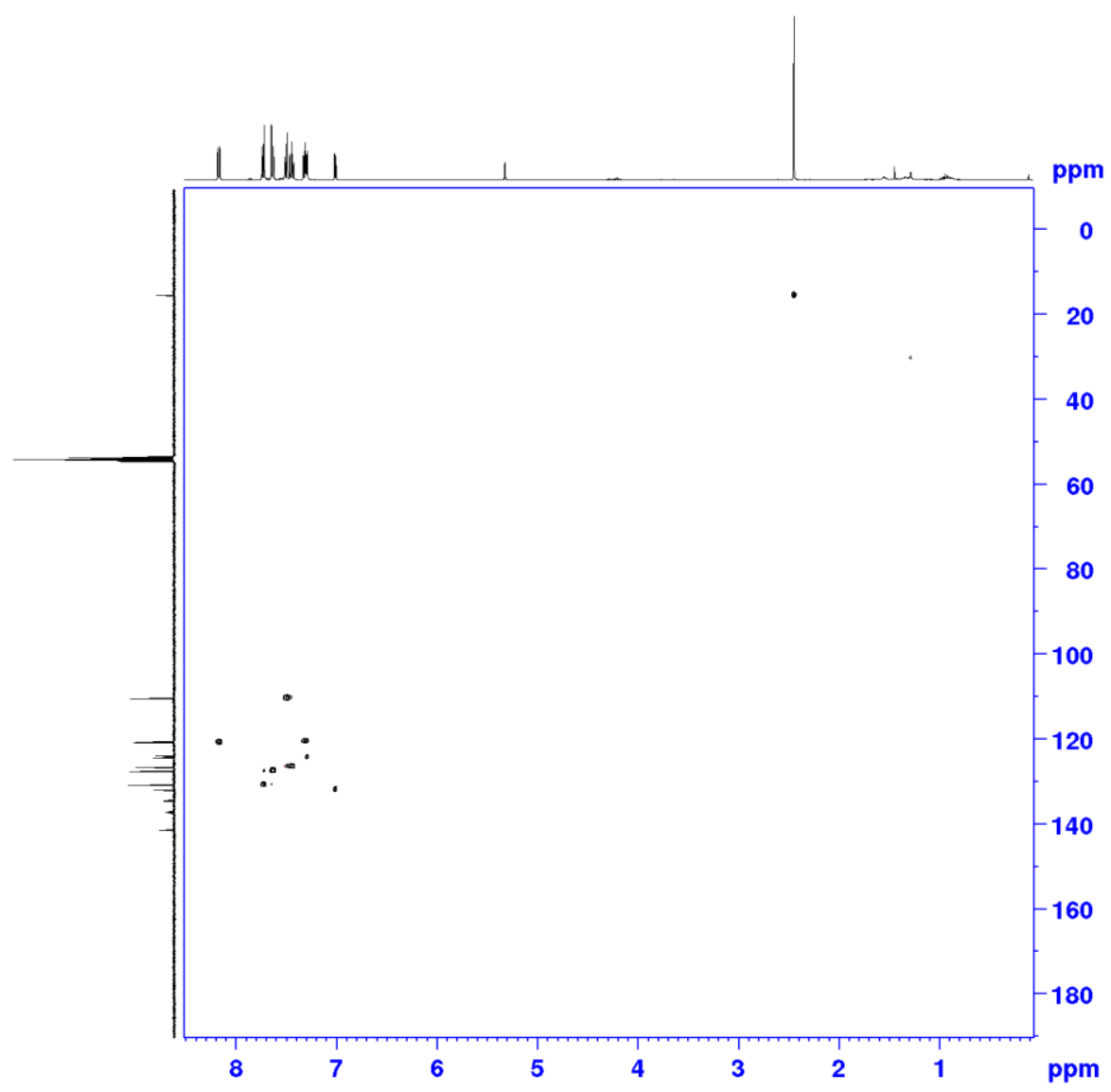

Figure S9. ${ }^{1} \mathrm{H},{ }^{13} \mathrm{C}$ gHSQC NMR spectrum of $4 \mathbf{a}$ in $\mathrm{CD}_{2} \mathrm{Cl}_{2}$ at a $400 \mathrm{MHz}$ spectrometer at $298 \mathrm{~K}$.

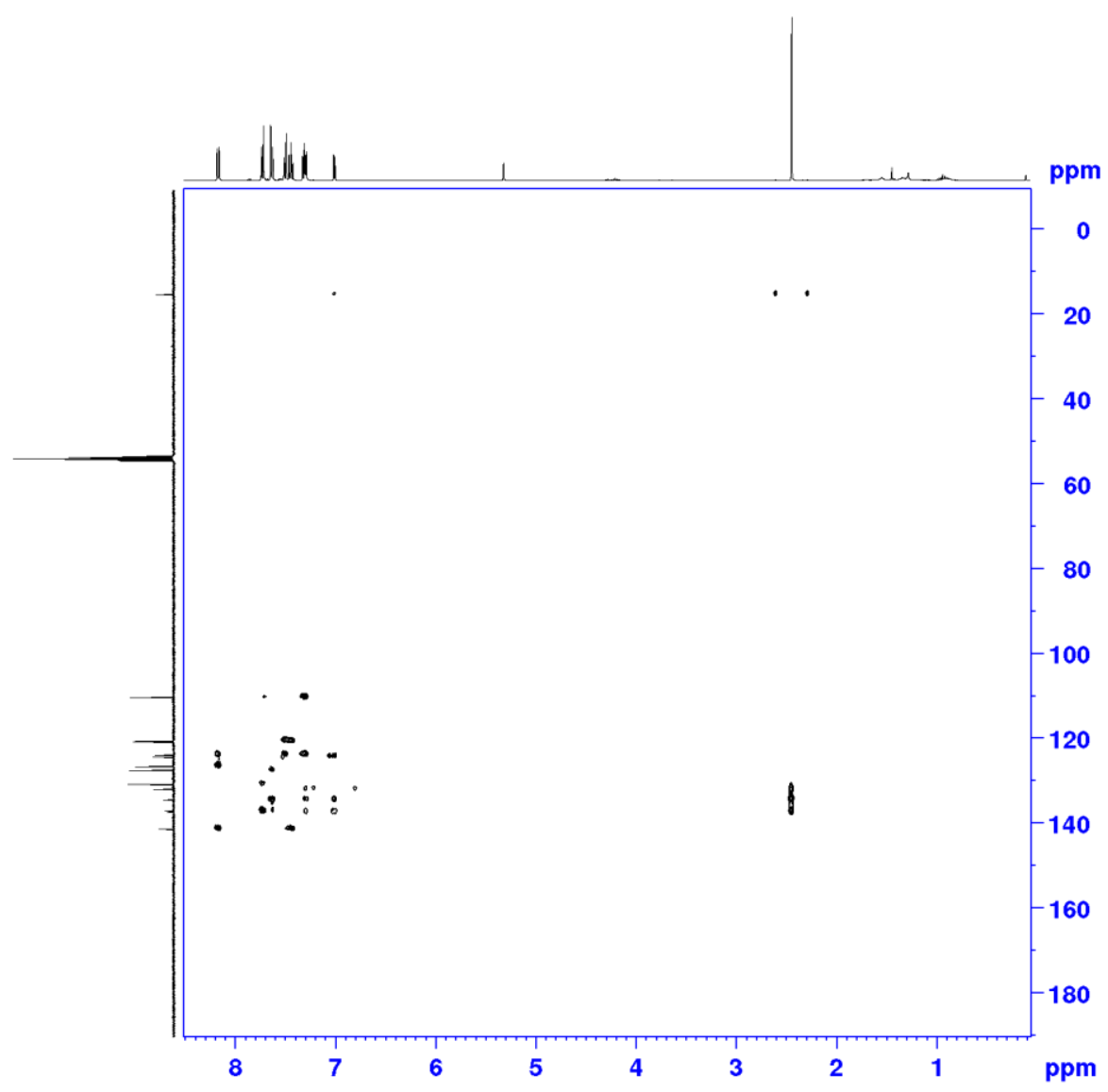

Figure S10. ${ }^{1} \mathrm{H},{ }^{13} \mathrm{C}$ gHMBC NMR spectrum of $4 \mathbf{a}$ in $\mathrm{CD}_{2} \mathrm{Cl}_{2}$ at a $400 \mathrm{MHz}$ spectrometer at $298 \mathrm{~K}$. 

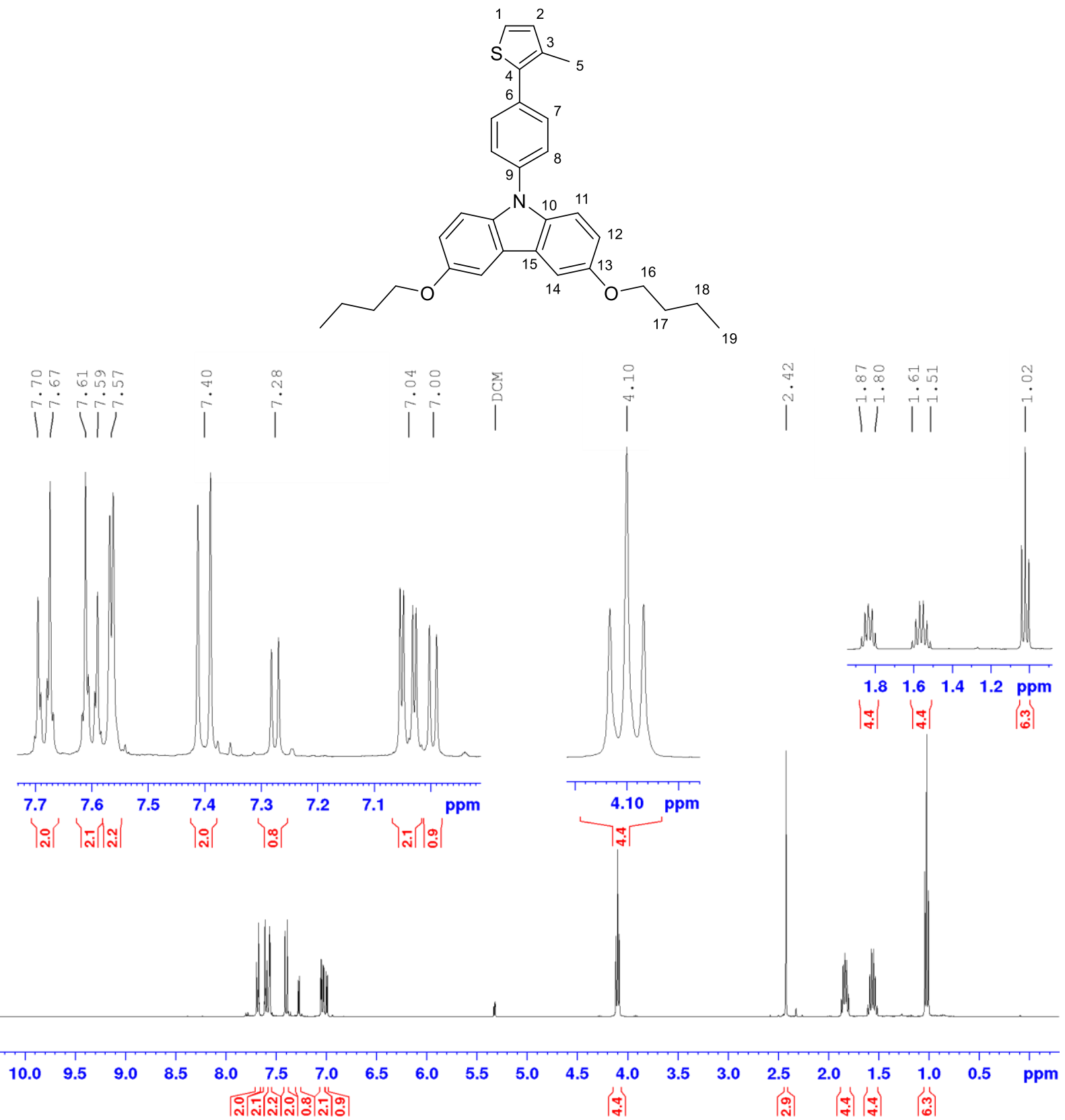

Figure S11. ${ }^{1} \mathrm{H}$ NMR of $\mathbf{4 b}$ in $\mathrm{CD}_{2} \mathrm{Cl}_{2}$ at a $400 \mathrm{MHz}$ spectrometer at $298 \mathrm{~K}$. 

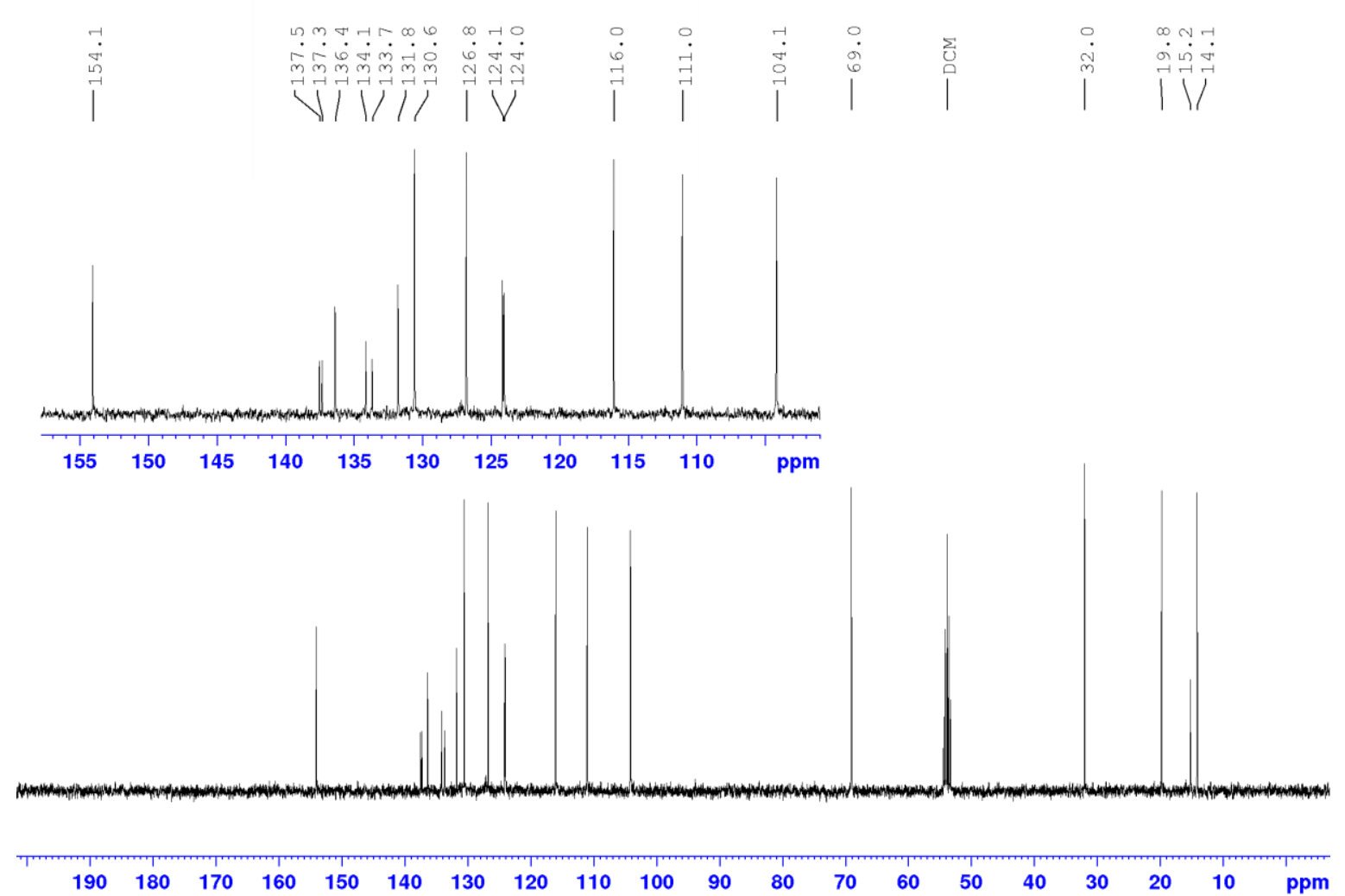

Figure S12. ${ }^{13} \mathrm{C}\left\{{ }^{1} \mathrm{H}\right\}$ NMR of $\mathbf{4 b}$ in $\mathrm{CD}_{2} \mathrm{Cl}_{2}$ at a $100.6 \mathrm{MHz}$ spectrometer at $298 \mathrm{~K}$.

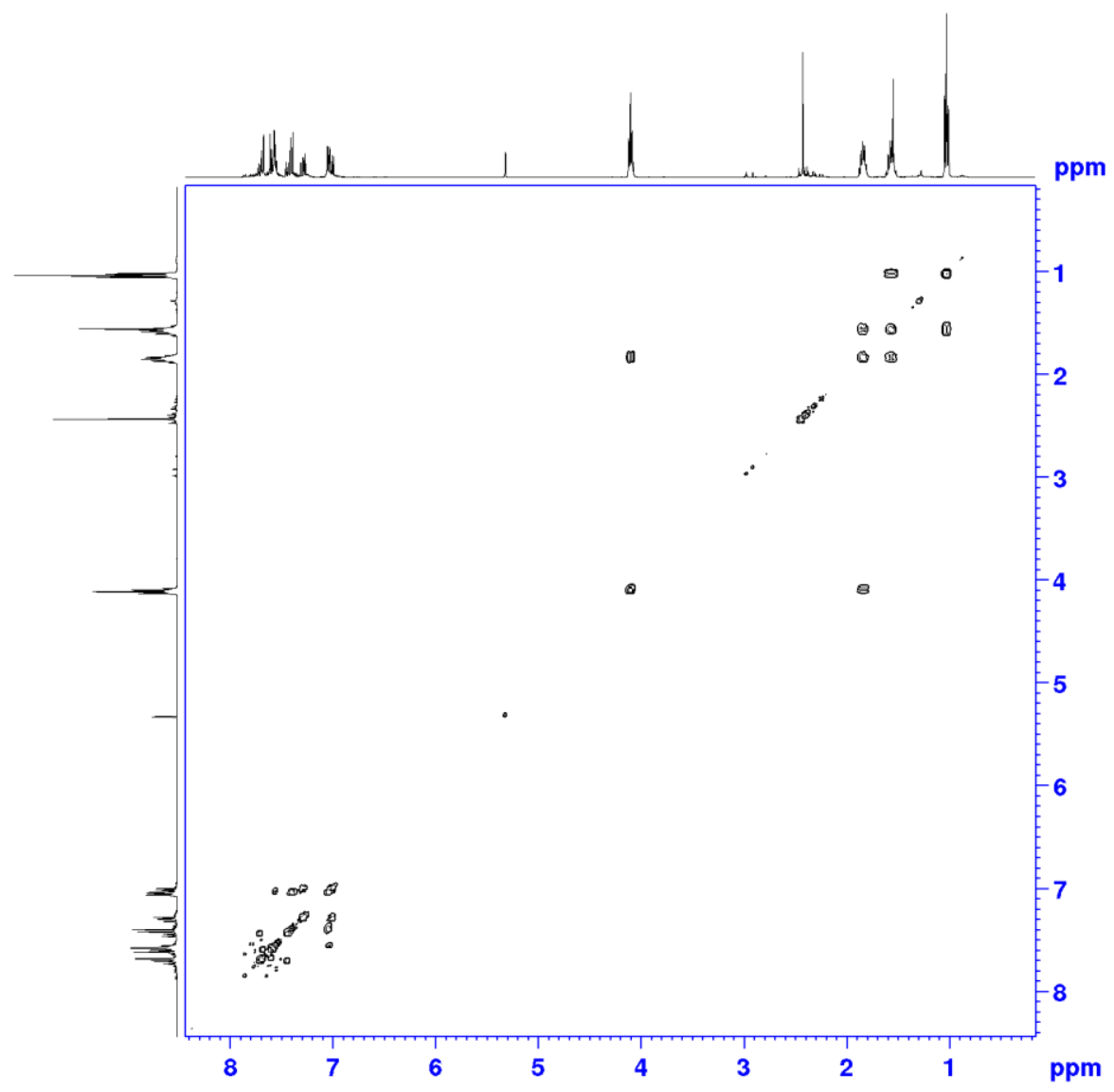

Figure S13. ${ }^{1} \mathrm{H},{ }^{1} \mathrm{H}$ gCOSY NMR spectrum of $\mathbf{4 b}$ in $\mathrm{CD}_{2} \mathrm{Cl}_{2}$ at a $400 \mathrm{MHz}$ spectrometer at $298 \mathrm{~K}$. 


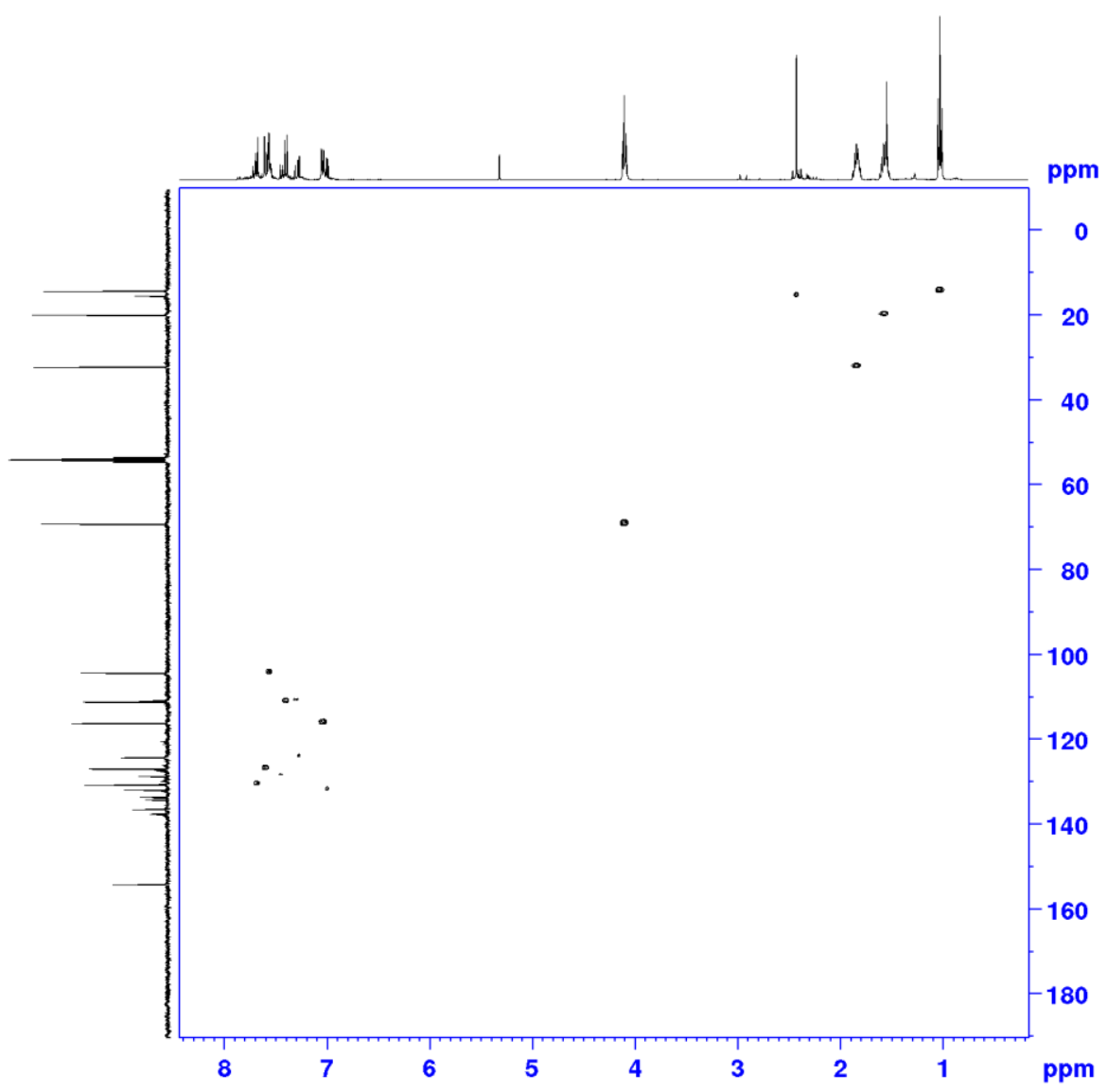

Figure S14. ${ }^{1} \mathrm{H},{ }^{13} \mathrm{C}$ gHSQC NMR spectrum of $\mathbf{4 b}$ in $\mathrm{CD}_{2} \mathrm{Cl}_{2}$ at a $400 \mathrm{MHz}$ spectrometer at $298 \mathrm{~K}$.

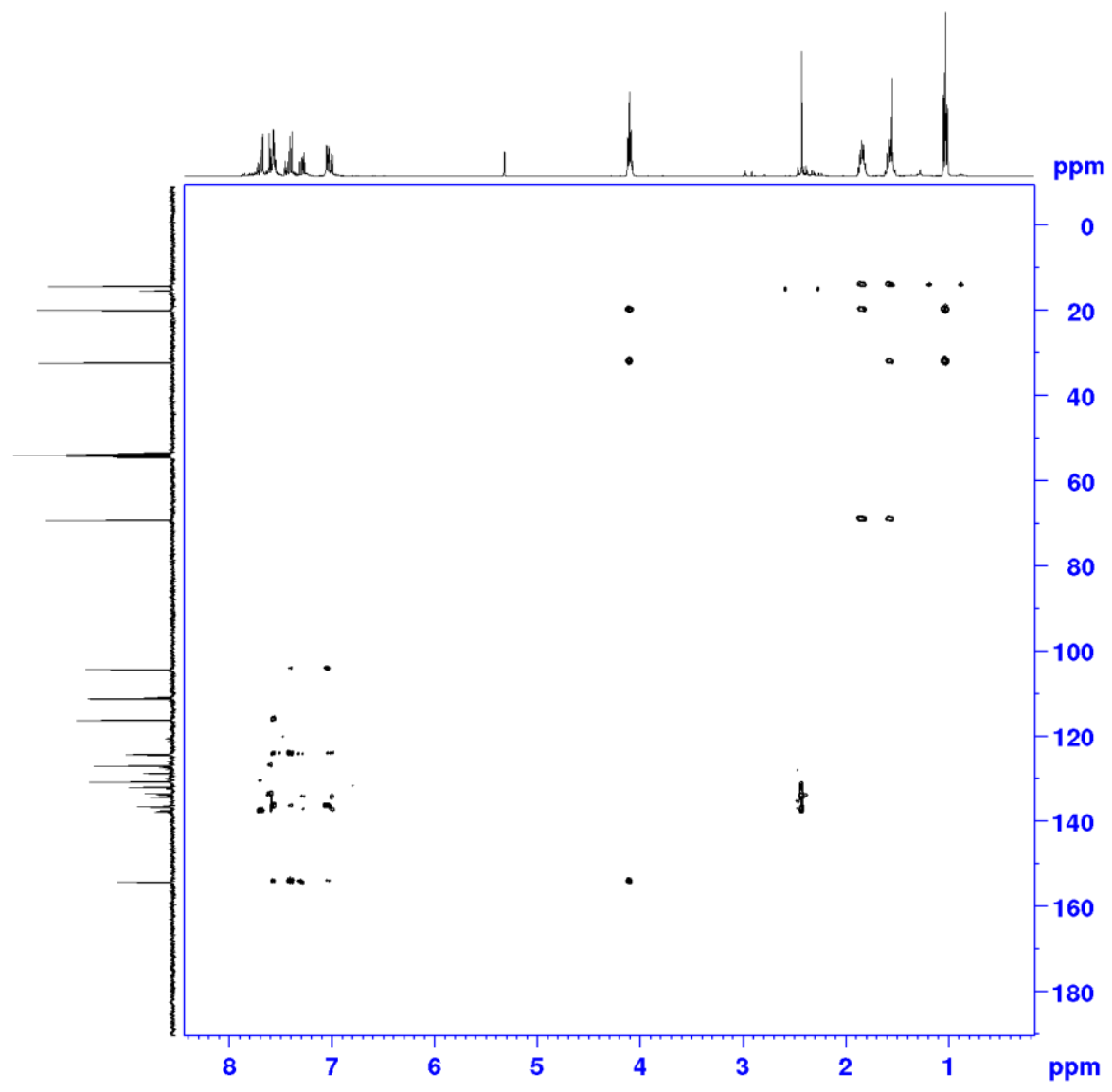

Figure S15. ${ }^{1} \mathrm{H},{ }^{13} \mathrm{C}$ gHMBC NMR spectrum of $\mathbf{4 b}$ in $\mathrm{CD}_{2} \mathrm{Cl}_{2}$ at a $400 \mathrm{MHz}$ spectrometer at $298 \mathrm{~K}$. 

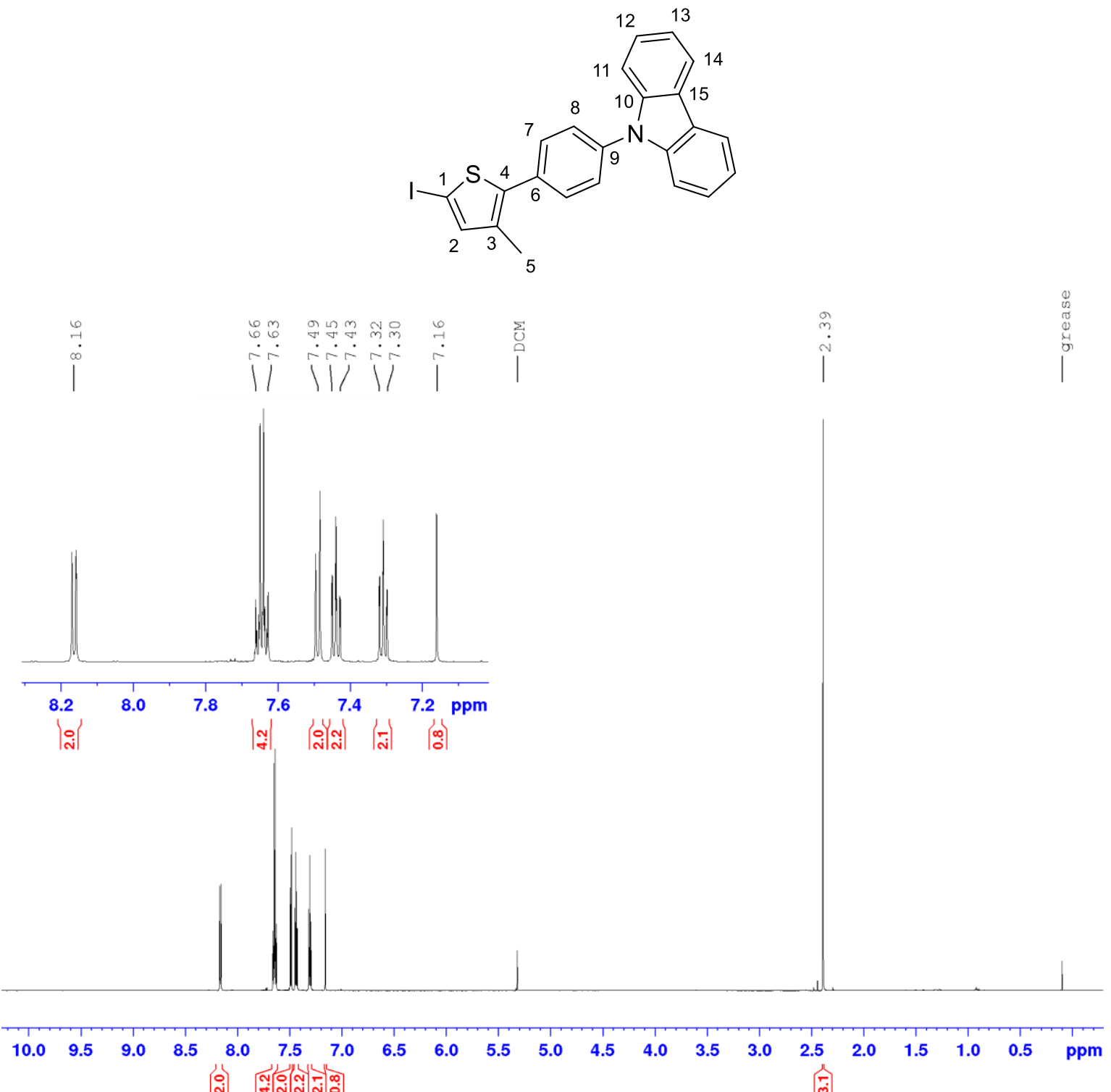

Figure S16. ${ }^{1} \mathrm{H}$ NMR of $5 \mathbf{a}$ in $\mathrm{CD}_{2} \mathrm{Cl}_{2}$ at a $400 \mathrm{MHz}$ spectrometer at $298 \mathrm{~K}$. 


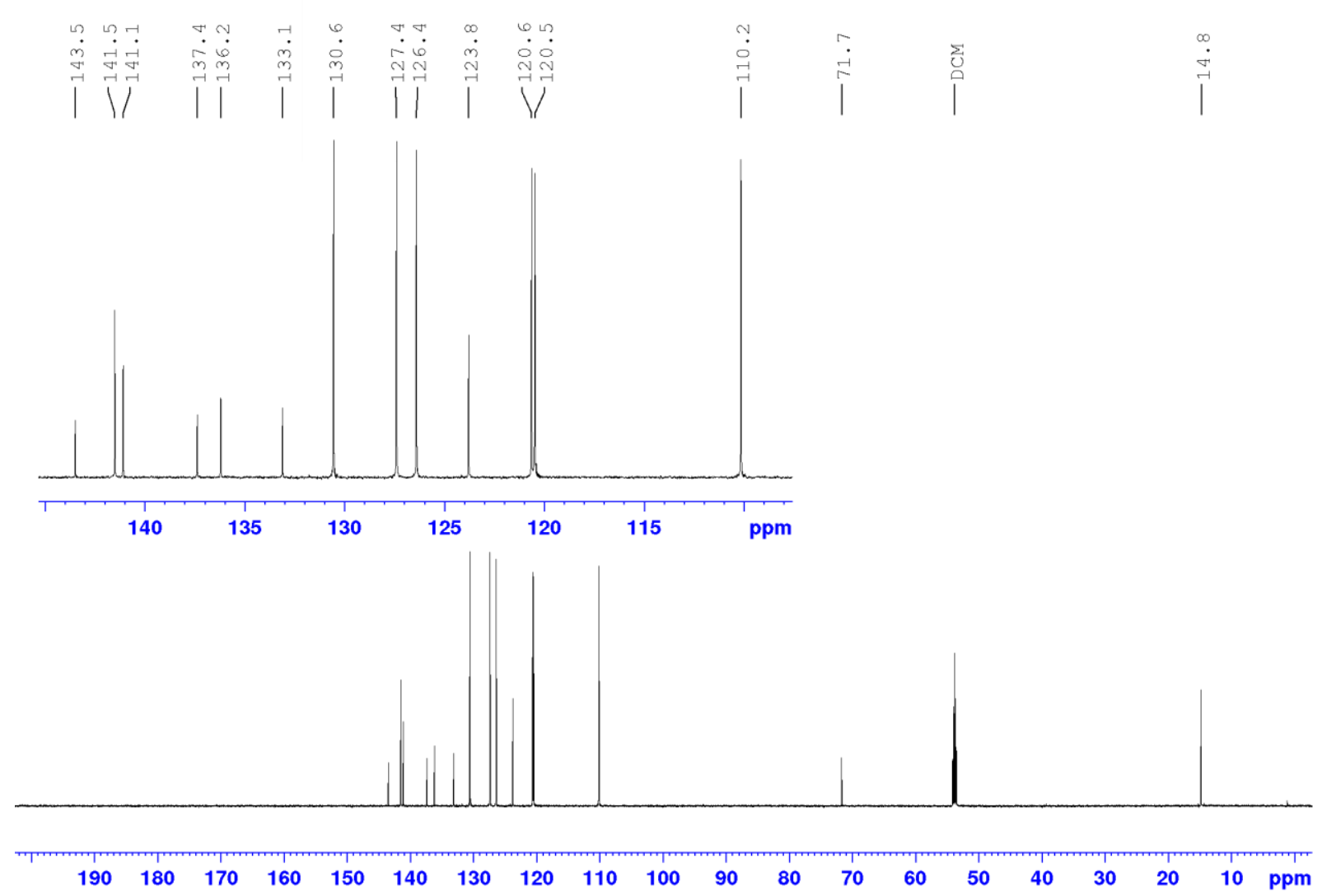

Figure S17. ${ }^{13} \mathrm{C}\left\{{ }^{1} \mathrm{H}\right\}$ NMR of $5 \mathbf{a}$ in $\mathrm{CD}_{2} \mathrm{Cl}_{2}$ at a $100.6 \mathrm{MHz}$ spectrometer at $298 \mathrm{~K}$.

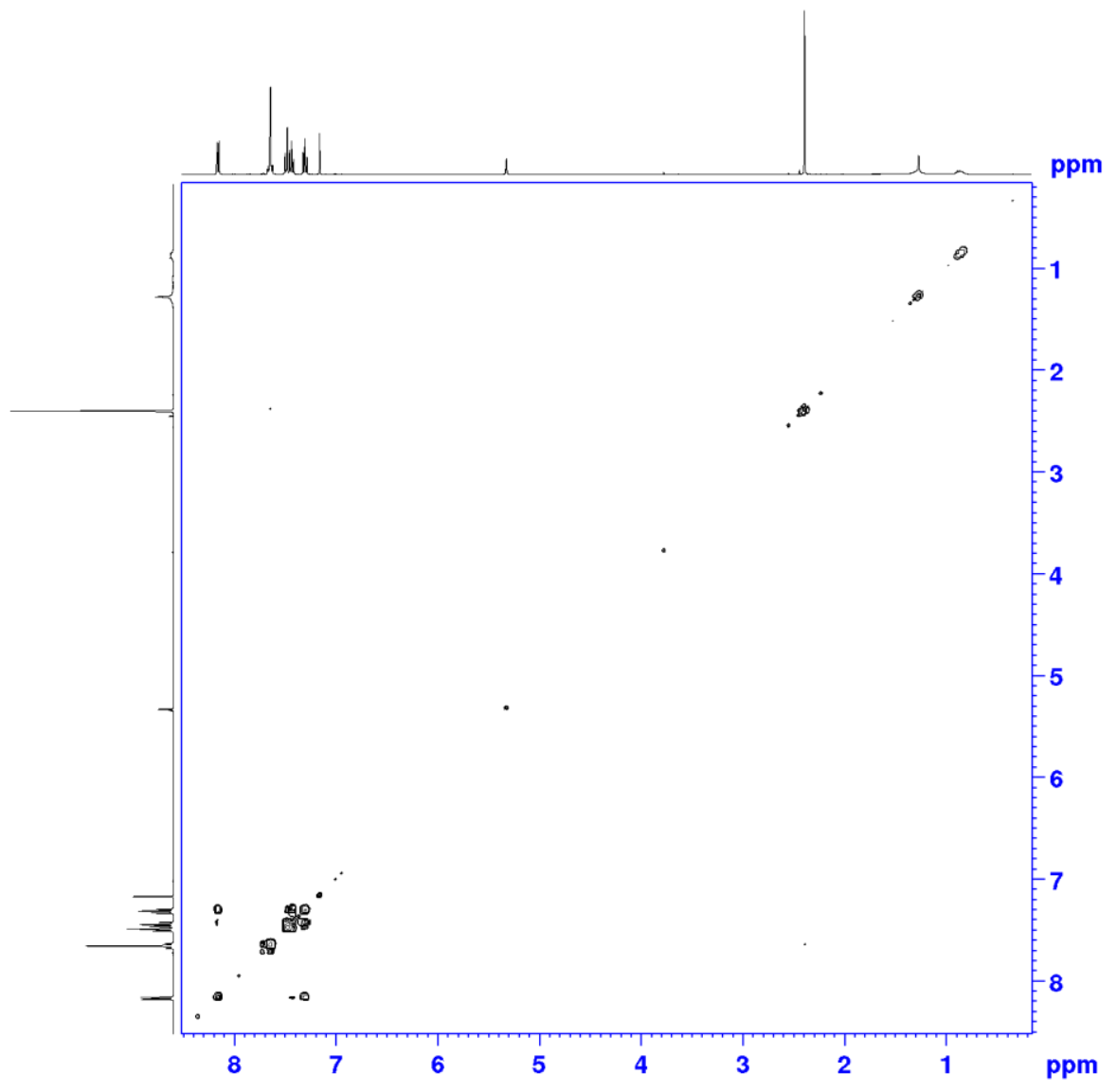

Figure S18. ${ }^{1} \mathrm{H},{ }^{1} \mathrm{H}$ gCOSY NMR spectrum of $\mathbf{5 a}$ in $\mathrm{CD}_{2} \mathrm{Cl}_{2}$ at a $400 \mathrm{MHz}$ spectrometer at $298 \mathrm{~K}$. 


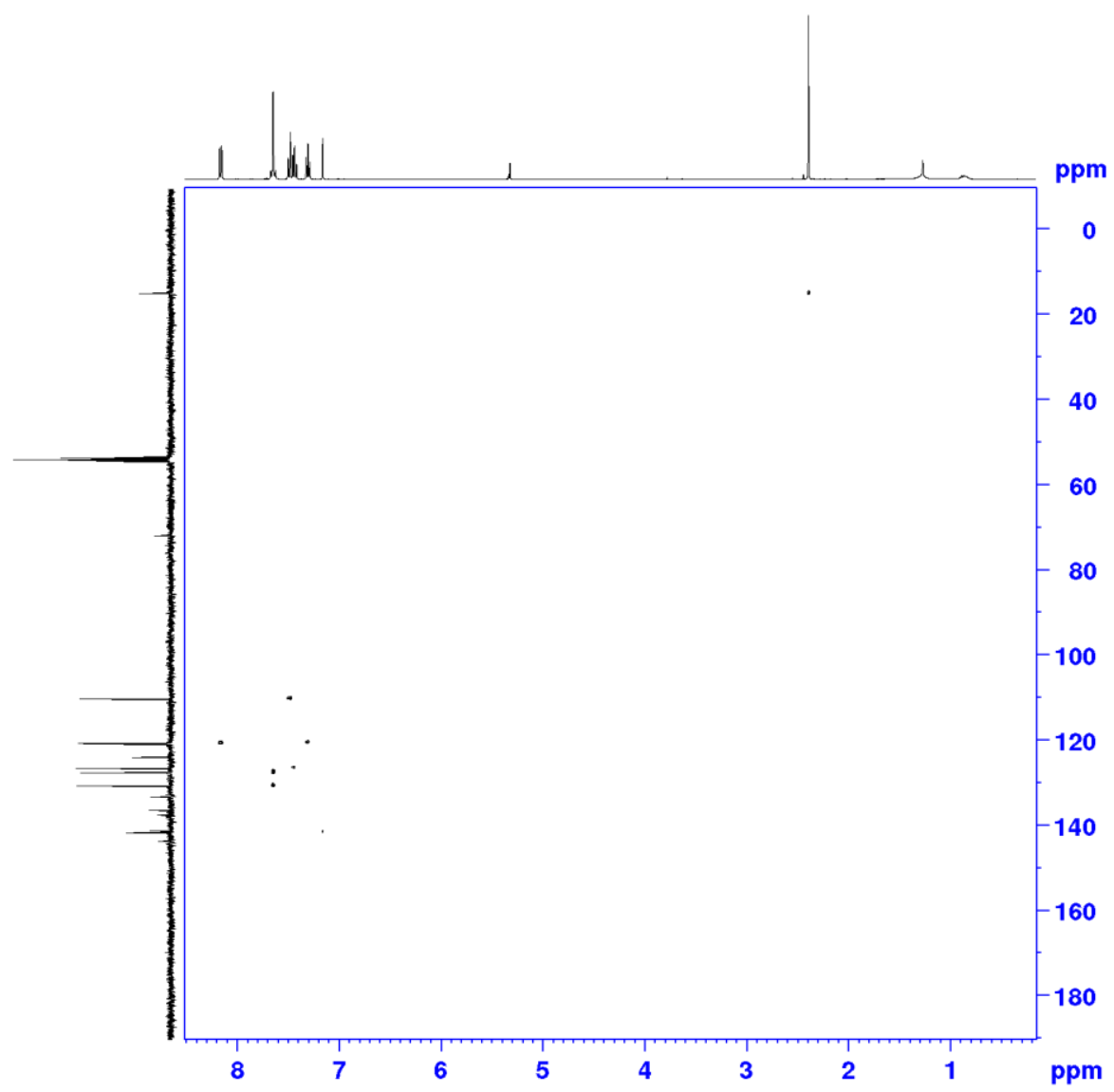

Figure S19. ${ }^{1} \mathrm{H},{ }^{13} \mathrm{C}$ gHSQC NMR spectrum of $\mathbf{5 a}$ in $\mathrm{CD}_{2} \mathrm{Cl}_{2}$ at a $400 \mathrm{MHz}$ spectrometer at $298 \mathrm{~K}$.

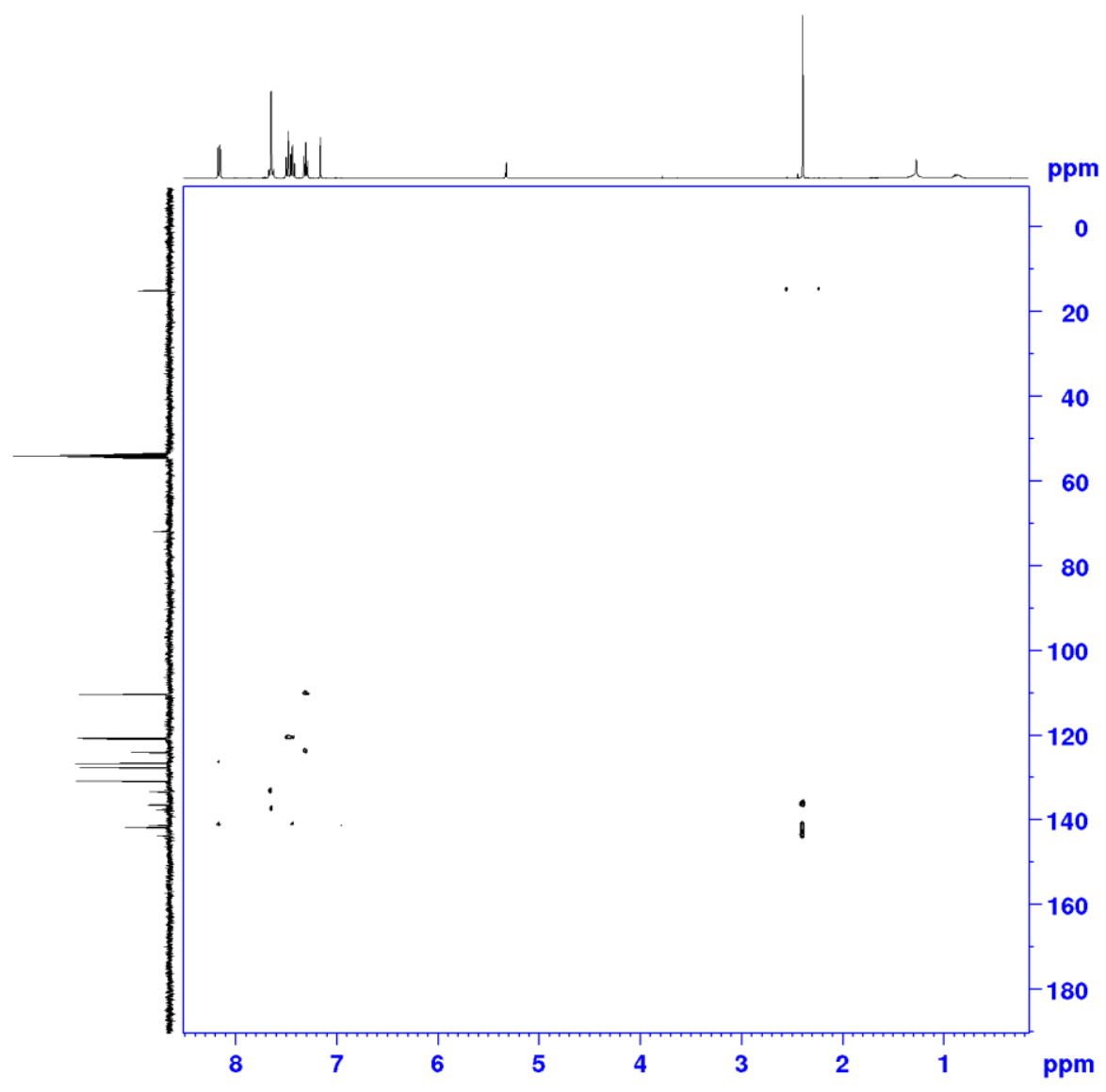

Figure S20: ${ }^{1} \mathrm{H},{ }^{13} \mathrm{C}$ gHMBC NMR spectrum of 5a in $\mathrm{CD}_{2} \mathrm{Cl}_{2}$ at a $400 \mathrm{MHz}$ spectrometer at $298 \mathrm{~K}$. 


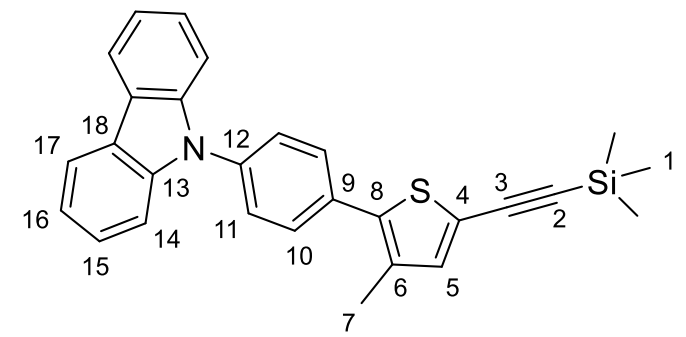

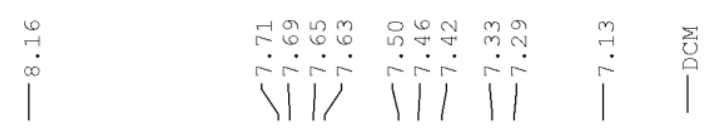
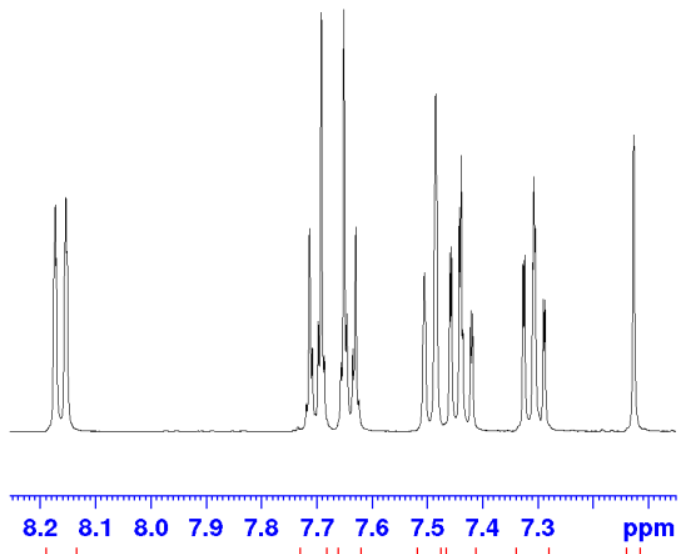

$\mid \circ \cdot$
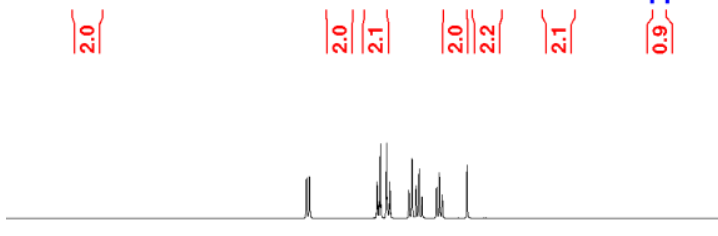

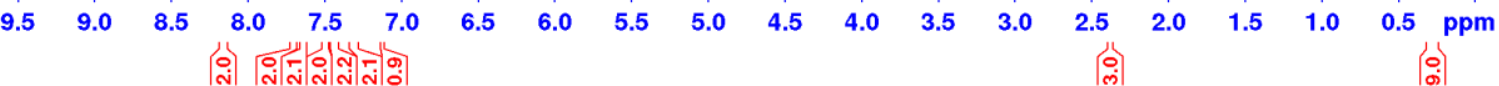

Figure S21. ${ }^{1} \mathrm{H}$ NMR of 6 in $\mathrm{CD}_{2} \mathrm{Cl}_{2}$ at a $400 \mathrm{MHz}$ spectrometer at $298 \mathrm{~K}$. 


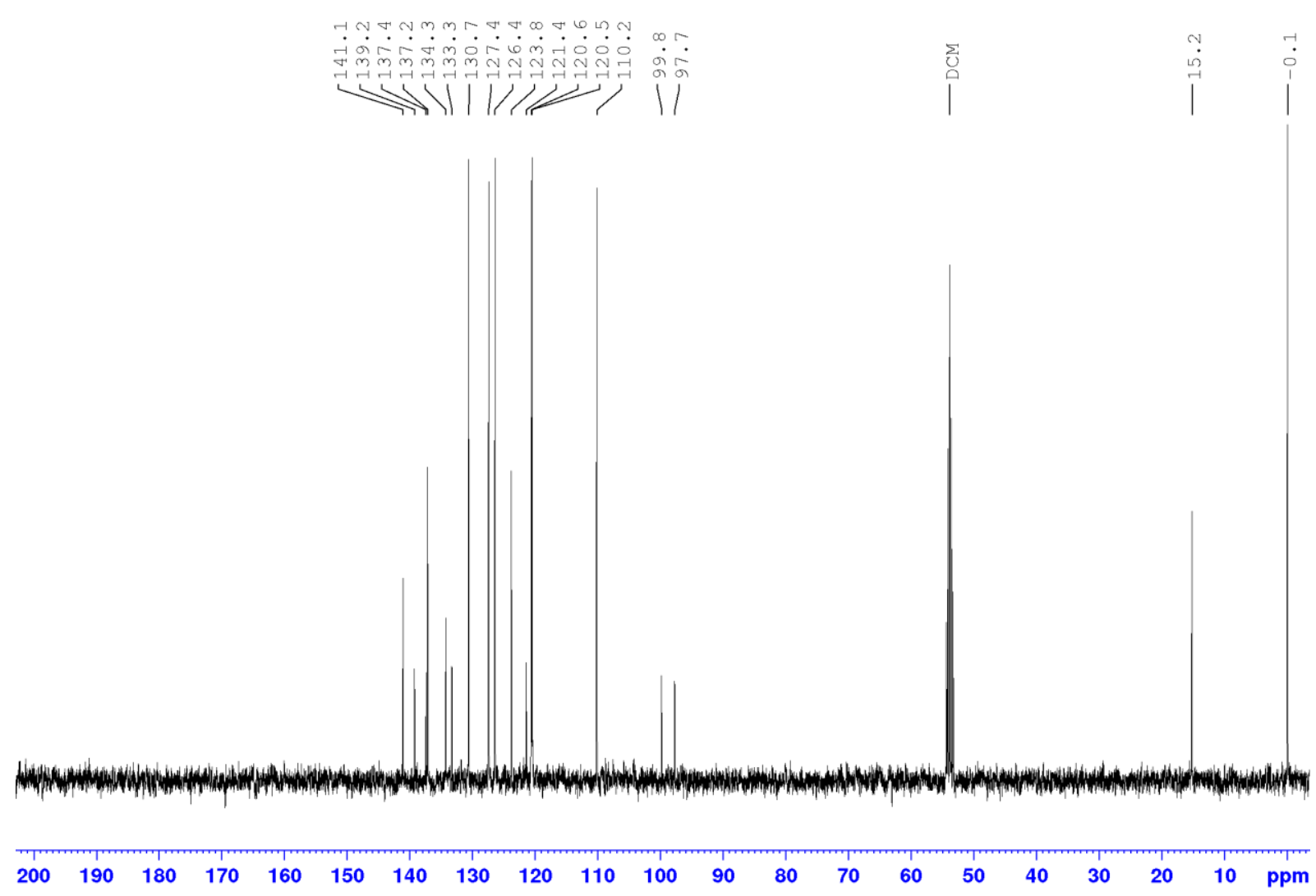

Figure S22. ${ }^{13} \mathrm{C}\left\{{ }^{1} \mathrm{H}\right\} \mathrm{NMR}$ of $\mathbf{6}$ in $\mathrm{CD}_{2} \mathrm{Cl}_{2}$ at a $100.6 \mathrm{MHz}$ spectrometer at $298 \mathrm{~K}$.

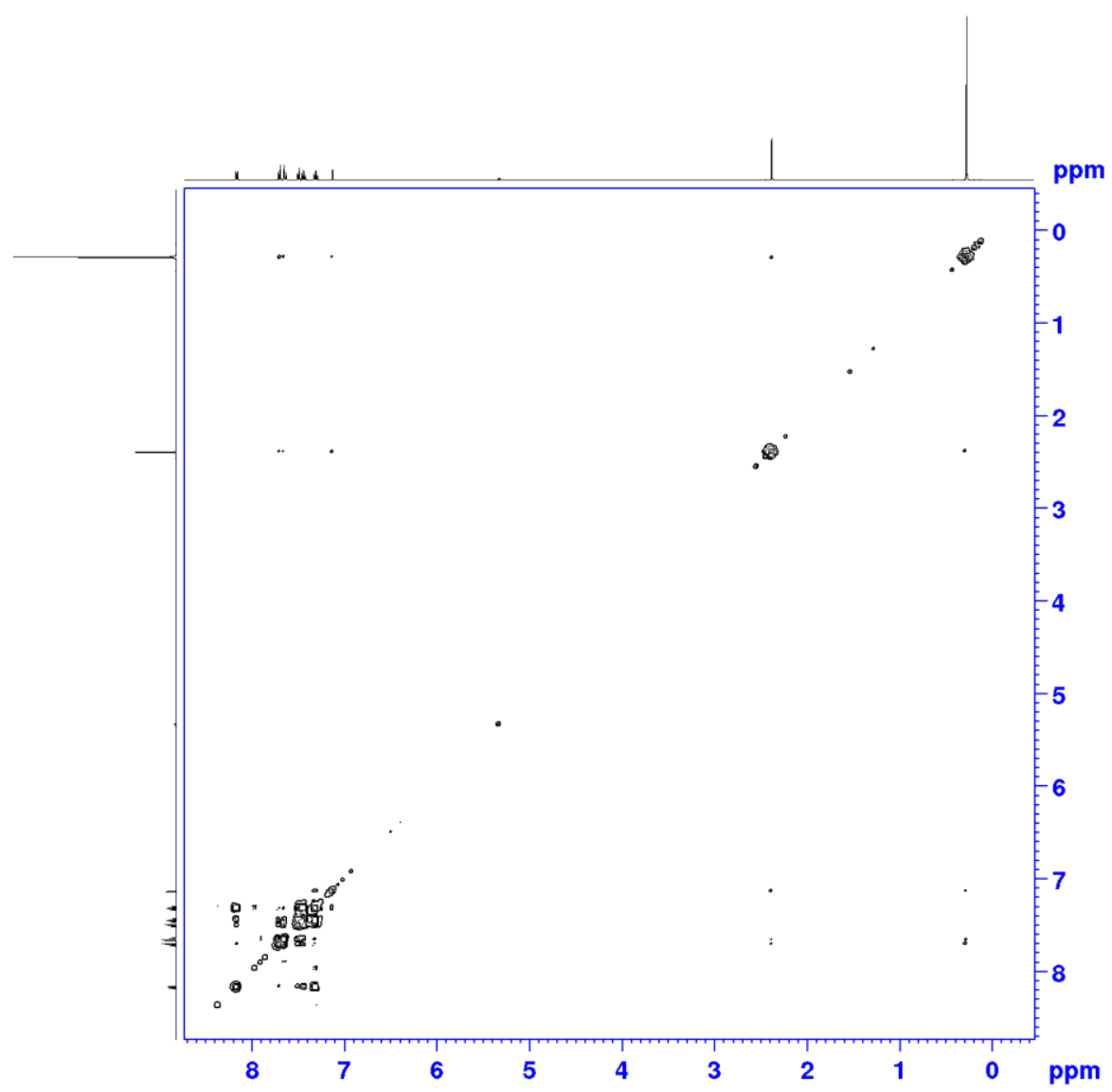

Figure S23. ${ }^{1} \mathrm{H},{ }^{1} \mathrm{H}$ gCOSY NMR spectrum of 6 in $\mathrm{CD}_{2} \mathrm{Cl}_{2}$ at a $400 \mathrm{MHz}$ spectrometer at $298 \mathrm{~K}$. 


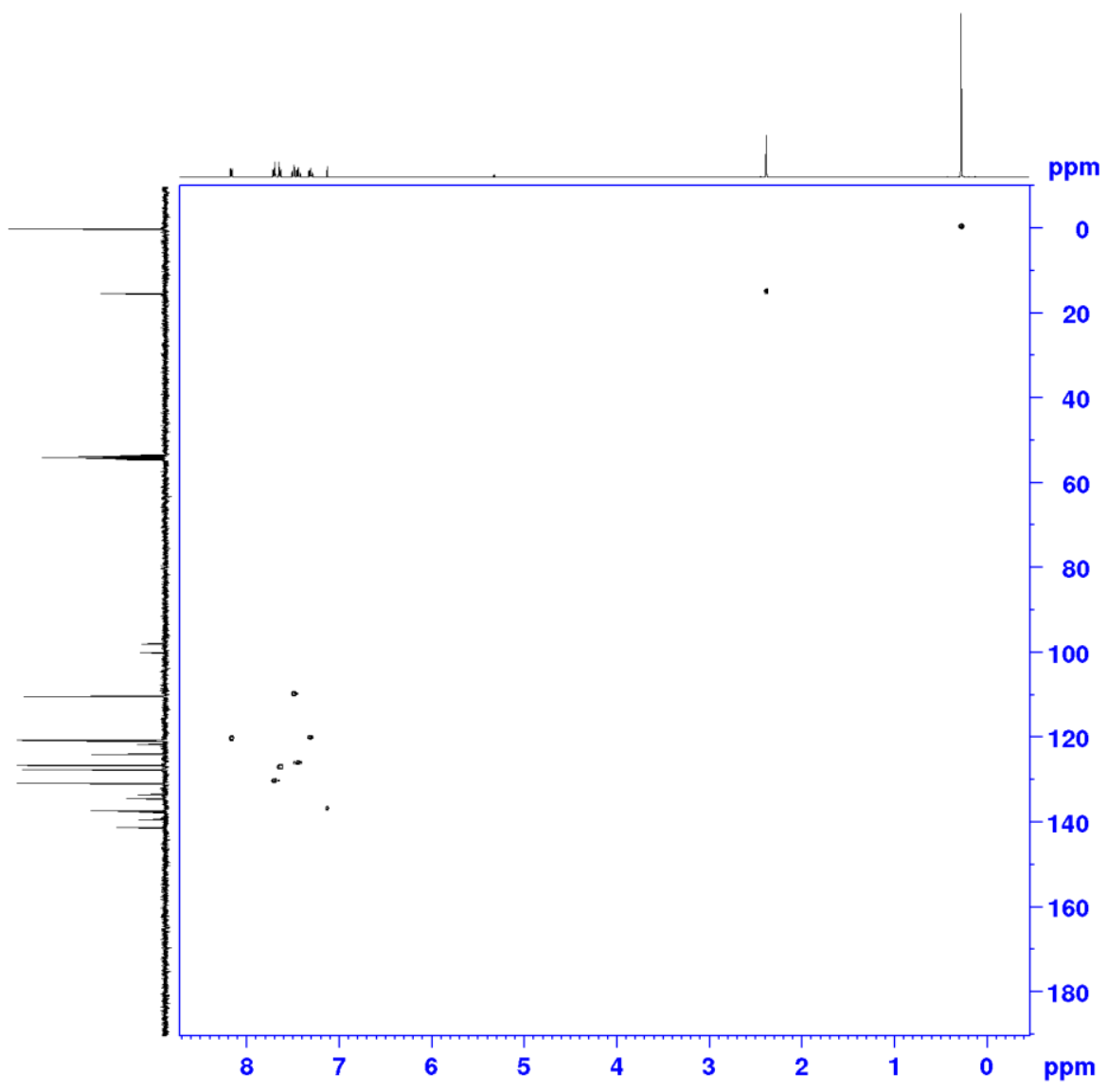

Figure S24. ${ }^{1} \mathrm{H},{ }^{13} \mathrm{C}$ gHSQC NMR spectrum of 6 in $\mathrm{CD}_{2} \mathrm{Cl}_{2}$ at a $400 \mathrm{MHz}$ spectrometer at $298 \mathrm{~K}$.

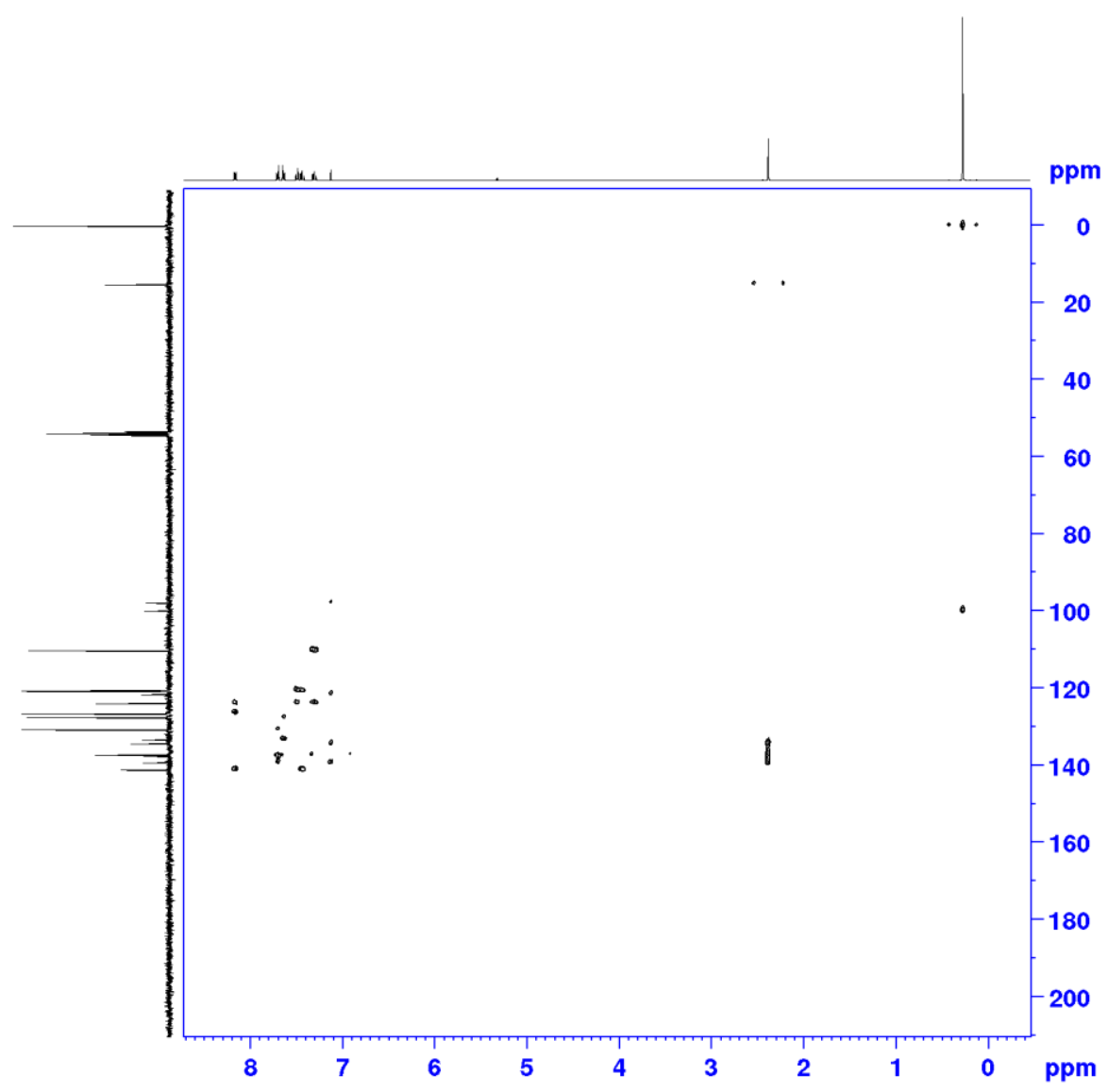

Figure S25. ${ }^{1} \mathrm{H},{ }^{13} \mathrm{C}$ gHMBC NMR spectrum of 6 in $\mathrm{CD}_{2} \mathrm{Cl}_{2}$ at a $400 \mathrm{MHz}$ spectrometer at $298 \mathrm{~K}$. 


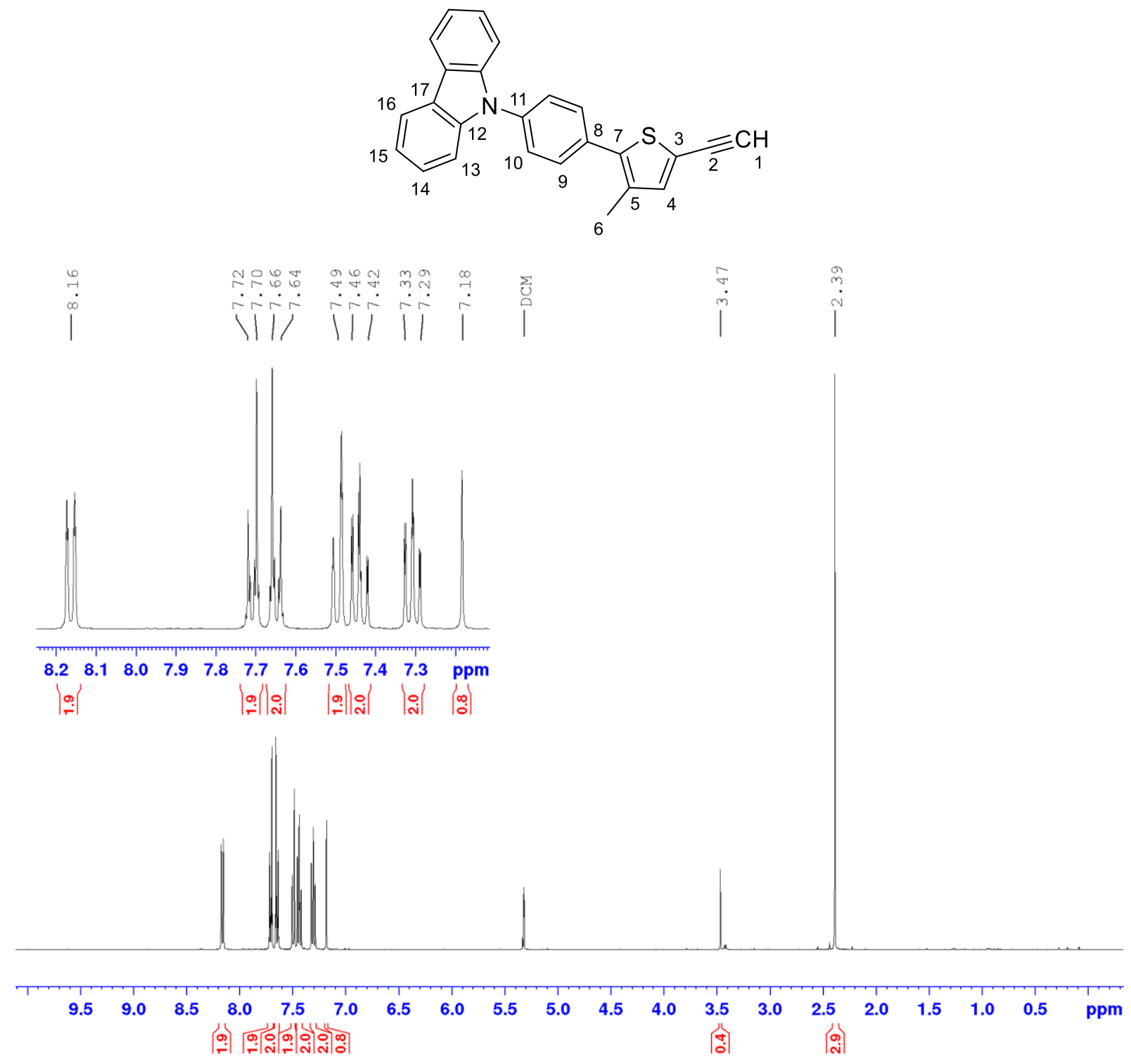

Figure S26. ${ }^{1} \mathrm{H}$ NMR of 7 in $\mathrm{CD}_{2} \mathrm{Cl}_{2}$ at a $400 \mathrm{MHz}$ spectrometer at $298 \mathrm{~K}$. 


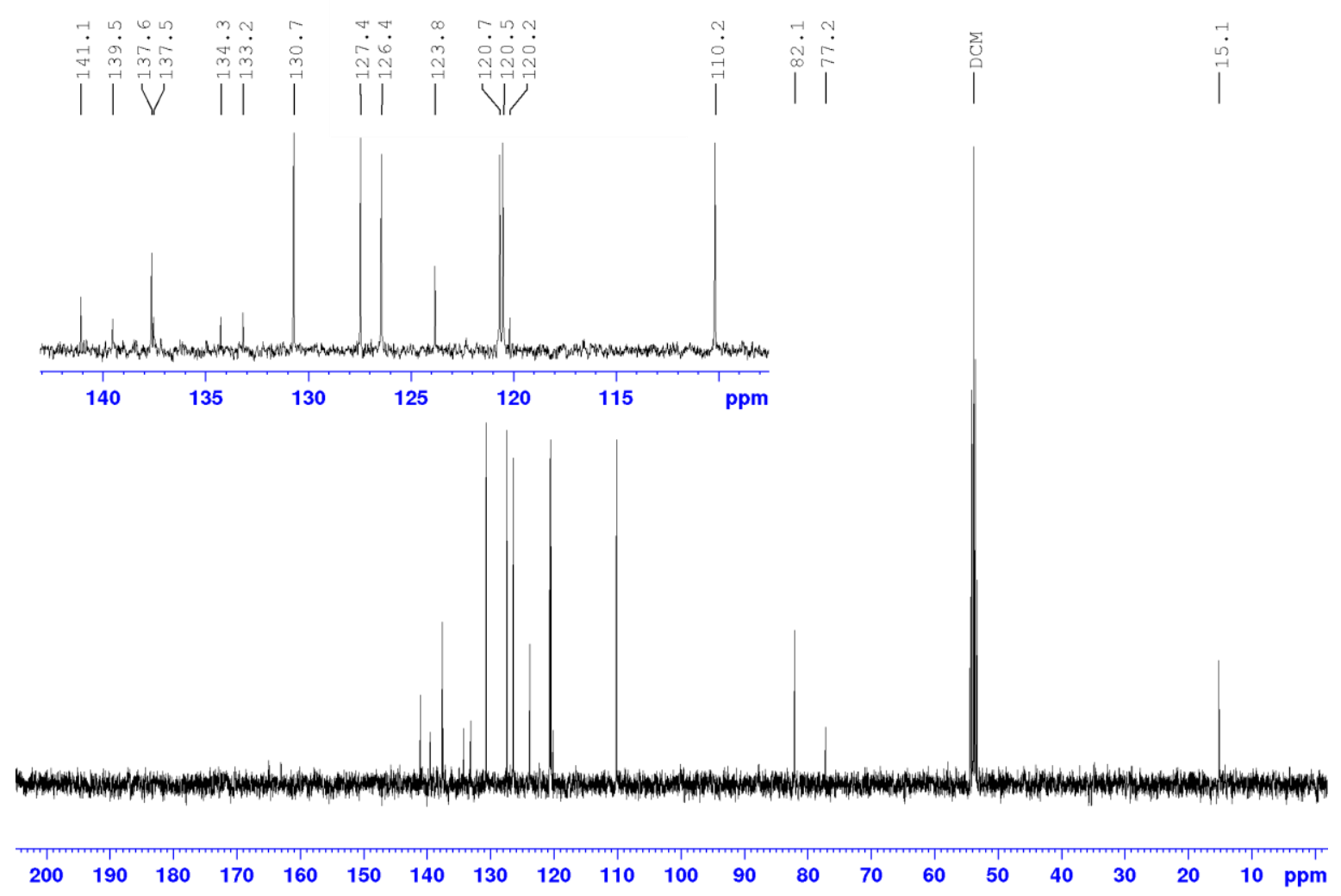

Figure S27. ${ }^{13} \mathrm{C}\left\{{ }^{1} \mathrm{H}\right\}$ NMR of 7 in $\mathrm{CD}_{2} \mathrm{Cl}_{2}$ at a $100.6 \mathrm{MHz}$ spectrometer at $298 \mathrm{~K}$.

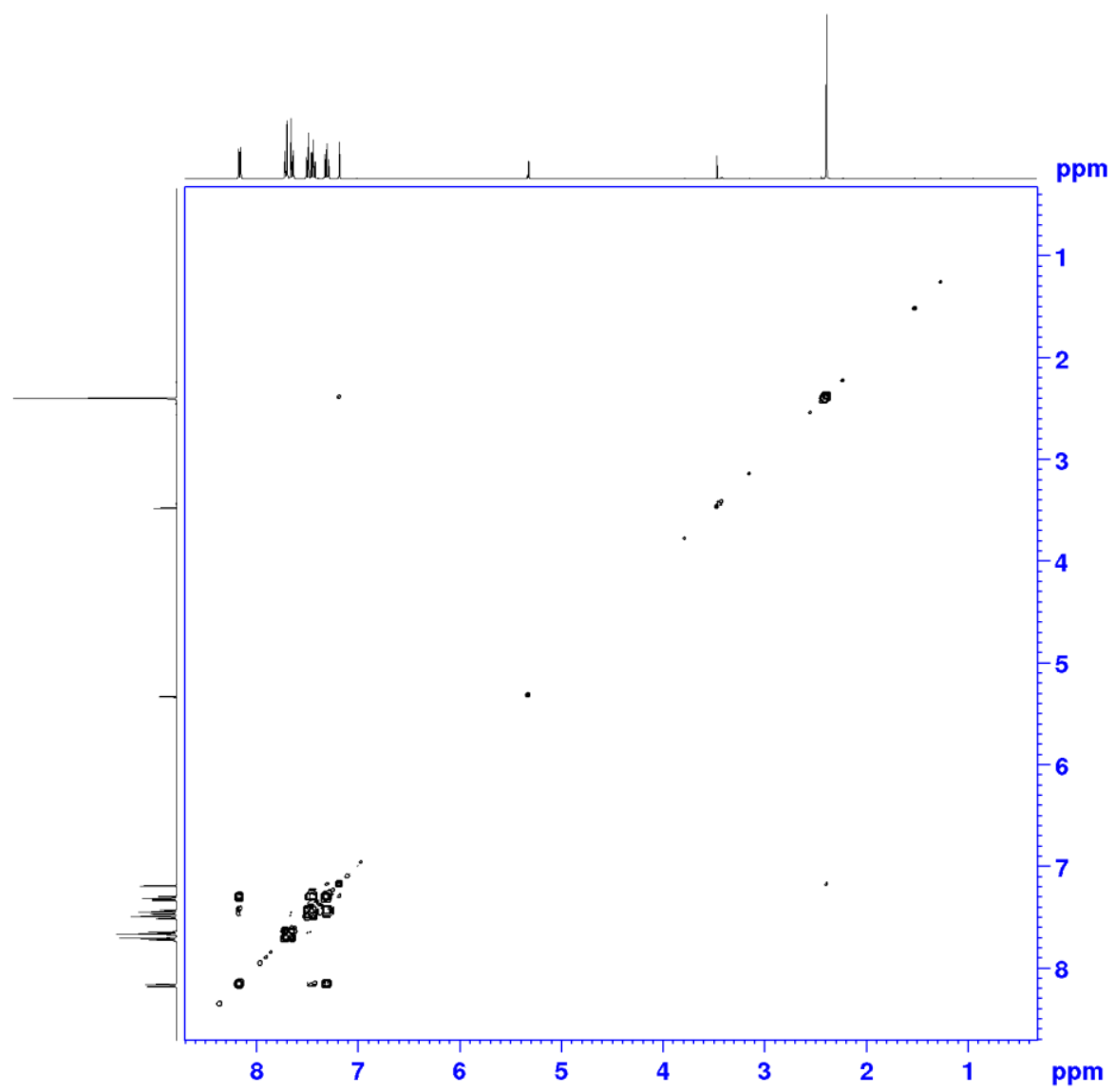

Figure S28. ${ }^{1} \mathrm{H},{ }^{1} \mathrm{H}$ gCOSY NMR spectrum of 7 in $\mathrm{CD}_{2} \mathrm{Cl}_{2}$ at a $400 \mathrm{MHz}$ spectrometer at $298 \mathrm{~K}$. 


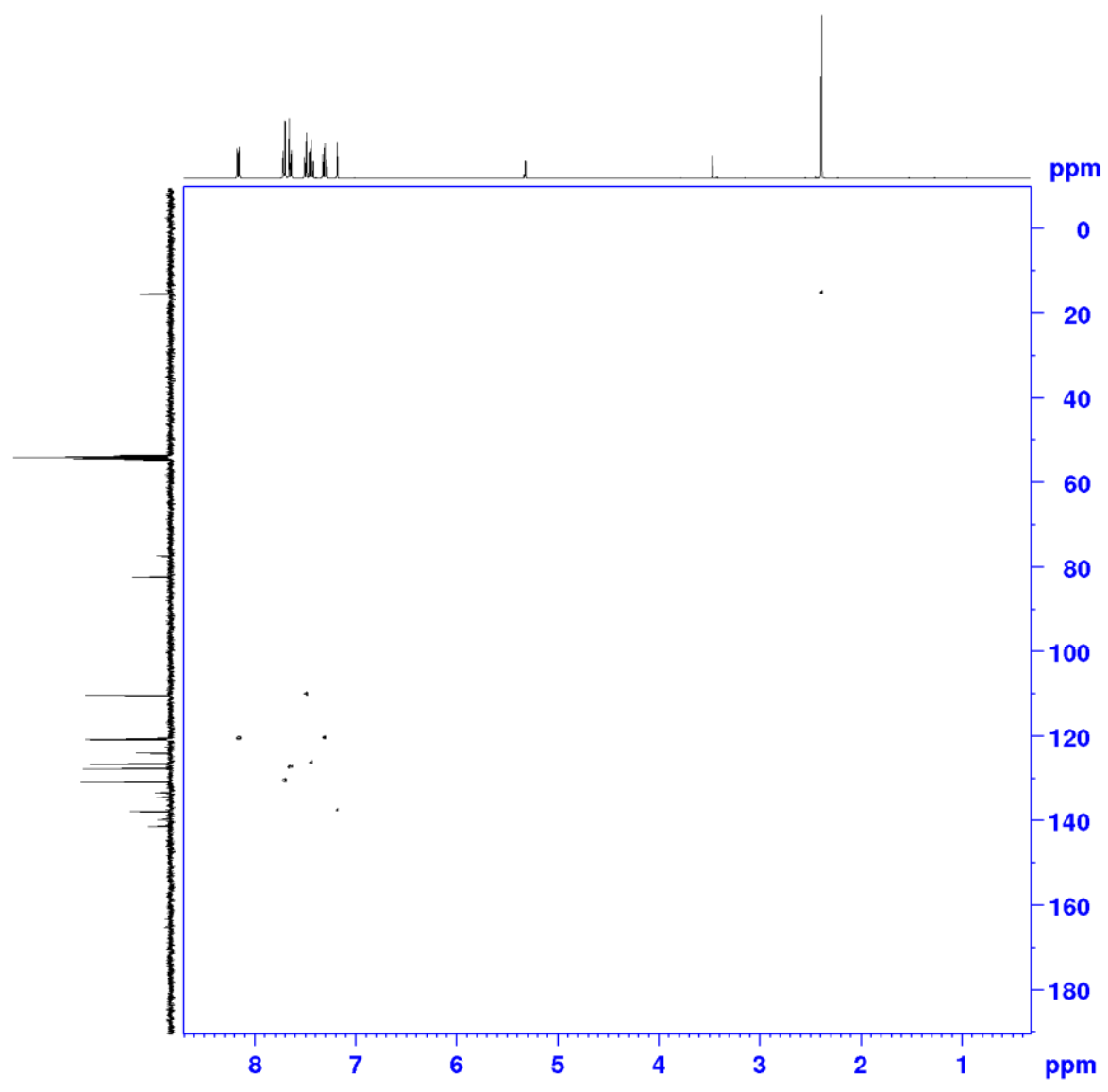

Figure S29. ${ }^{1} \mathrm{H},{ }^{13} \mathrm{C}$ gHSQC NMR spectrum of 7 in $\mathrm{CD}_{2} \mathrm{Cl}_{2}$ at a $400 \mathrm{MHz}$ spectrometer at $298 \mathrm{~K}$.

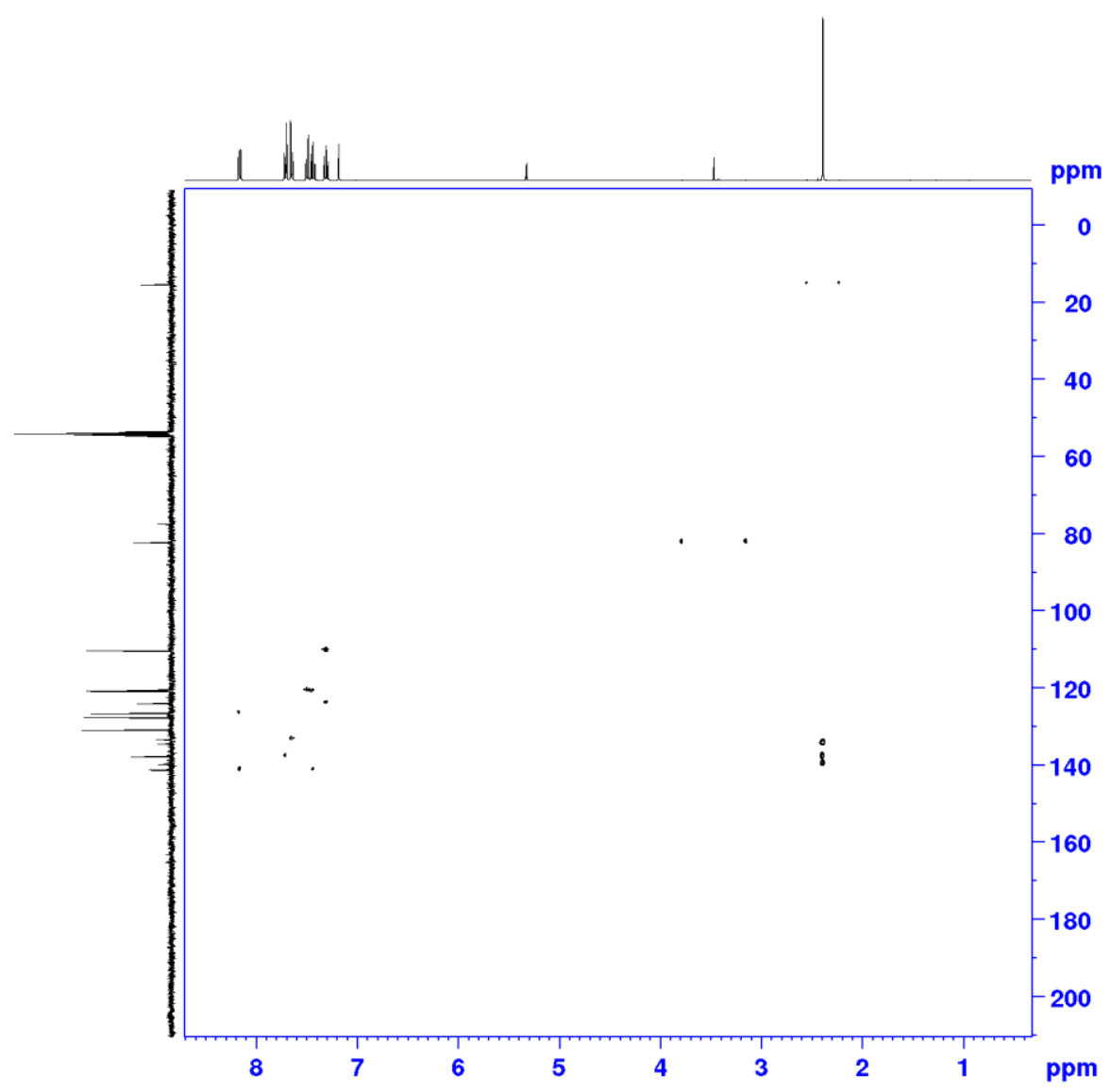

Figure S30. ${ }^{1} \mathrm{H},{ }^{13} \mathrm{C}$ gHMBC NMR spectrum of 7 in $\mathrm{CD}_{2} \mathrm{Cl}_{2}$ at a $400 \mathrm{MHz}$ spectrometer at $298 \mathrm{~K}$. 


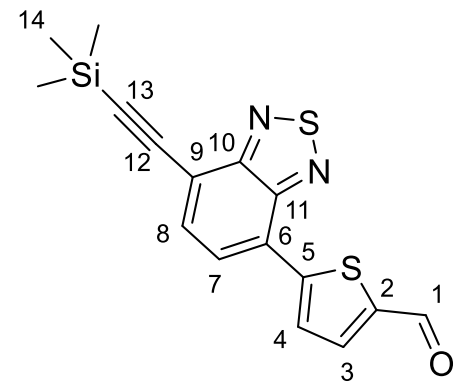

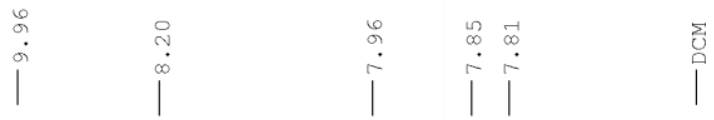

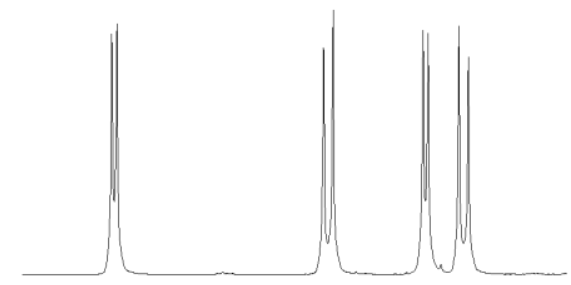

$\begin{array}{llllll}8.2 & 8.1 & 8.0 & 7.9 & 7.8 & \mathrm{ppm}\end{array}$

$|\stackrel{0}{i}|$

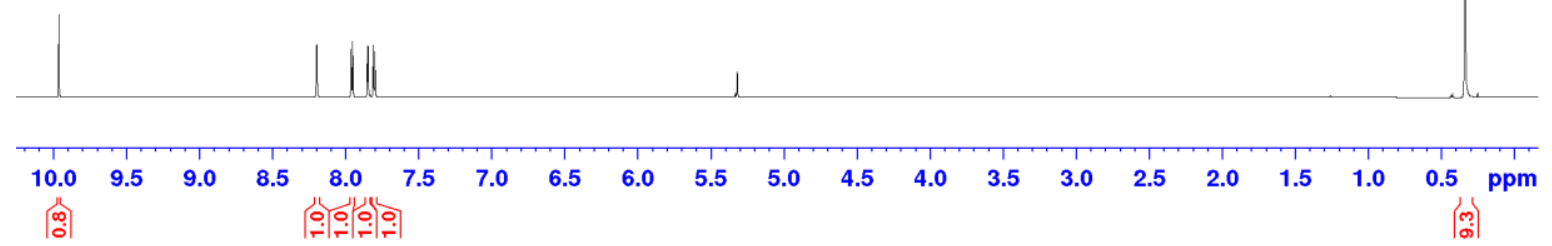

Figure S31. ${ }^{1} \mathrm{H}$ NMR of 9 in $\mathrm{CD}_{2} \mathrm{Cl}_{2}$ at a $400 \mathrm{MHz}$ spectrometer at $298 \mathrm{~K}$. 


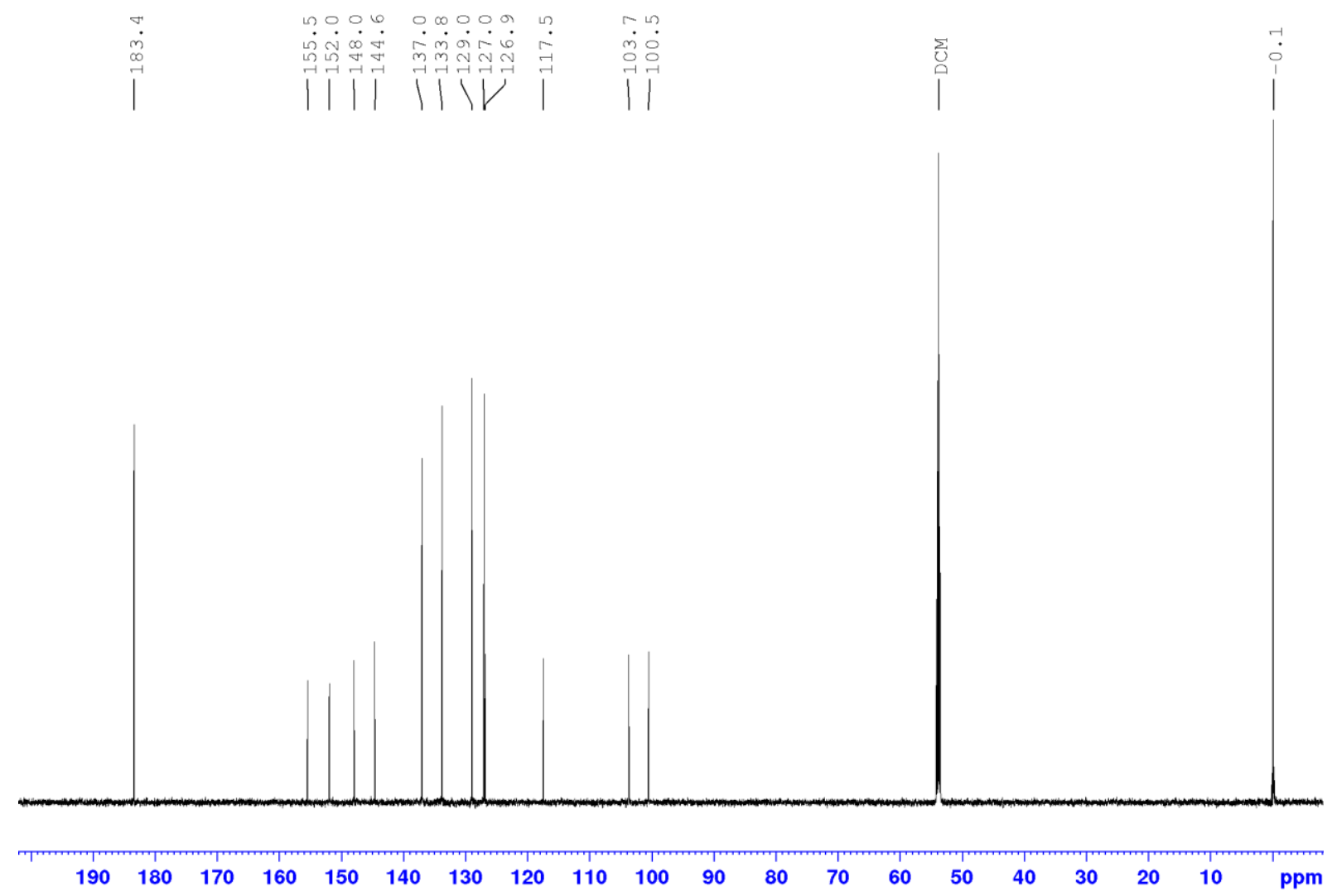

Figure S32. ${ }^{13} \mathrm{C}\left\{{ }^{1} \mathrm{H}\right\} \mathrm{NMR}$ of 9 in $\mathrm{CD}_{2} \mathrm{Cl}_{2}$ at a $100.6 \mathrm{MHz}$ spectrometer at $298 \mathrm{~K}$.

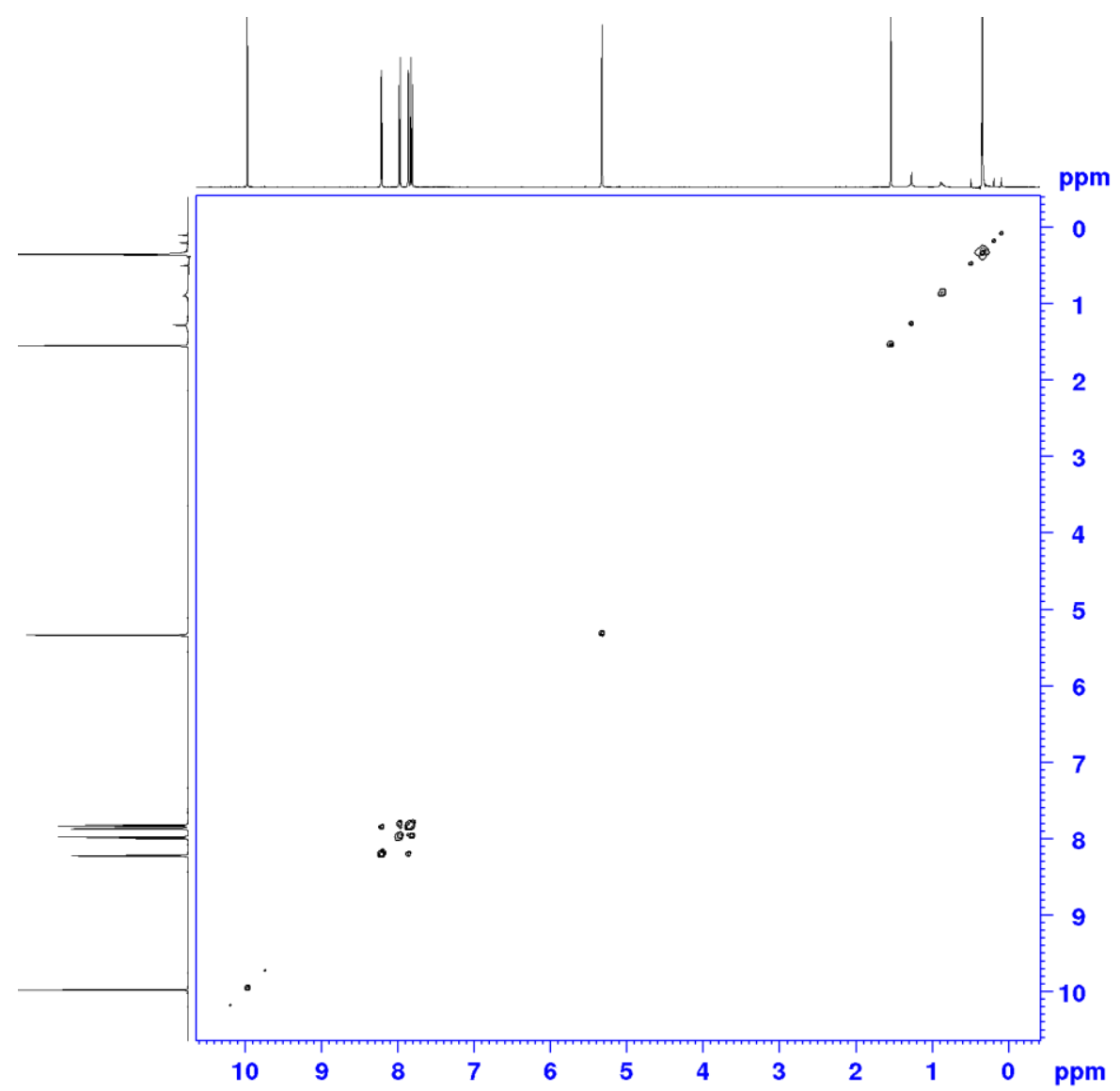

Figure $\mathbf{S 3 3}{ }^{1} \mathrm{H},{ }^{1} \mathrm{H}$ gCOSY NMR spectrum of 9 in $\mathrm{CD}_{2} \mathrm{Cl}_{2}$ at a $400 \mathrm{MHz}$ spectrometer at $298 \mathrm{~K}$. 


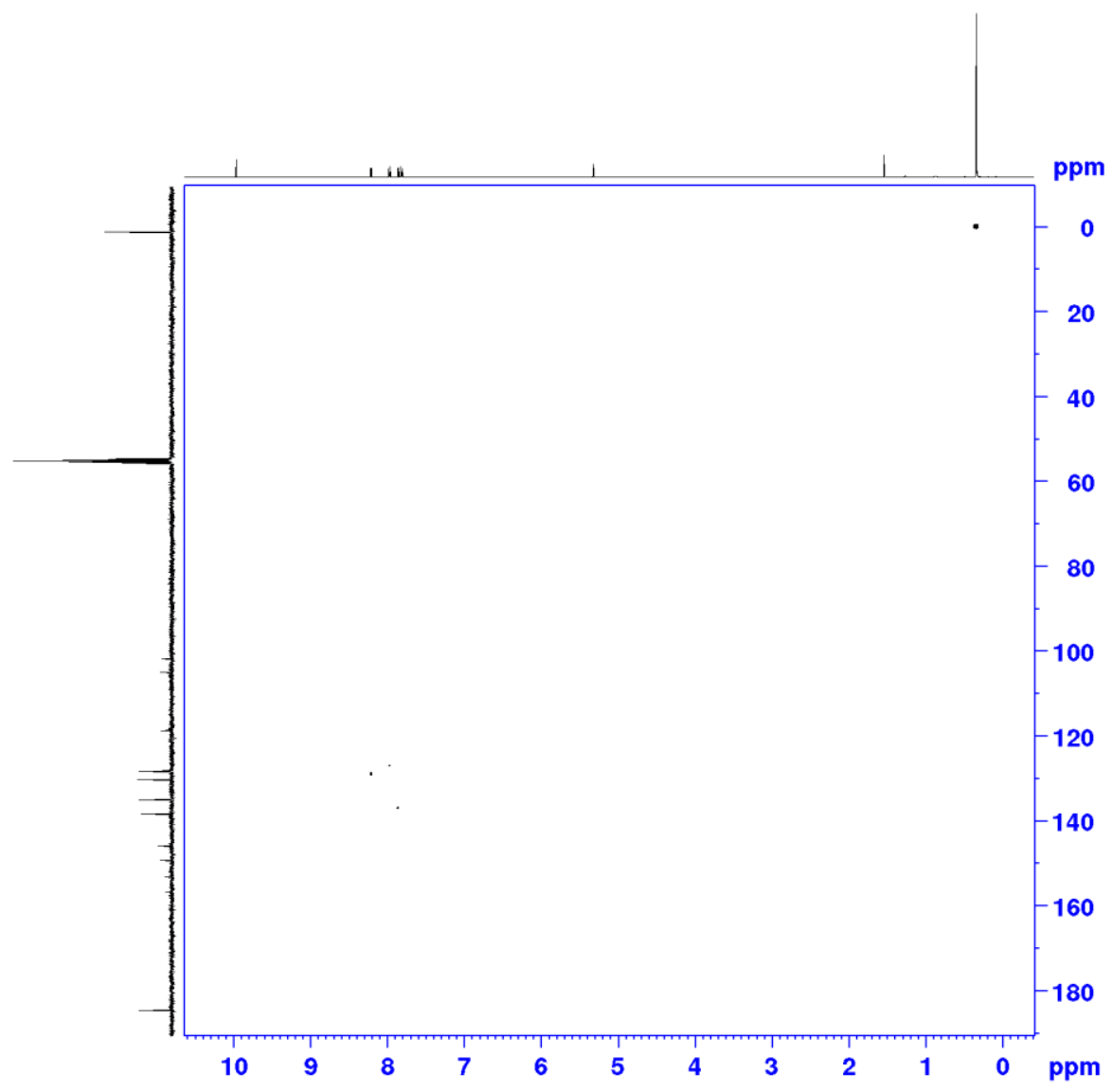

Figure S34. ${ }^{1} \mathrm{H},{ }^{13} \mathrm{C}$ gHSQC NMR spectrum of 9 in $\mathrm{CD}_{2} \mathrm{Cl}_{2}$ at a $400 \mathrm{MHz}$ spectrometer at $298 \mathrm{~K}$.

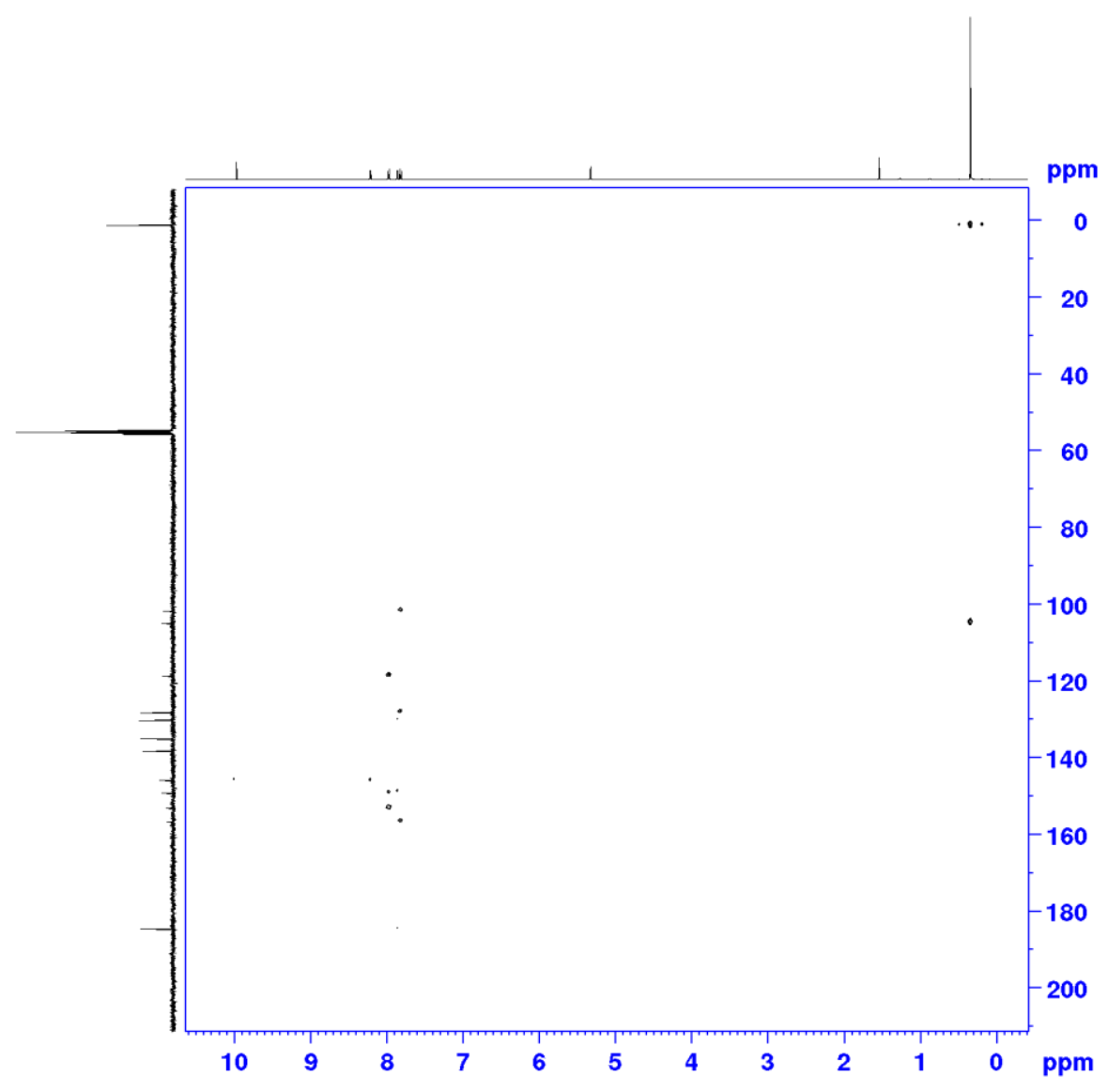

Figure S35. ${ }^{1} \mathrm{H},{ }^{13} \mathrm{C}$ gHMBC NMR spectrum of 9 in $\mathrm{CD}_{2} \mathrm{Cl}_{2}$ at a $400 \mathrm{MHz}$ spectrometer at $298 \mathrm{~K}$. 

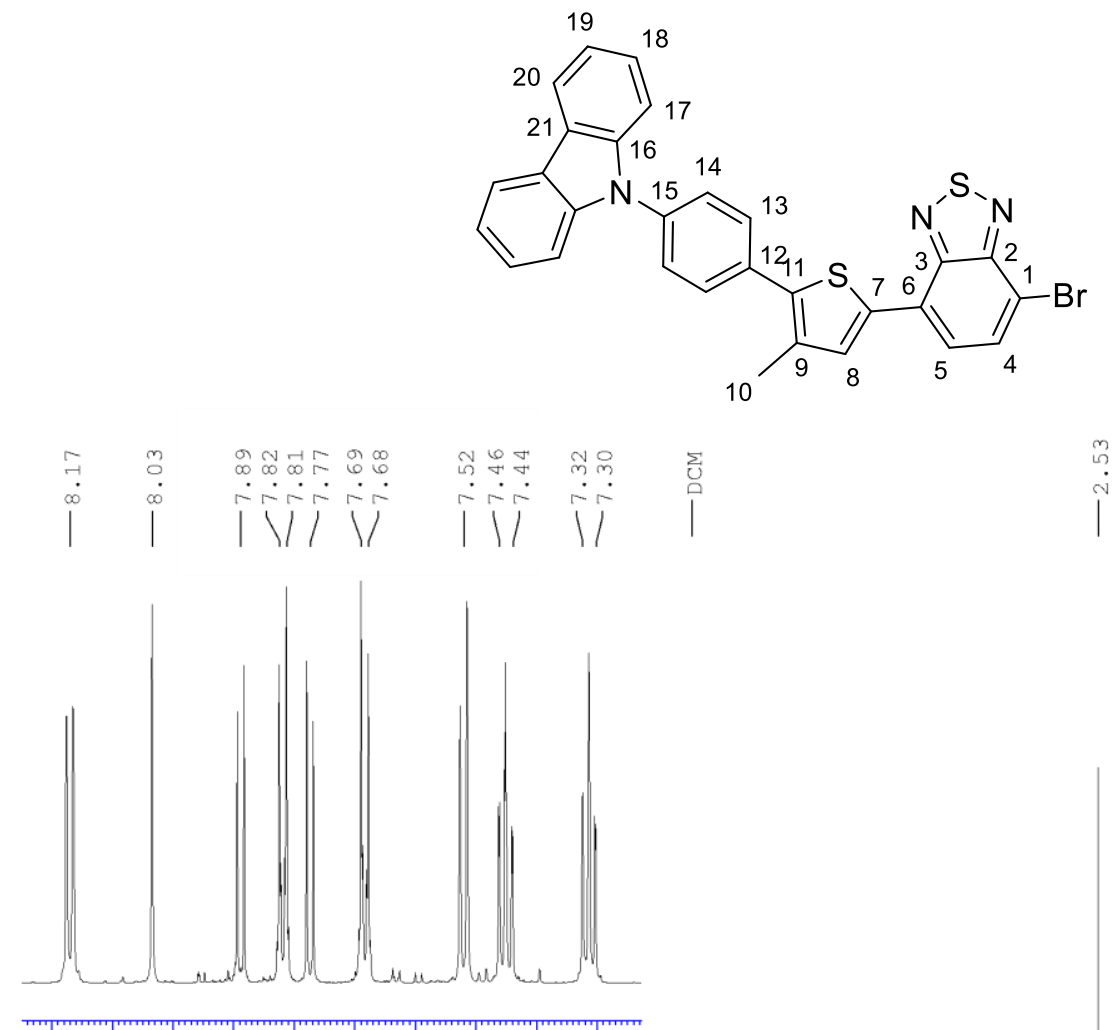

$\begin{array}{llllllllll}8.2 & 8.1 & 8.0 & 7.9 & 7.8 & 7.7 & 7.6 & 7.5 & 7.4 & \mathrm{ppm}\end{array}$

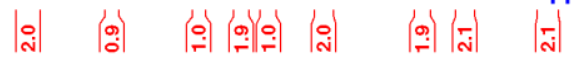

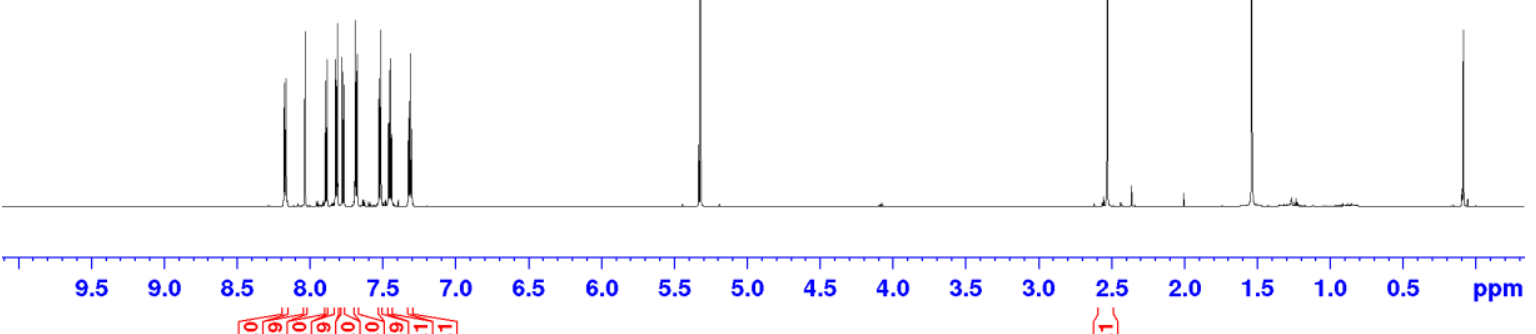

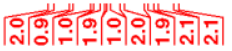

Figure S36. ${ }^{1} \mathrm{H}$ NMR of $11 \mathrm{a}$ in $\mathrm{CD}_{2} \mathrm{Cl}_{2}$ at a $700 \mathrm{MHz}$ spectrometer at $298 \mathrm{~K}$. 


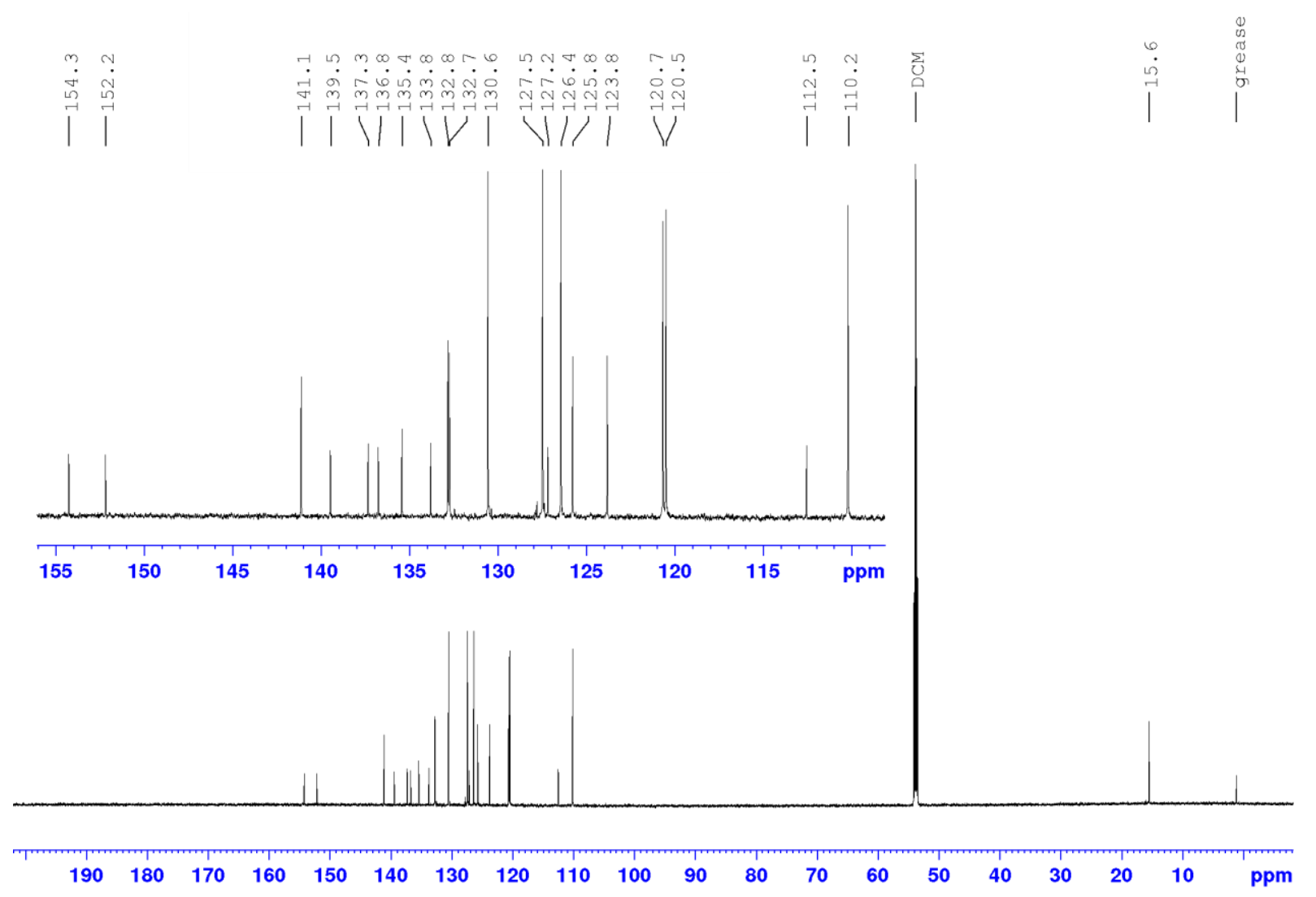

Figure S37. ${ }^{13} \mathrm{C}\left\{{ }^{1} \mathrm{H}\right\}$ NMR of 11a in $\mathrm{CD}_{2} \mathrm{Cl}_{2}$ at a $176.1 \mathrm{MHz}$ spectrometer at $298 \mathrm{~K}$.

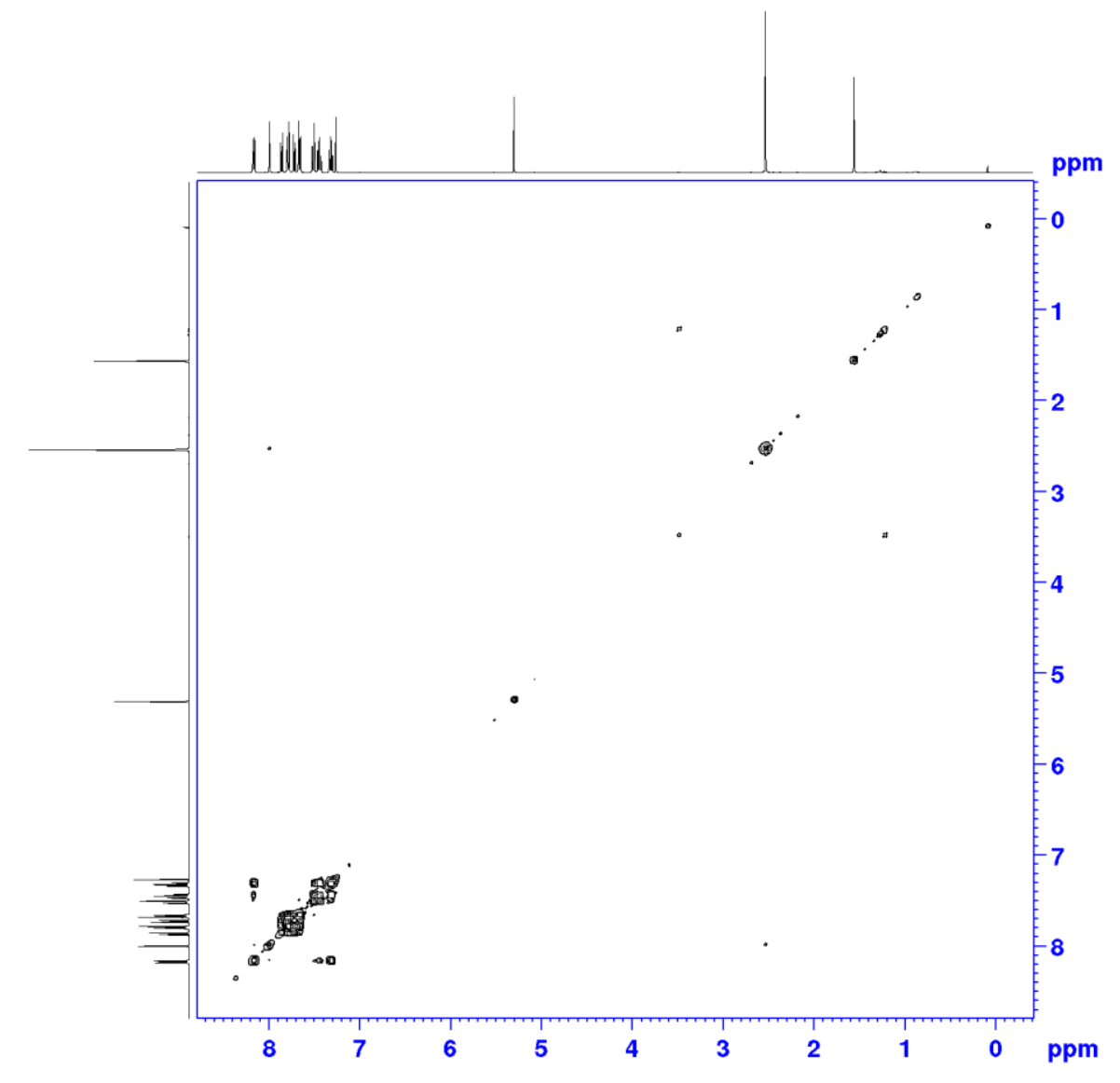

Figure S38. ${ }^{1} \mathrm{H},{ }^{1} \mathrm{H}$ gCOSY NMR spectrum of $11 a$ in $\mathrm{CDCl}_{3}$ at a $700 \mathrm{MHz}$ spectrometer at $298 \mathrm{~K}$. 


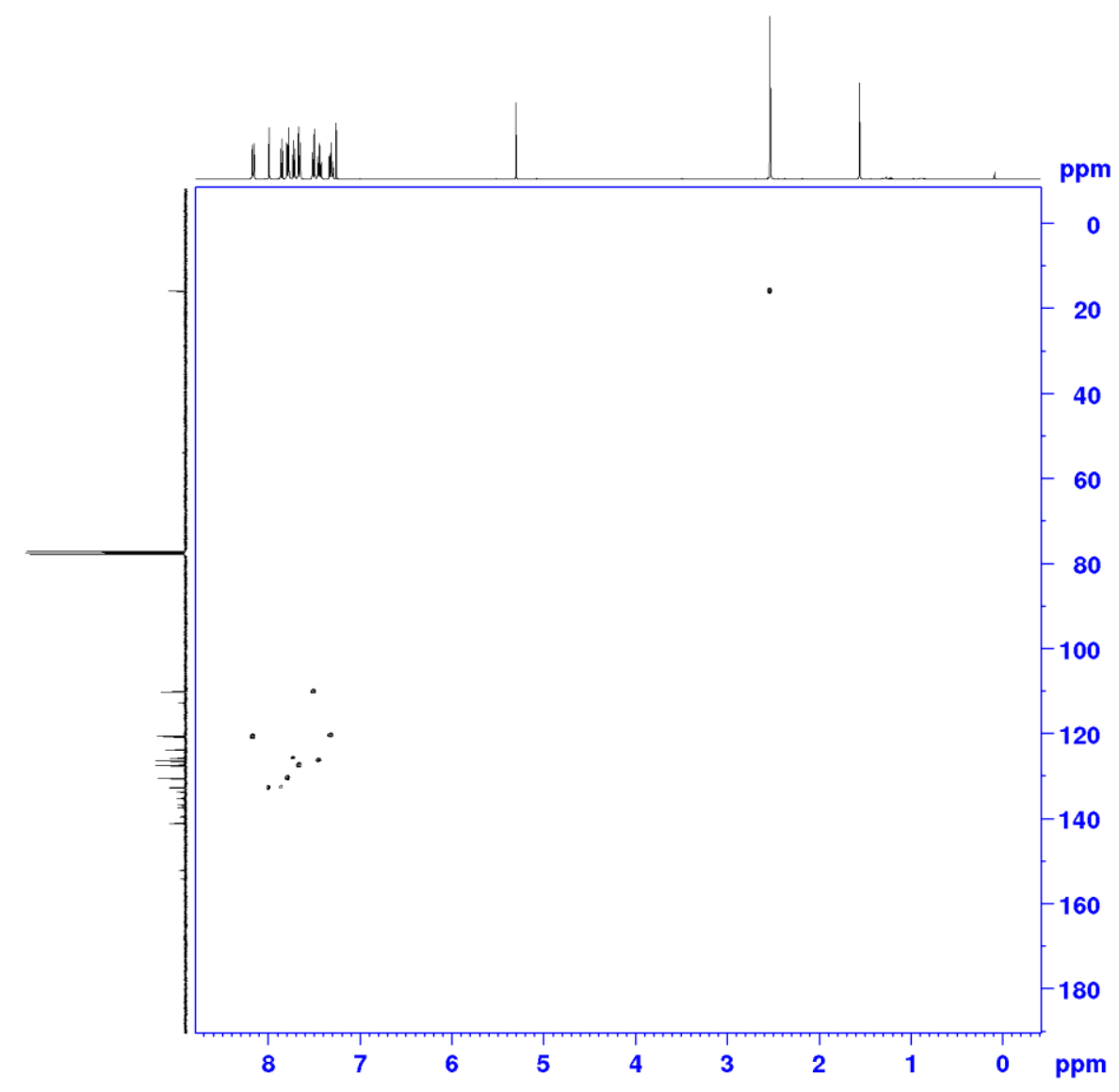

Figure S39. ${ }^{1} \mathrm{H},{ }^{13} \mathrm{C}$ gHSQC NMR spectrum of $11 \mathrm{a}$ in $\mathrm{CDCl}_{3}$ at a $700 \mathrm{MHz}$ spectrometer at $298 \mathrm{~K}$.

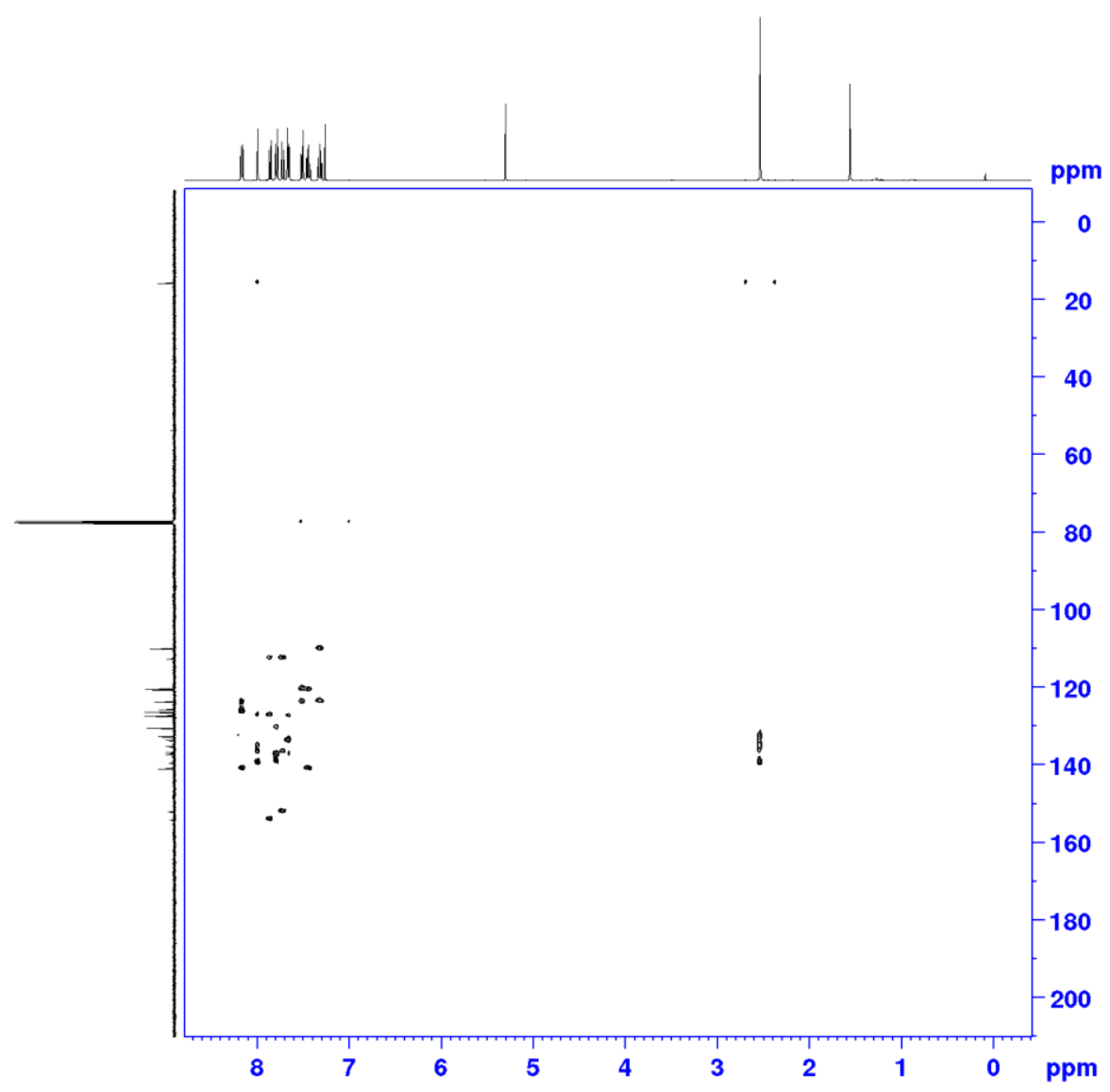

Figure S40. ${ }^{1} \mathrm{H},{ }^{13} \mathrm{C}$ gHMBC NMR spectrum of $\mathbf{1 1 a}$ in $\mathrm{CDCl}_{3}$ at a $700 \mathrm{MHz}$ spectrometer at $298 \mathrm{~K}$. 


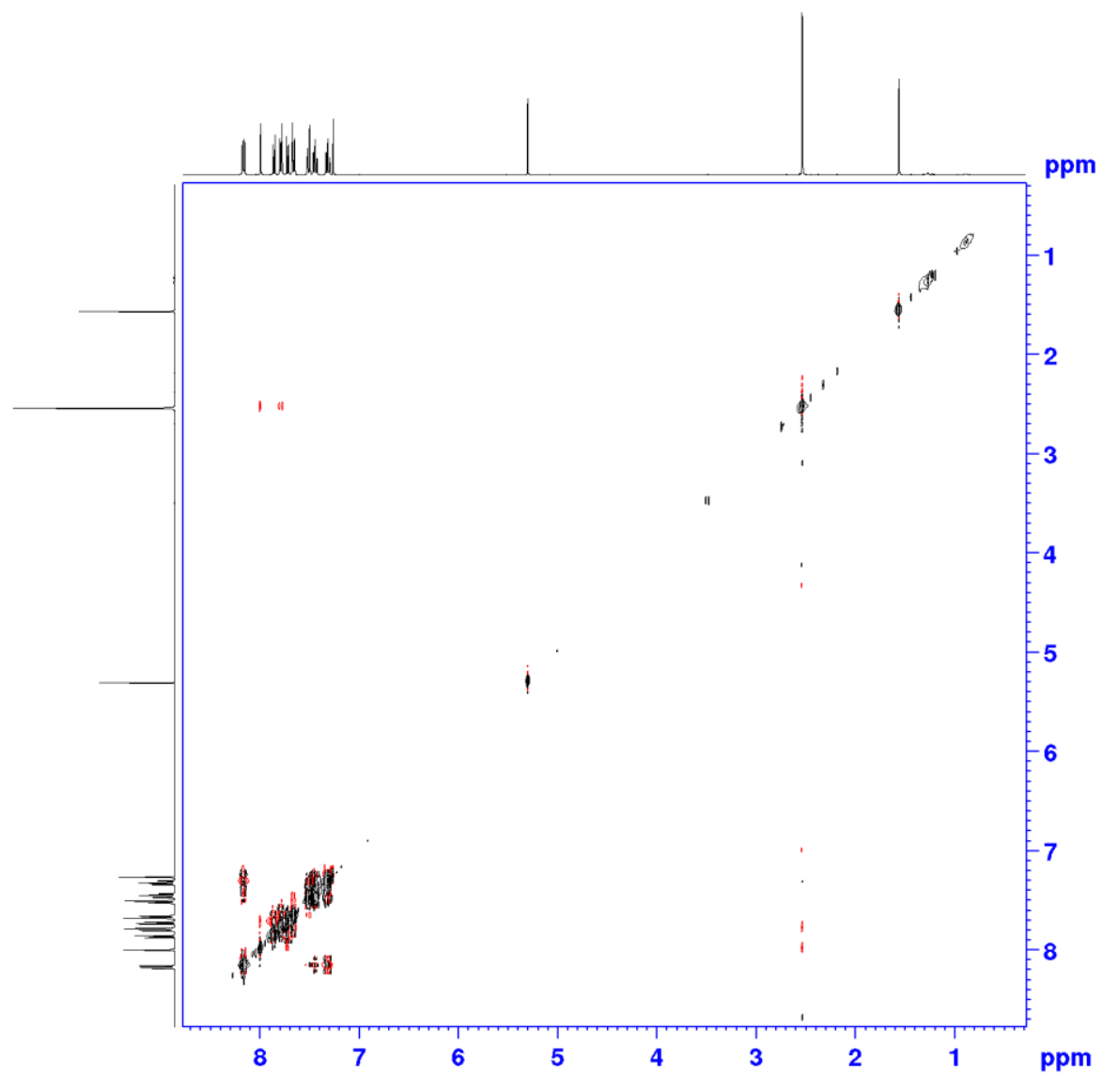

Figure S41. ${ }^{1} \mathrm{H},{ }^{1} \mathrm{H}$ gNOESY NMR spectrum of $11 \mathrm{a}$ in $\mathrm{CDCl}_{3}$ at a $700 \mathrm{MHz}$ spectrometer at $298 \mathrm{~K}$. 

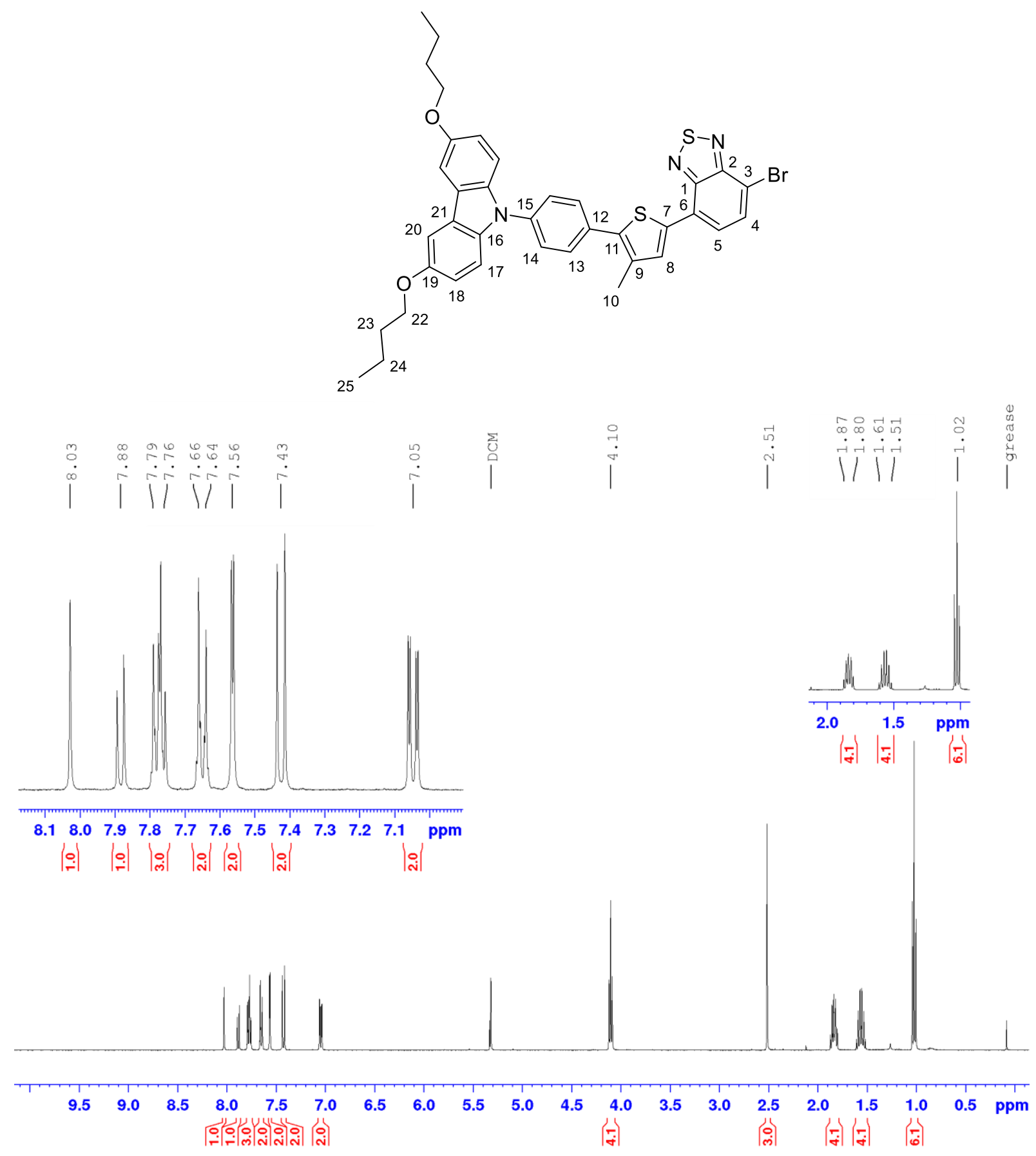

Figure S42. ${ }^{1} \mathrm{H}$ NMR of $\mathbf{1 1 b}$ in $\mathrm{CD}_{2} \mathrm{Cl}_{2}$ at a $400 \mathrm{MHz}$ spectrometer at $298 \mathrm{~K}$. 


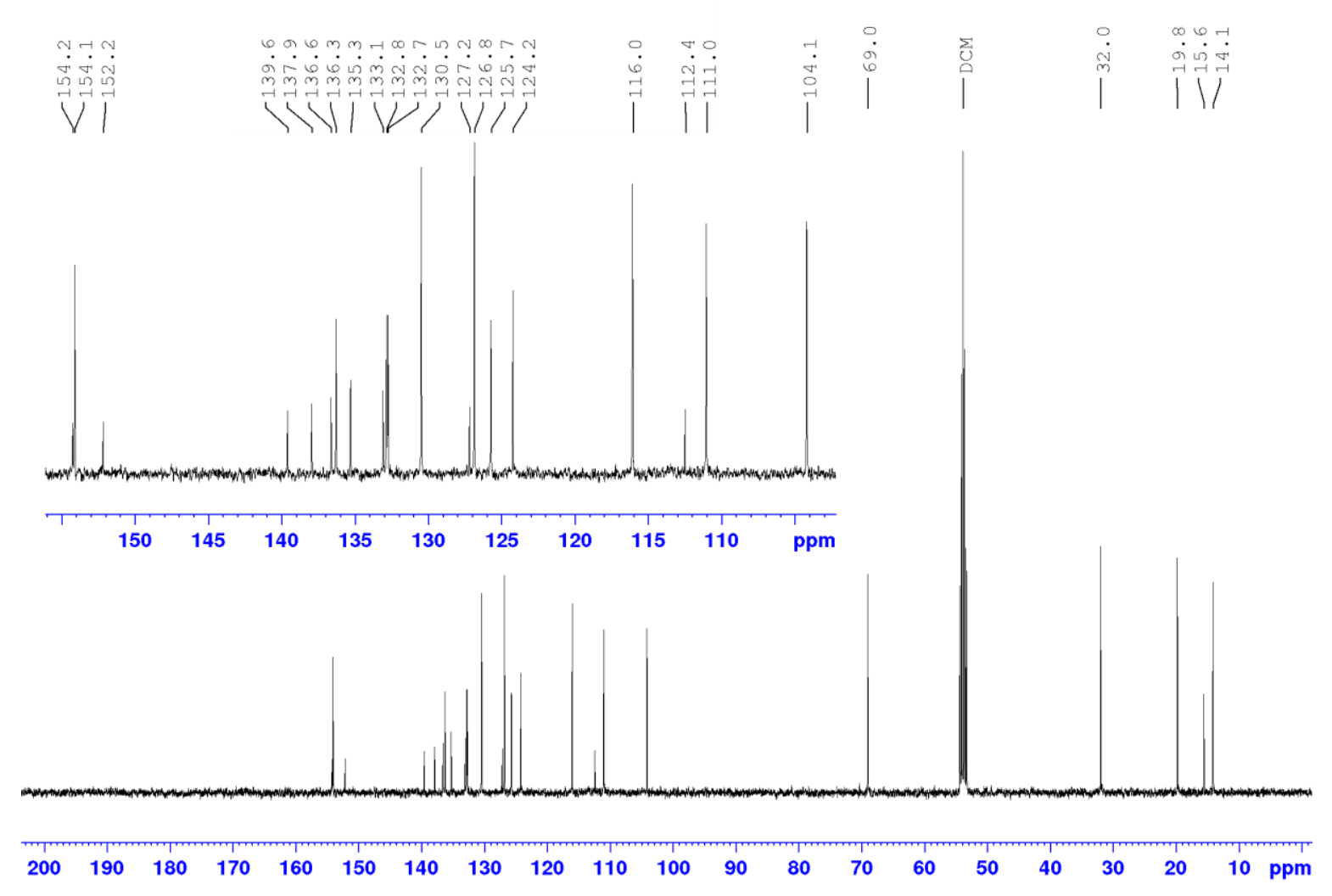

Figure S43. ${ }^{13} \mathrm{C}\left\{{ }^{1} \mathrm{H}\right\}$ NMR of $\mathbf{1 1 b}$ in $\mathrm{CD}_{2} \mathrm{Cl}_{2}$ at a $100.6 \mathrm{MHz}$ spectrometer at $298 \mathrm{~K}$.

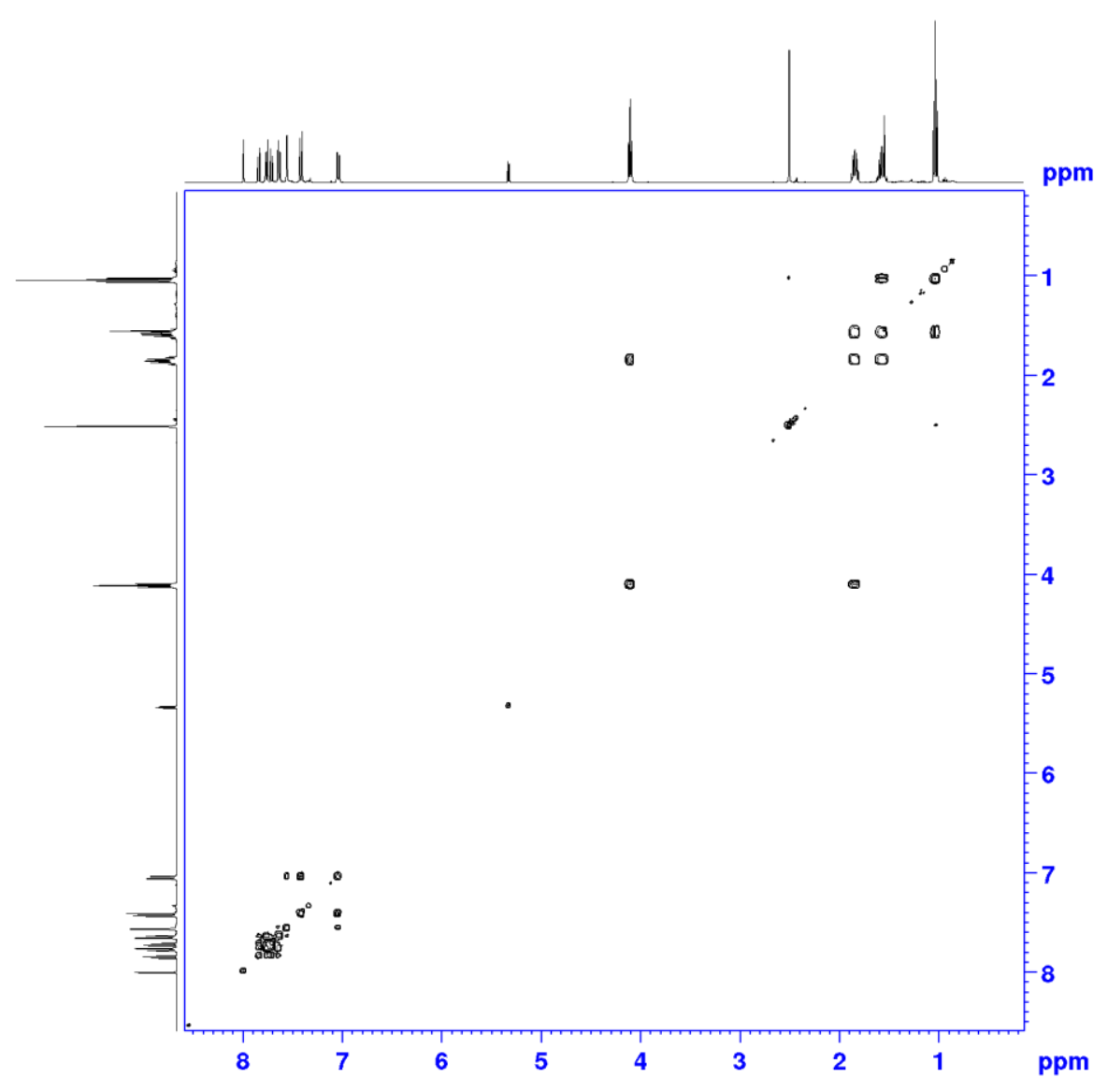

Figure S44. ${ }^{1} \mathrm{H},{ }^{1} \mathrm{H}$ gCOSY NMR spectrum of $\mathbf{1 1 b}$ in $\mathrm{CD}_{2} \mathrm{Cl}_{2}$ at a $400 \mathrm{MHz}$ spectrometer at $298 \mathrm{~K}$. 


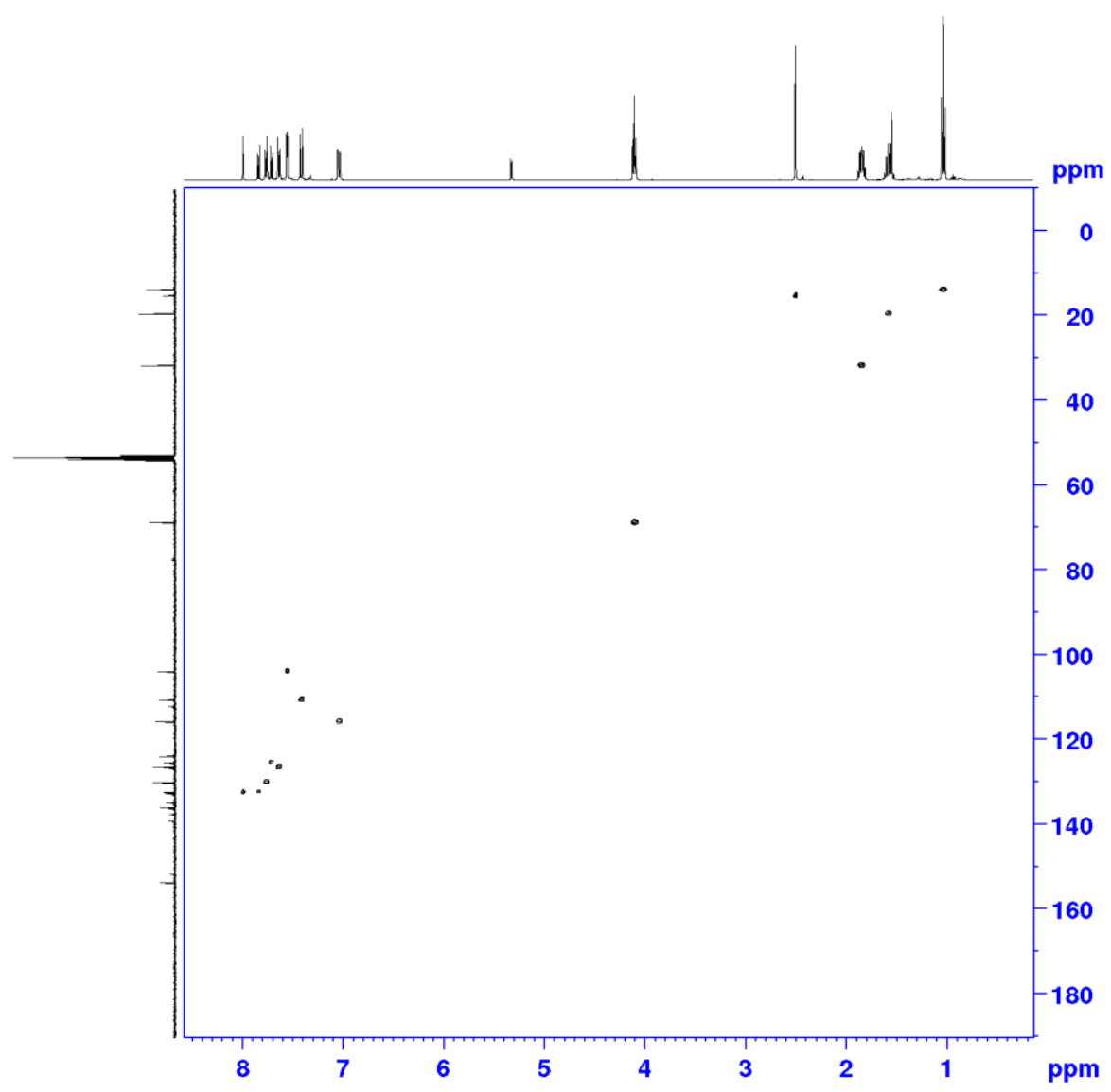

Figure S45. ${ }^{1} \mathrm{H},{ }^{13} \mathrm{C}$ gHSQC NMR spectrum of $\mathbf{1 1 b}$ in $\mathrm{CD}_{2} \mathrm{Cl}_{2}$ at a $400 \mathrm{MHz}$ spectrometer at $298 \mathrm{~K}$.

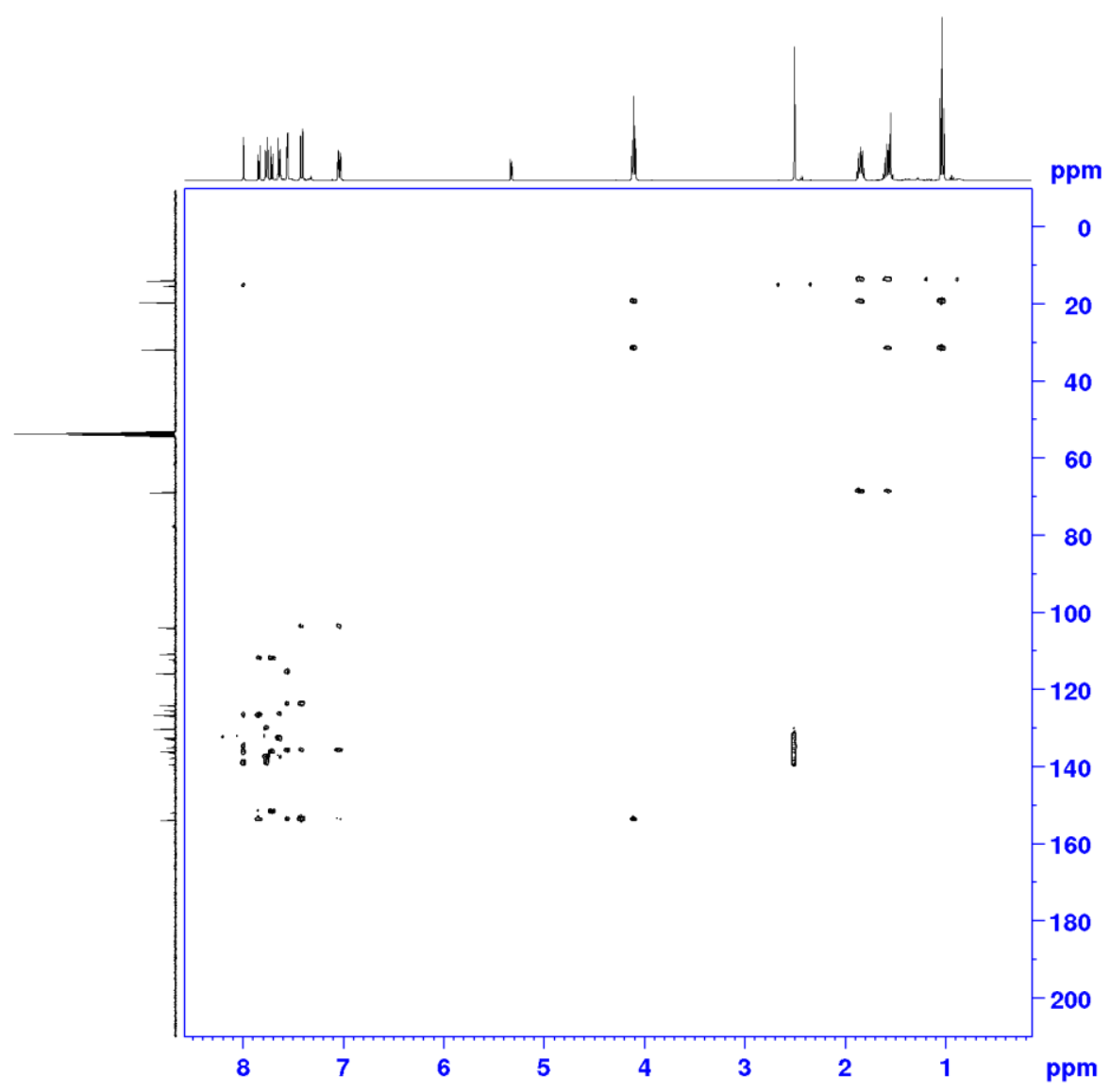

Figure S46. ${ }^{1} \mathrm{H},{ }^{13} \mathrm{C}$ gHMBC NMR spectrum of $\mathbf{1 1 b}$ in $\mathrm{CD}_{2} \mathrm{Cl}_{2}$ at a $400 \mathrm{MHz}$ spectrometer at $298 \mathrm{~K}$. 

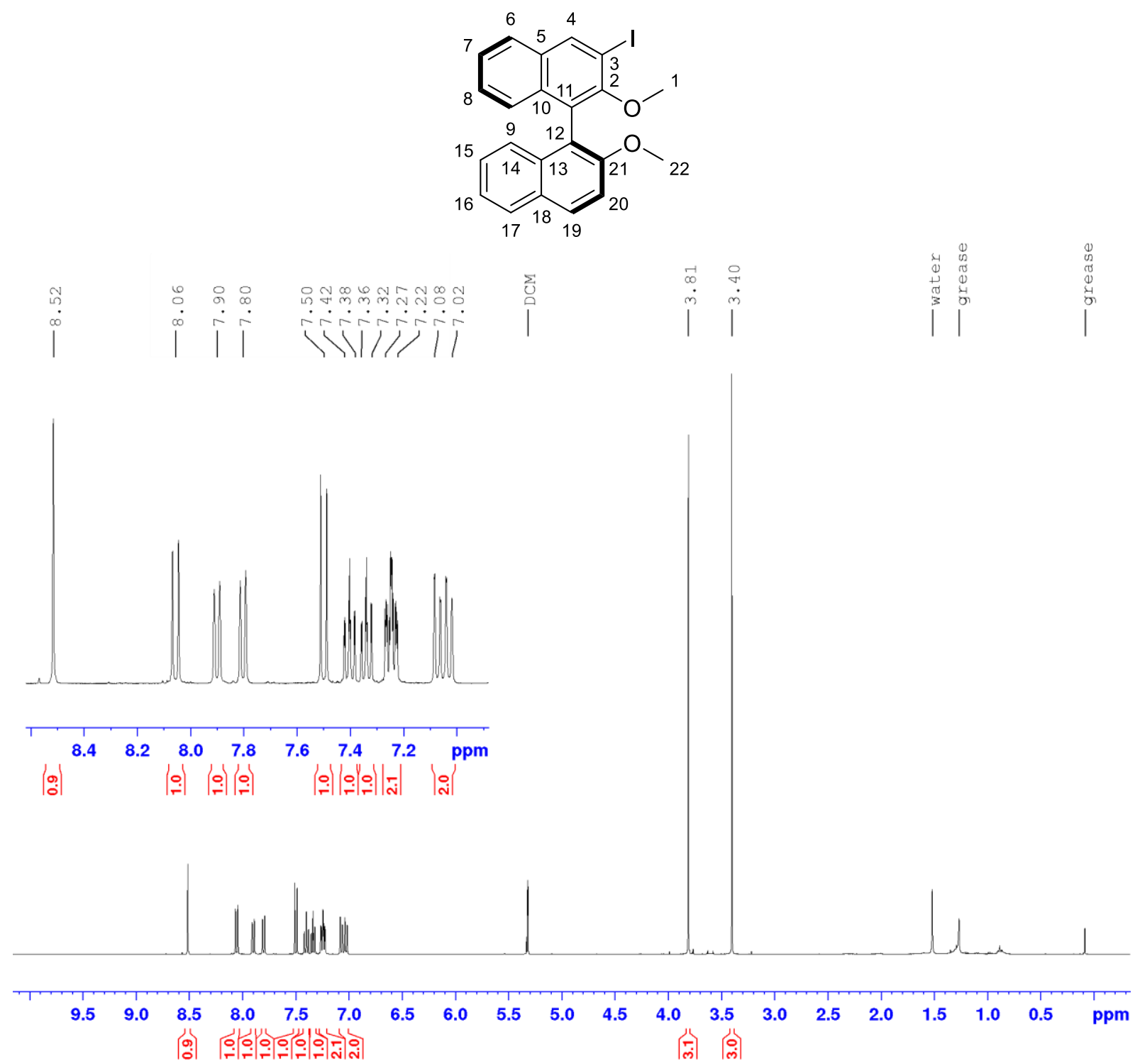

Figure S47. ${ }^{1} \mathrm{H}$ NMR of $\mathbf{1 3}(S)$ b in $\mathrm{CD}_{2} \mathrm{Cl}_{2}$ at a $400 \mathrm{MHz}$ spectrometer at $298 \mathrm{~K}$. 


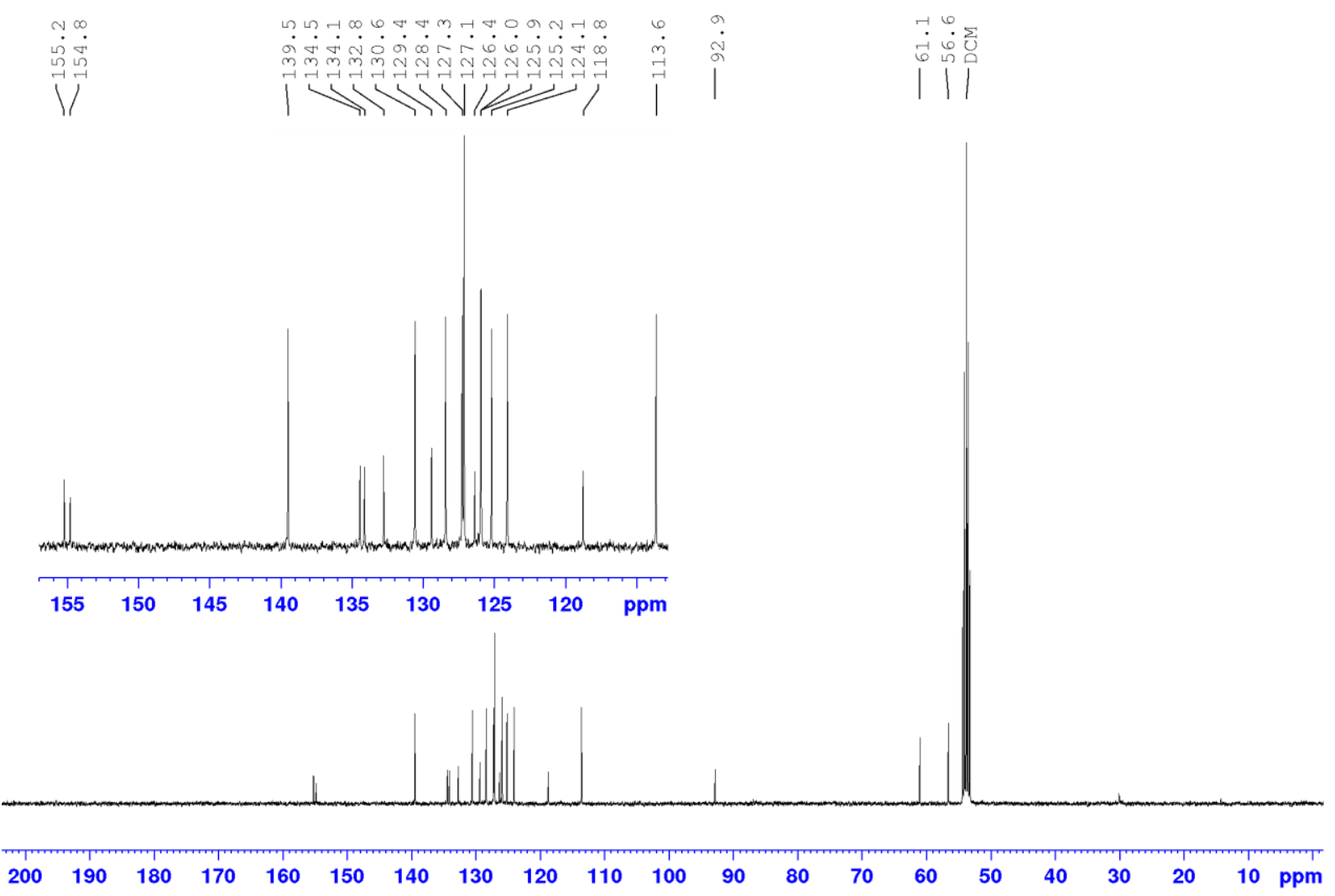

Figure S48. ${ }^{13} \mathrm{C}\left\{{ }^{1} \mathrm{H}\right\}$ NMR of $\mathbf{1 3}(S)$ b in $\mathrm{CD}_{2} \mathrm{Cl}_{2}$ at a $100.6 \mathrm{MHz}$ spectrometer at $298 \mathrm{~K}$. 

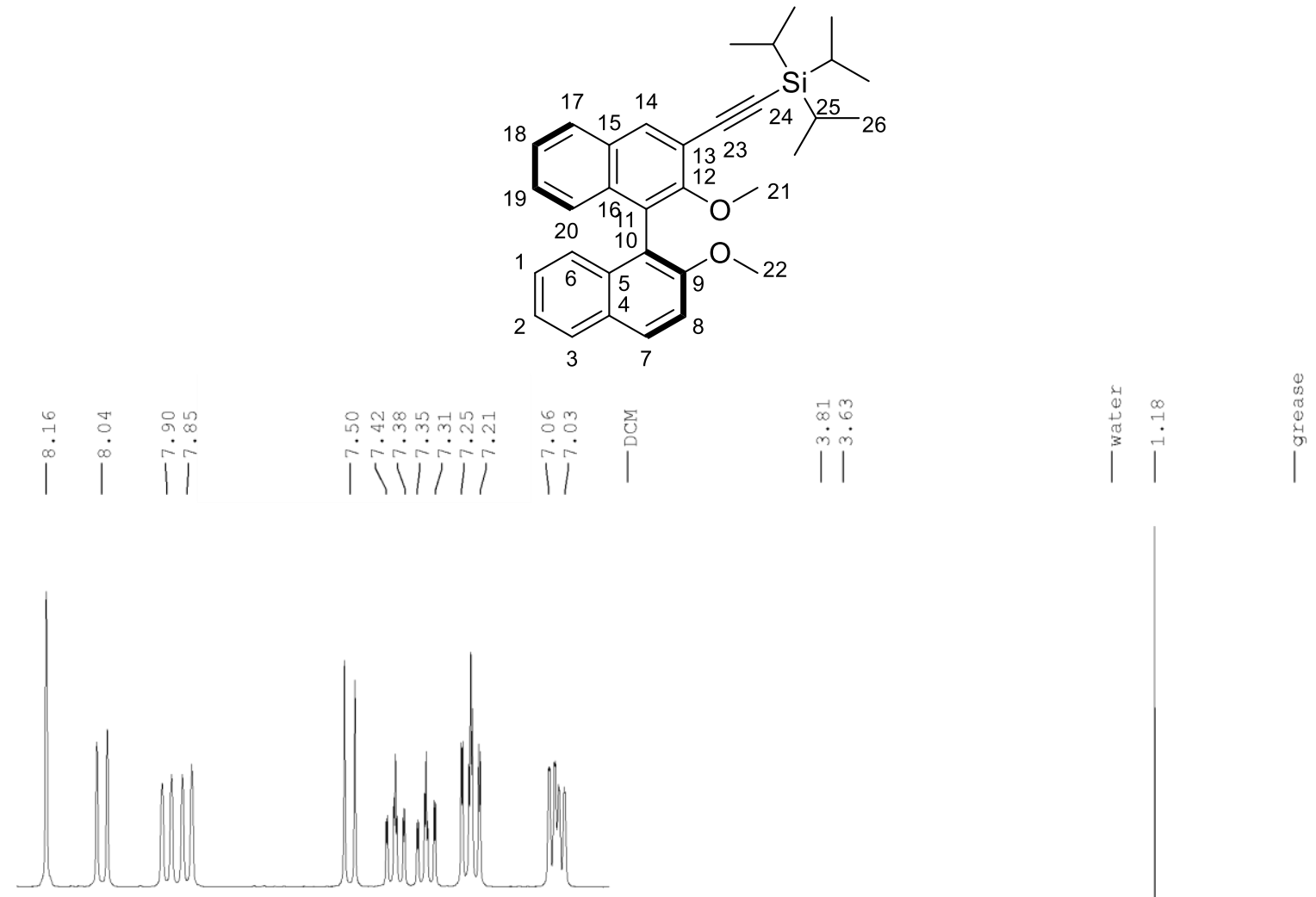

$\begin{array}{llllllllllll}8.1 & 8.0 & 7.9 & 7.8 & 7.7 & 7.6 & 7.5 & 7.4 & 7.3 & 7.2 & 7.1 & \text { ppm }\end{array}$

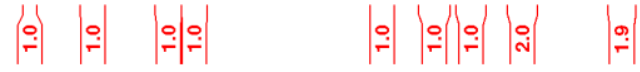

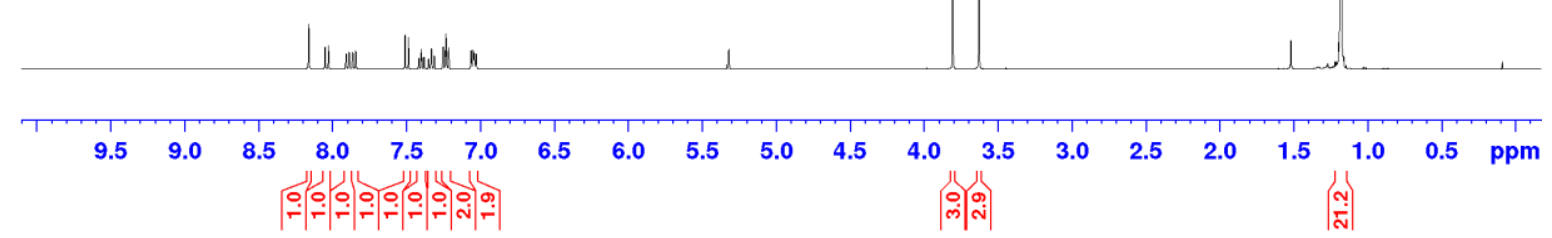

Figure S49. ${ }^{1} \mathrm{H}$ NMR of $\mathbf{1 4}(S)$ in $\mathrm{CD}_{2} \mathrm{Cl}_{2}$ at a $400 \mathrm{MHz}$ spectrometer at $298 \mathrm{~K}$. 

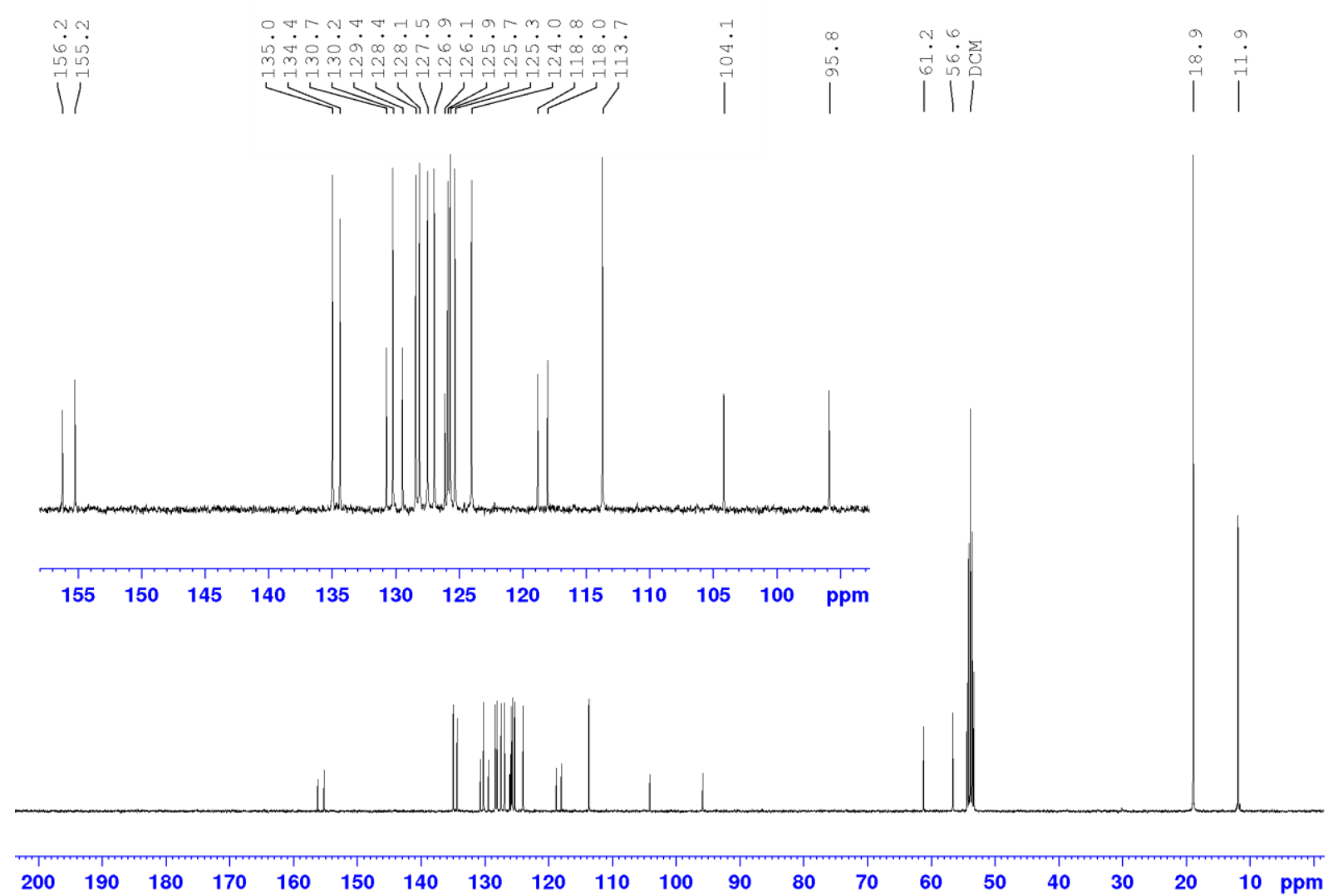

Figure S50. ${ }^{13} \mathrm{C}\left\{{ }^{1} \mathrm{H}\right\} \mathrm{NMR}$ of $\mathbf{1 4}(\mathrm{S})$ in $\mathrm{CD}_{2} \mathrm{Cl}_{2}$ at a $100.6 \mathrm{MHz}$ spectrometer at $298 \mathrm{~K}$.

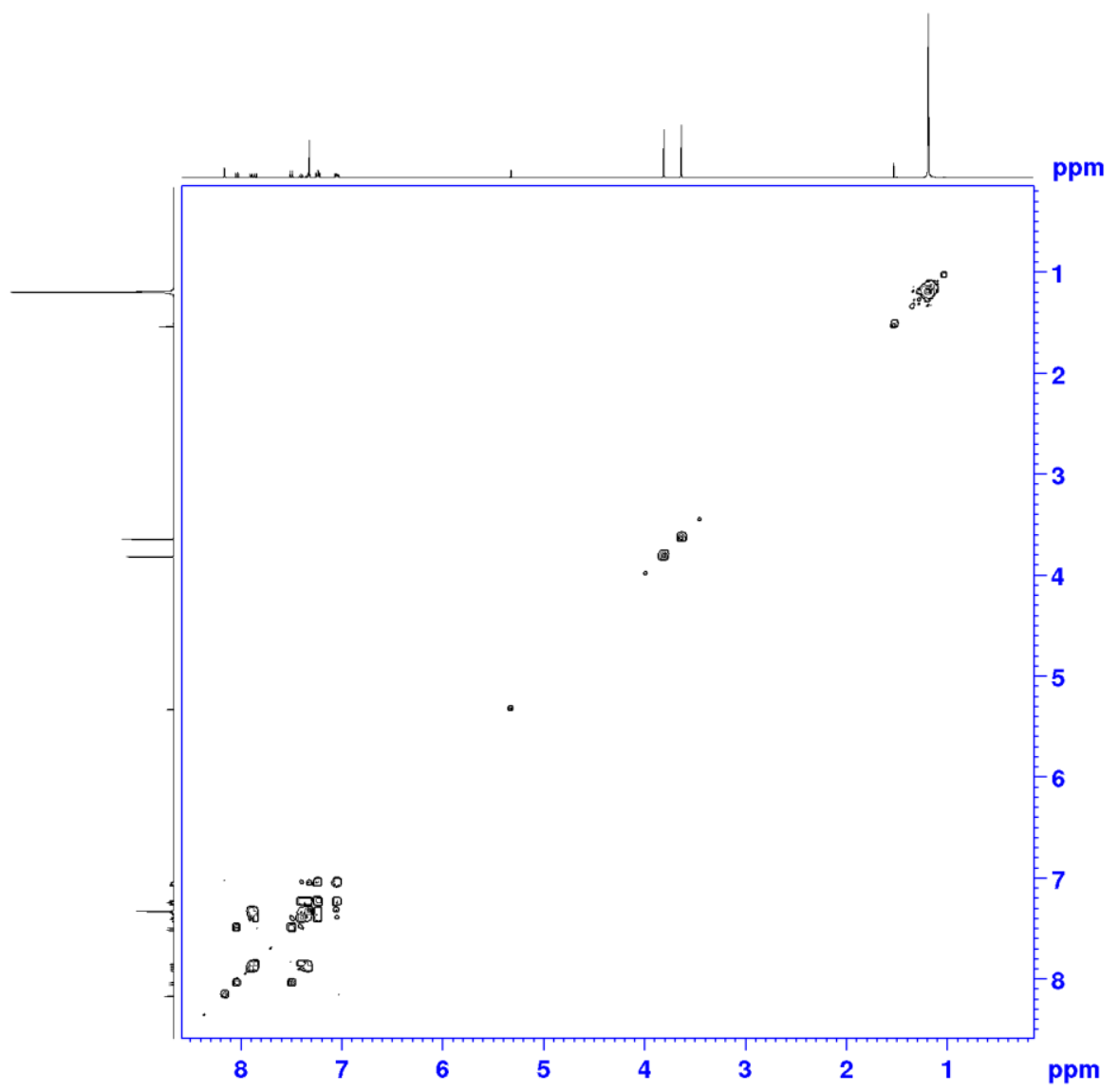

Figure S51. ${ }^{1} \mathrm{H},{ }^{1} \mathrm{H}$ gCOSY NMR spectrum of $\mathbf{1 4}(S)$ in $\mathrm{CD}_{2} \mathrm{Cl}_{2}$ at a $400 \mathrm{MHz}$ spectrometer at $298 \mathrm{~K}$. 


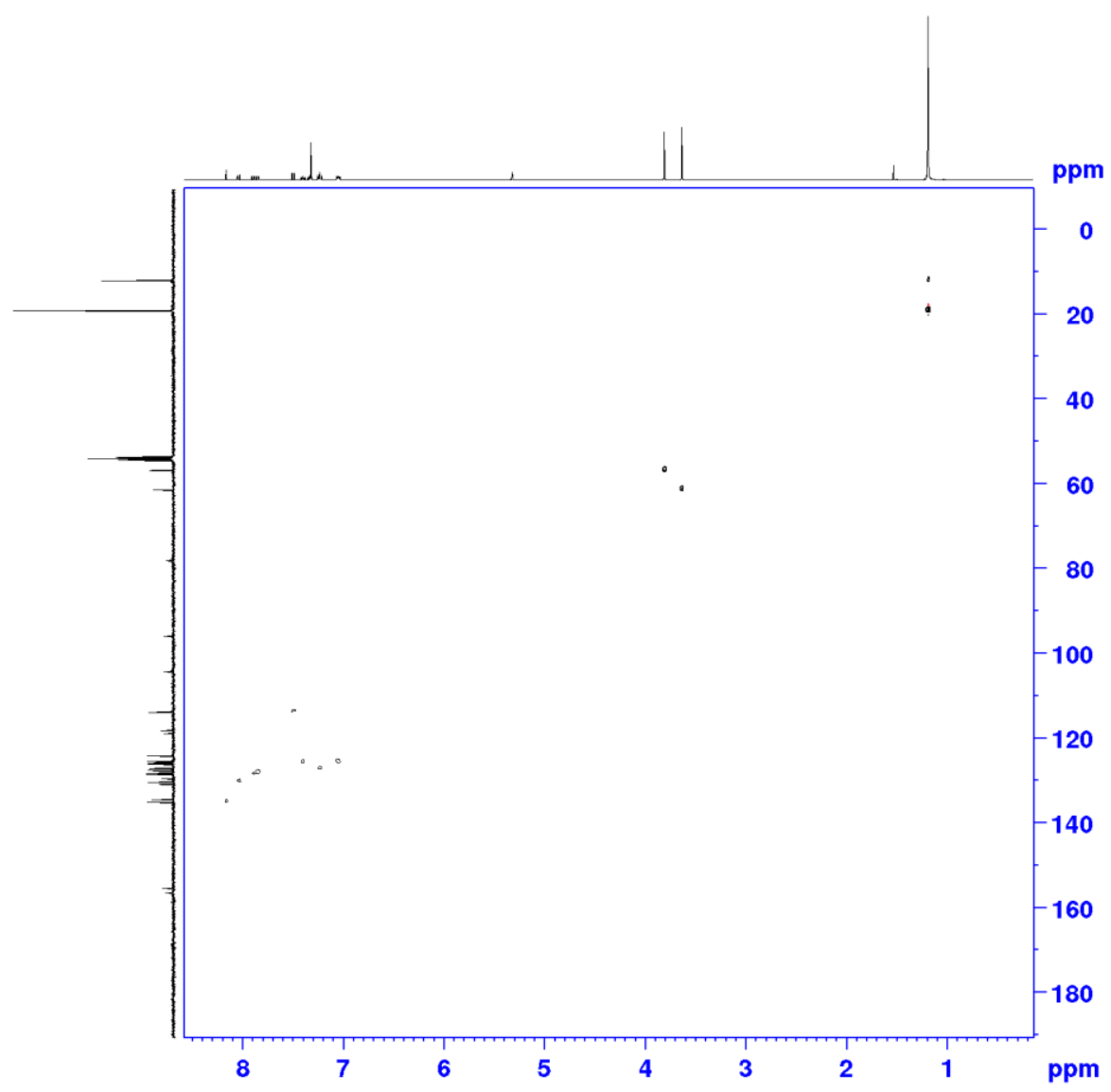

Figure S52. ${ }^{1} \mathrm{H},{ }^{13} \mathrm{C}$ gHSQC NMR spectrum of $\mathbf{1 4}(S)$ in $\mathrm{CD}_{2} \mathrm{Cl}_{2}$ at a $400 \mathrm{MHz}$ spectrometer at $298 \mathrm{~K}$.

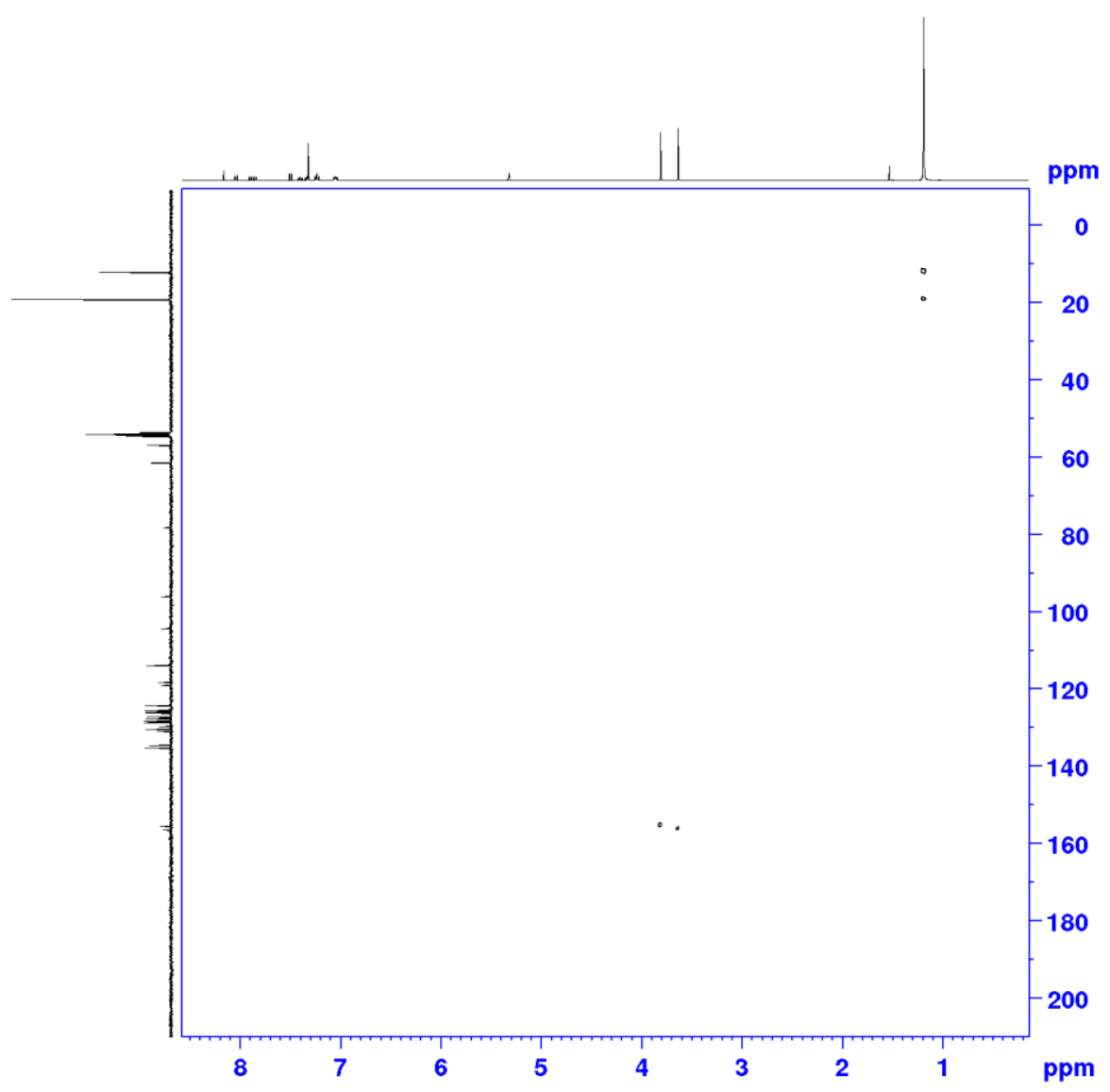

Figure S53. ${ }^{1} \mathrm{H},{ }^{13} \mathrm{C}$ gHMBC NMR spectrum of $\mathbf{1 4}(\boldsymbol{S})$ in $\mathrm{CD}_{2} \mathrm{Cl}_{2}$ at a $400 \mathrm{MHz}$ spectrometer at $298 \mathrm{~K}$. 

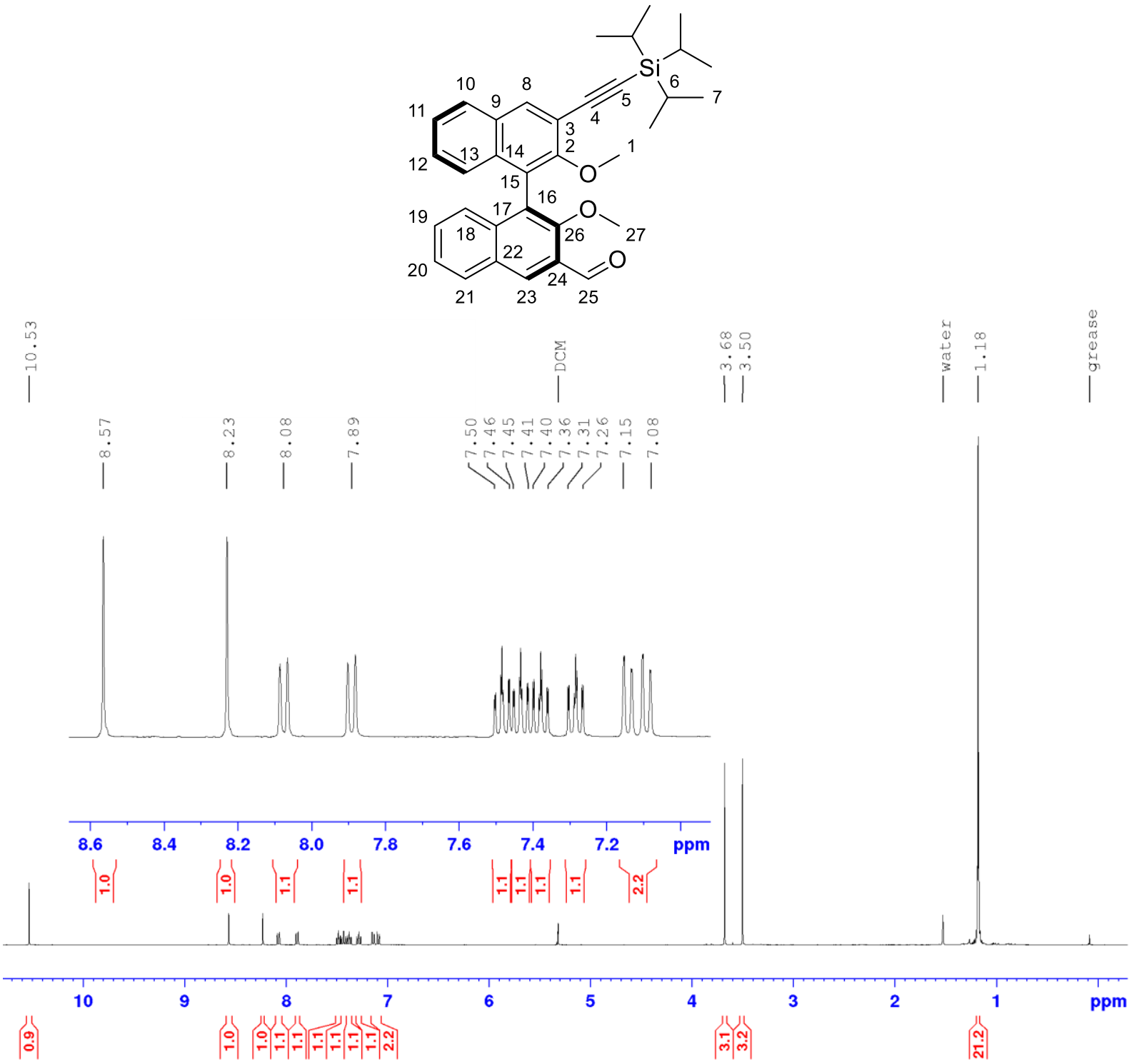

Figure S54. ${ }^{1} \mathrm{H}$ NMR of $\mathbf{1 5}(\boldsymbol{S})$ in $\mathrm{CD}_{2} \mathrm{Cl}_{2}$ at a $400 \mathrm{MHz}$ spectrometer at $298 \mathrm{~K}$. 


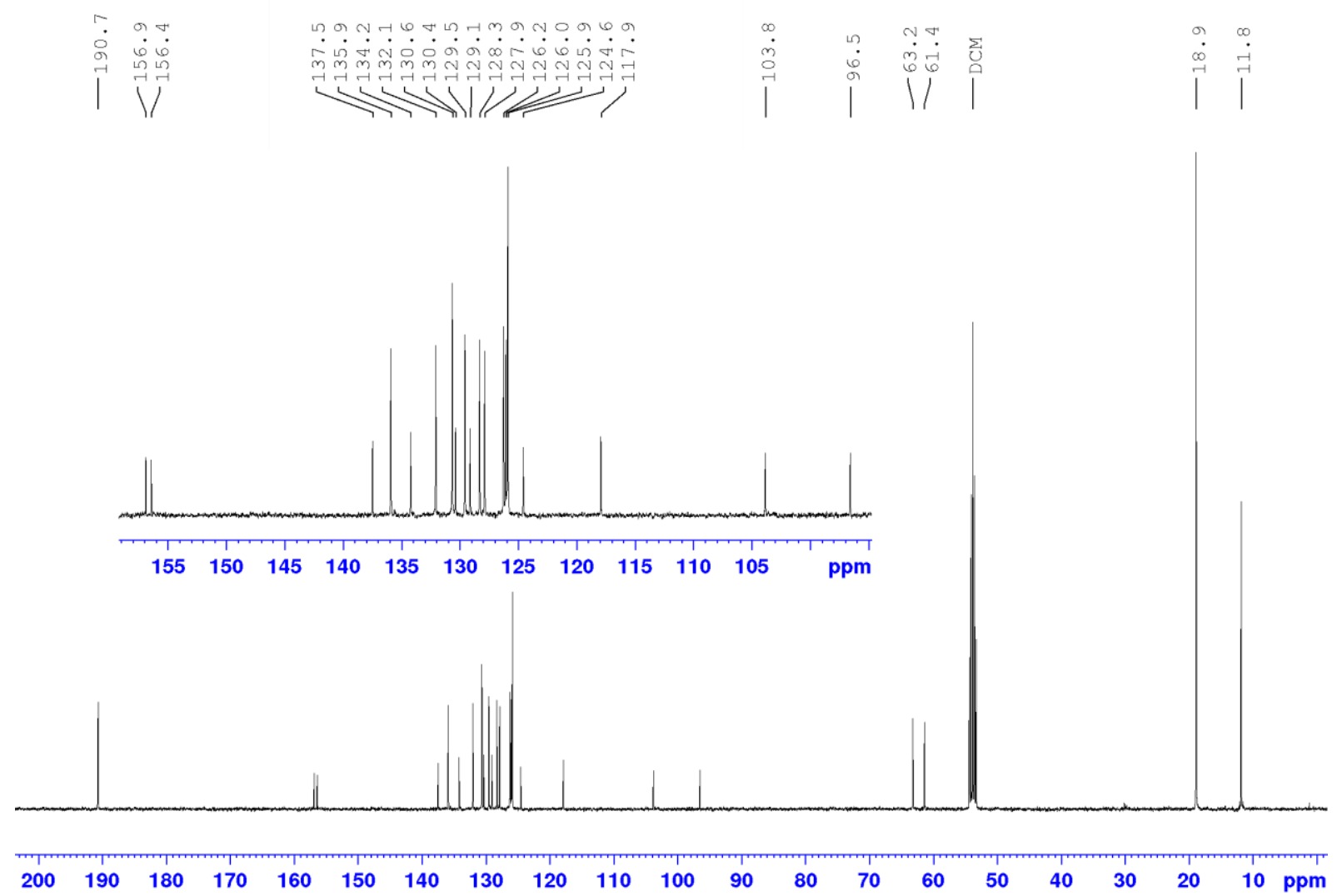

Figure S55. ${ }^{13} \mathrm{C}\left\{{ }^{1} \mathrm{H}\right\} \mathrm{NMR}$ of $\mathbf{1 5}(\boldsymbol{S})$ in $\mathrm{CD}_{2} \mathrm{Cl}_{2}$ at a $100.6 \mathrm{MHz}$ spectrometer at $298 \mathrm{~K}$.

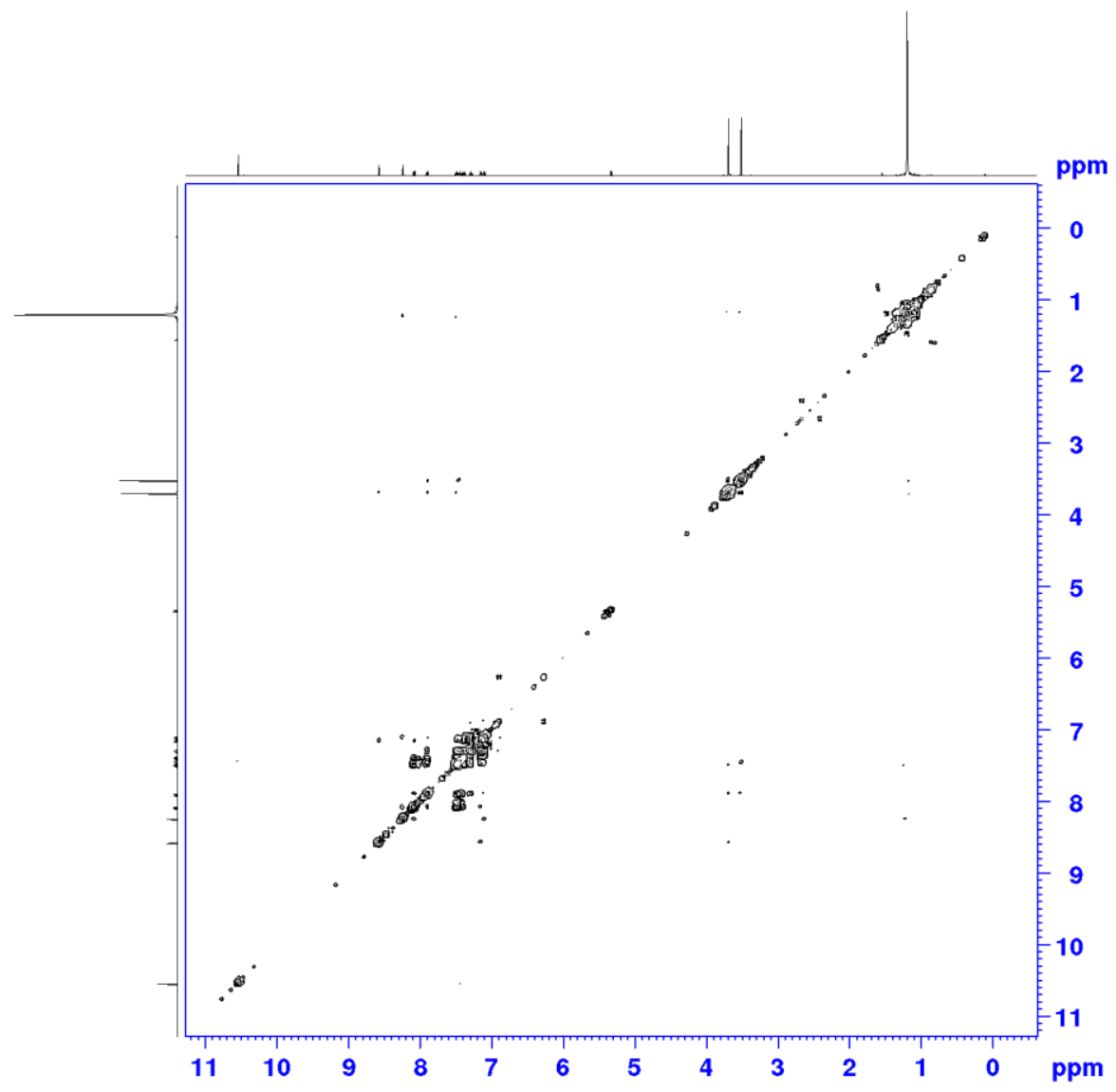

Figure S56. ${ }^{1} \mathrm{H},{ }^{1} \mathrm{H}$ gCOSY NMR spectrum of $\mathbf{1 5}(S)$ in $\mathrm{CDCl}_{3}$ at a $400 \mathrm{MHz}$ spectrometer at $298 \mathrm{~K}$. 


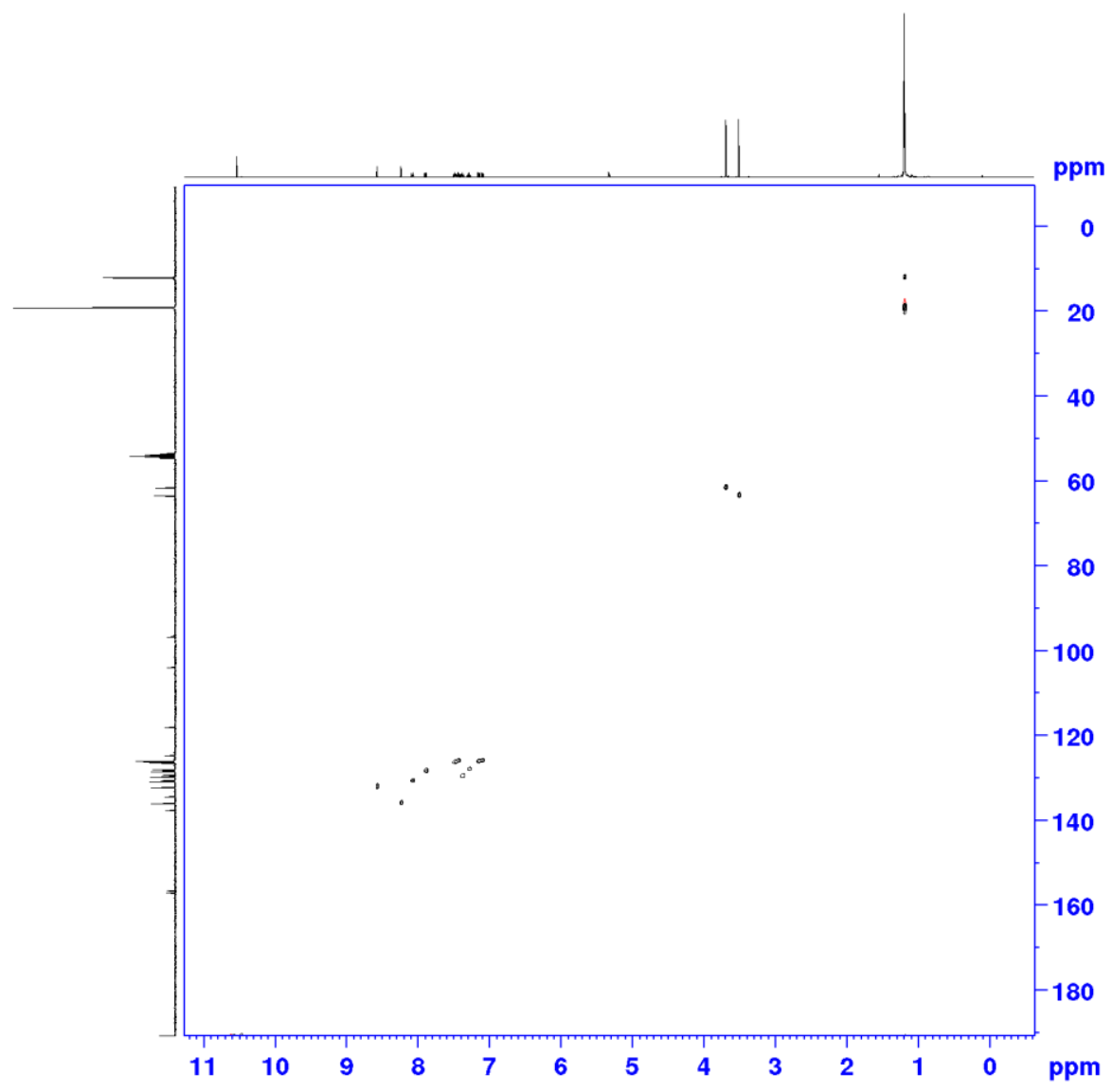

Figure S57. ${ }^{1} \mathrm{H},{ }^{13} \mathrm{C}$ gHSQC NMR spectrum of $\mathbf{1 5}(S)$ in $\mathrm{CDCl}_{3}$ at a $400 \mathrm{MHz}$ spectrometer at $298 \mathrm{~K}$.

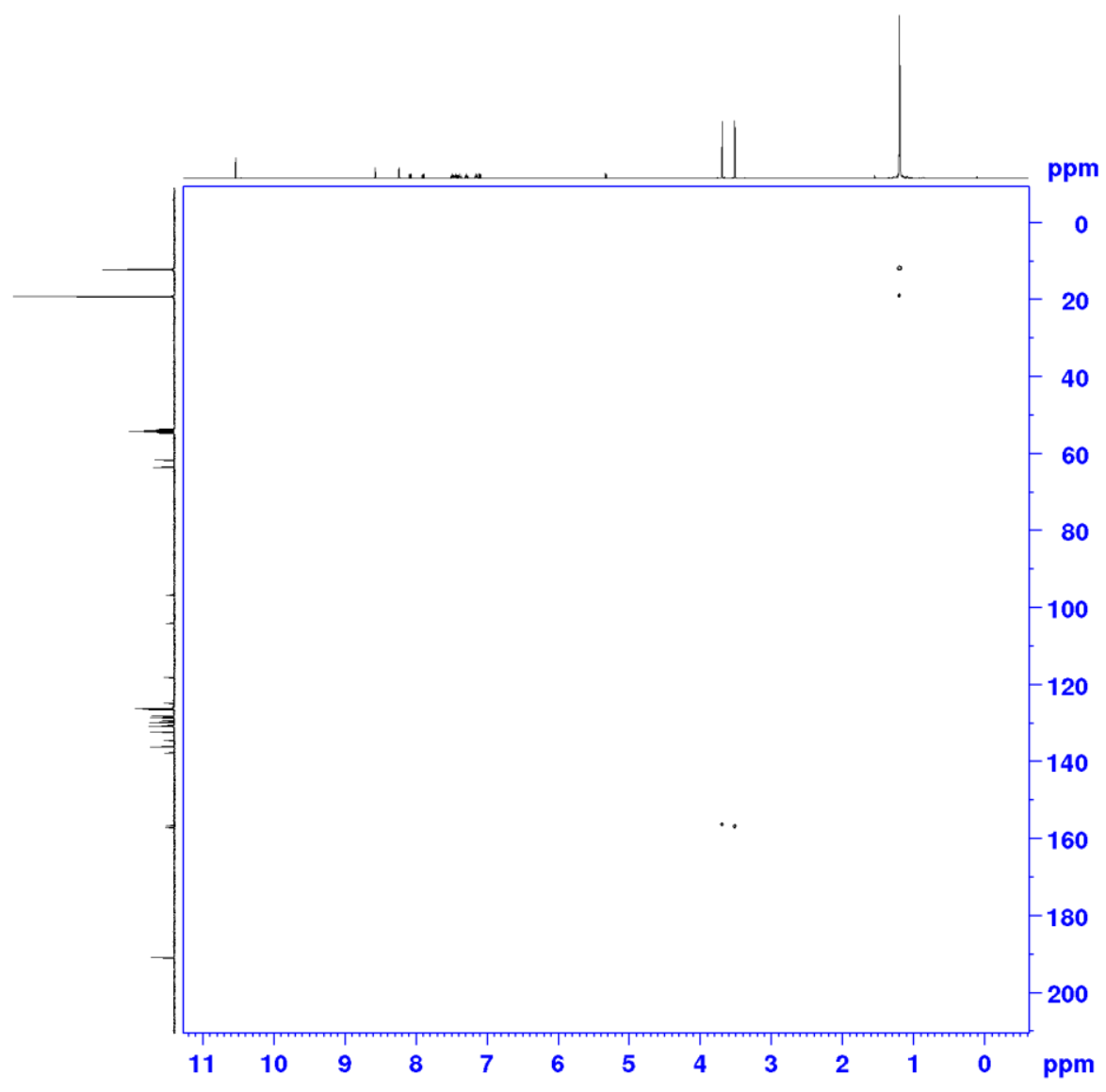

Figure S58. ${ }^{1} \mathrm{H},{ }^{13} \mathrm{C}$ gHMBC NMR spectrum of $\mathbf{1 5}(S)$ in $\mathrm{CDCl}_{3}$ at a $400 \mathrm{MHz}$ spectrometer at $298 \mathrm{~K}$. 


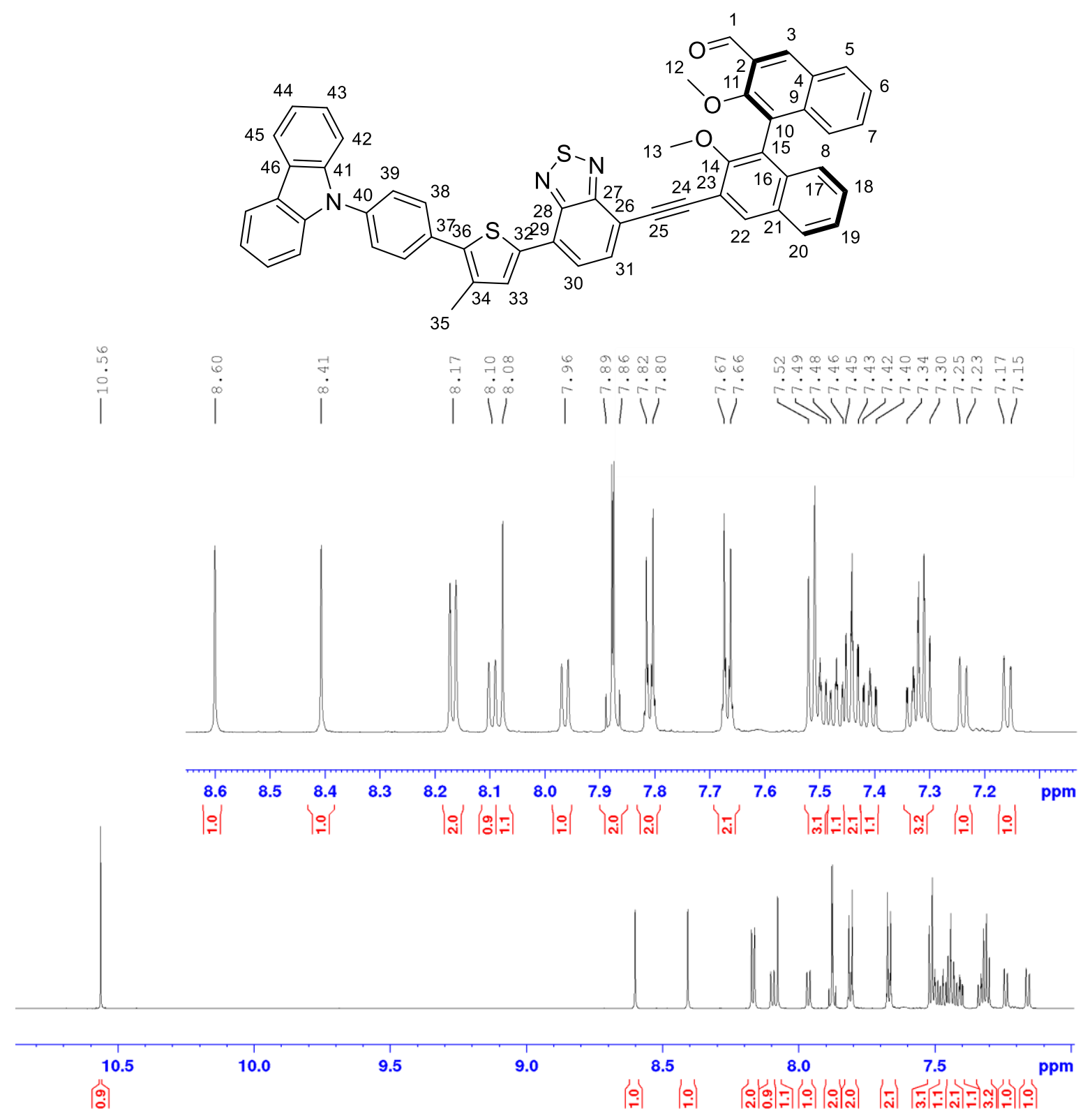

Figure S59. ${ }^{1} \mathrm{H}$ NMR of $\mathbf{1 7}(\boldsymbol{S})$ a in $\mathrm{CD}_{2} \mathrm{Cl}_{2}$ at a $700 \mathrm{MHz}$ spectrometer at $298 \mathrm{~K}$, Part 1 (10.7 ppm - 7.0 ppm). 


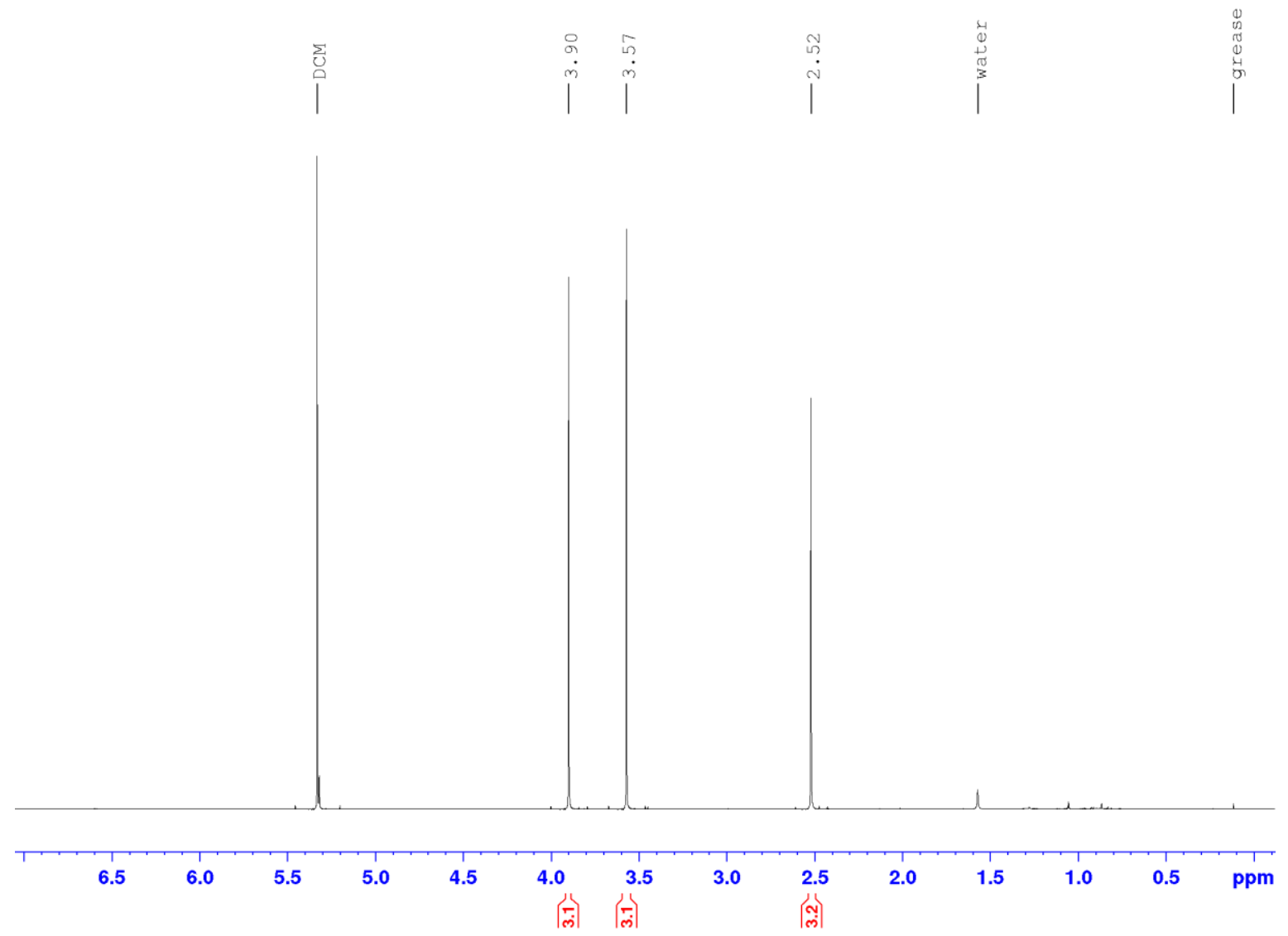

Figure S60. ${ }^{1} \mathrm{H}$ NMR of $\mathbf{1 7}(S)$ a in $\mathrm{CD}_{2} \mathrm{Cl}_{2}$ at a $700 \mathrm{MHz}$ spectrometer at $298 \mathrm{~K}$, Part 2 (7.0 ppm $-0.0 \mathrm{ppm})$.

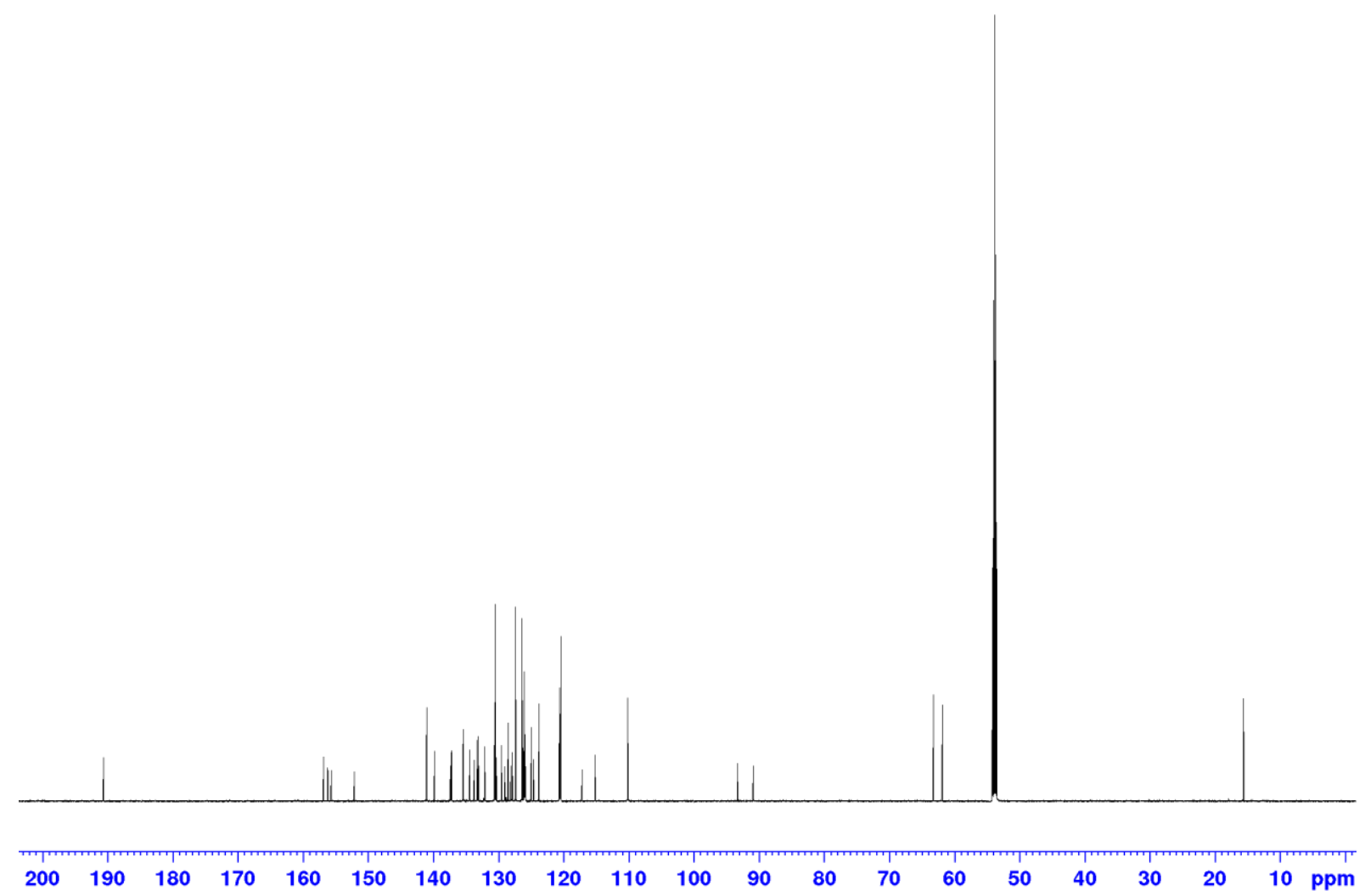

Figure S61. ${ }^{13} \mathrm{C}\left\{{ }^{1} \mathrm{H}\right\}$ NMR of $\mathbf{1 7}(S)$ a in $\mathrm{CD}_{2} \mathrm{Cl}_{2}$ at a $176.1 \mathrm{MHz}$ spectrometer at $298 \mathrm{~K}$, Overview. 


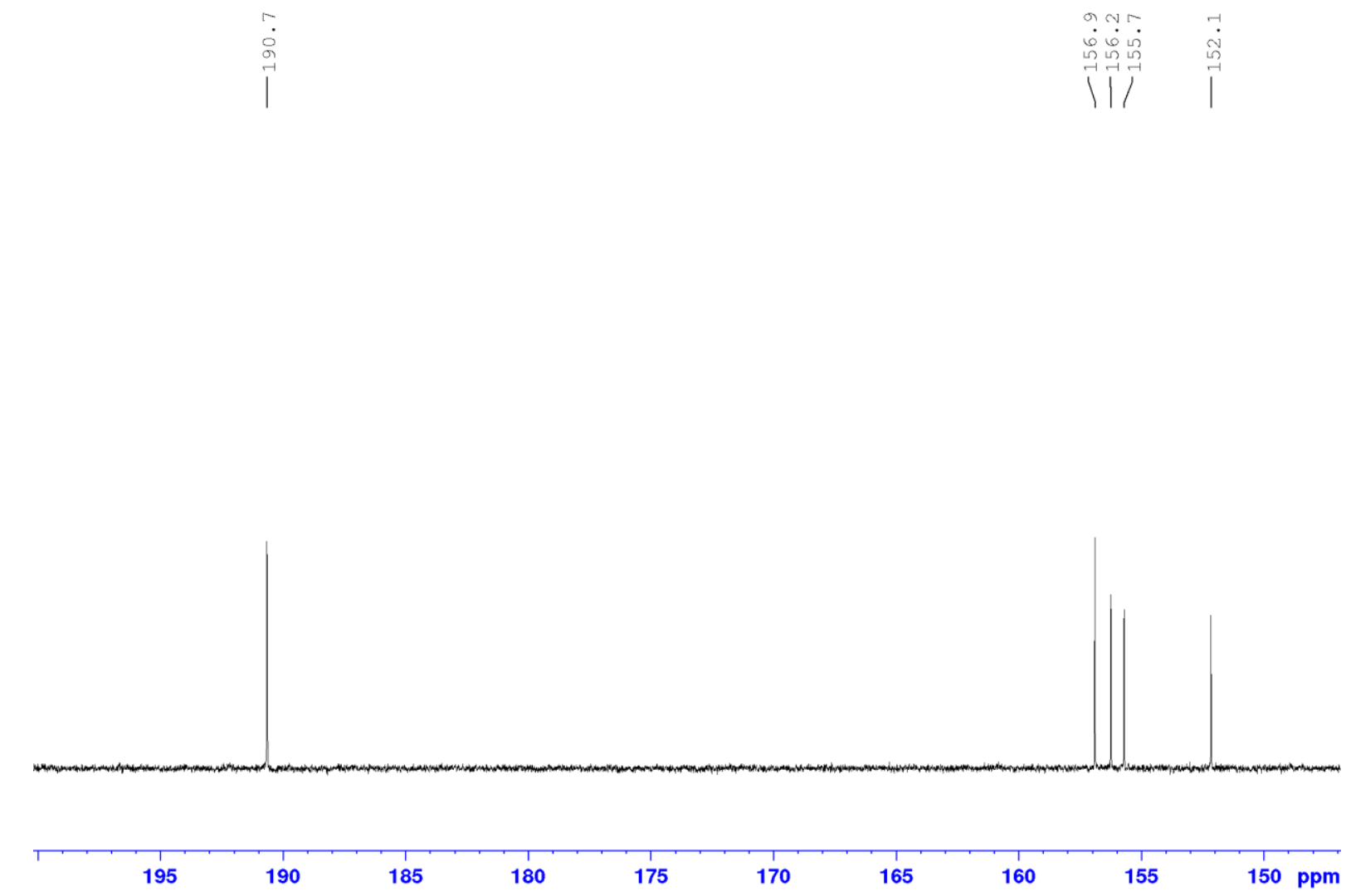

Figure S62. ${ }^{13} \mathrm{C}\left\{{ }^{1} \mathrm{H}\right\}$ NMR of $\mathbf{1 7}(\mathrm{S}) \mathbf{a}$ in $\mathrm{CD}_{2} \mathrm{Cl}_{2}$ at a $176.1 \mathrm{MHz}$ spectrometer at $298 \mathrm{~K}$, Part 1 (200 ppm - 147 ppm). 


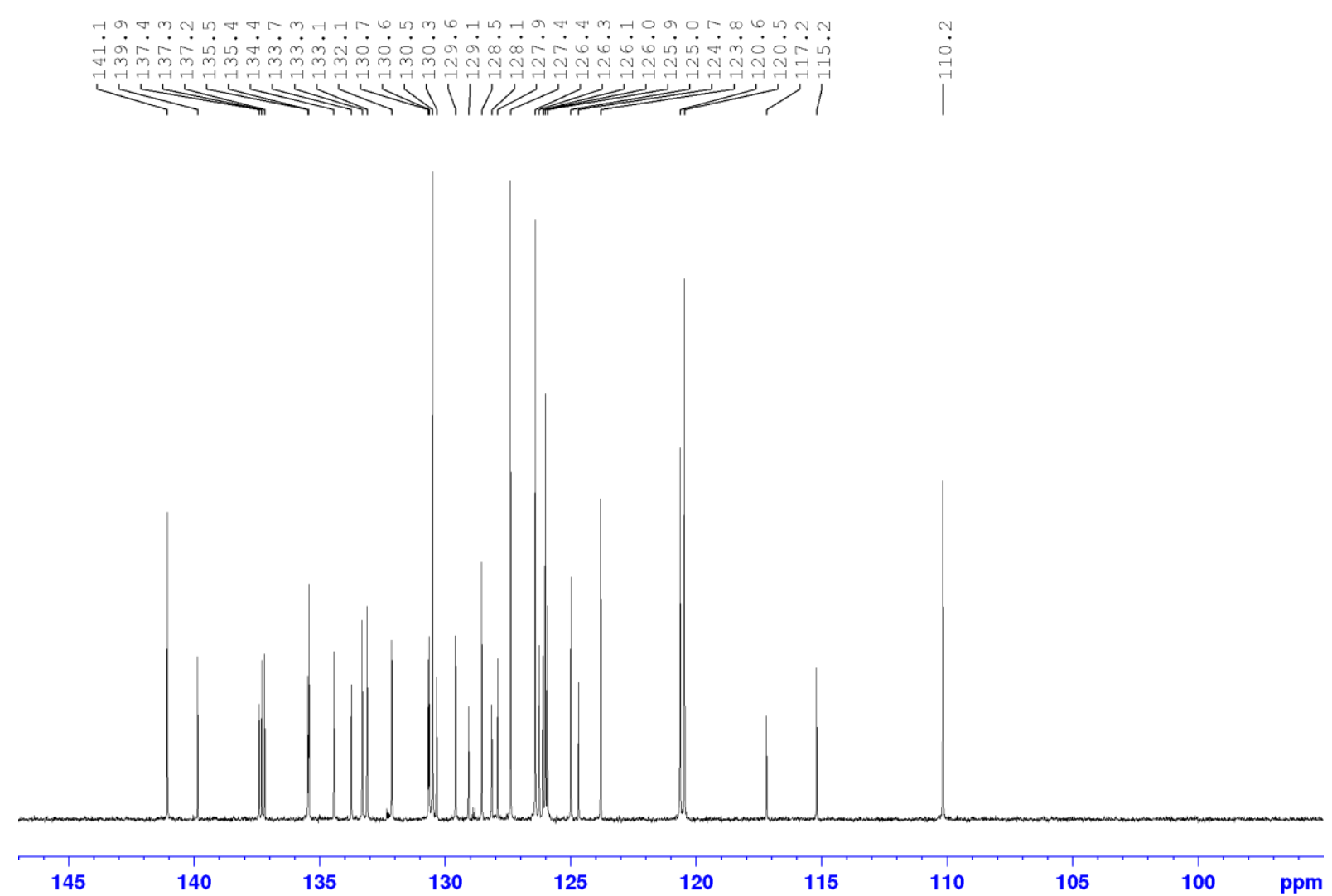

Figure S63. ${ }^{13} \mathrm{C}\left\{{ }^{1} \mathrm{H}\right\}$ NMR of $\mathbf{1 7}(S)$ a in $\mathrm{CD}_{2} \mathrm{Cl}_{2}$ at a $176.1 \mathrm{MHz}$ spectrometer at $298 \mathrm{~K}$, Part 2 (147 ppm-95 ppm).

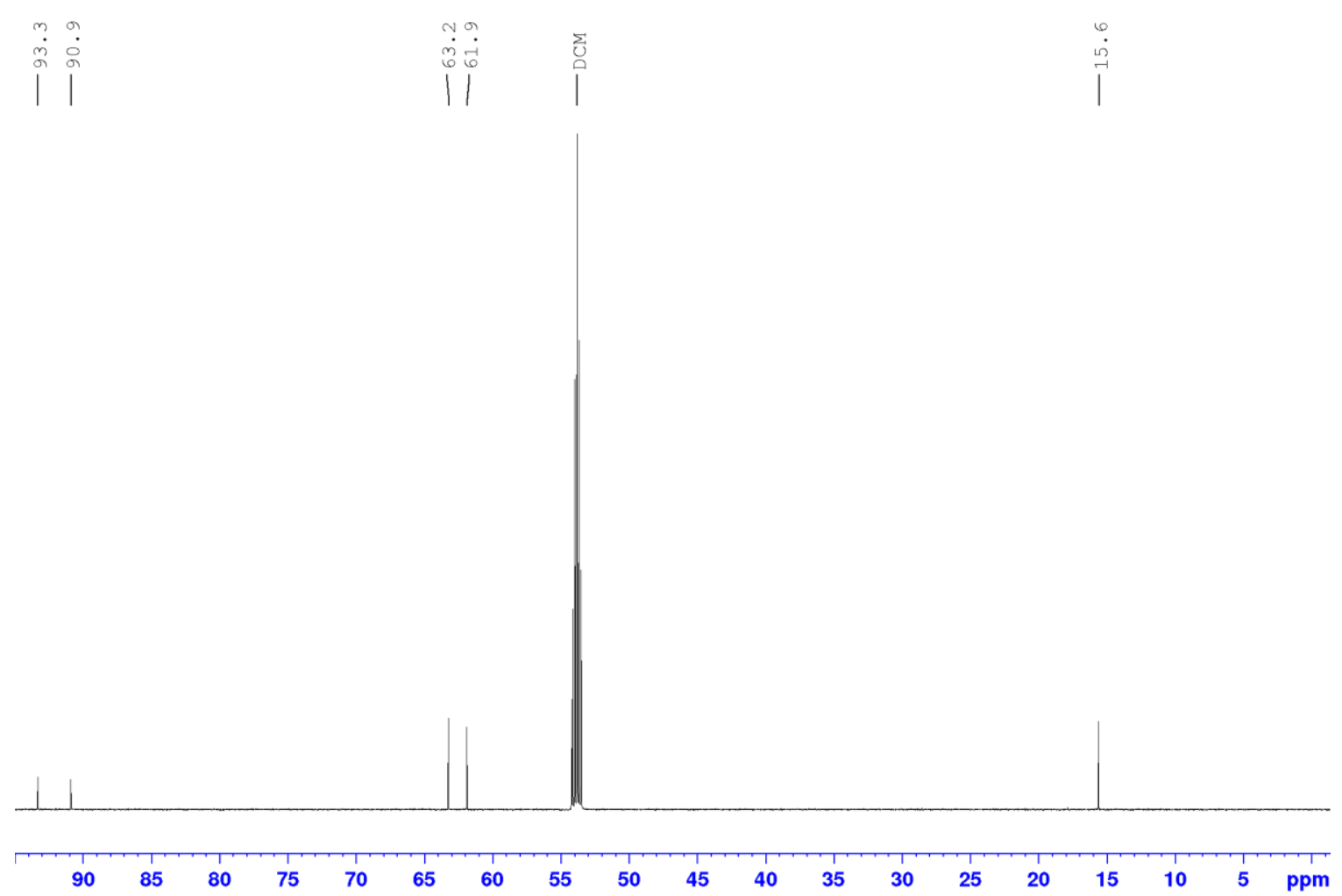

Figure S64. ${ }^{13} \mathrm{C}\left\{{ }^{1} \mathrm{H}\right\}$ NMR of $\mathbf{1 7}(\mathrm{S}) \mathbf{a}$ in $\mathrm{CD}_{2} \mathrm{Cl}_{2}$ at a $176.1 \mathrm{MHz}$ spectrometer at $298 \mathrm{~K}$, Part 3 (95 ppm-0 ppm). 


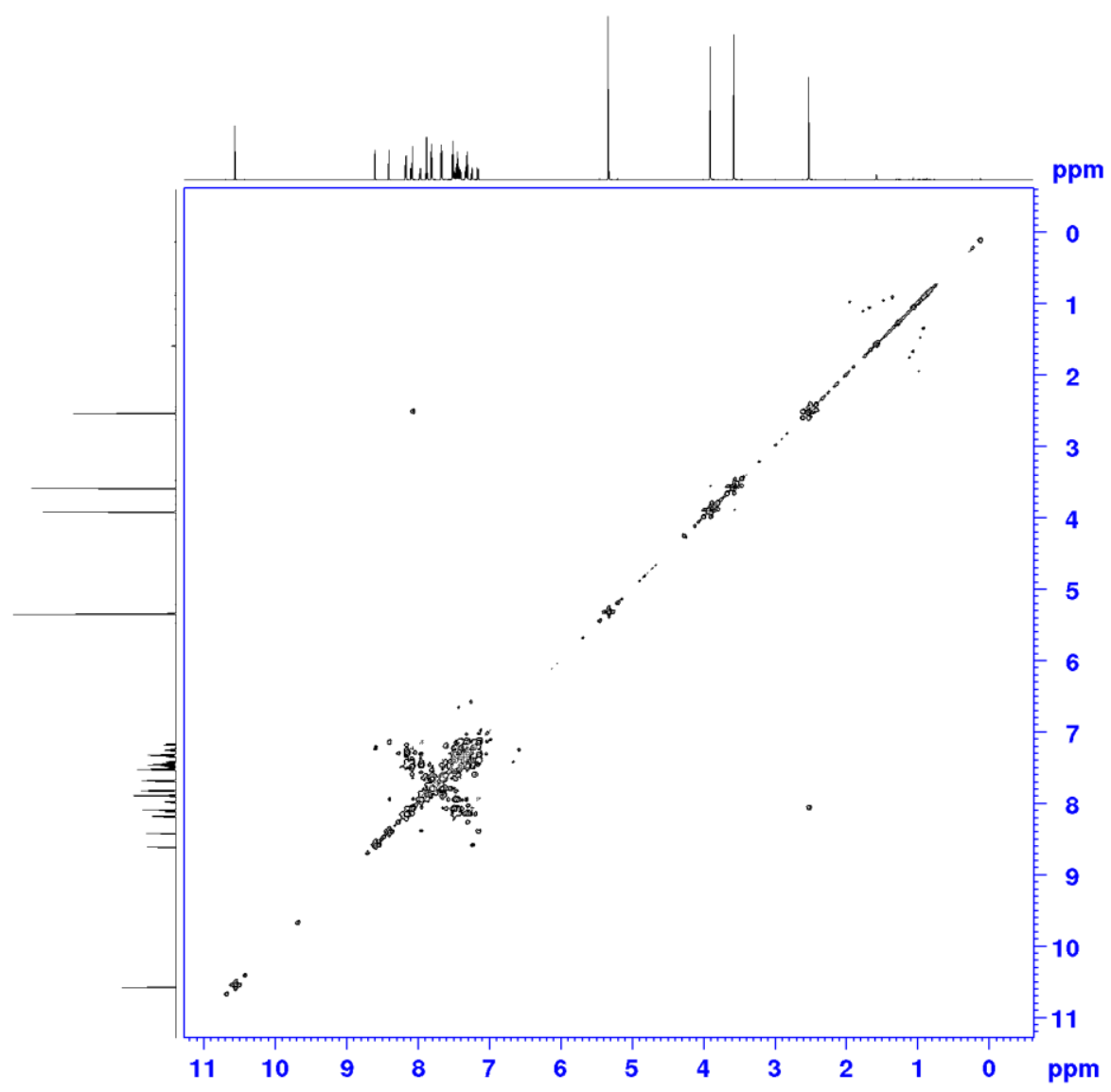

Figure S65. ${ }^{1} \mathrm{H},{ }^{1} \mathrm{H}$ gCOSY NMR spectrum of $\mathbf{1 7}(\boldsymbol{S}) \mathbf{a}$ in $\mathrm{CD}_{2} \mathrm{Cl}_{2}$ at a $700 \mathrm{MHz}$ spectrometer at $298 \mathrm{~K}$.

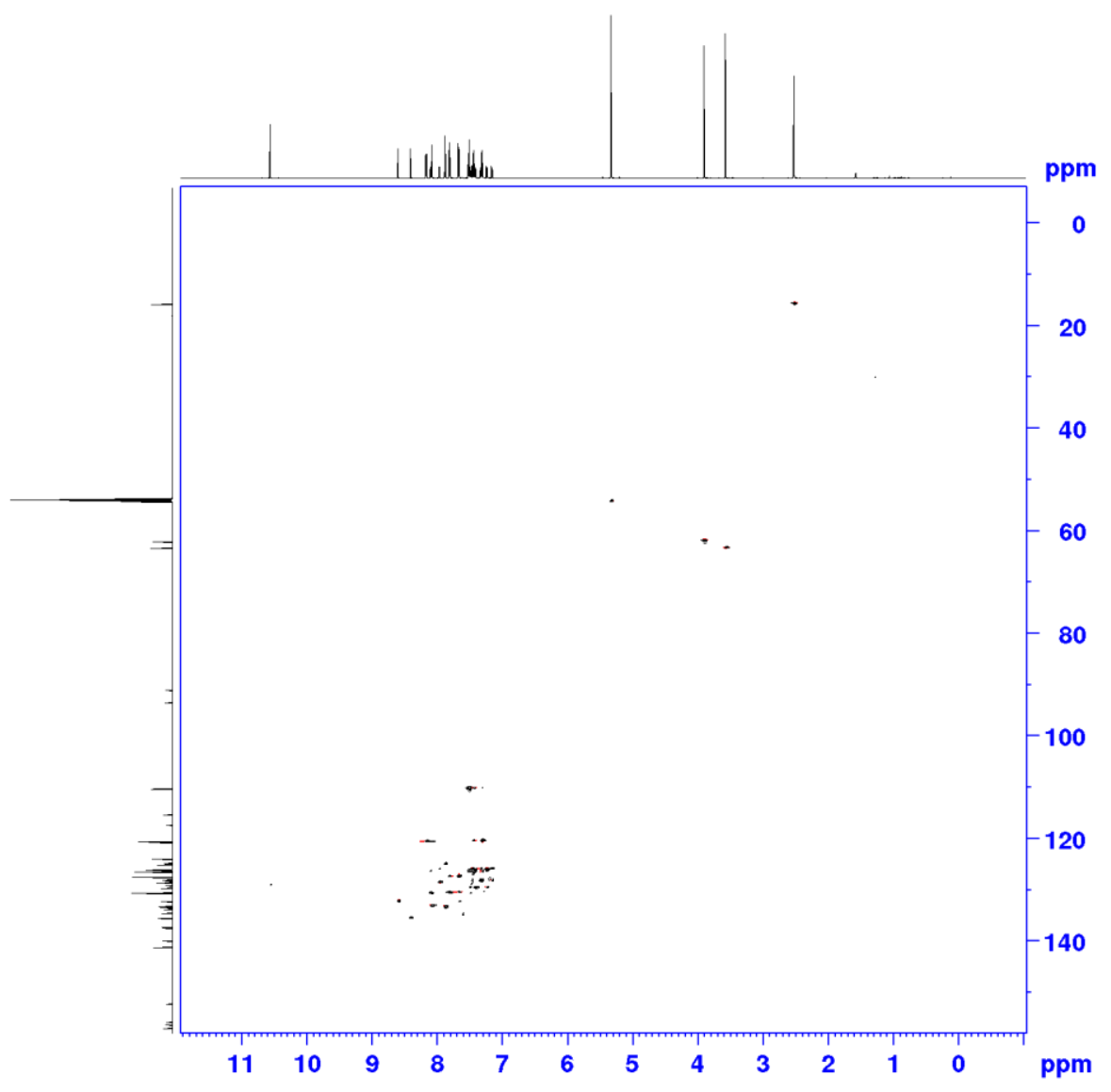

Figure S66. ${ }^{1} \mathrm{H},{ }^{13} \mathrm{C}$ gHSQC NMR spectrum of $\mathbf{1 7}(\boldsymbol{S})$ a in $\mathrm{CD}_{2} \mathrm{Cl}_{2}$ at a $700 \mathrm{MHz}$ spectrometer at $298 \mathrm{~K}$. 


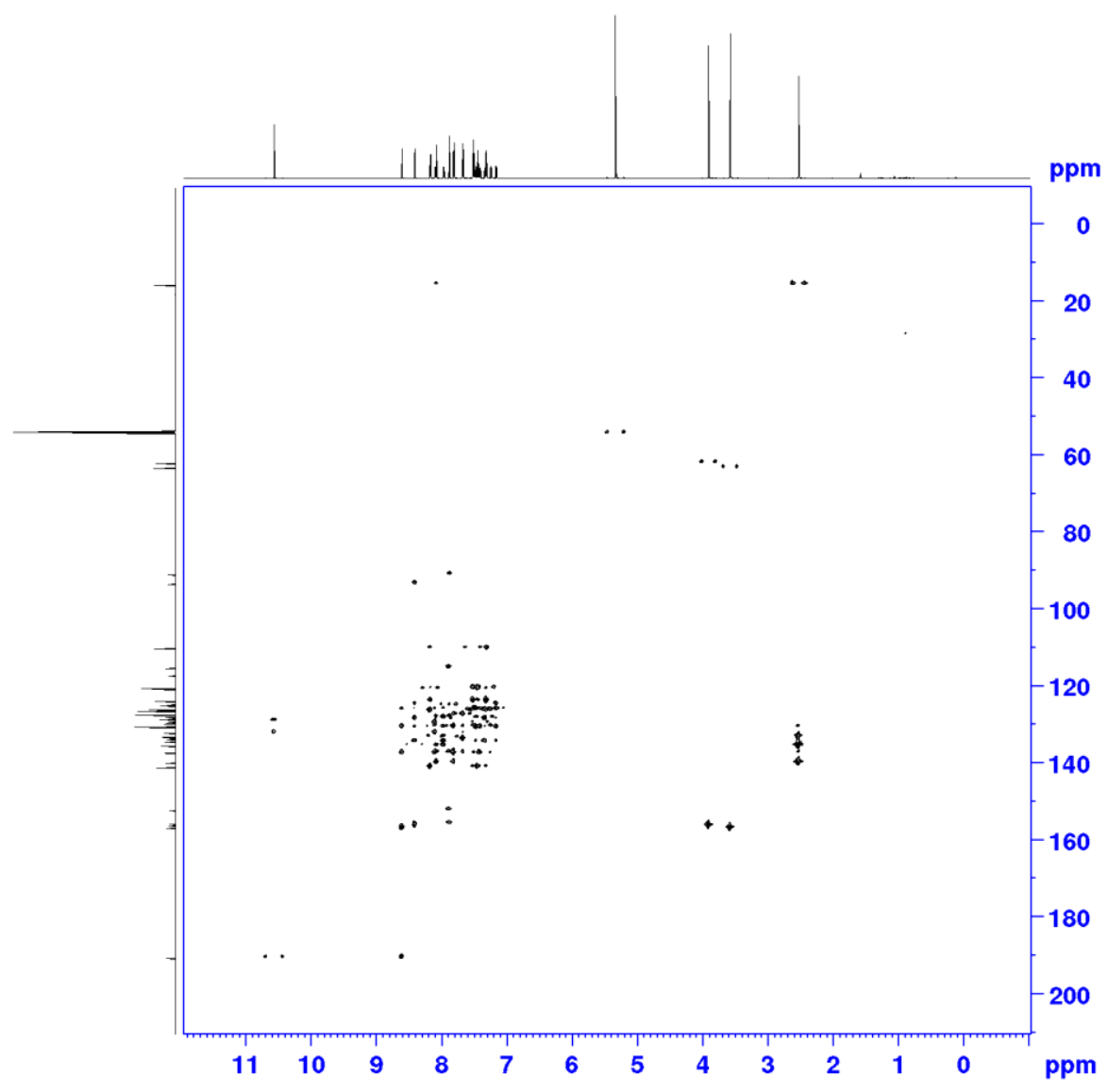

Figure S67. ${ }^{1} \mathrm{H},{ }^{13} \mathrm{C}$ gHMBC NMR spectrum of $\mathbf{1 7}(\mathrm{S}) \mathbf{a}$ in $\mathrm{CD}_{2} \mathrm{Cl}_{2}$ at a $700 \mathrm{MHz}$ spectrometer at $298 \mathrm{~K}$. 


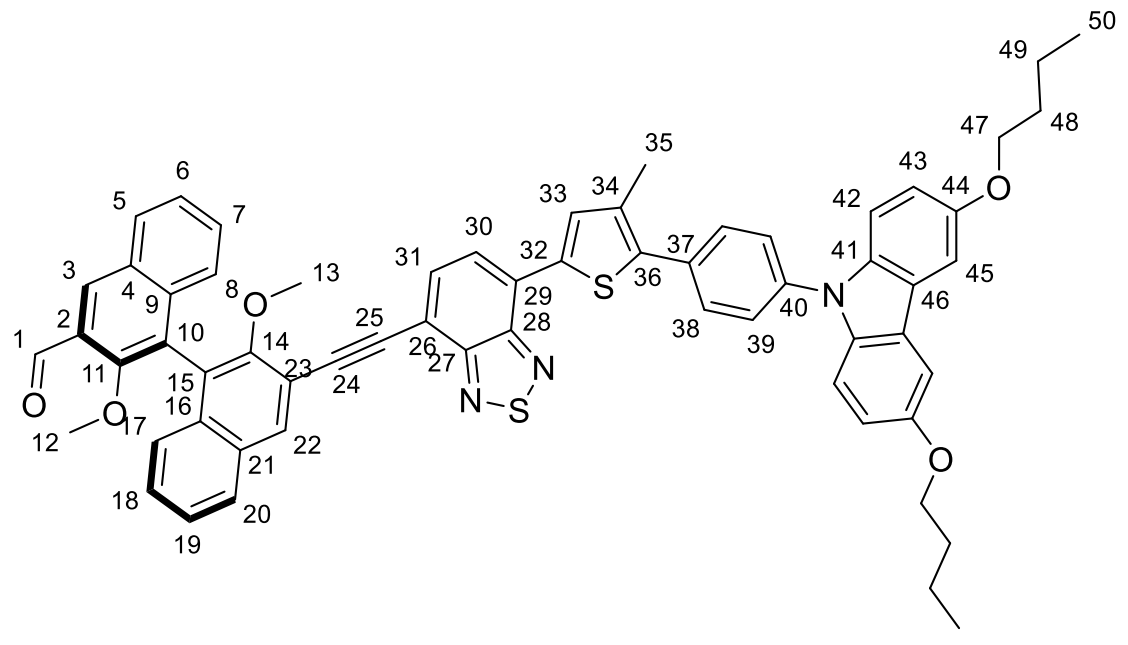

|
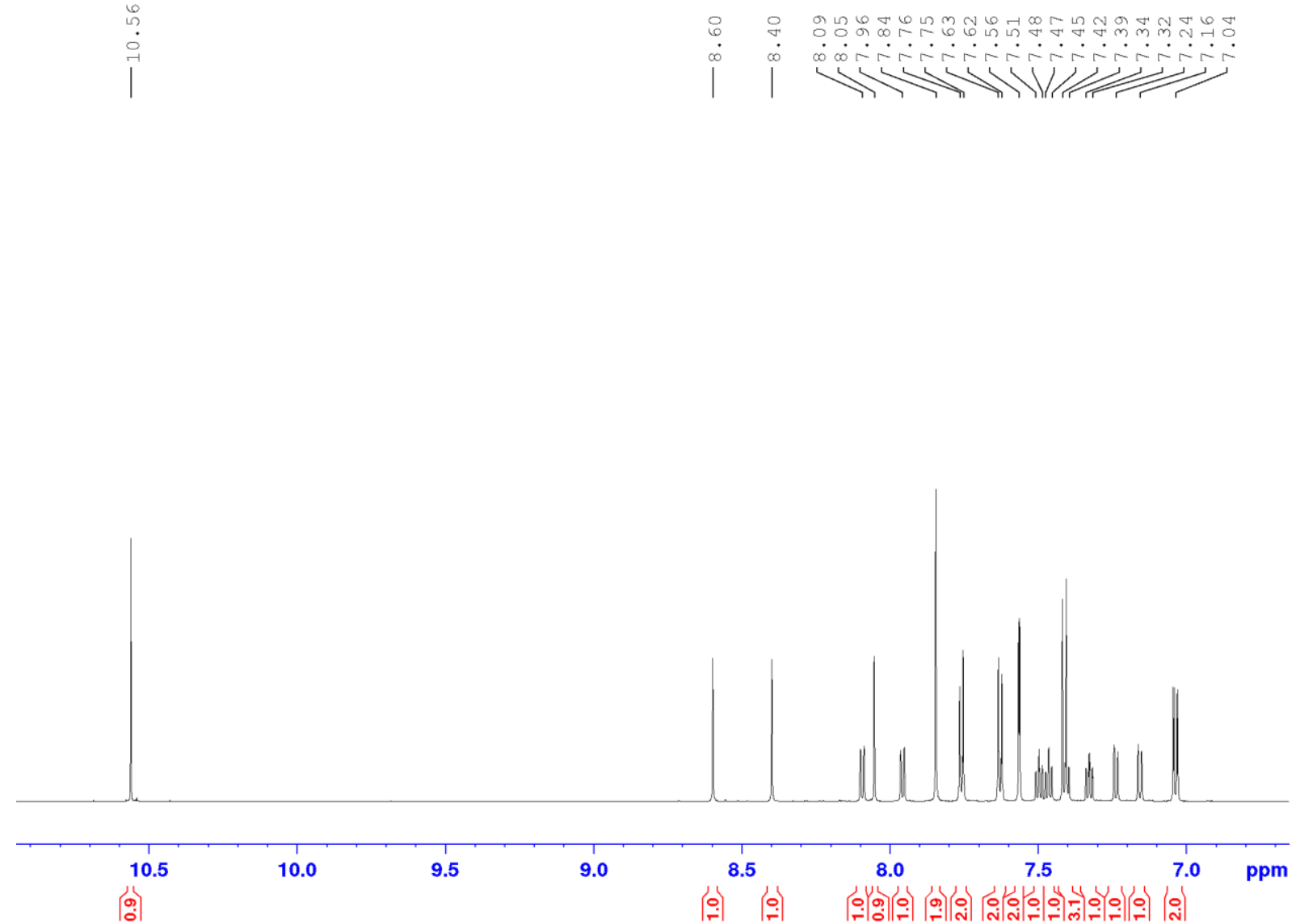

Figure S68. ${ }^{1} \mathrm{H}$ NMR of $\mathbf{1 7}(S)$ b in $\mathrm{CD}_{2} \mathrm{Cl}_{2}$ at a $700 \mathrm{MHz}$ spectrometer at $298 \mathrm{~K}$, Part 1 (10.9 ppm - 6.7 ppm). 


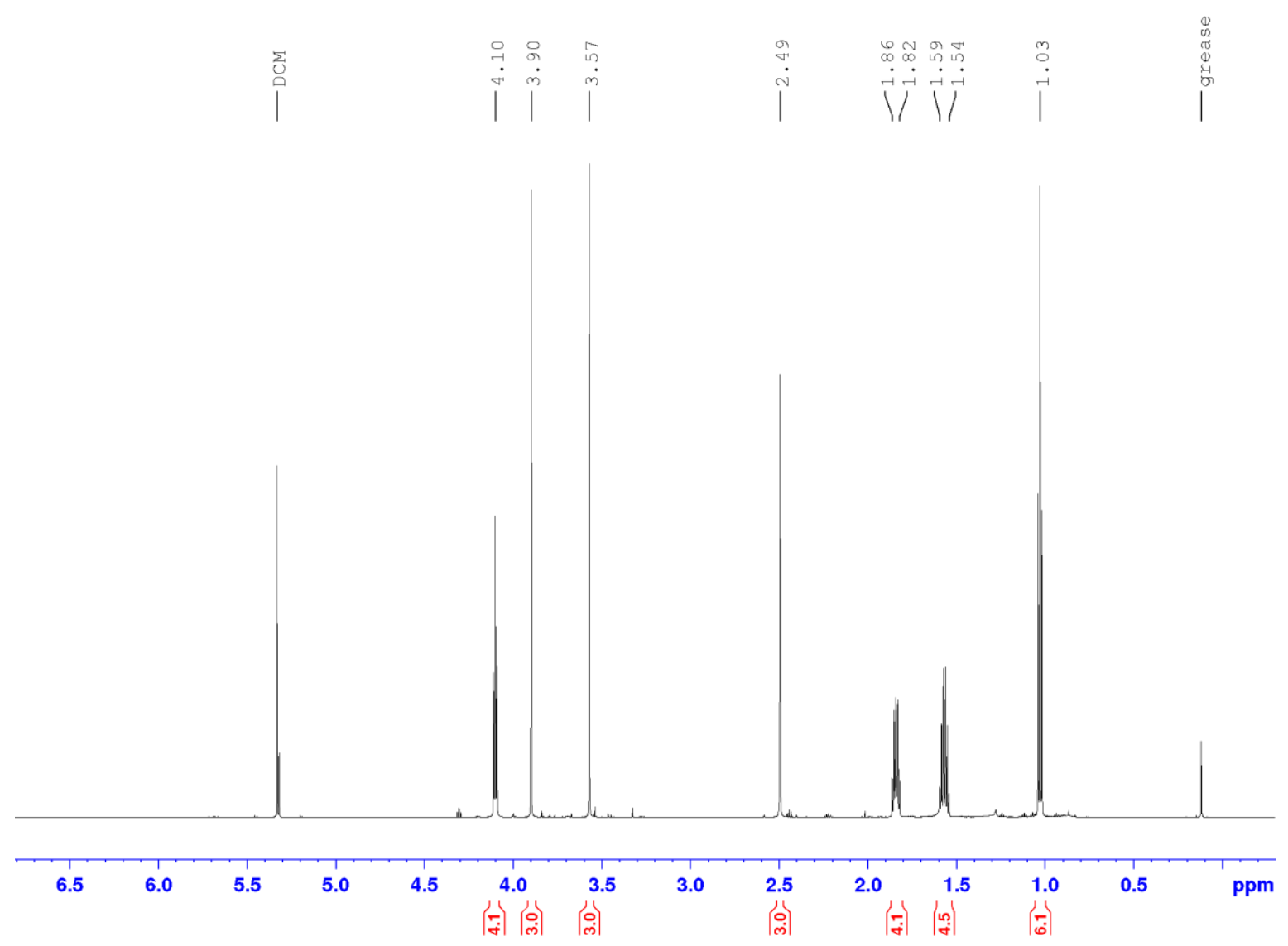

Figure S69. ${ }^{1} \mathrm{H}$ NMR of $\mathbf{1 7}(S)$ b in $\mathrm{CD}_{2} \mathrm{Cl}_{2}$ at a $700 \mathrm{MHz}$ spectrometer at $298 \mathrm{~K}$, Part 2 $(6.8 \mathrm{ppm}-0.0 \mathrm{ppm})$.

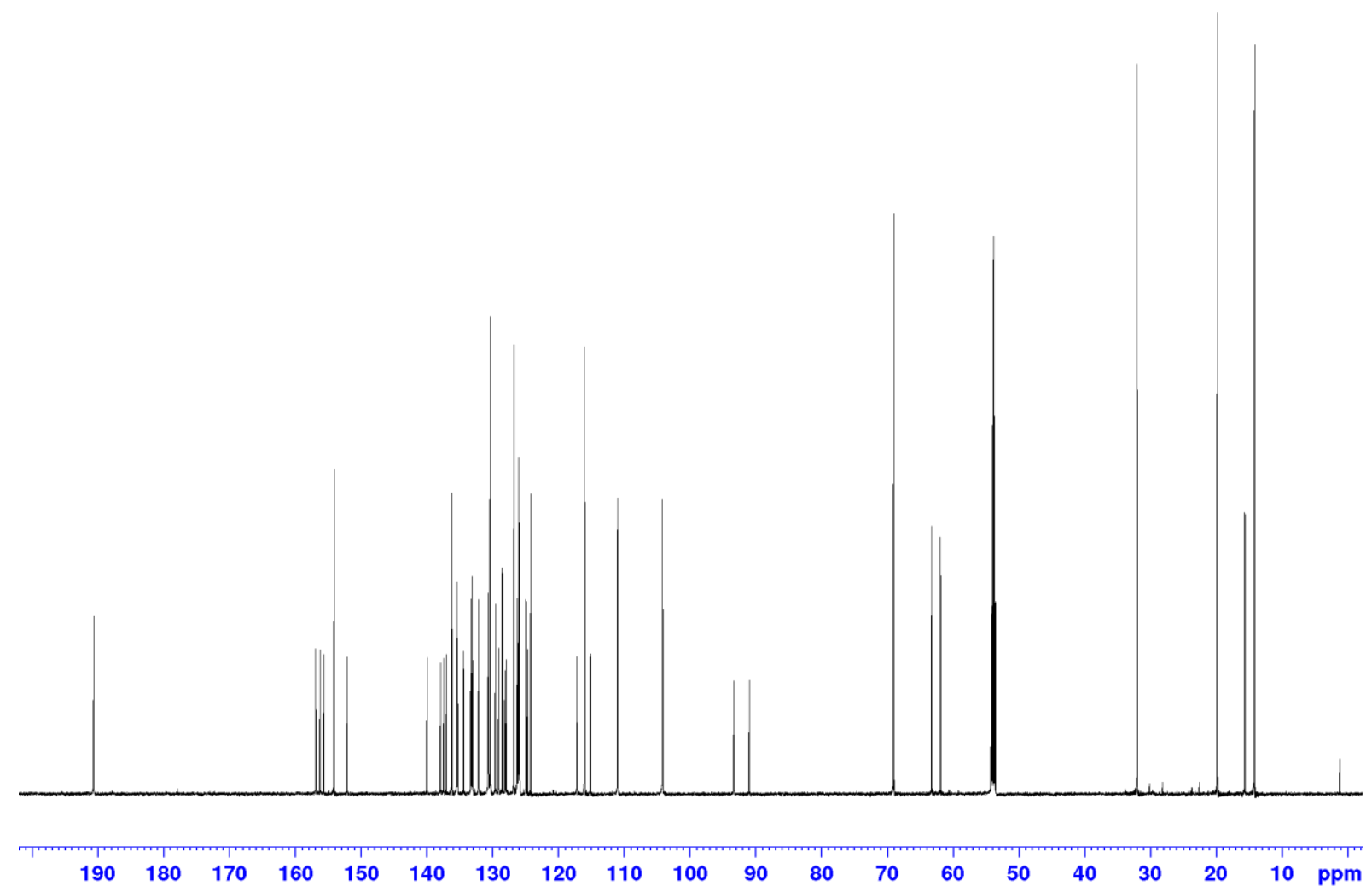

Figure S70. ${ }^{13} \mathrm{C}\left\{{ }^{1} \mathrm{H}\right\}$ NMR of $\mathbf{1 7}(S)$ b in $\mathrm{CD}_{2} \mathrm{Cl}_{2}$ at a $176.1 \mathrm{MHz}$ spectrometer at $298 \mathrm{~K}$, Overview. 


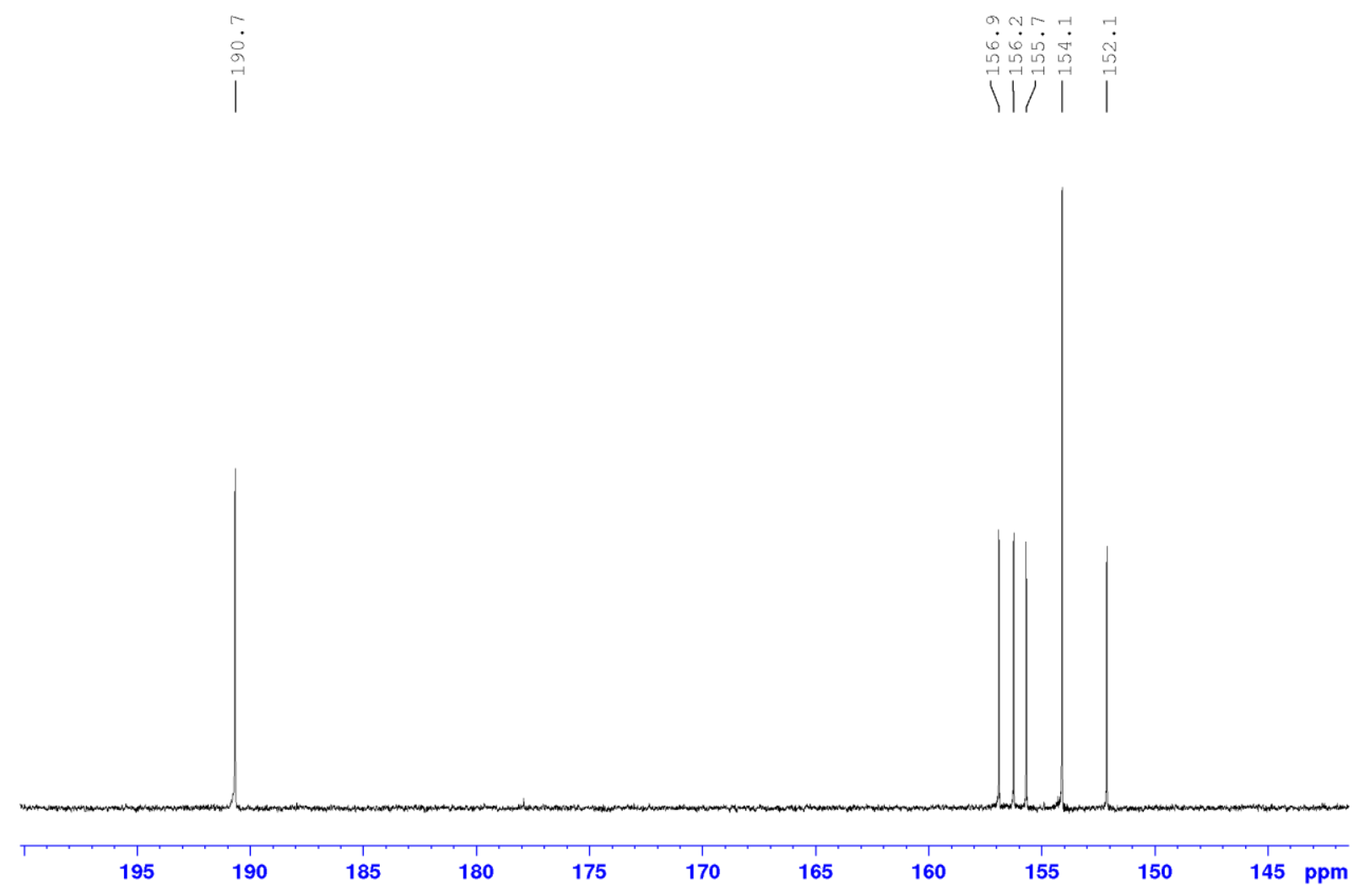

Figure S71. ${ }^{13} \mathrm{C}\left\{{ }^{1} \mathrm{H}\right\}$ NMR of $\mathbf{1 7}(S)$ b in $\mathrm{CD}_{2} \mathrm{Cl}_{2}$ at a $176.1 \mathrm{MHz}$ spectrometer at $298 \mathrm{~K}$, Part 1 (200 ppm - 143 ppm). 


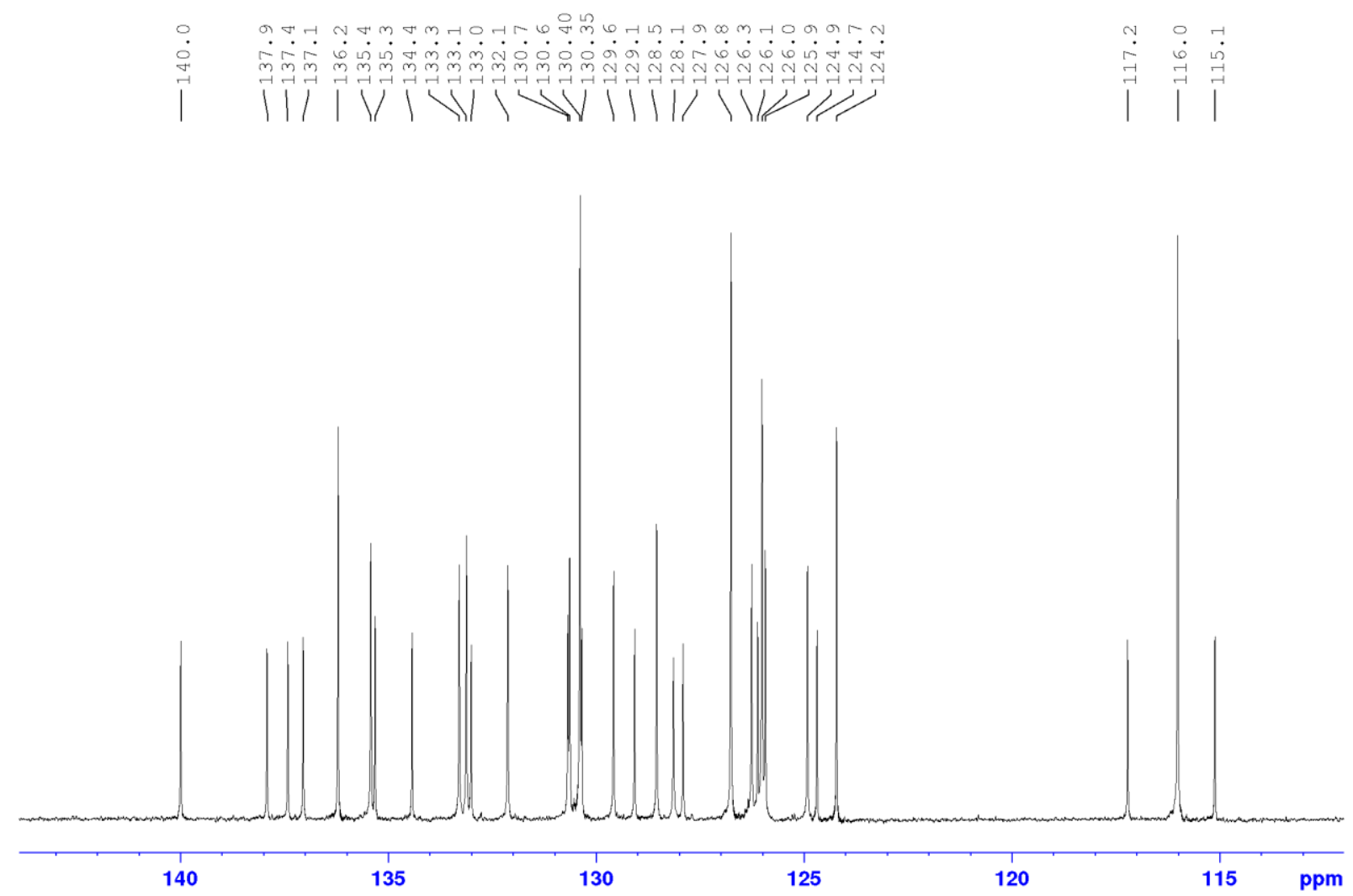

Figure S72. ${ }^{13} \mathrm{C}\left\{{ }^{1} \mathrm{H}\right\}$ NMR of $\mathbf{1 7}(S)$ b in $\mathrm{CD}_{2} \mathrm{Cl}_{2}$ at a $176.1 \mathrm{MHz}$ spectrometer at $298 \mathrm{~K}$, Part 2 (143 ppm-113 ppm).

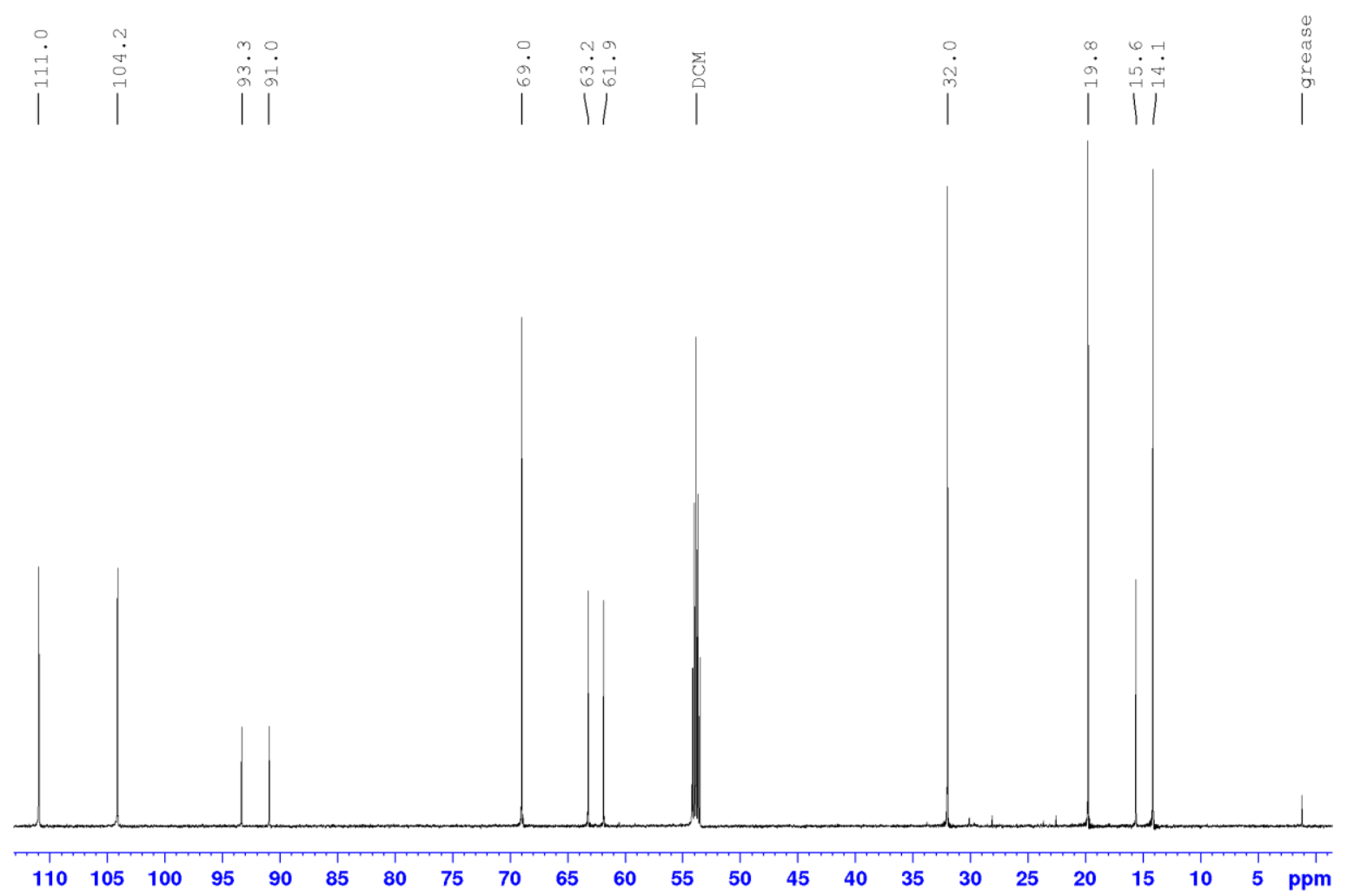

Figure S73. ${ }^{13} \mathrm{C}\left\{{ }^{1} \mathrm{H}\right\}$ NMR of $\mathbf{1 7}(S)$ b in $\mathrm{CD}_{2} \mathrm{Cl}_{2}$ at a $176.1 \mathrm{MHz}$ spectrometer at $298 \mathrm{~K}$, Part 3 (113 ppm-0 ppm). 


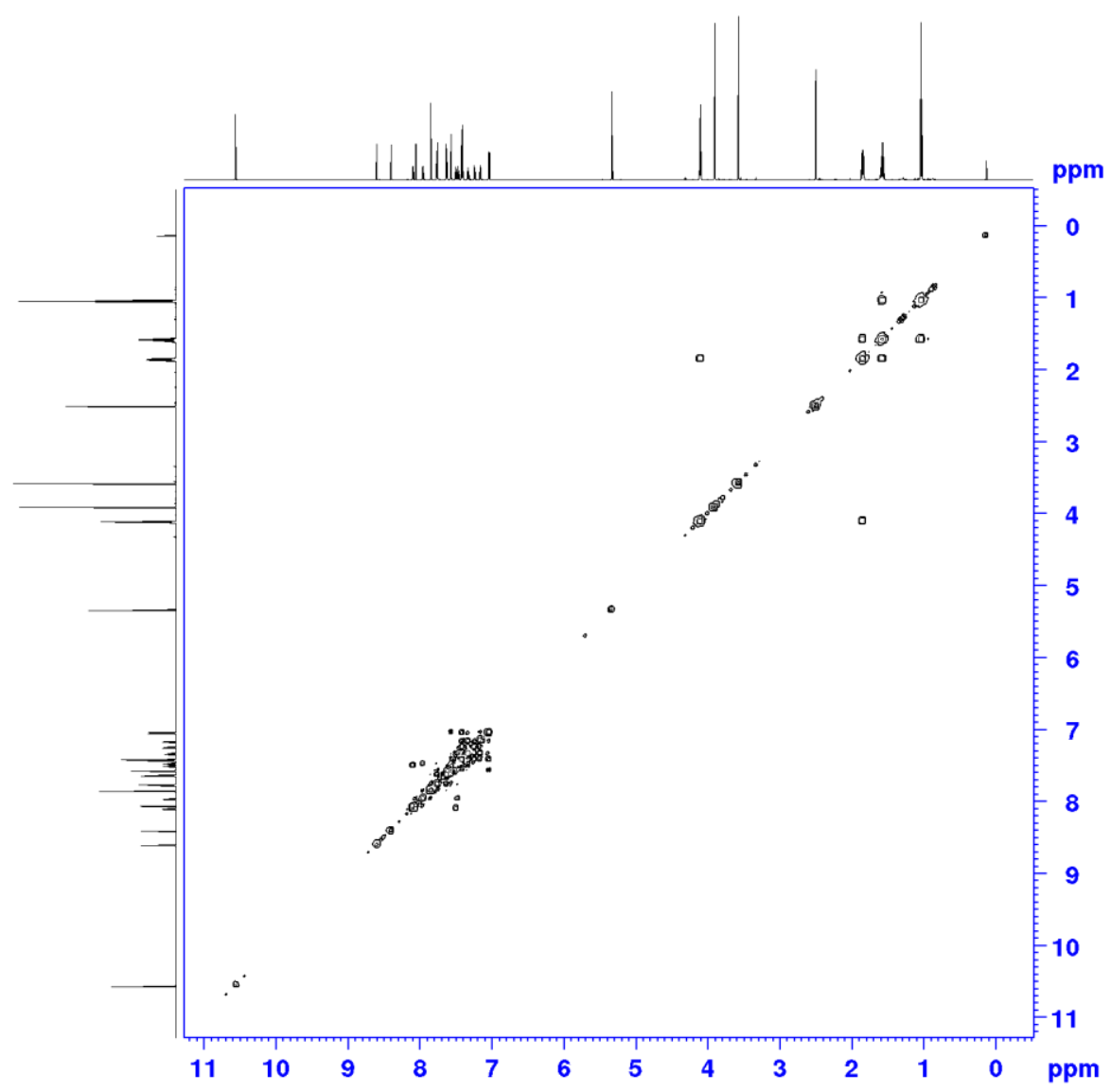

Figure S74. ${ }^{1} \mathrm{H},{ }^{1} \mathrm{H}$ gCOSY NMR spectrum of $\mathbf{1 7}(S)$ b in $\mathrm{CD}_{2} \mathrm{Cl}_{2}$ at a $700 \mathrm{MHz}$ spectrometer at $298 \mathrm{~K}$.

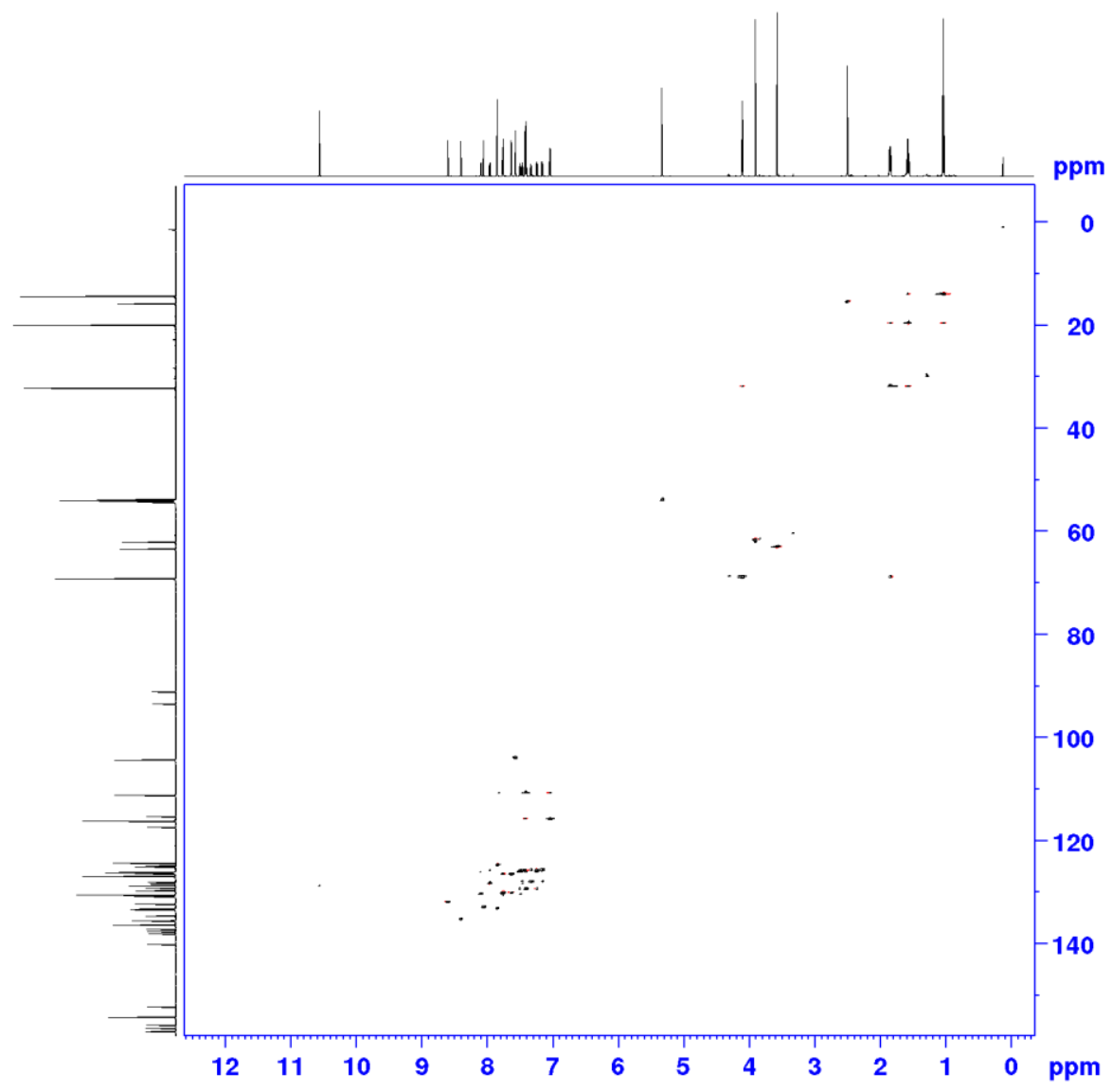

Figure S75. ${ }^{1} \mathrm{H},{ }^{13} \mathrm{C}$ gHSQC NMR spectrum of $\mathbf{1 7}(\mathrm{S}) \mathbf{b}$ in $\mathrm{CD}_{2} \mathrm{Cl}_{2}$ at a $700 \mathrm{MHz}$ spectrometer at $298 \mathrm{~K}$. 


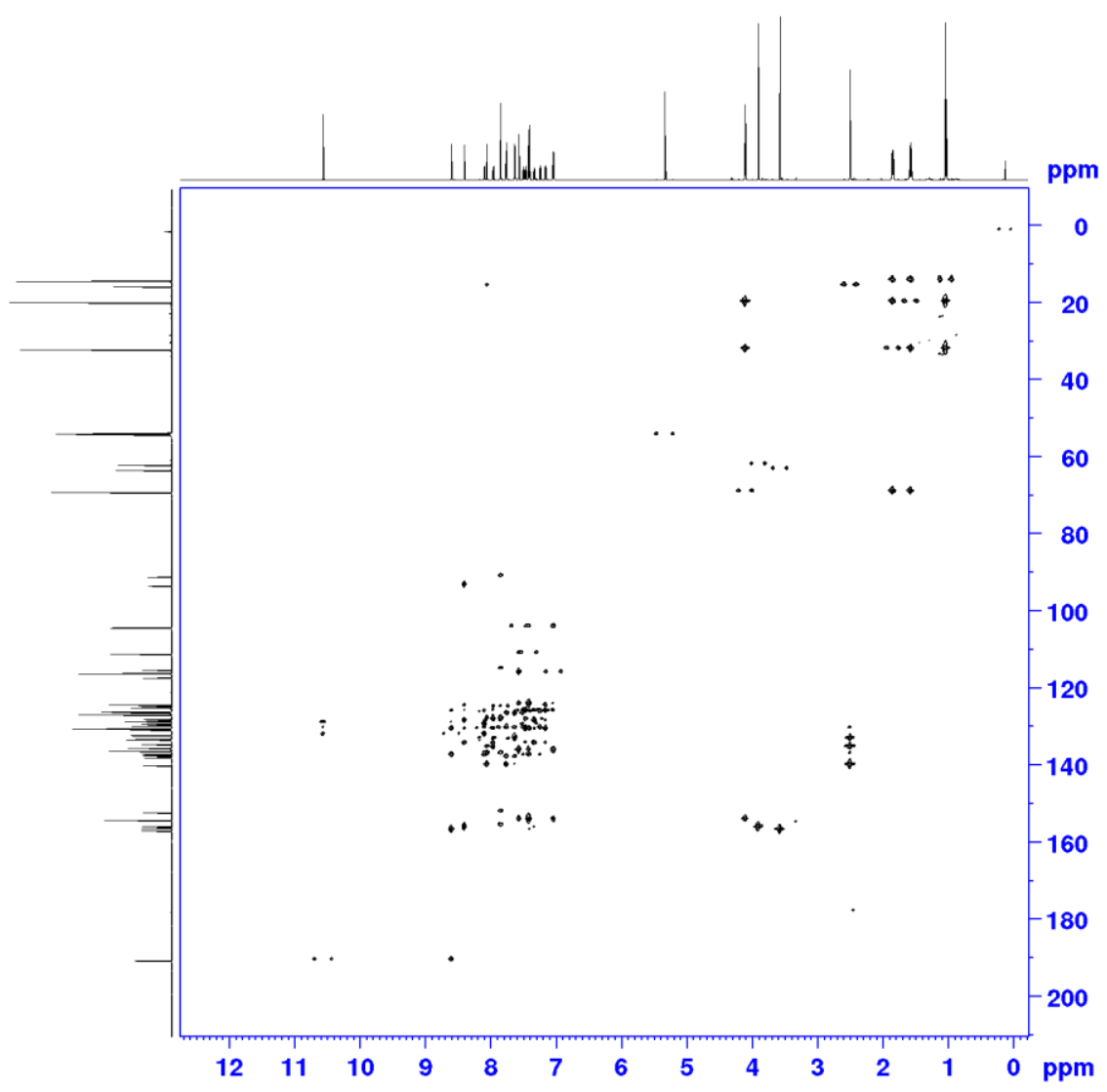

Figure S76. ${ }^{1} \mathrm{H},{ }^{13} \mathrm{C}$ gHMBC NMR spectrum of $\mathbf{1 7}(\mathrm{S})$ b in $\mathrm{CD}_{2} \mathrm{Cl}_{2}$ at a $700 \mathrm{MHz}$ spectrometer at $298 \mathrm{~K}$.

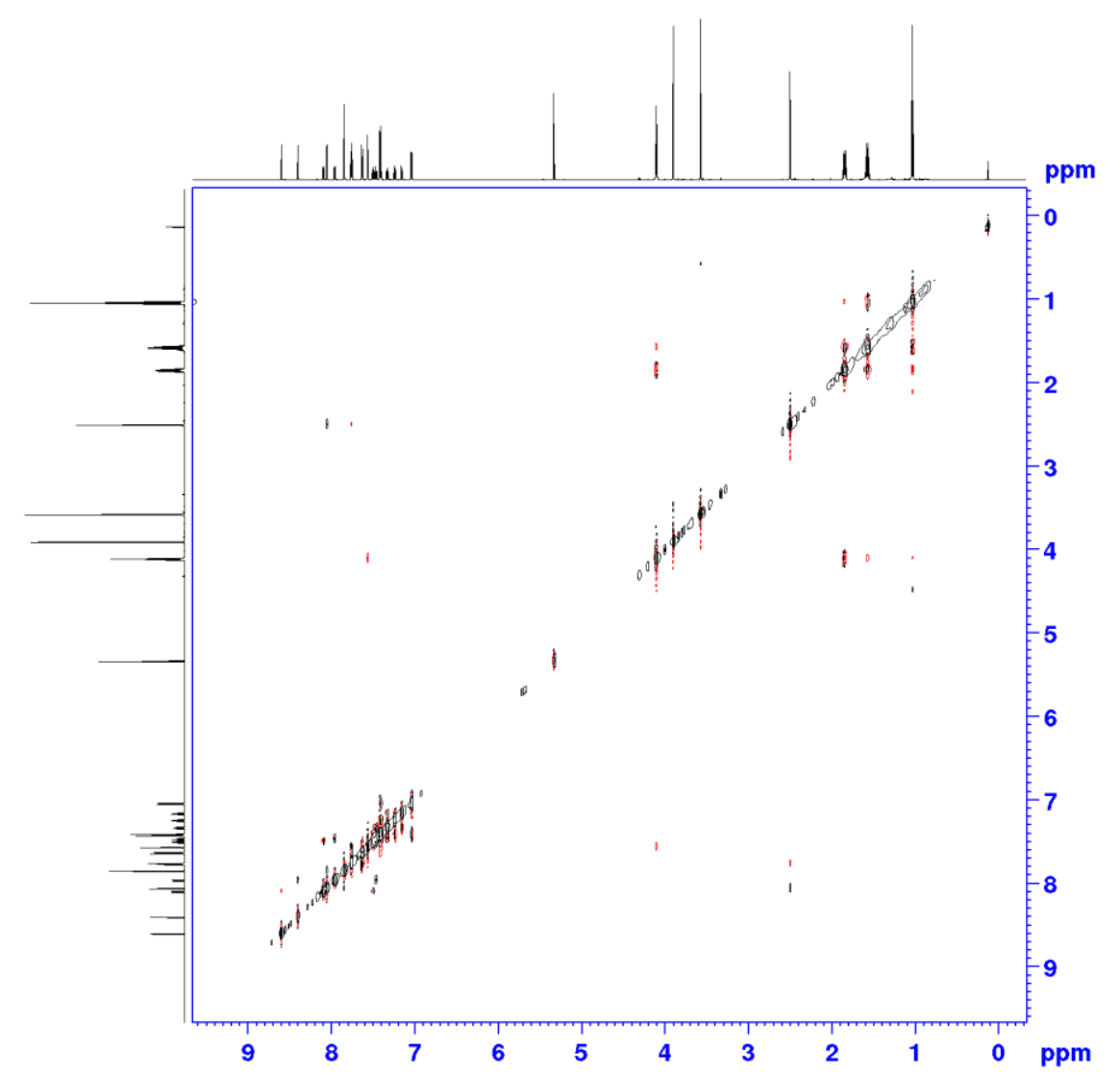

Figure S77. ${ }^{1} \mathrm{H},{ }^{1} \mathrm{H}$ gNOESY NMR spectrum of $\mathbf{1 7}(S)$ b in $\mathrm{CD}_{2} \mathrm{Cl}_{2}$ at a $700 \mathrm{MHz}$ spectrometer at $298 \mathrm{~K}$. 

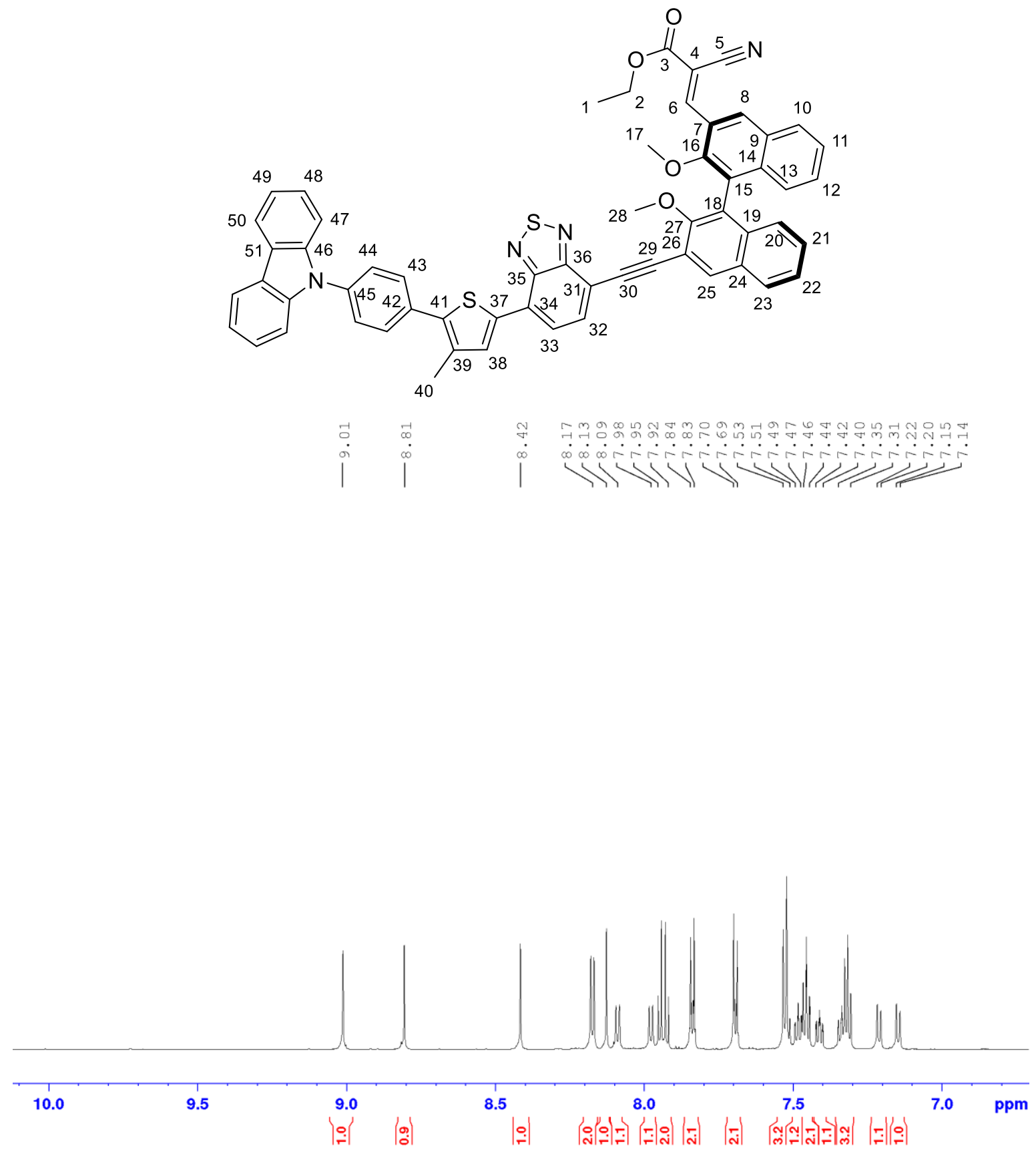

Figure S78. ${ }^{1} \mathrm{H} \mathrm{NMR}$ of $\mathbf{1 8}(S)$ a in $\mathrm{CD}_{2} \mathrm{Cl}_{2}$ at a $700 \mathrm{MHz}$ spectrometer at $298 \mathrm{~K}$, Part 1 (10.1 ppm-6.8 ppm). 


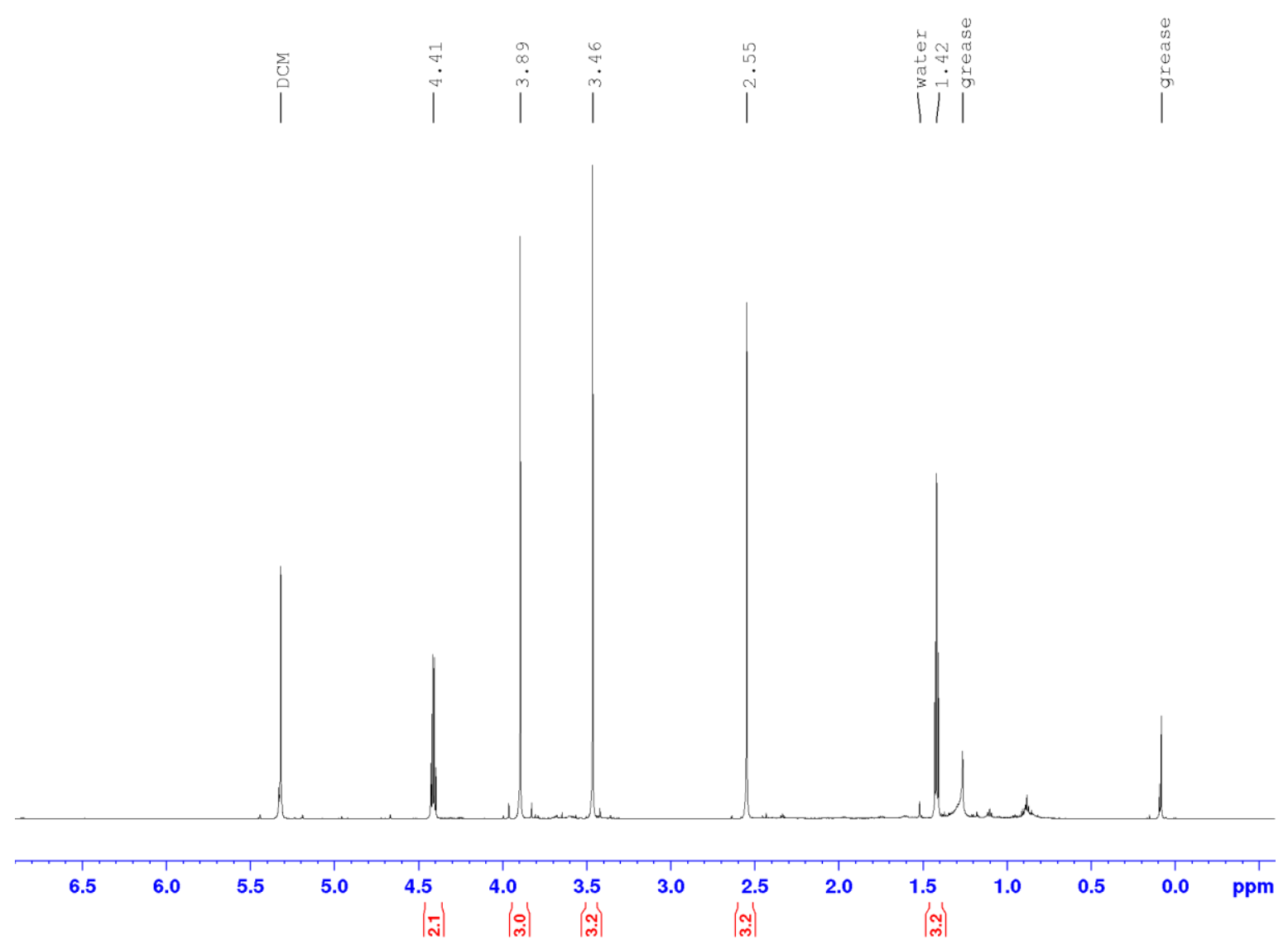

Figure S79. ${ }^{1} \mathrm{H}$ NMR of $\mathbf{1 8}(S)$ a in $\mathrm{CD}_{2} \mathrm{Cl}_{2}$ at a $700 \mathrm{MHz}$ spectrometer at $298 \mathrm{~K}$, Part 2 (6.8 ppm - -1.0 ppm).

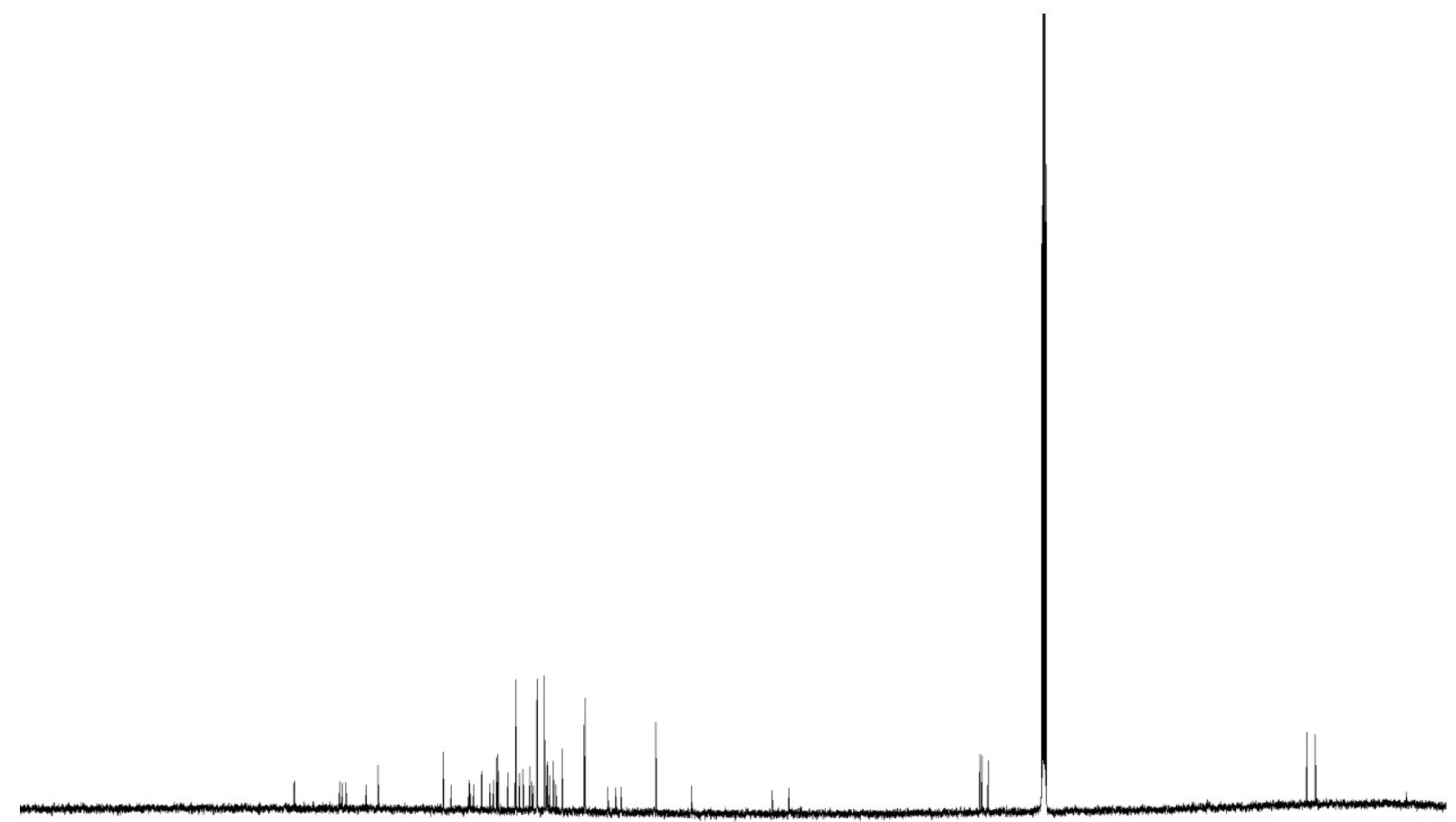

$\begin{array}{llllllllllllllllllll}190 & 180 & 170 & 160 & 150 & 140 & 130 & 120 & 110 & 100 & 90 & 80 & 70 & 60 & 50 & 40 & 30 & 20 & 10 & \mathrm{ppm}\end{array}$

Figure S80. ${ }^{13} \mathrm{C}\left\{{ }^{1} \mathrm{H}\right\}$ NMR of $\mathbf{1 8}(S)$ a in $\mathrm{CD}_{2} \mathrm{Cl}_{2}$ at a $176.1 \mathrm{MHz}$ spectrometer at $298 \mathrm{~K}$, Overview. 


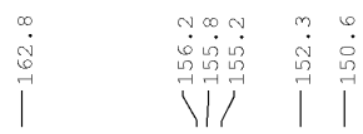

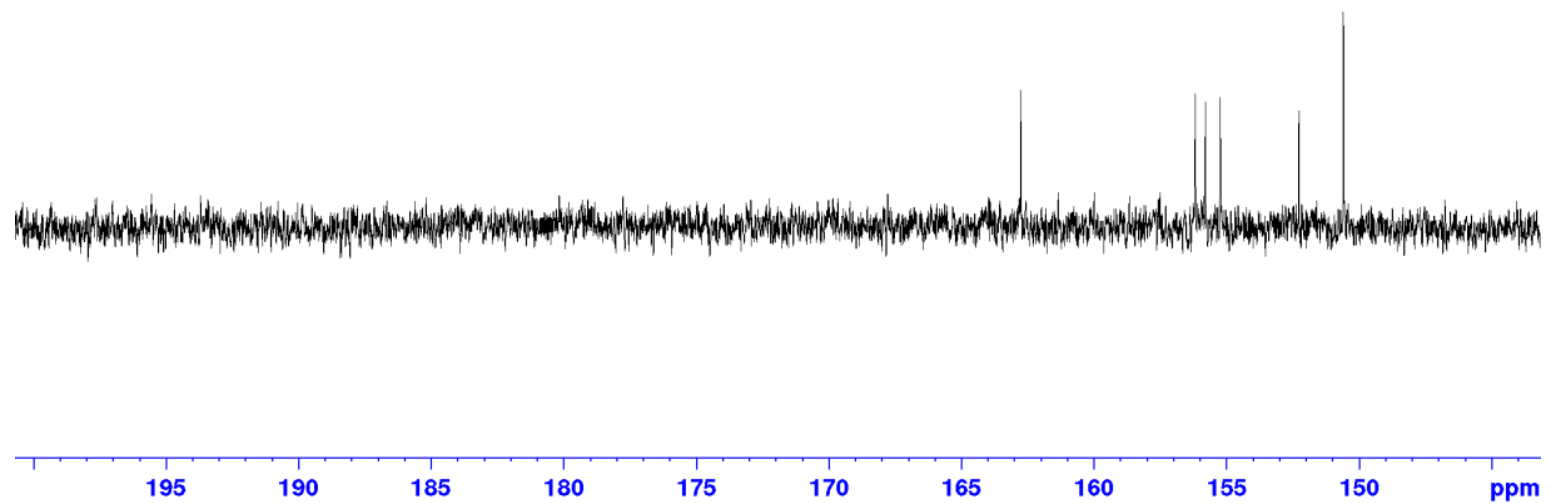

Figure S81. ${ }^{13} \mathrm{C}\left\{{ }^{1} \mathrm{H}\right\}$ NMR of $\mathbf{1 8}(\boldsymbol{S}) \mathbf{a}$ in $\mathrm{CD}_{2} \mathrm{Cl}_{2}$ at a $176.1 \mathrm{MHz}$ spectrometer at $298 \mathrm{~K}$, Part 1 (200 ppm - 144 ppm).
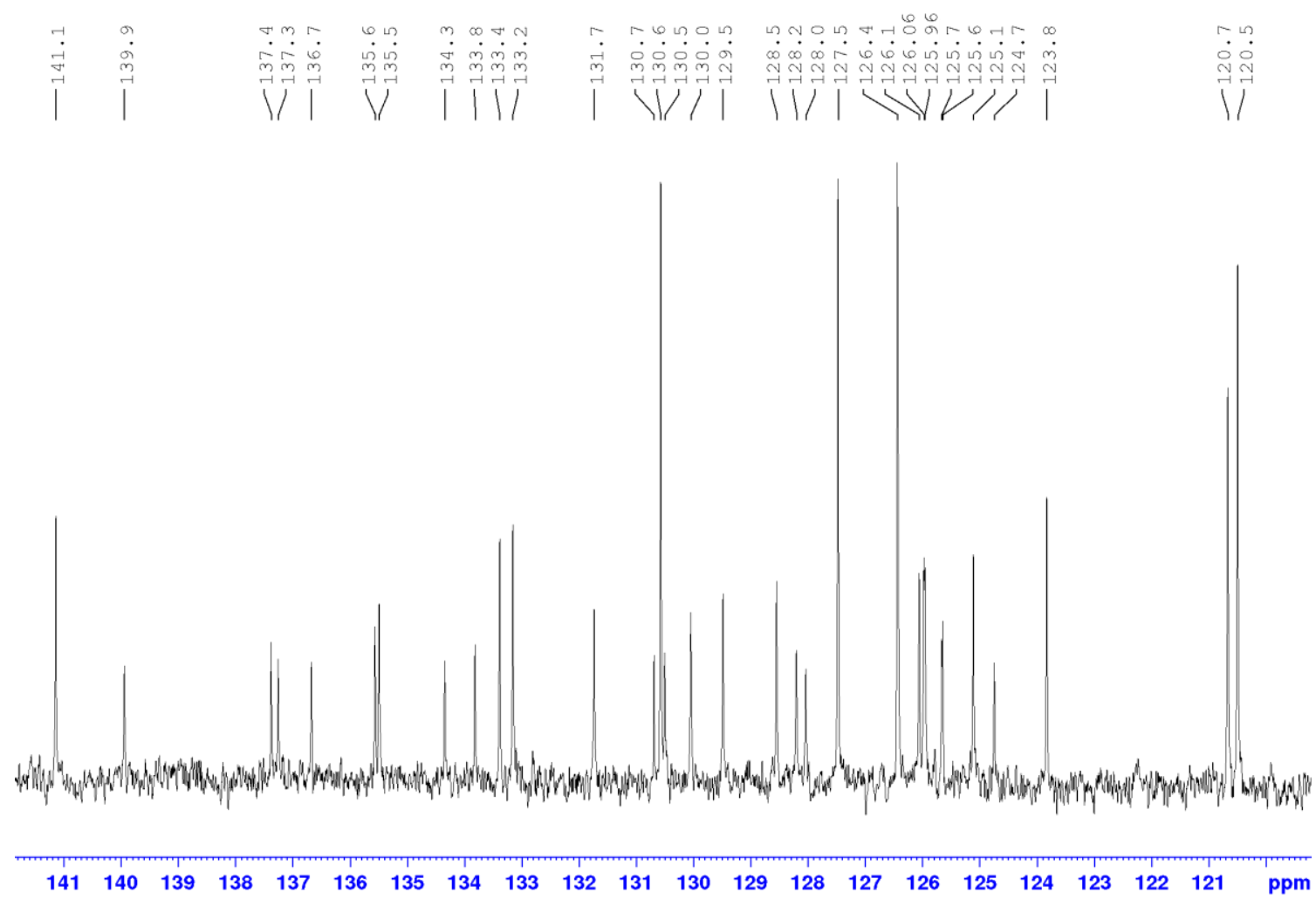

Figure S82. ${ }^{13} \mathrm{C}\left\{{ }^{1} \mathrm{H}\right\}$ NMR of $\mathbf{1 8}(S)$ a in $\mathrm{CD}_{2} \mathrm{Cl}_{2}$ at a $176.1 \mathrm{MHz}$ spectrometer at $298 \mathrm{~K}$, Part 2 (144 ppm - 119 ppm). 


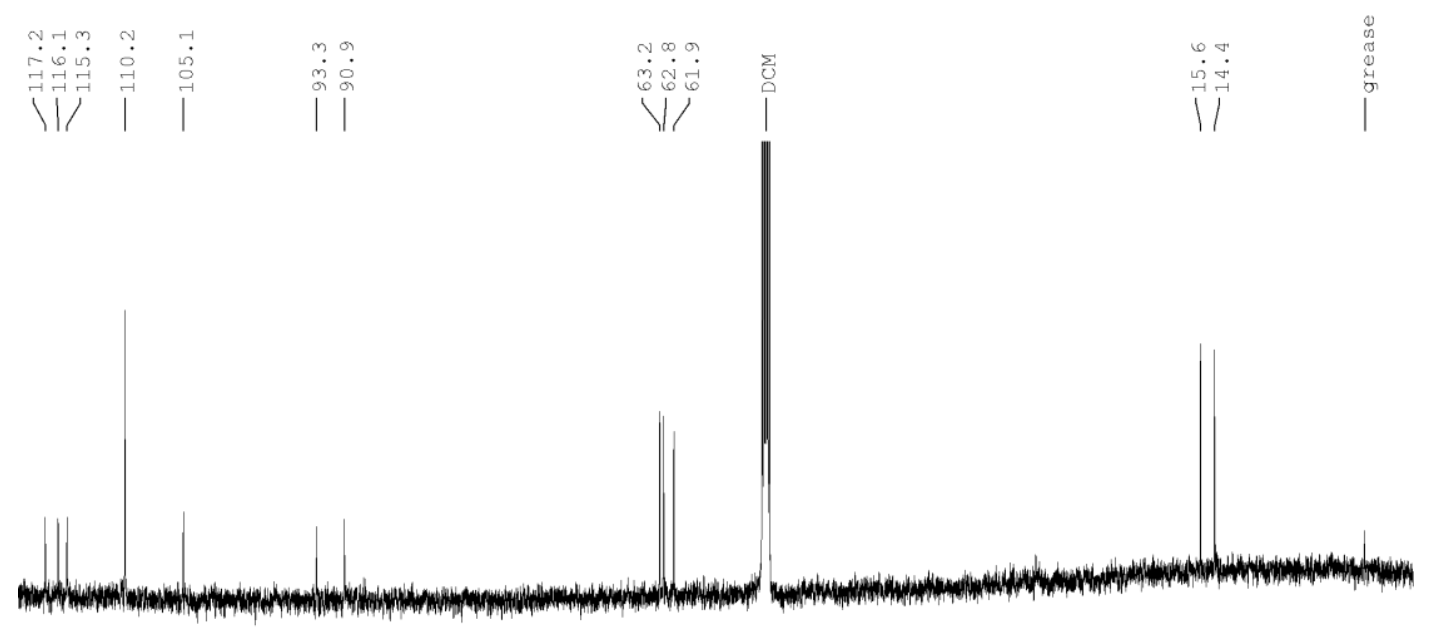

$\begin{array}{llllllllllllllllllllllll}115 & 110 & 105 & 100 & 95 & 90 & 85 & 80 & 75 & 70 & 65 & 60 & 55 & 50 & 45 & 40 & 35 & 30 & 25 & 20 & 15 & 10 & 5 & \mathrm{ppm}\end{array}$

Figure S83. ${ }^{13} \mathrm{C}\left\{{ }^{1} \mathrm{H}\right\}$ NMR of $\mathbf{1 8}(\boldsymbol{S}) \mathbf{a}$ in $\mathrm{CD}_{2} \mathrm{Cl}_{2}$ at a $176.1 \mathrm{MHz}$ spectrometer at $298 \mathrm{~K}$, Part 3 (119 ppm-0 ppm).

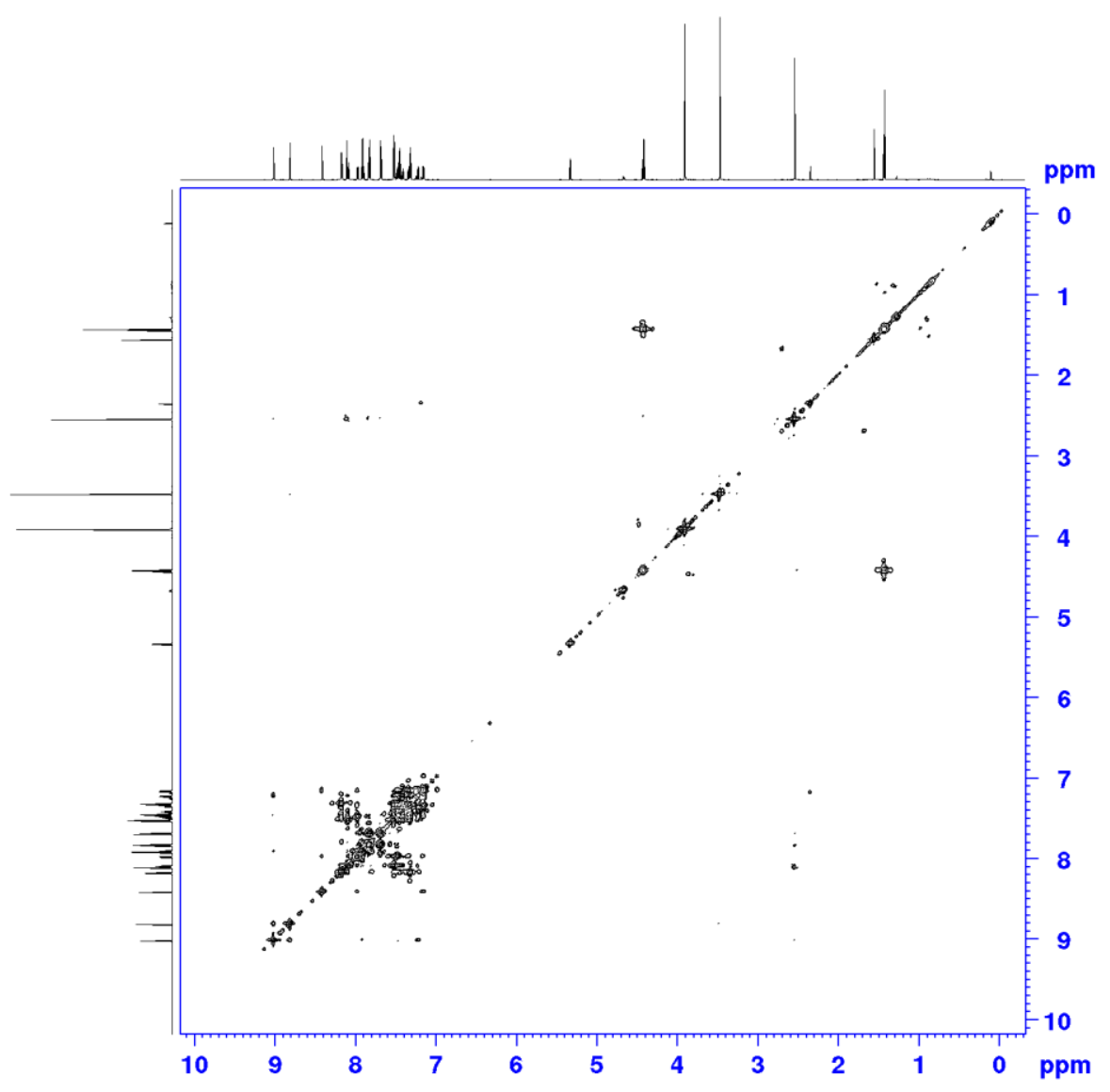

Figure S84. ${ }^{1} \mathrm{H},{ }^{1} \mathrm{H}$ gCOSY NMR spectrum of $\mathbf{1 8}(S)$ a in $\mathrm{CD}_{2} \mathrm{Cl}_{2}$ at a $700 \mathrm{MHz}$ Spectrometer at $298 \mathrm{~K}$. 


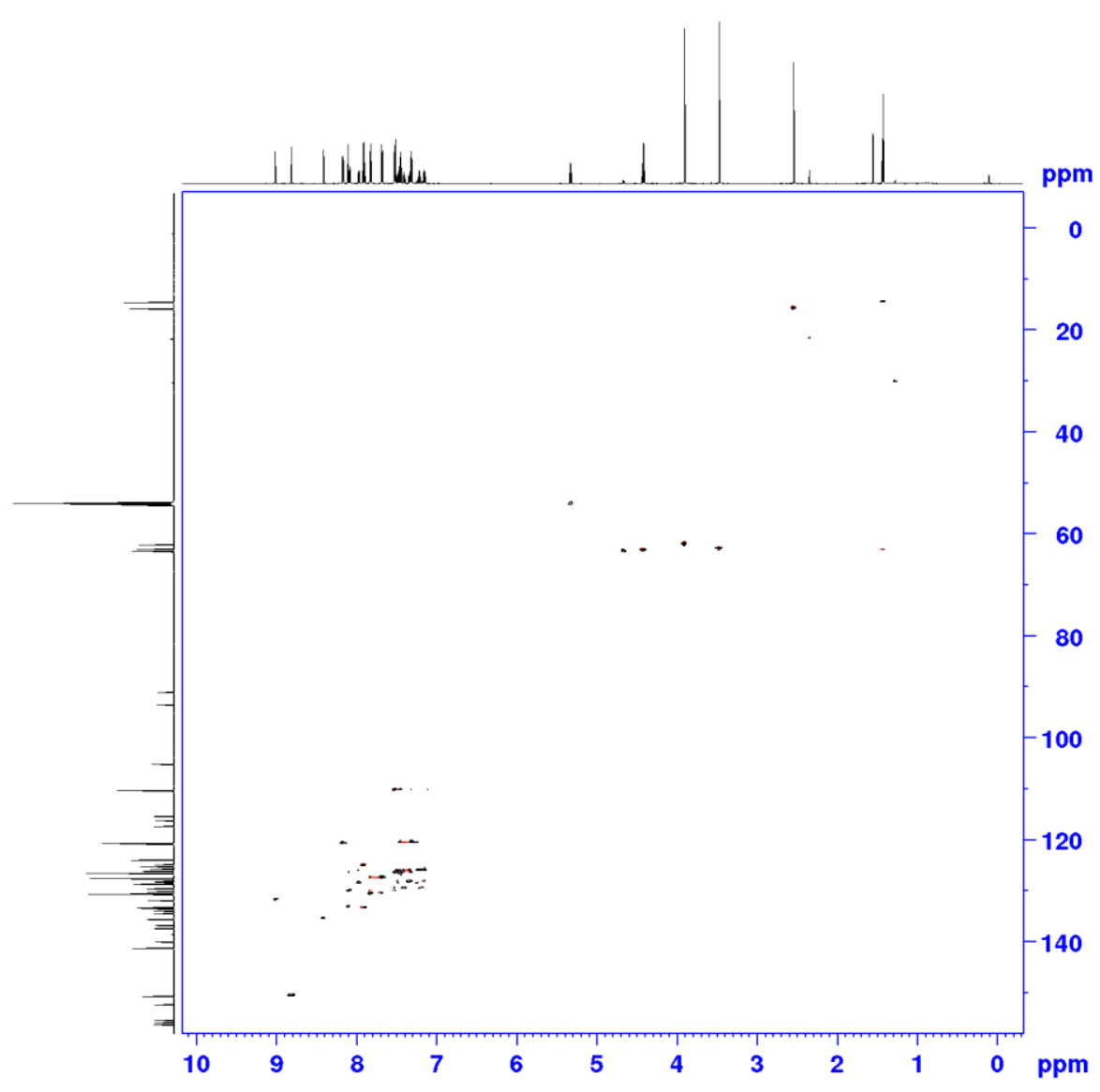

Figure S85. ${ }^{1} \mathrm{H},{ }^{13} \mathrm{C}$ gHSQC NMR spectrum of $\mathbf{1 8}(\boldsymbol{S}) \mathbf{a}$ in $\mathrm{CD}_{2} \mathrm{Cl}_{2}$ at a $700 \mathrm{MHz}$ Spectrometer at $298 \mathrm{~K}$.

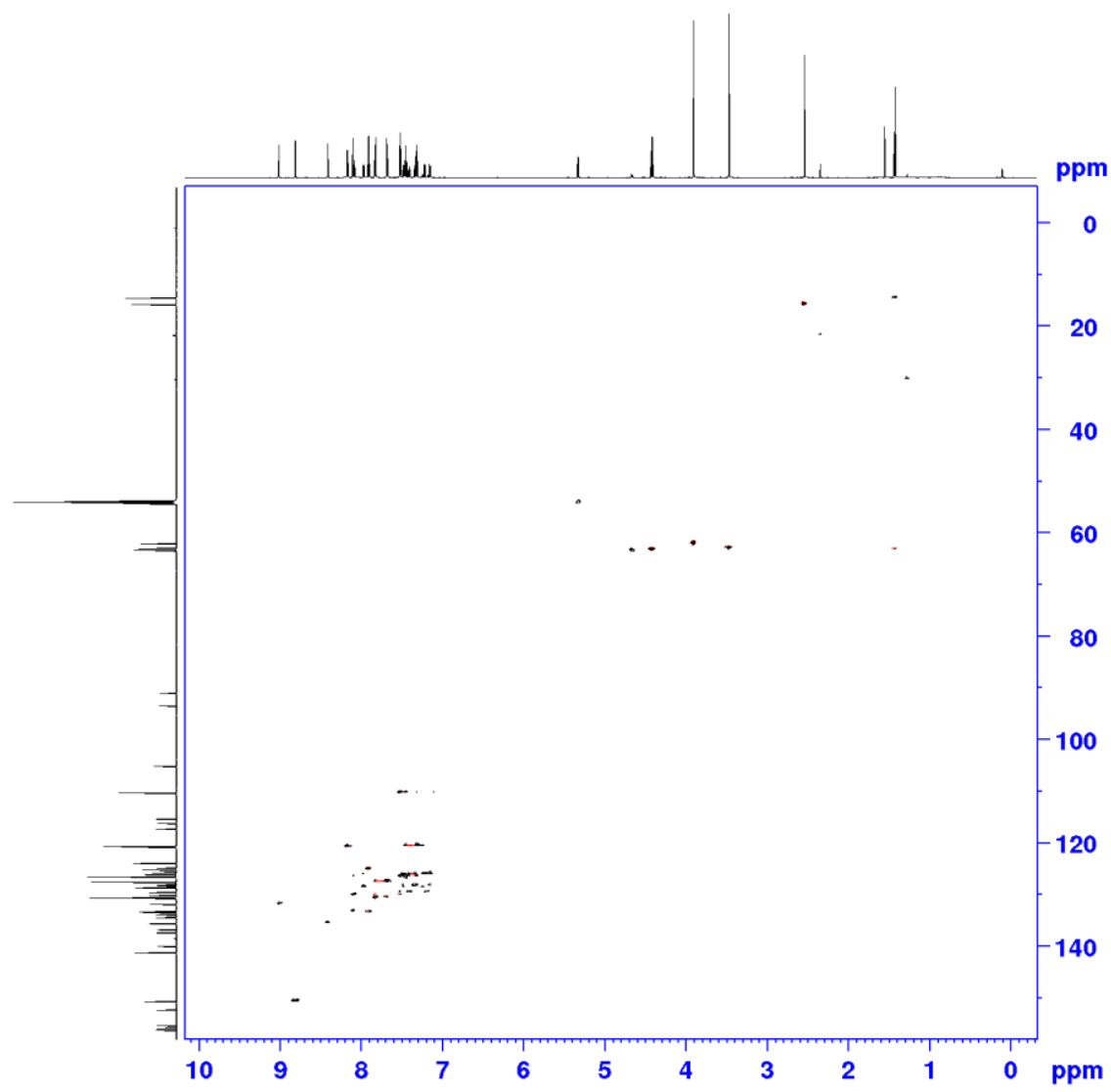

Figure S86. ${ }^{1} \mathrm{H},{ }^{13} \mathrm{C}$ gHMBC NMR spectrum of $\mathbf{1 8}(\mathrm{S}) \mathbf{a}$ in $\mathrm{CD}_{2} \mathrm{Cl}_{2}$ at a $700 \mathrm{MHz}$ Spectrometer at $298 \mathrm{~K}$. 

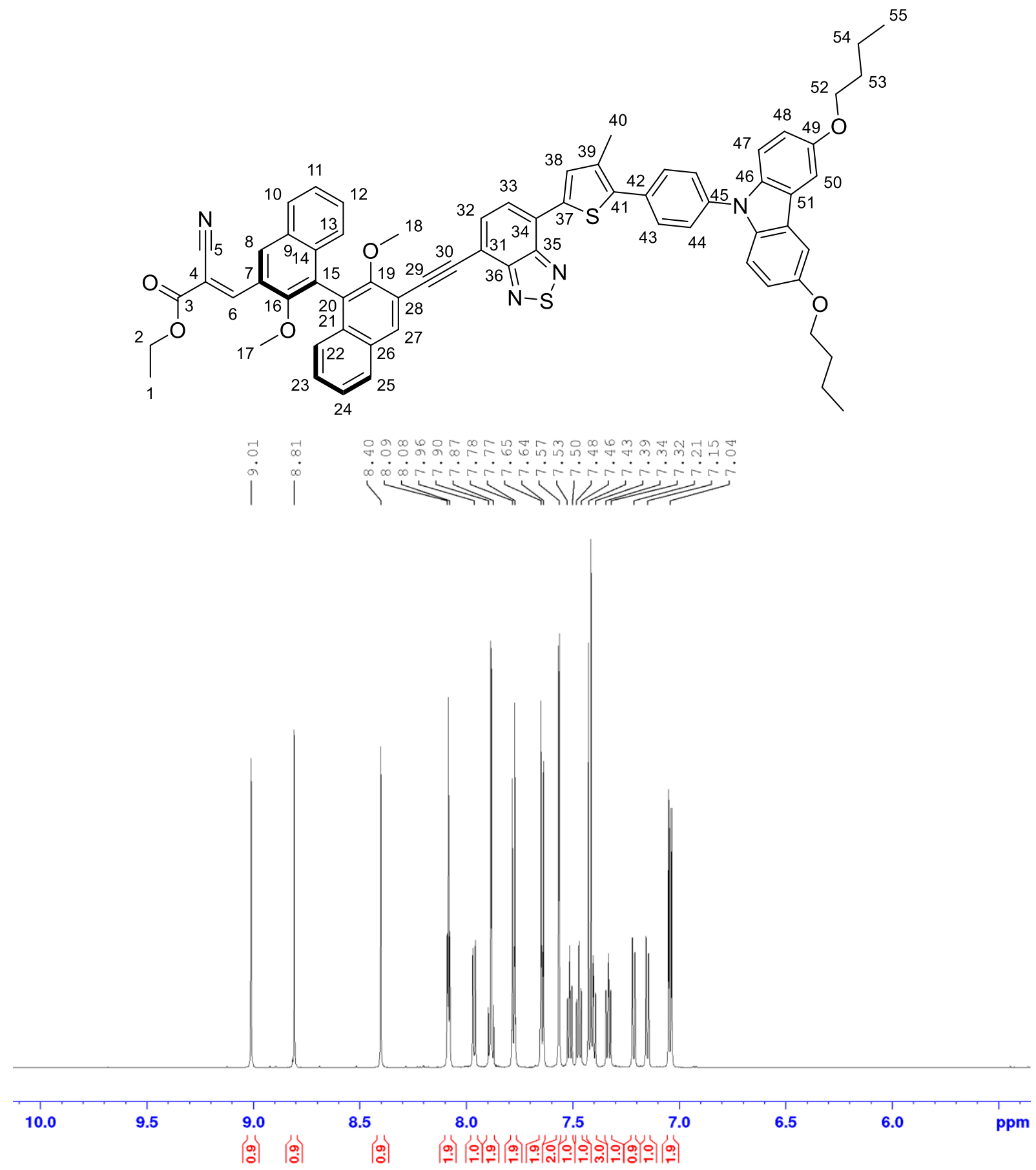

Figure S87. ${ }^{1} \mathrm{H}$ NMR of $\mathbf{1 8}(S)$ b in $\mathrm{CD}_{2} \mathrm{Cl}_{2}$ at a $700 \mathrm{MHz}$ spectrometer at $298 \mathrm{~K}$, Part 1 (10.4 ppm-5.4 ppm). 


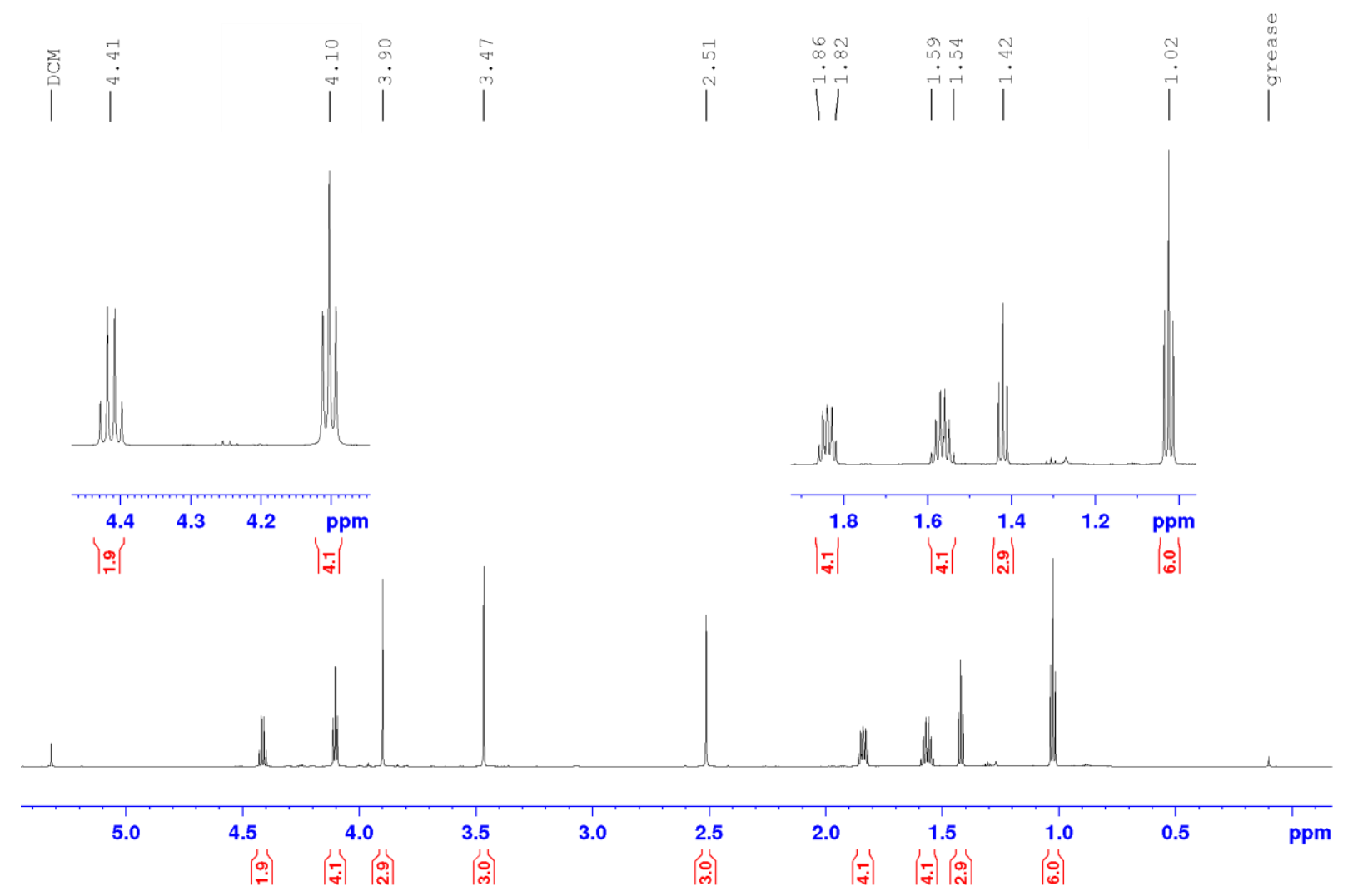

Figure S88. ${ }^{1} \mathrm{H}$ NMR of $\mathbf{1 8}(S)$ b in $\mathrm{CD}_{2} \mathrm{Cl}_{2}$ at a $700 \mathrm{MHz}$ spectrometer at $298 \mathrm{~K}$, Part 2 $(5.4 \mathrm{ppm}-0.0 \mathrm{ppm})$.

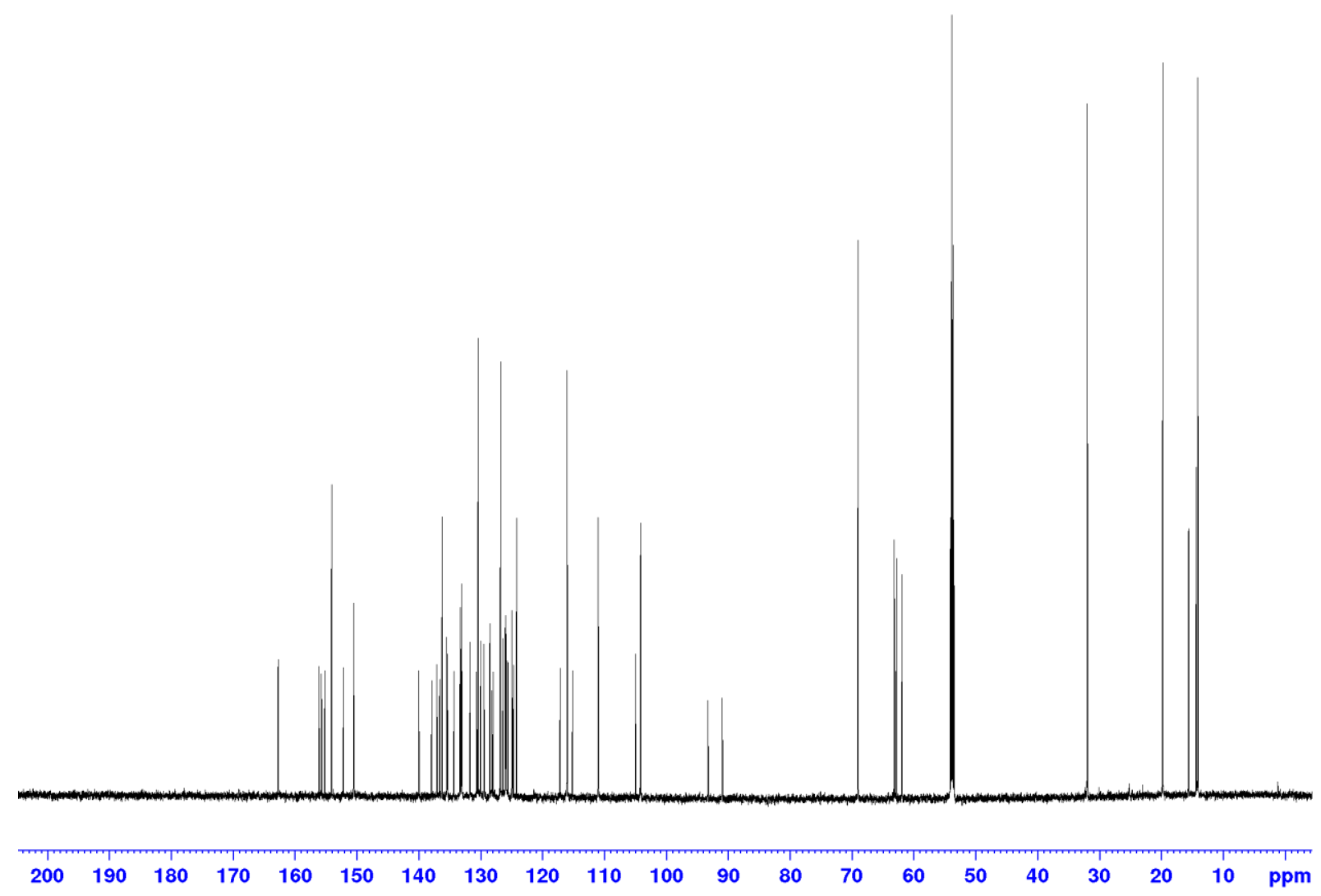

Figure S89. ${ }^{13} \mathrm{C}\left\{{ }^{1} \mathrm{H}\right\}$ NMR of $\mathbf{1 8}(\boldsymbol{S}) \mathbf{b}$ in $\mathrm{CD}_{2} \mathrm{Cl}_{2}$ at a $176.1 \mathrm{MHz}$ spectrometer at $298 \mathrm{~K}$, Overview. 


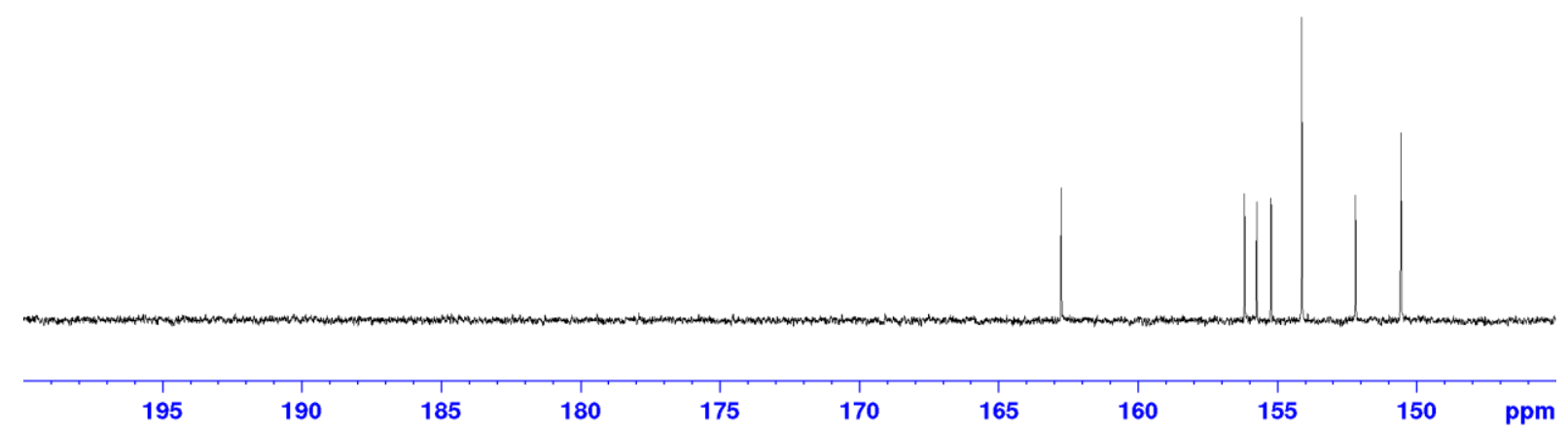

Figure S90. ${ }^{13} \mathrm{C}\left\{{ }^{1} \mathrm{H}\right\}$ NMR of $\mathbf{1 8}(S)$ b in $\mathrm{CD}_{2} \mathrm{Cl}_{2}$ at a $176.1 \mathrm{MHz}$ spectrometer at $298 \mathrm{~K}$, Part 1 (200 ppm - 145 ppm).
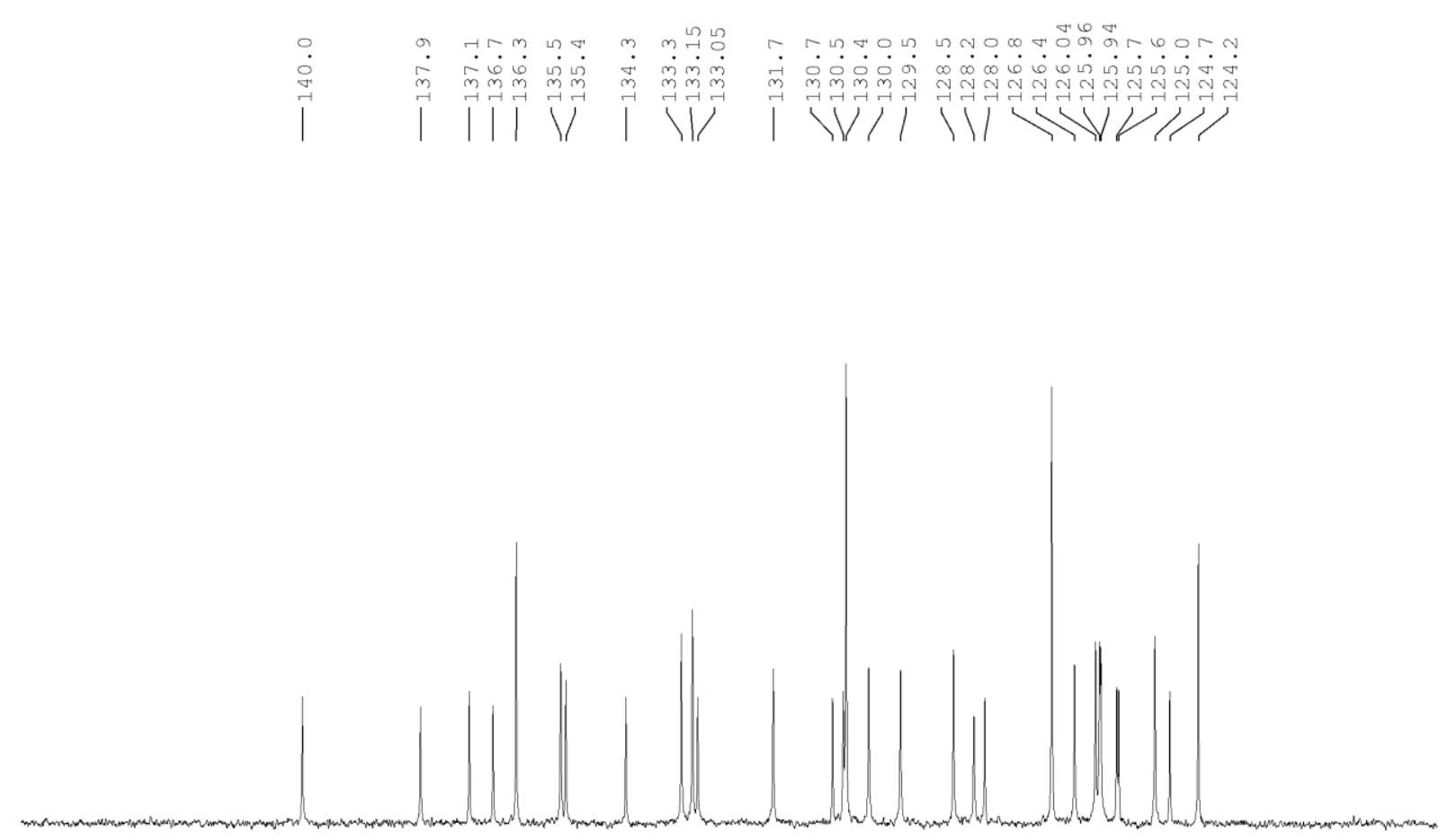

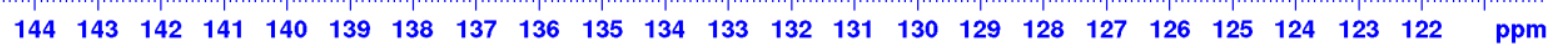

Figure S91. ${ }^{13} \mathrm{C}\left\{{ }^{1} \mathrm{H}\right\}$ NMR of $\mathbf{1 8}(S)$ b in $\mathrm{CD}_{2} \mathrm{Cl}_{2}$ at a $176.1 \mathrm{MHz}$ spectrometer at $298 \mathrm{~K}$, Part 2 $(145 \mathrm{ppm}-120 \mathrm{ppm})$. 


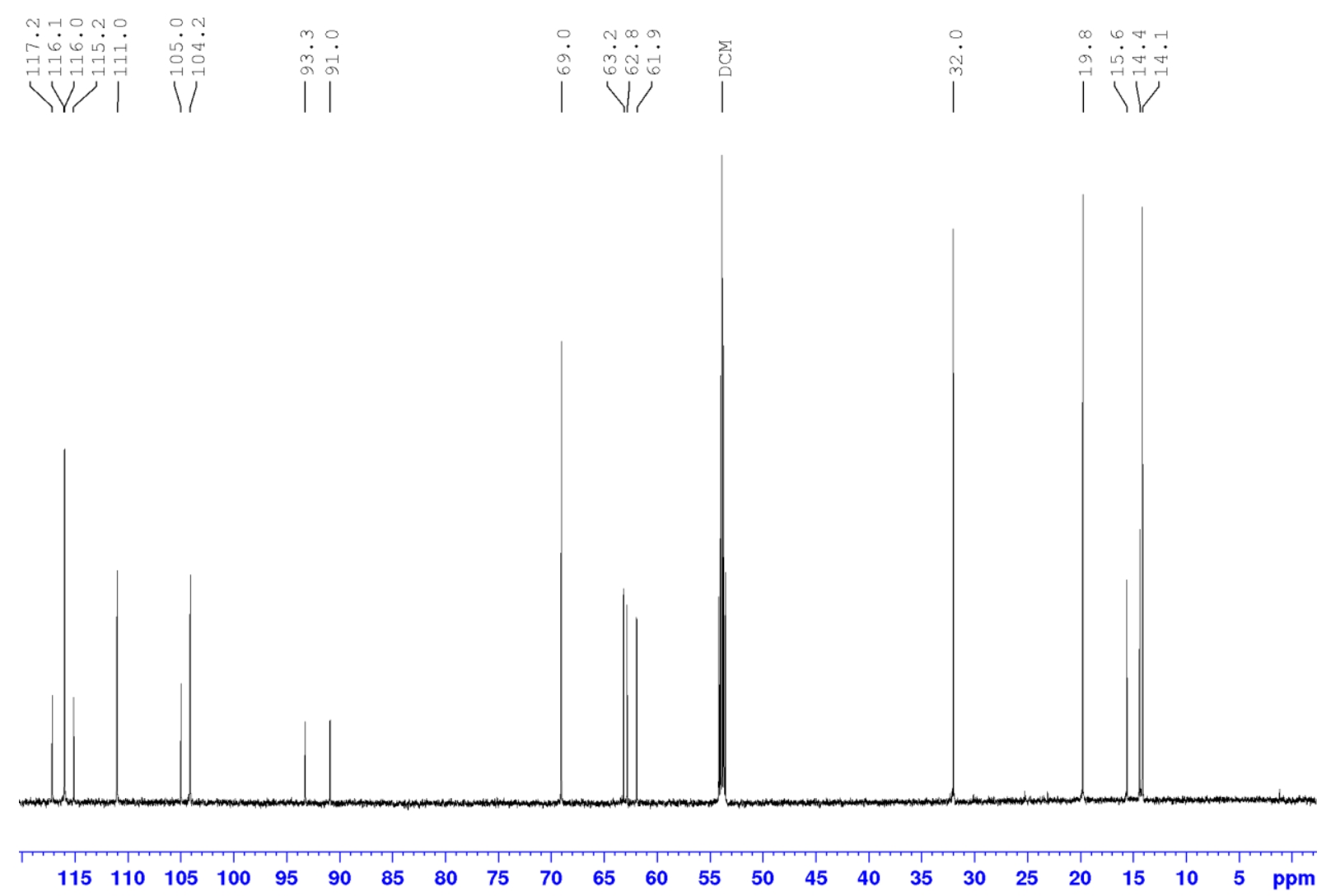

Figure S92. ${ }^{13} \mathrm{C}\left\{{ }^{1} \mathrm{H}\right\}$ NMR of $\mathbf{1 8}(S)$ b in $\mathrm{CD}_{2} \mathrm{Cl}_{2}$ at a $176.1 \mathrm{MHz}$ spectrometer at $298 \mathrm{~K}$, Part 3 (120 ppm-0 ppm).

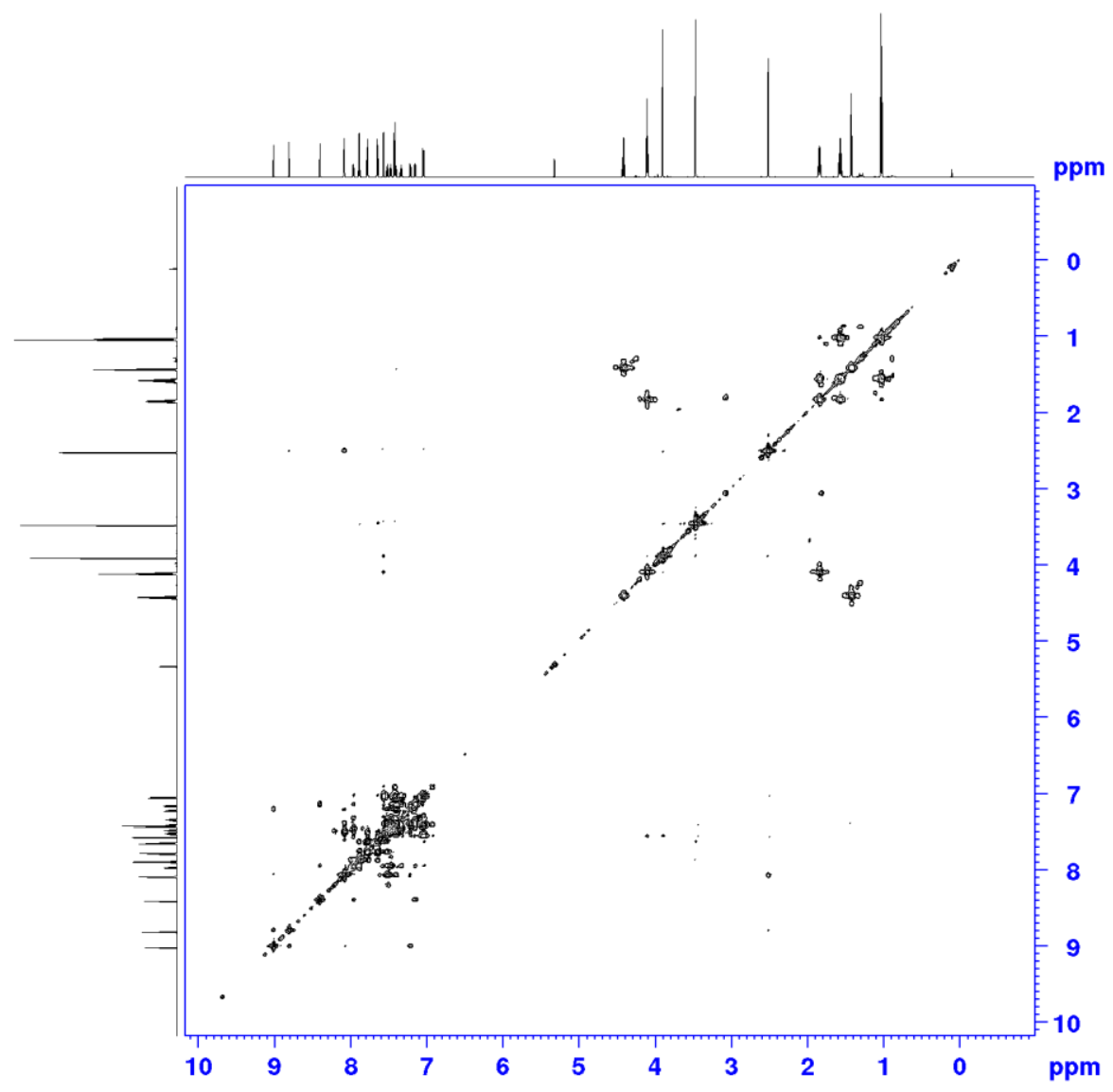

Figure S93. ${ }^{1} \mathrm{H},{ }^{1} \mathrm{H}$ gCOSY NMR spectrum of $\mathbf{1 8}(\boldsymbol{S}) \mathbf{b}$ in $\mathrm{CD}_{2} \mathrm{Cl}_{2}$ at a $700 \mathrm{MHz}$ spectrometer at $298 \mathrm{~K}$. 


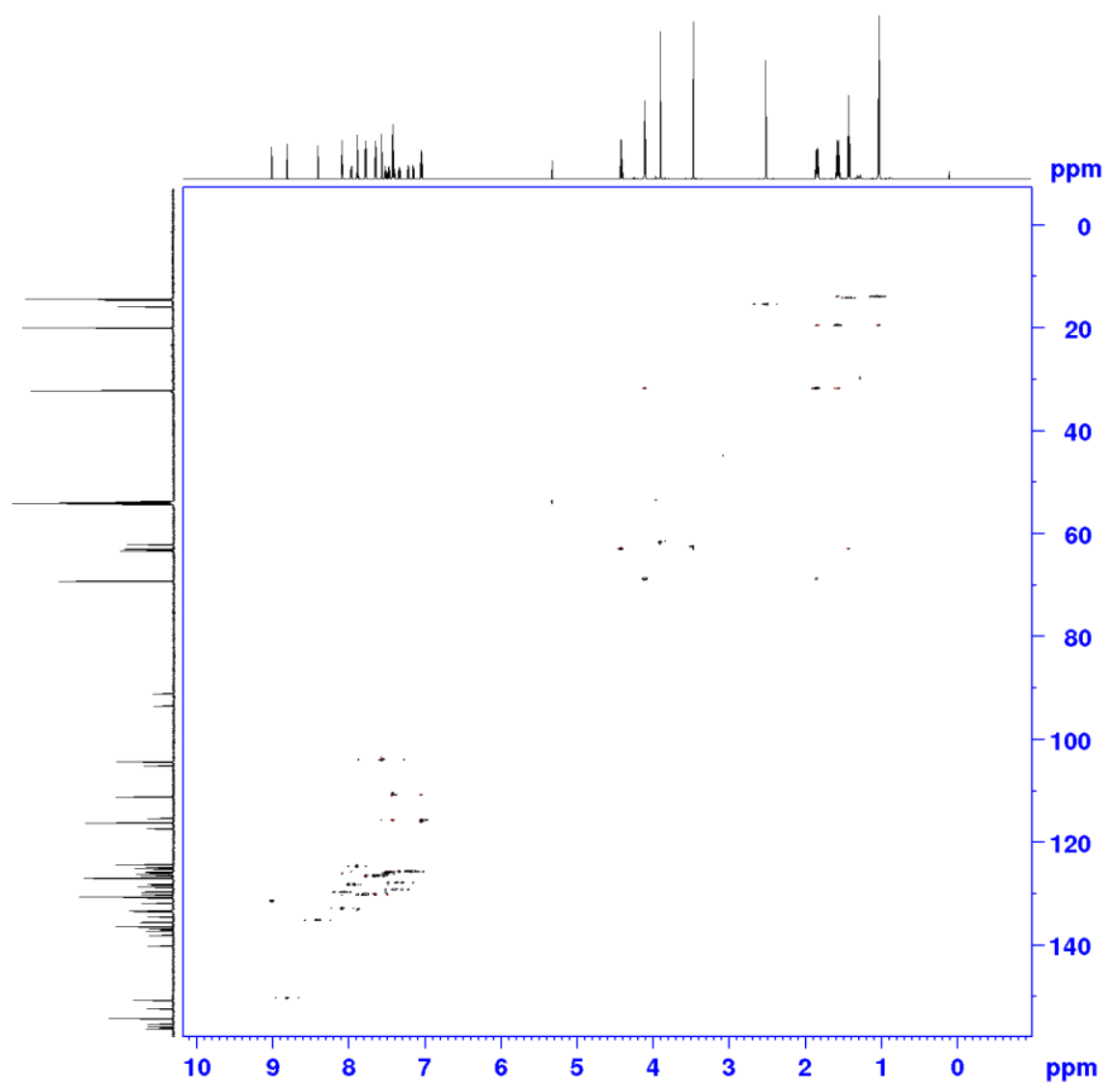

Figure S94. ${ }^{1} \mathrm{H},{ }^{13} \mathrm{C}$ gHSQC NMR spectrum of $\mathbf{1 8}(\boldsymbol{S}) \mathbf{b}$ in $\mathrm{CD}_{2} \mathrm{Cl}_{2}$ at a $700 \mathrm{MHz}$ spectrometer at $298 \mathrm{~K}$.

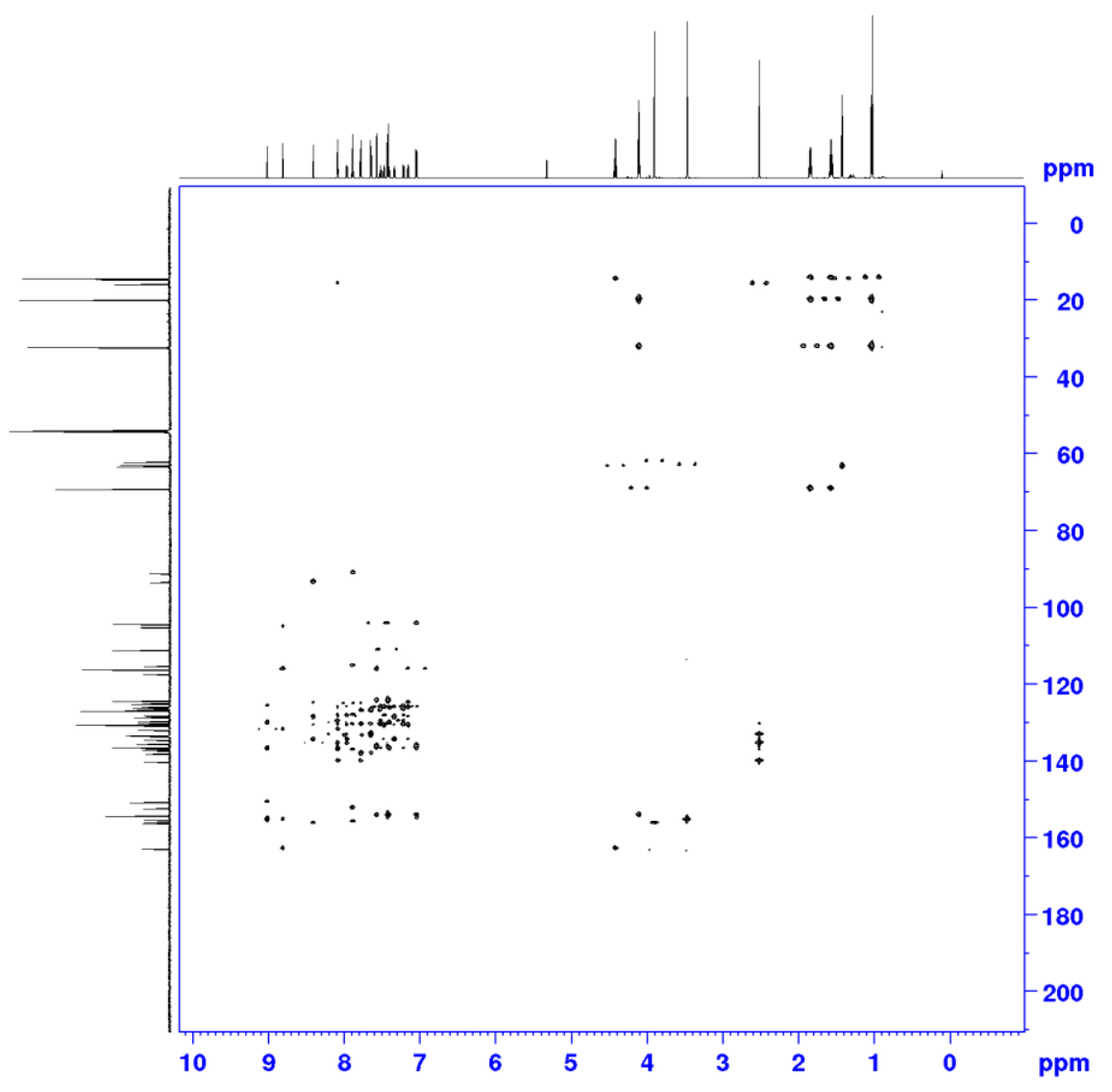

Figure S95. ${ }^{1} \mathrm{H},{ }^{13} \mathrm{C}$ gHMBC NMR spectrum of $\mathbf{1 8}(\boldsymbol{S}) \mathbf{b}$ in $\mathrm{CD}_{2} \mathrm{Cl}_{2}$ at a $700 \mathrm{MHz}$ spectrometer at $298 \mathrm{~K}$. 

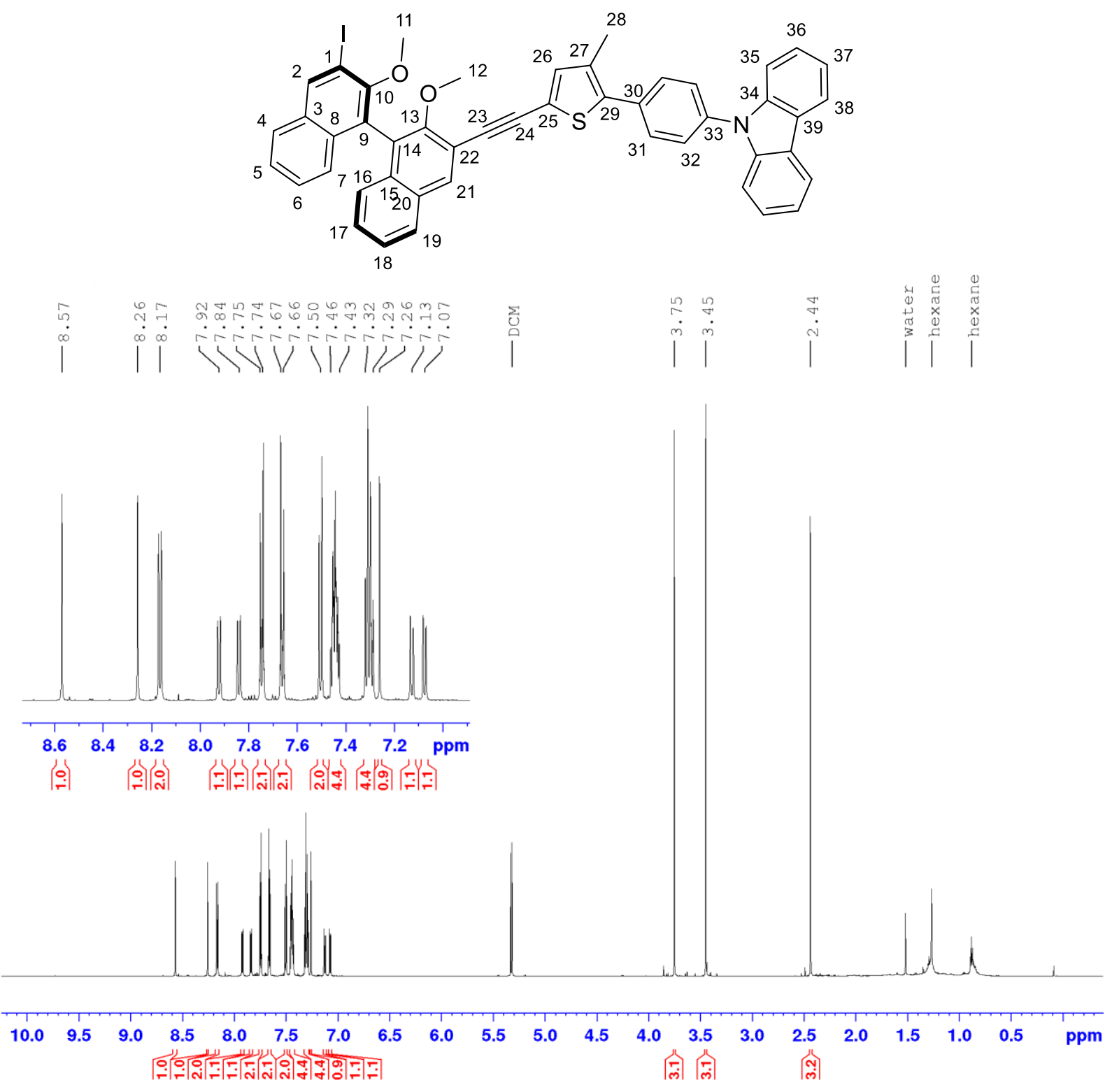

Figure S96. ${ }^{1} \mathrm{H}$ NMR of $\mathbf{2 0}(S)$ in $\mathrm{CD}_{2} \mathrm{Cl}_{2}$ at a $700 \mathrm{MHz}$ spectrometer at $298 \mathrm{~K}$. 


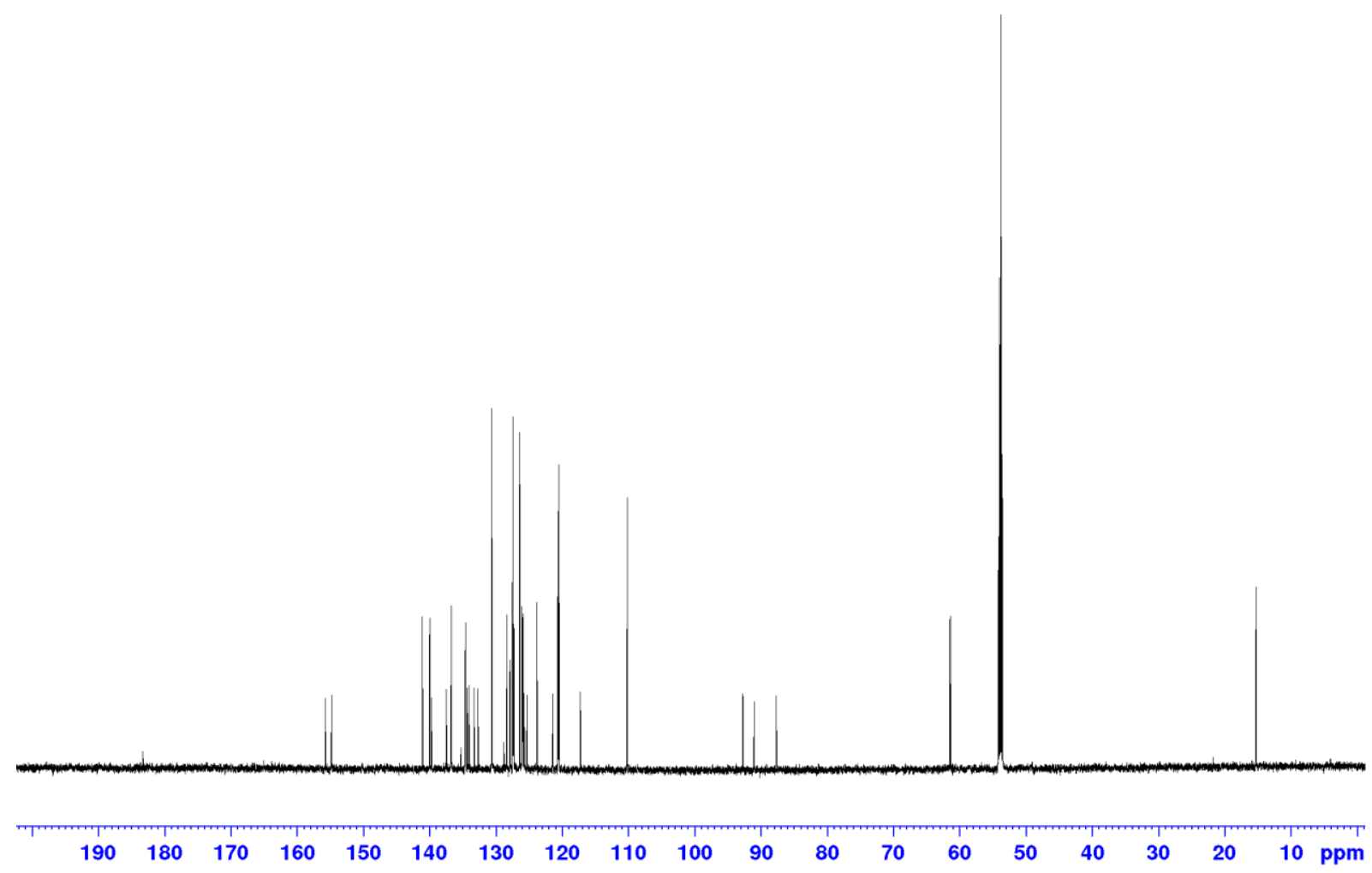

Figure S97. ${ }^{13} \mathrm{C}\left\{{ }^{1} \mathrm{H}\right\}$ NMR of $\mathbf{2 0}(S)$ in $\mathrm{CD}_{2} \mathrm{Cl}_{2}$ at a $176.1 \mathrm{MHz}$ spectrometer at $298 \mathrm{~K}$, Overview.

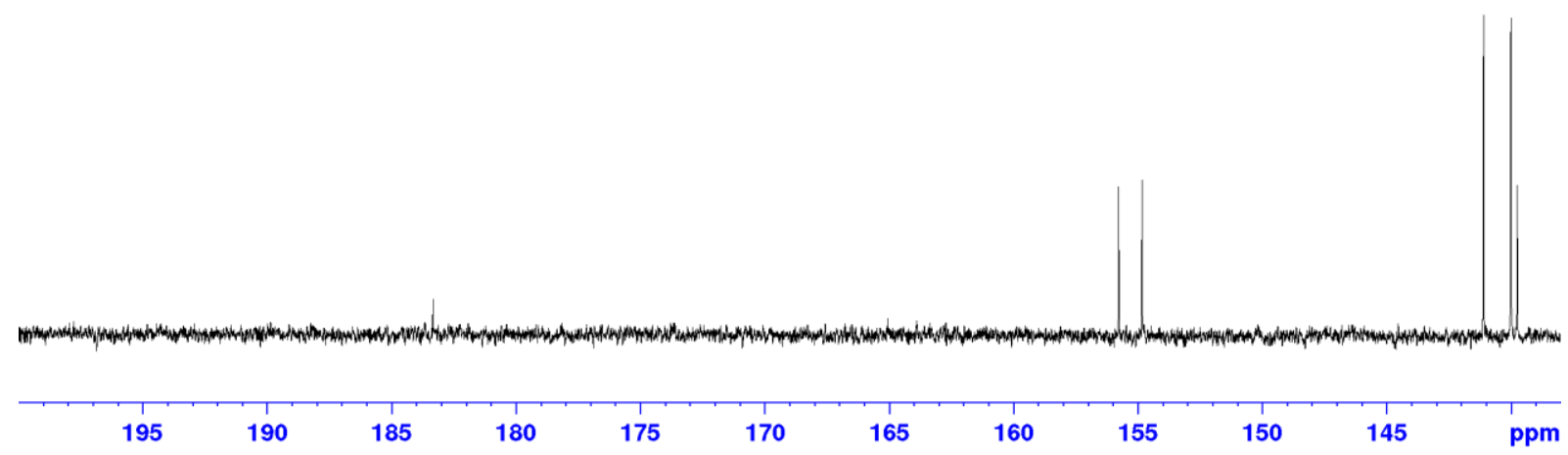

Figure S98. ${ }^{13} \mathrm{C}\left\{{ }^{1} \mathrm{H}\right\}$ NMR of $\mathbf{2 0}(S)$ in $\mathrm{CD}_{2} \mathrm{Cl}_{2}$ at a $176.1 \mathrm{MHz}$ spectrometer at $298 \mathrm{~K}$, Part 1 (200 ppm - 138 ppm). 


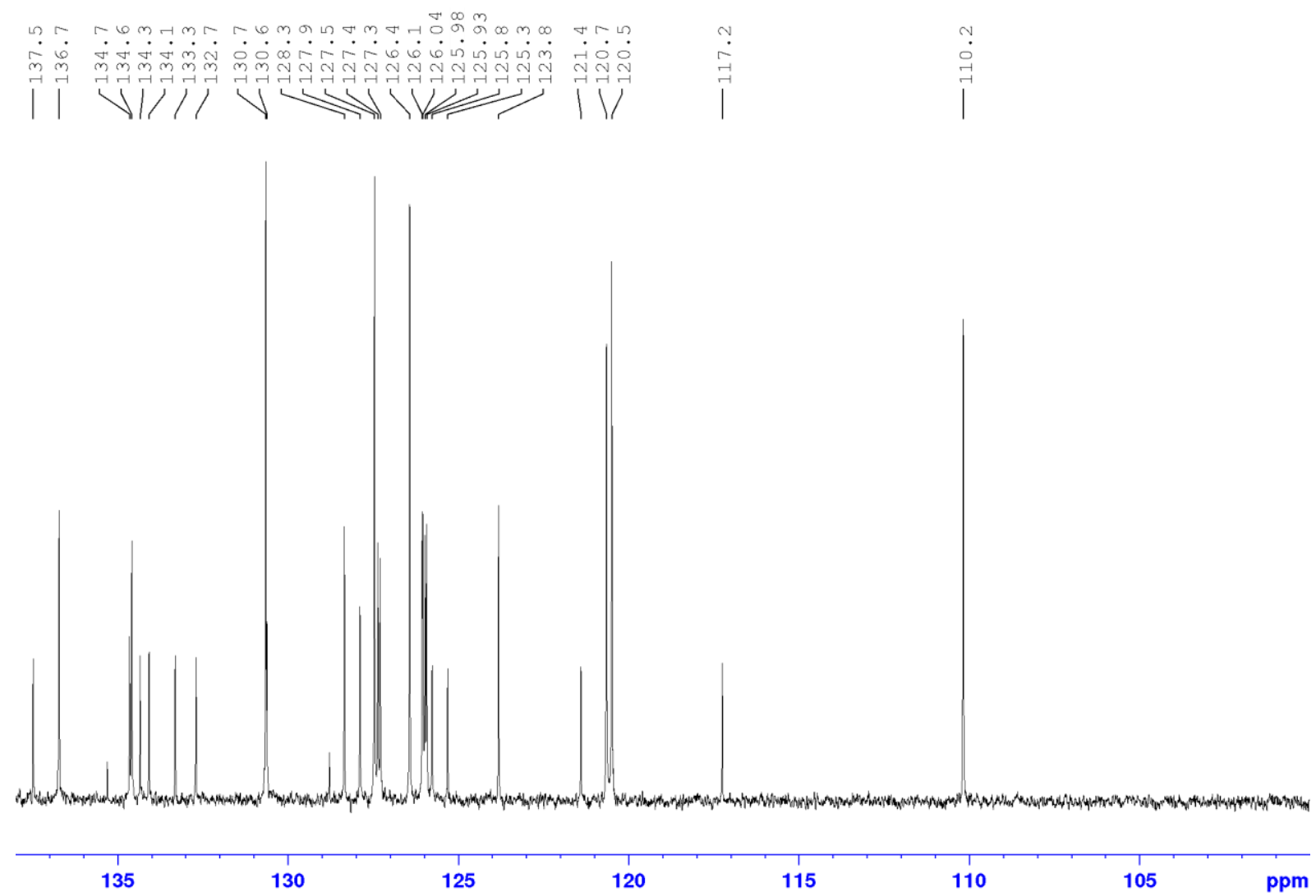

Figure S99. ${ }^{13} \mathrm{C}\left\{{ }^{1} \mathrm{H}\right\}$ NMR of $\mathbf{2 0}(\boldsymbol{S})$ in $\mathrm{CD}_{2} \mathrm{Cl}_{2}$ at a $176.1 \mathrm{MHz}$ spectrometer at $298 \mathrm{~K}$, Part 2 (138 ppm-100 ppm).

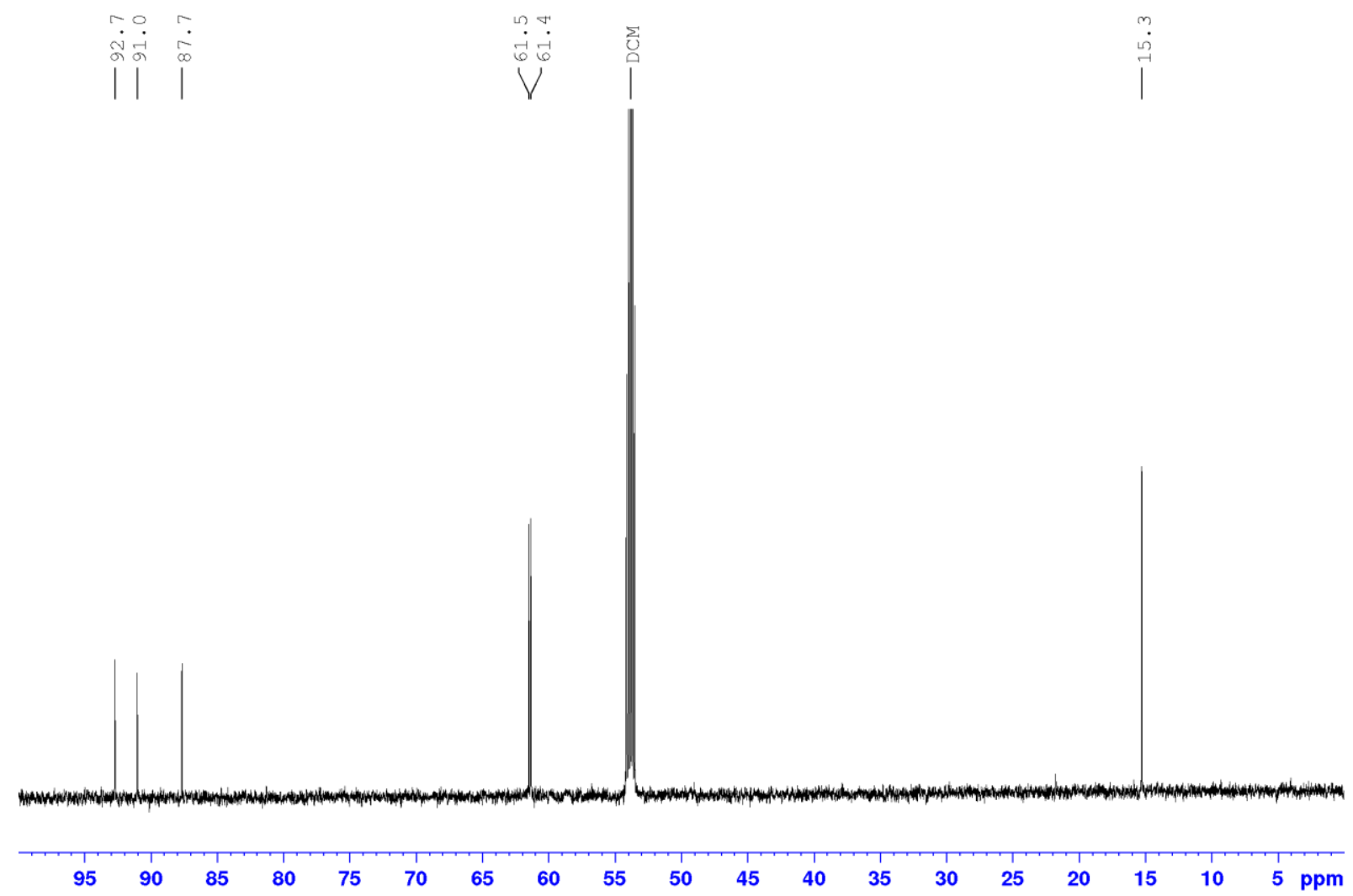

Figure S100. ${ }^{13} \mathrm{C}\left\{{ }^{1} \mathrm{H}\right\}$ NMR of $\mathbf{2 0}(S)$ in $\mathrm{CD}_{2} \mathrm{Cl}_{2}$ at a $176.1 \mathrm{MHz}$ spectrometer at $298 \mathrm{~K}$, Part 3 (100 ppm-0 ppm). 


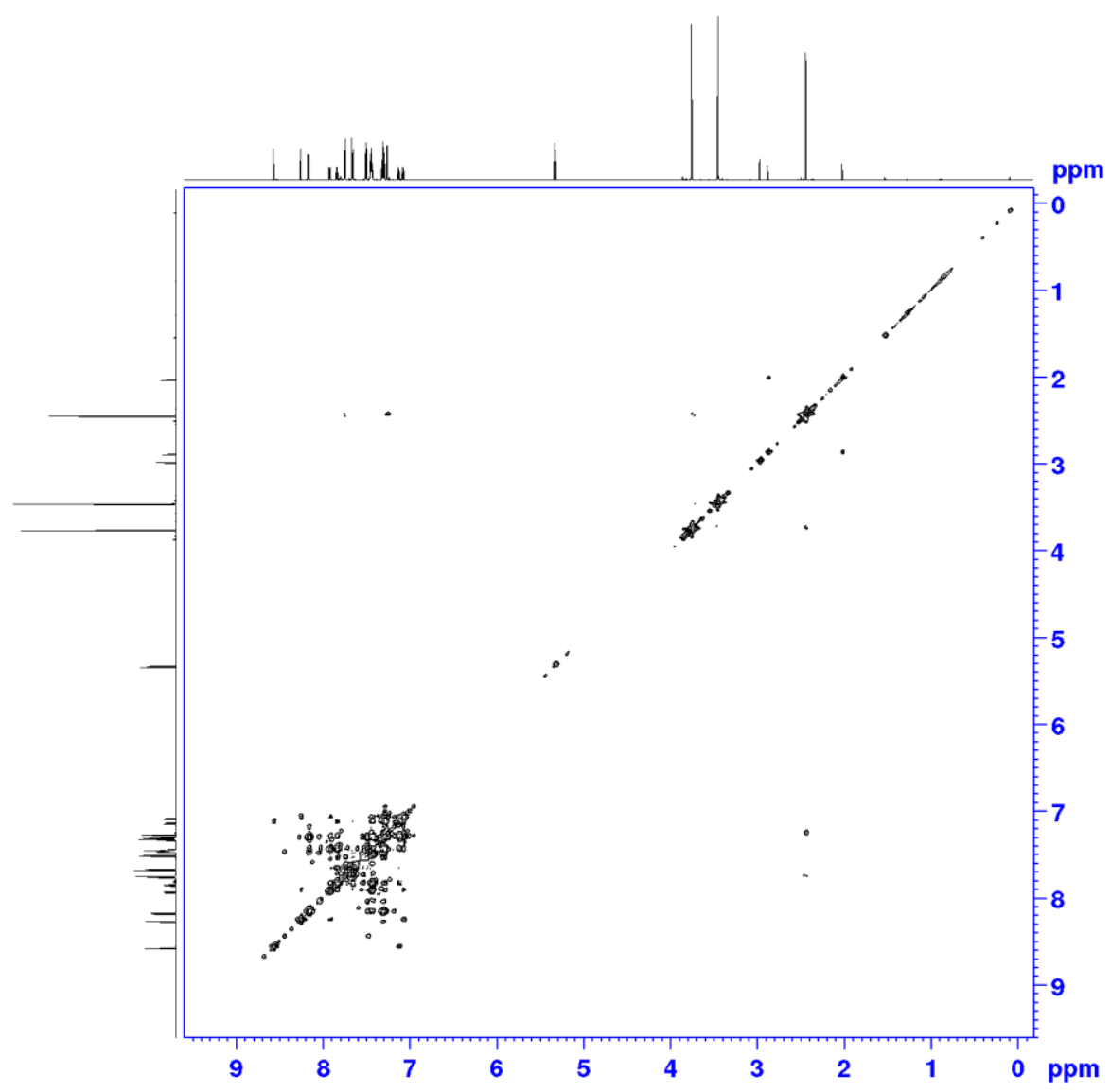

Figure S101. ${ }^{1} \mathrm{H},{ }^{1} \mathrm{H}$ gCOSY NMR spectrum of $\mathbf{2 0}(S)$ in $\mathrm{CD}_{2} \mathrm{Cl}_{2}$ at a $700 \mathrm{MHz}$ spectrometer at $298 \mathrm{~K}$.

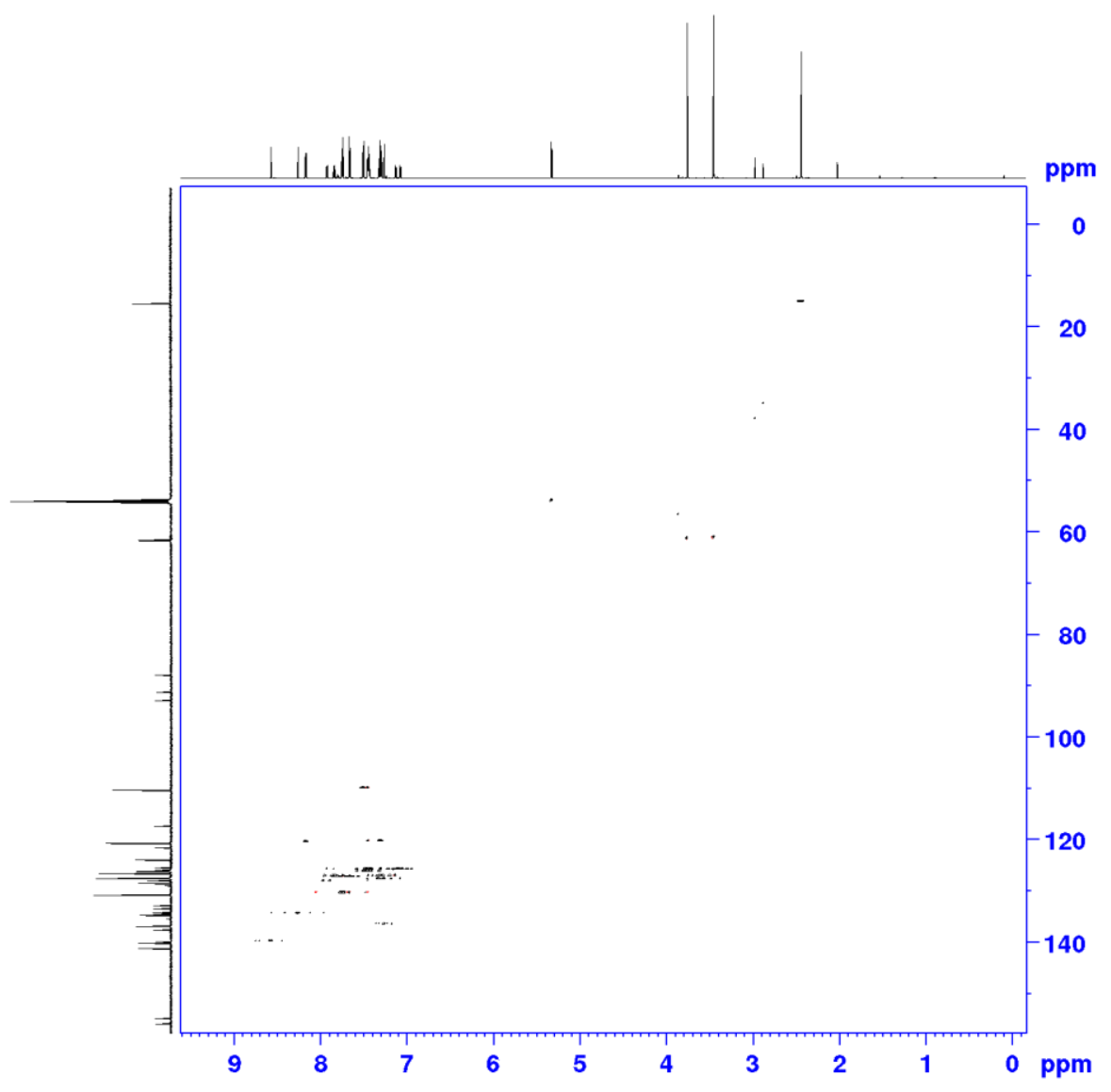

Figure S102. ${ }^{1} \mathrm{H},{ }^{13} \mathrm{C}$ gHSQC NMR spectrum of $\mathbf{2 0}(\boldsymbol{S})$ in $\mathrm{CD}_{2} \mathrm{Cl}_{2}$ at a $700 \mathrm{MHz}$ spectrometer at $298 \mathrm{~K}$. 


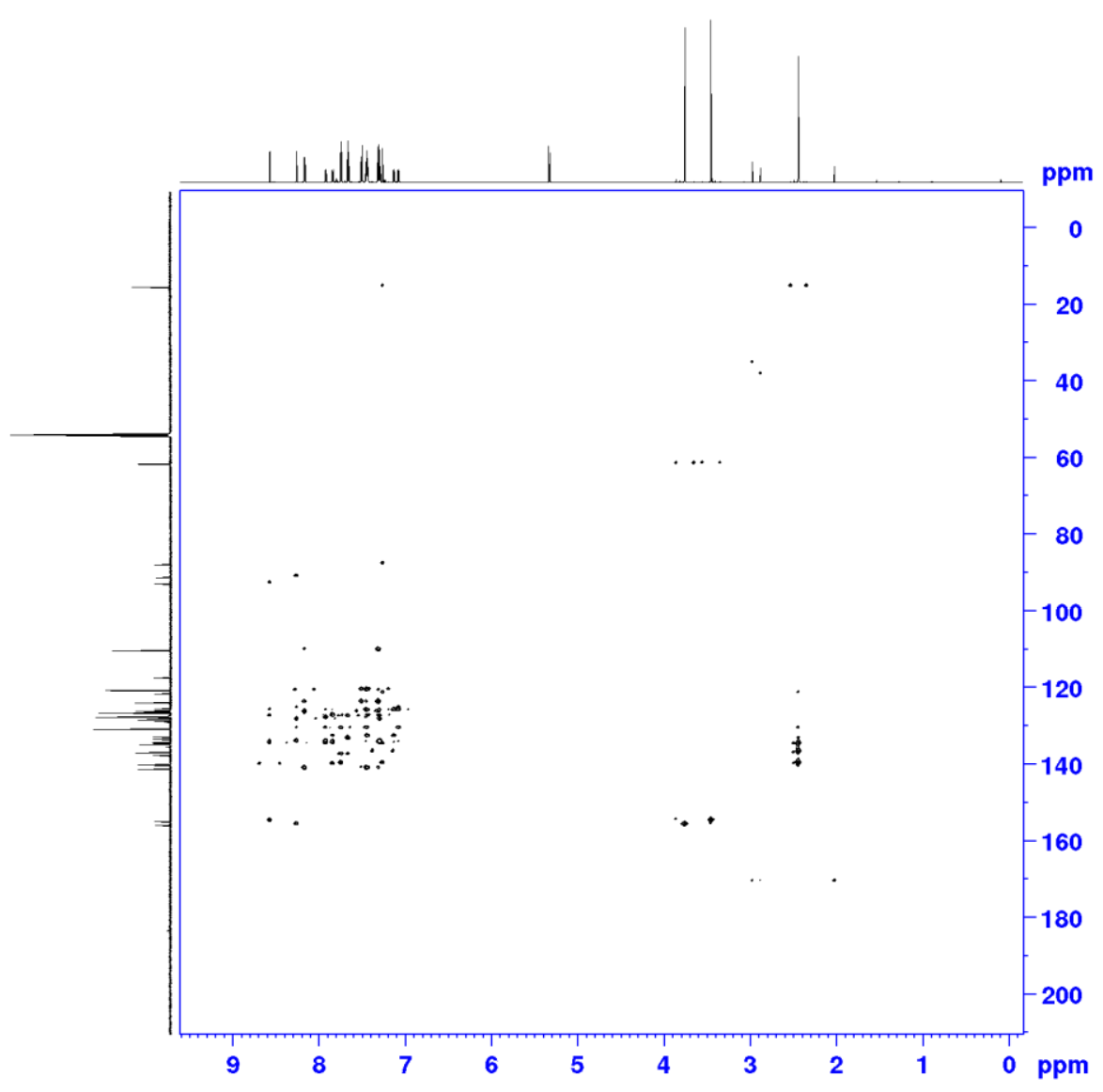

Figure S103. ${ }^{1} \mathrm{H},{ }^{13} \mathrm{C}$ gHMBC NMR spectrum of $\mathbf{2 0}(\boldsymbol{S})$ in $\mathrm{CD}_{2} \mathrm{Cl}_{2}$ at a $700 \mathrm{MHz}$ spectrometer at $298 \mathrm{~K}$. 

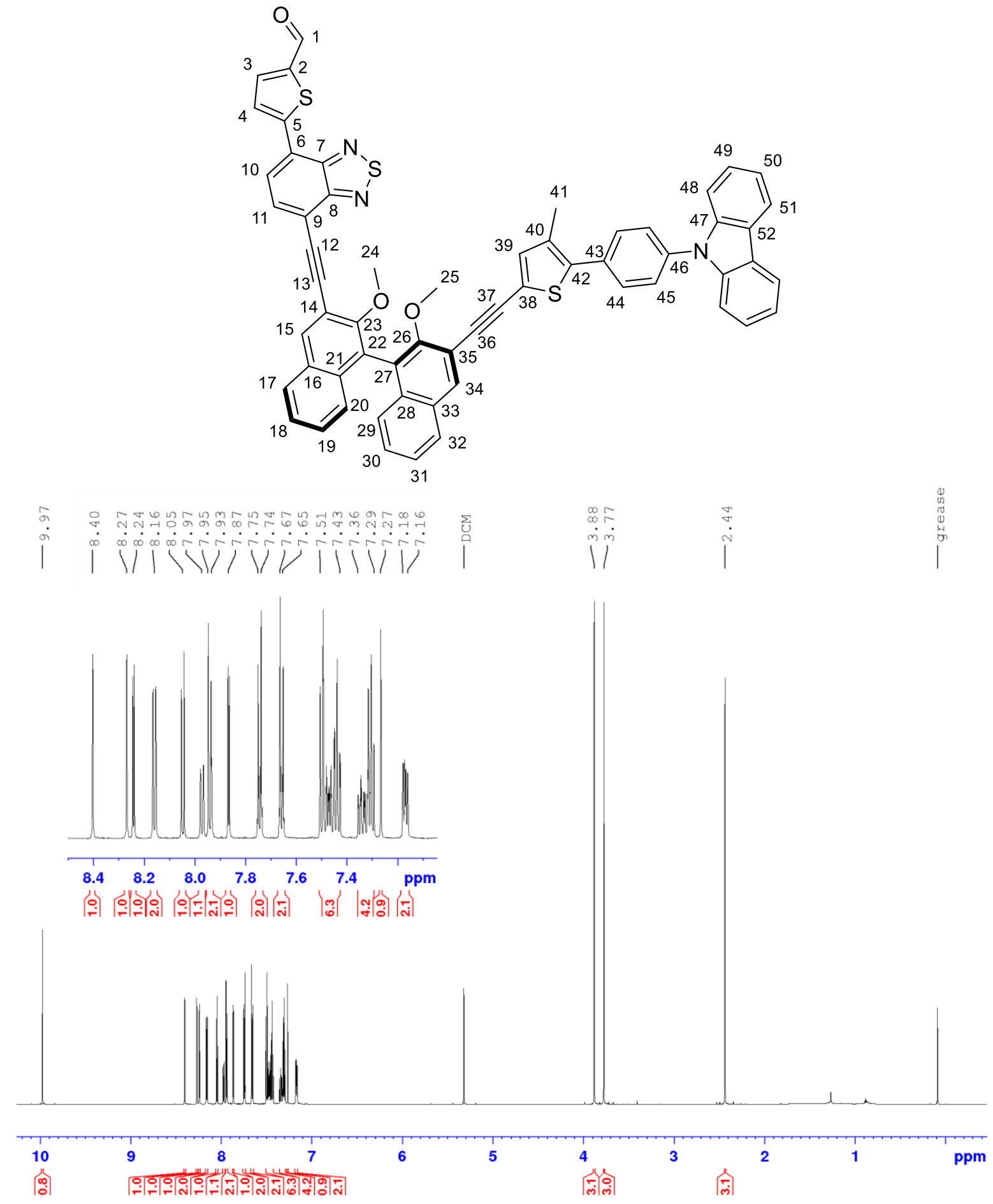

Figure S104. ${ }^{1} \mathrm{H}$ NMR of $\mathbf{2 1}(S)$ in $\mathrm{CD}_{2} \mathrm{Cl}_{2}$ at a $700 \mathrm{MHz}$ spectrometer at $298 \mathrm{~K}$. 


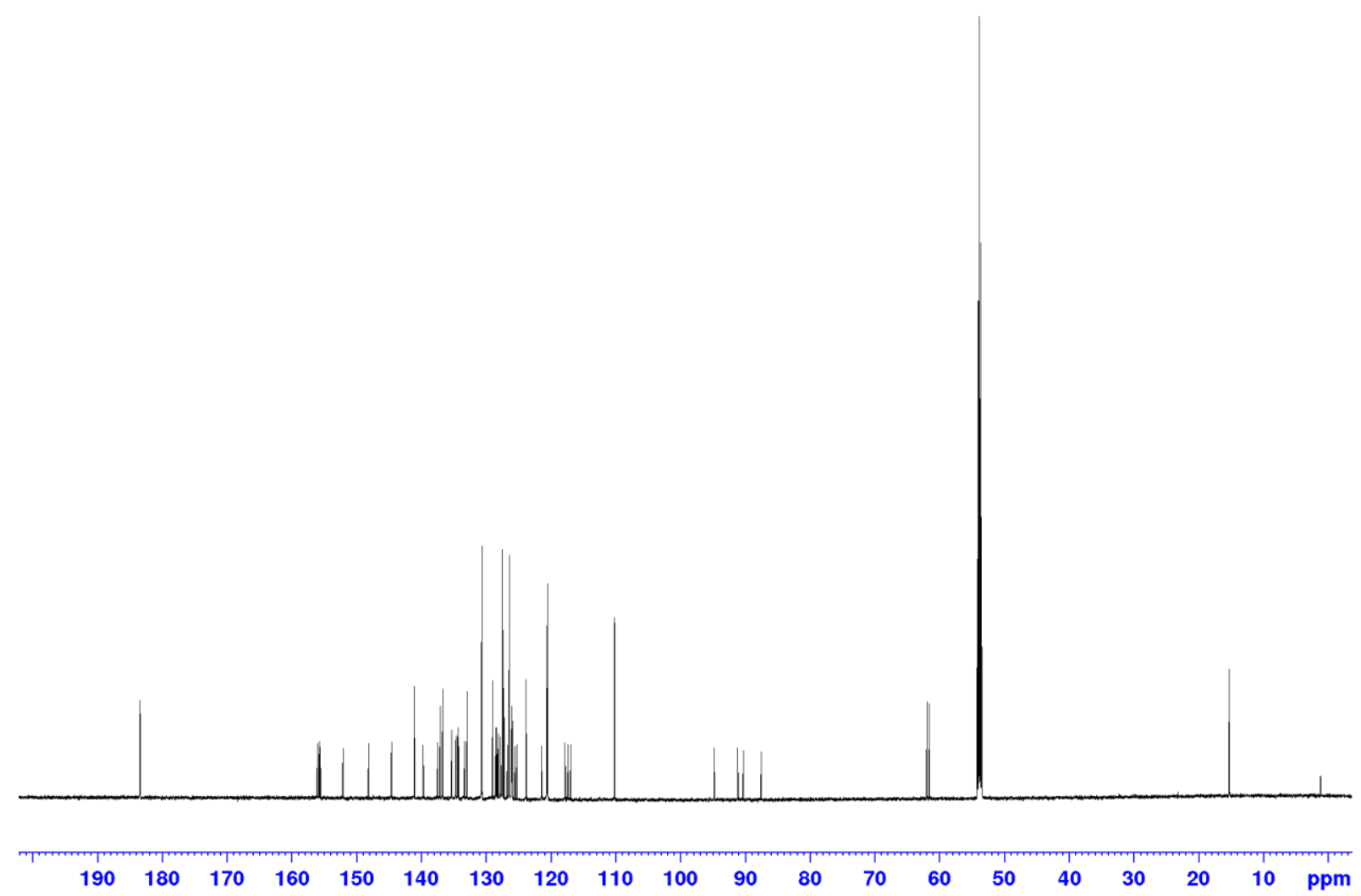

Figure S105. ${ }^{13} \mathrm{C}\left\{{ }^{1} \mathrm{H}\right\}$ NMR of $\mathbf{2 1}(\boldsymbol{S})$ in $\mathrm{CD}_{2} \mathrm{Cl}_{2}$ at a $176.1 \mathrm{MHz}$ spectrometer at $298 \mathrm{~K}$, Overview.

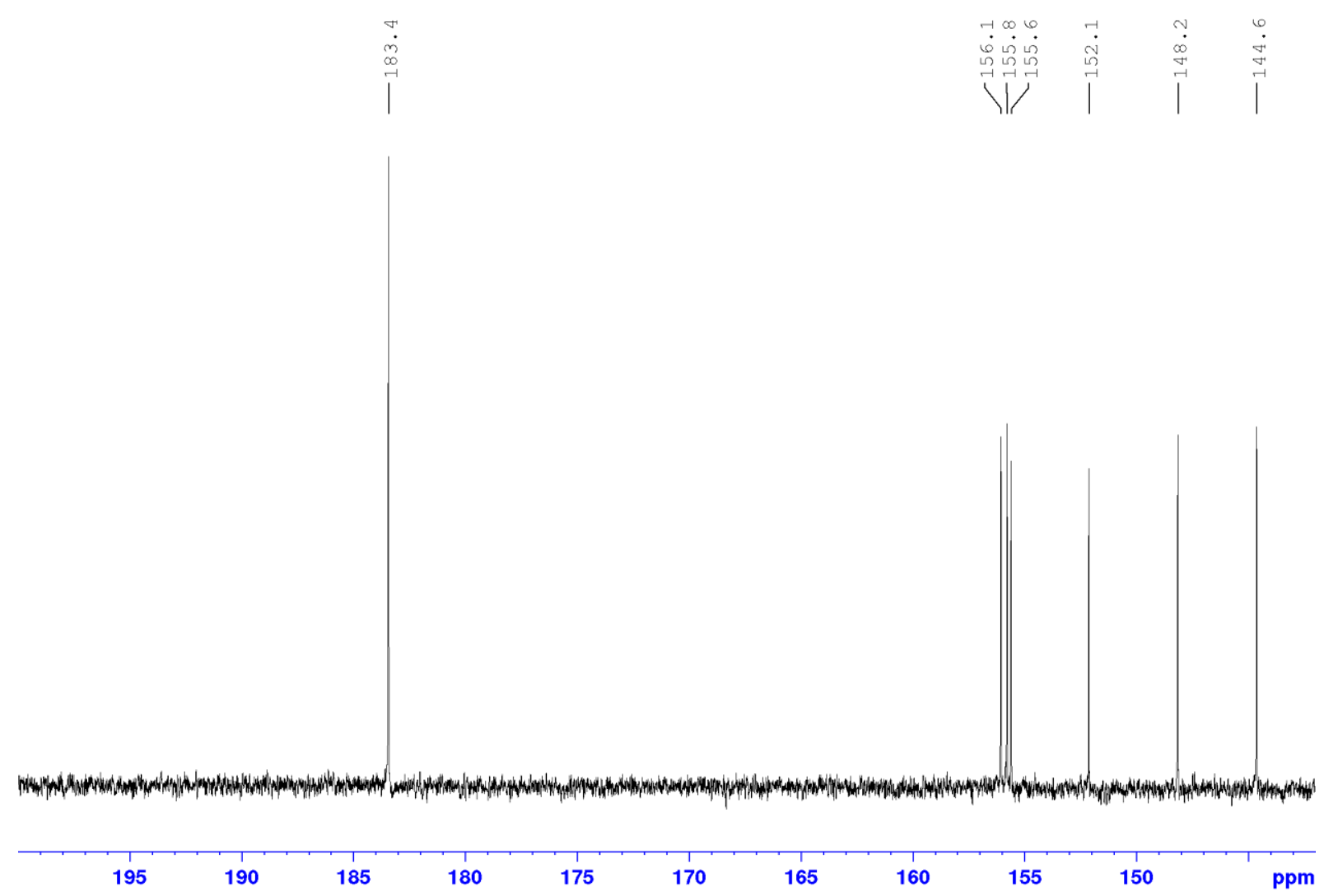

Figure S106. ${ }^{13} \mathrm{C}\left\{{ }^{1} \mathrm{H}\right\}$ NMR of $\mathbf{2 1}(S)$ in $\mathrm{CD}_{2} \mathrm{Cl}_{2}$ at a $176.1 \mathrm{MHz}$ spectrometer at $298 \mathrm{~K}$, Part 1 (200 ppm - 142 ppm). 


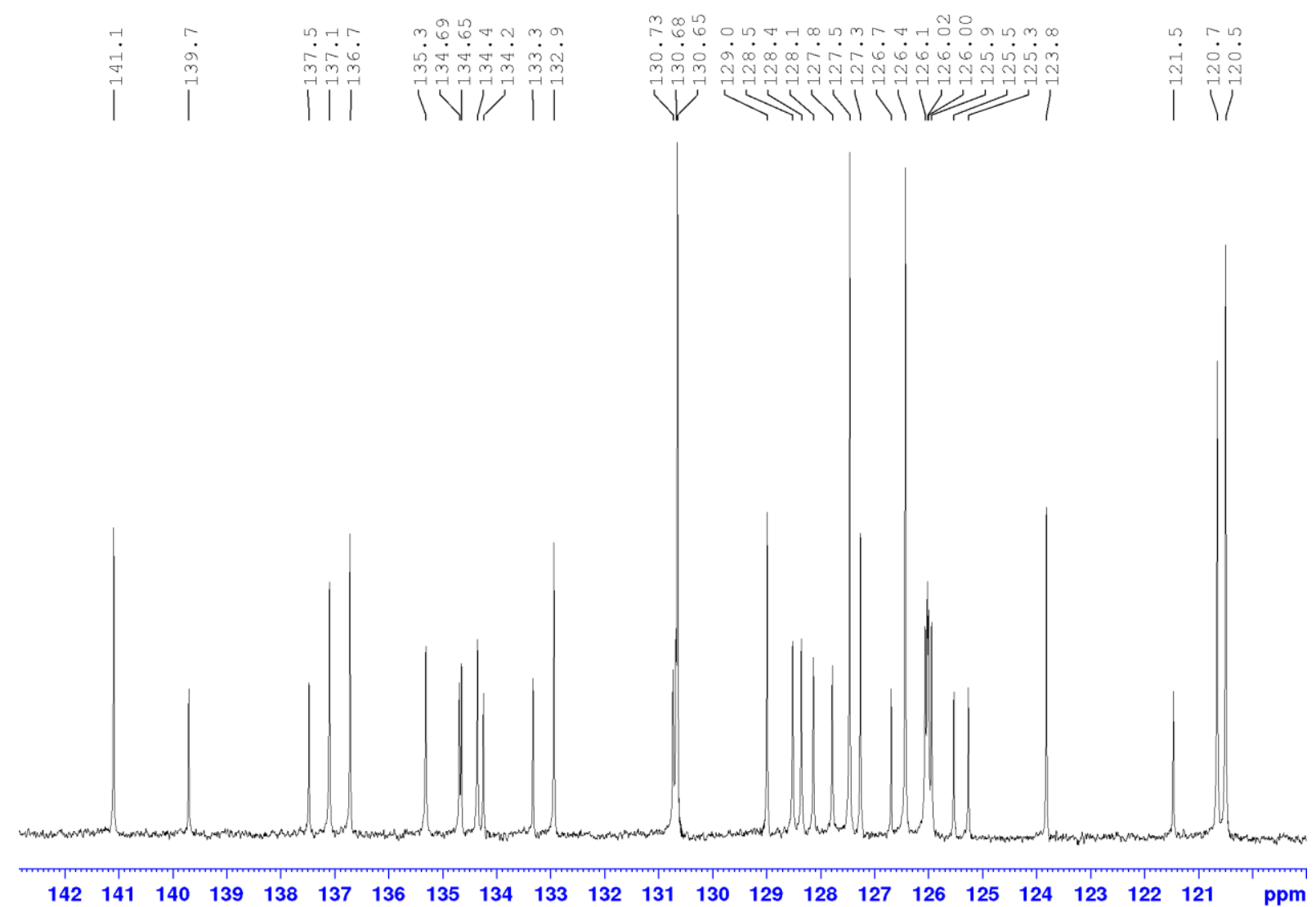

Figure S107. ${ }^{13} \mathrm{C}\left\{{ }^{1} \mathrm{H}\right\}$ NMR of $21(S)$ in $\mathrm{CD}_{2} \mathrm{Cl}_{2}$ at a $176.1 \mathrm{MHz}$ spectrometer at $298 \mathrm{~K}$, Part 2 (142.5 ppm-119 ppm).

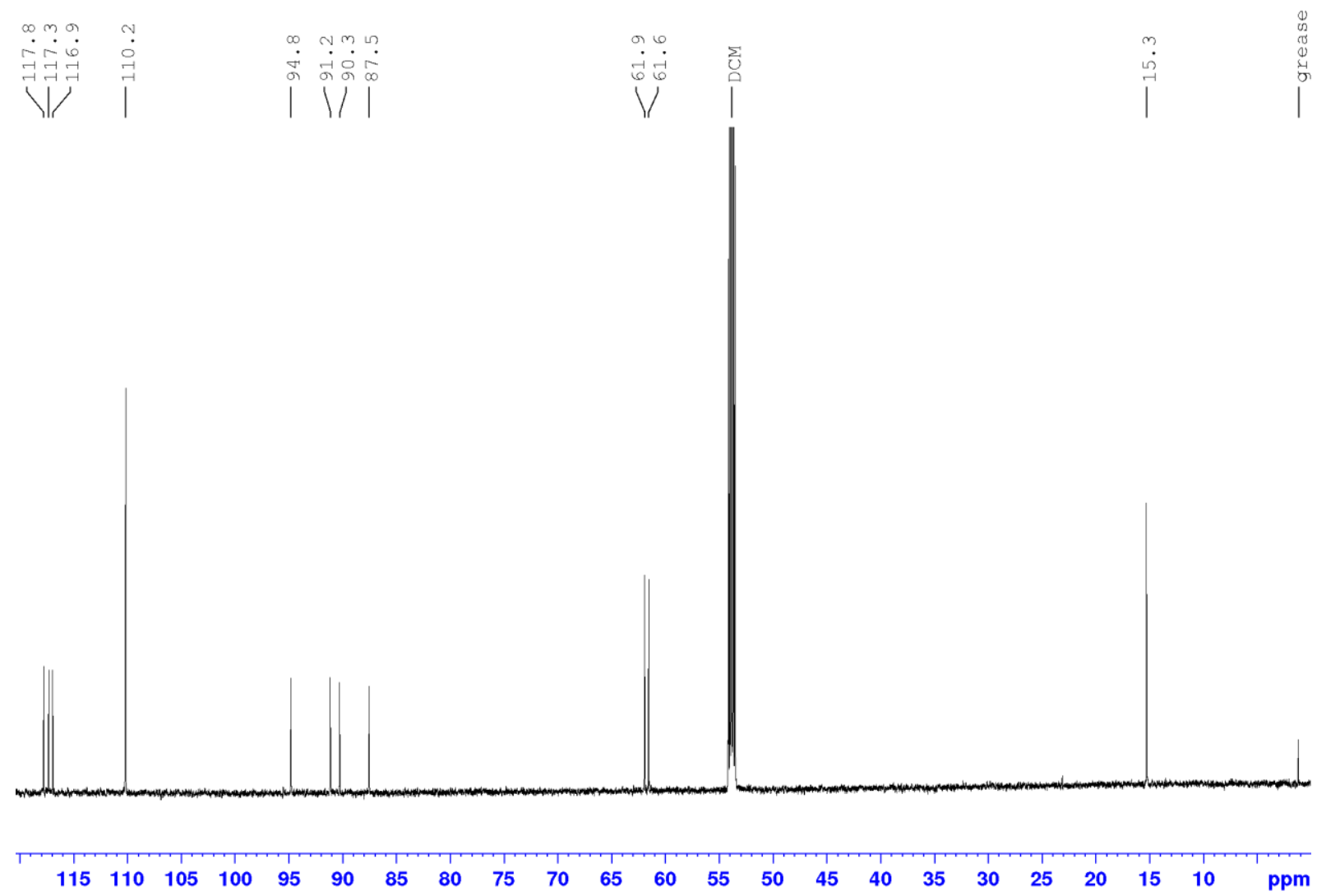

Figure S108. ${ }^{13} \mathrm{C}\left\{{ }^{1} \mathrm{H}\right\}$ NMR of $\mathbf{2 1}(S)$ in $\mathrm{CD}_{2} \mathrm{Cl}_{2}$ at a $176.1 \mathrm{MHz}$ spectrometer at $298 \mathrm{~K}$, Part 3 (120 ppm-0 ppm). 


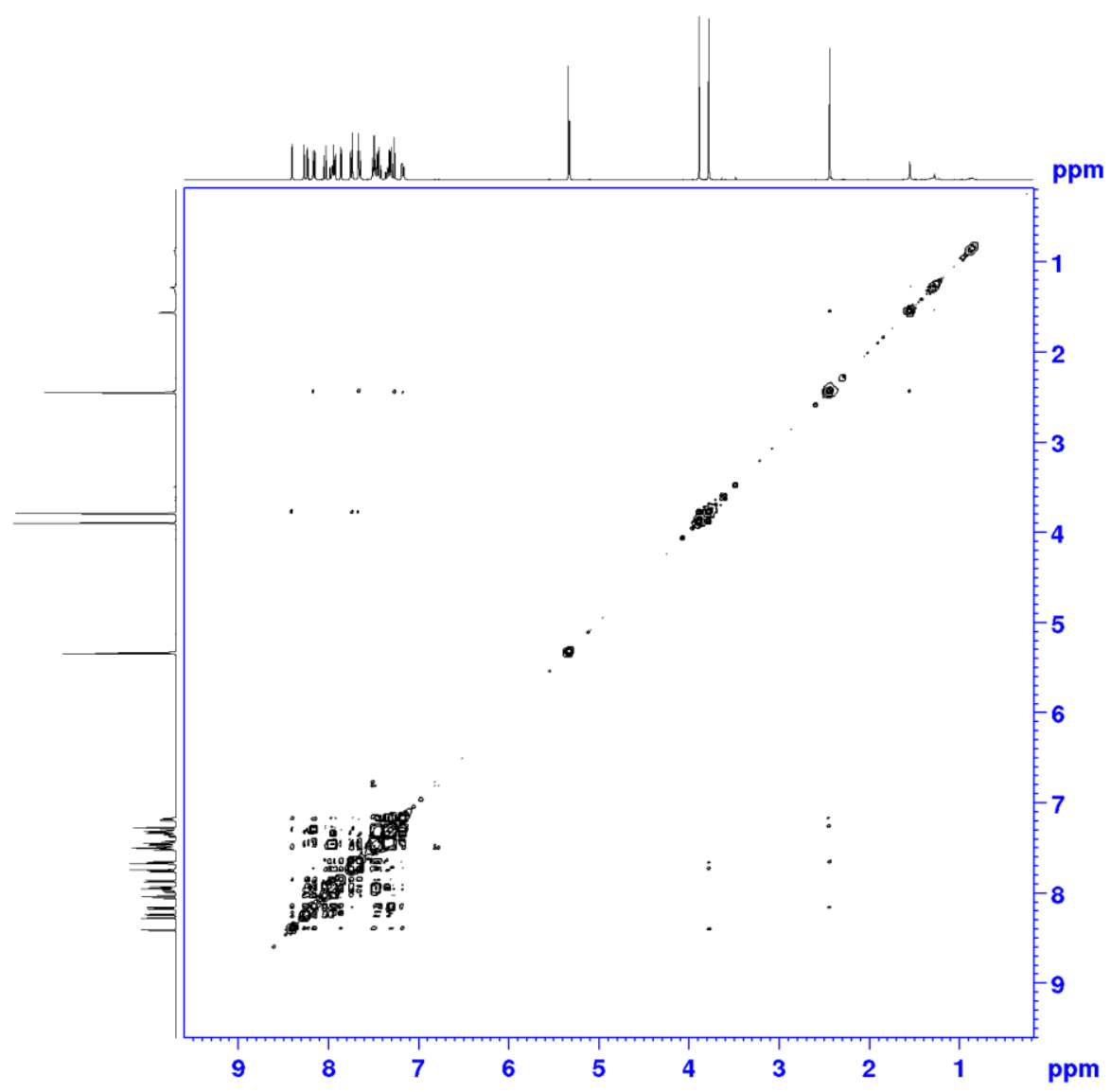

Figure S109. ${ }^{1} \mathrm{H},{ }^{1} \mathrm{H}$ gCOSY NMR spectrum of $\mathbf{2 1}(S)$ in $\mathrm{CD}_{2} \mathrm{Cl}_{2}$ at a $700 \mathrm{MHz}$ spectrometer at $298 \mathrm{~K}$.

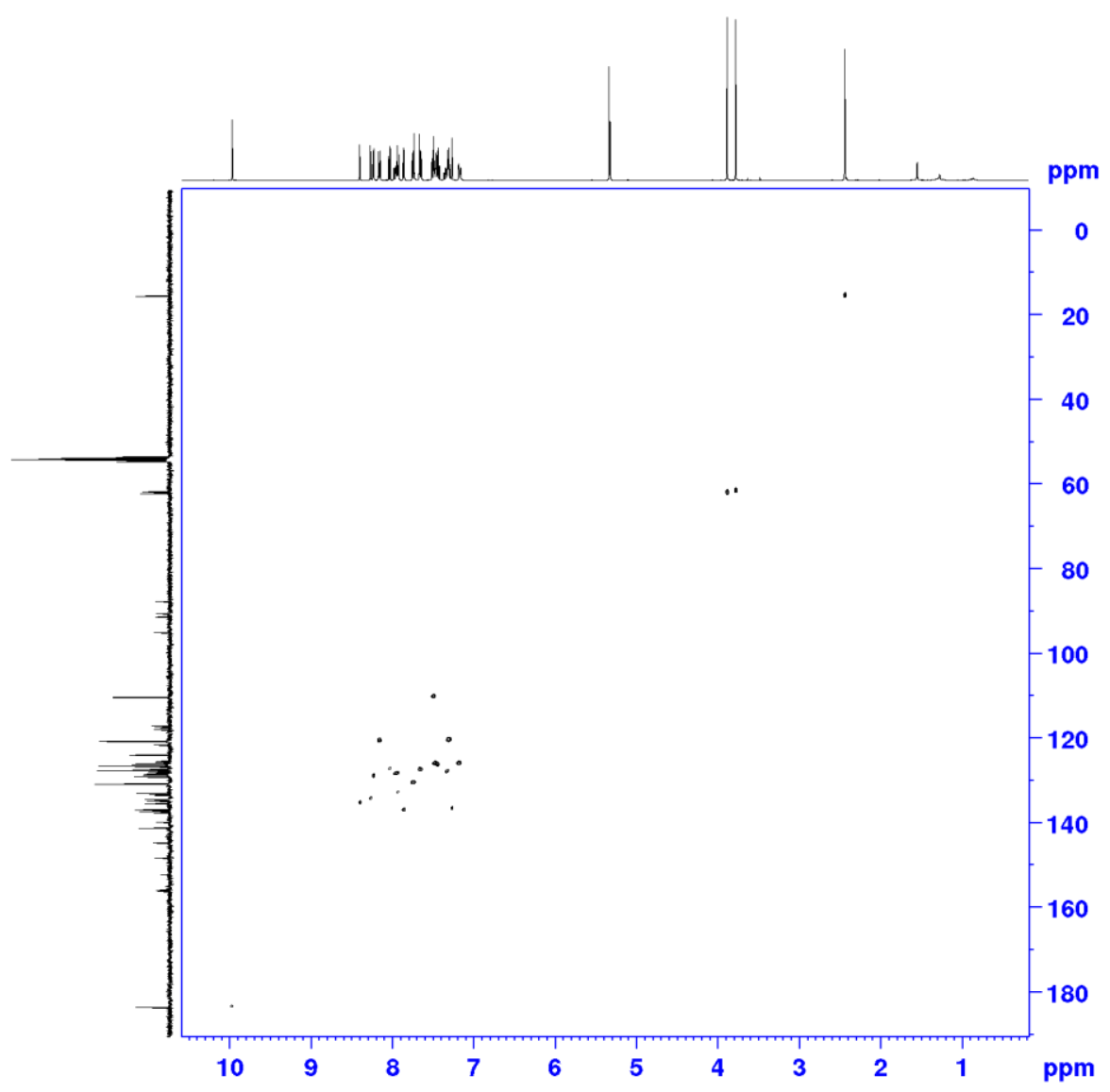

Figure S110. ${ }^{1} \mathrm{H},{ }^{13} \mathrm{C}$ gHSQC NMR spectrum of $\mathbf{2 1}(S)$ in $\mathrm{CD}_{2} \mathrm{Cl}_{2}$ at a $700 \mathrm{MHz}$ spectrometer at $298 \mathrm{~K}$. 


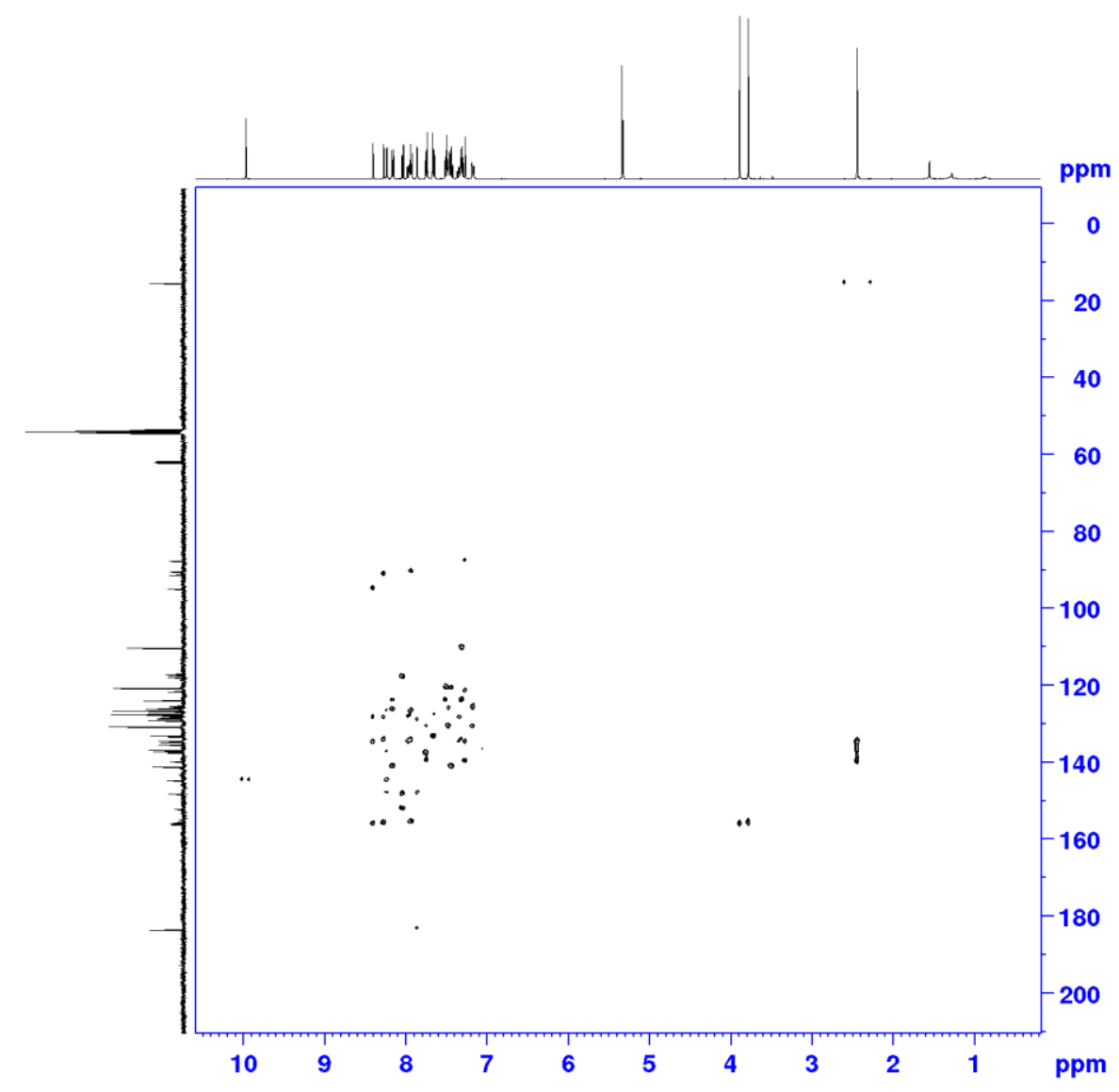

Figure S111. ${ }^{1} \mathrm{H},{ }^{13} \mathrm{C}$ gHMBC NMR spectrum of $21(S)$ in $\mathrm{CD}_{2} \mathrm{Cl}_{2}$ at a $700 \mathrm{MHz}$ spectrometer at $298 \mathrm{~K}$. 

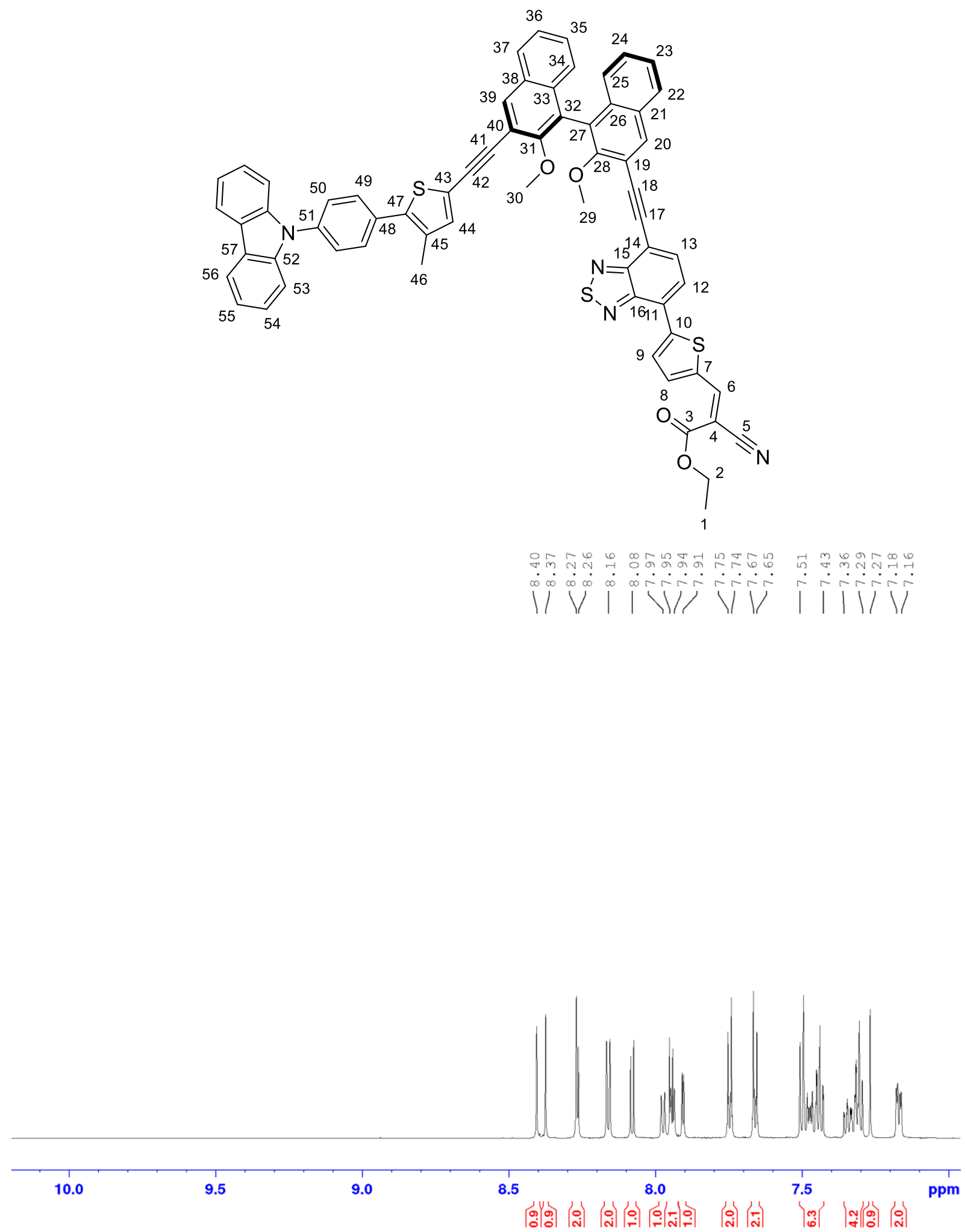

Figure S112. ${ }^{1} \mathrm{H}$ NMR of $\mathbf{2 2}(S)$ in $\mathrm{CD}_{2} \mathrm{Cl}_{2}$ at a $700 \mathrm{MHz}$ spectrometer at $298 \mathrm{~K}$, Part 1 (10.1 ppm - 7.0 ppm). 


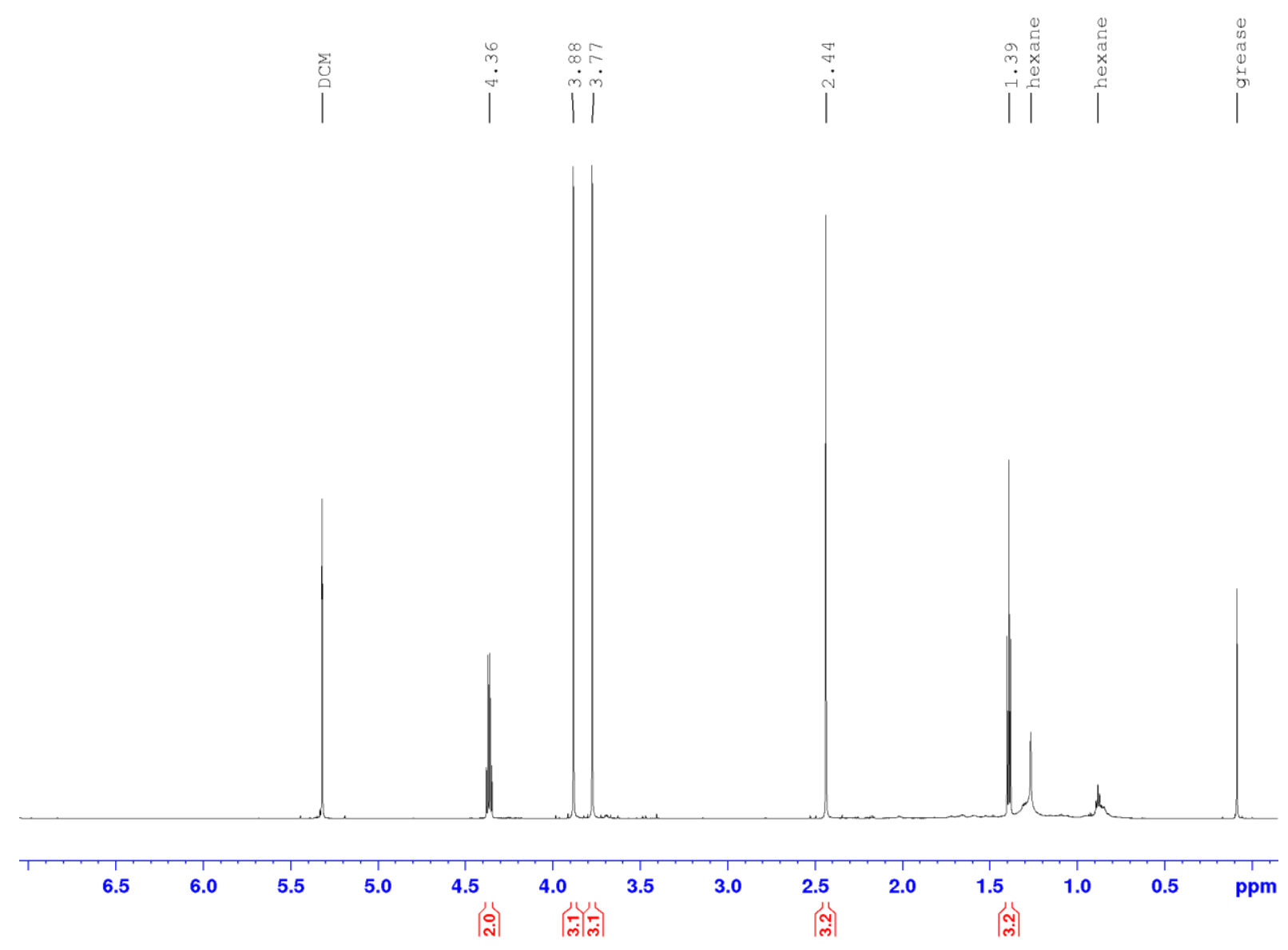

Figure S113. ${ }^{1} \mathrm{H}$ NMR of $\mathbf{2 2}(S)$ in $\mathrm{CD}_{2} \mathrm{Cl}_{2}$ at a $700 \mathrm{MHz}$ spectrometer at $298 \mathrm{~K}$, Part 2 (7.0 ppm - 0.0 ppm).

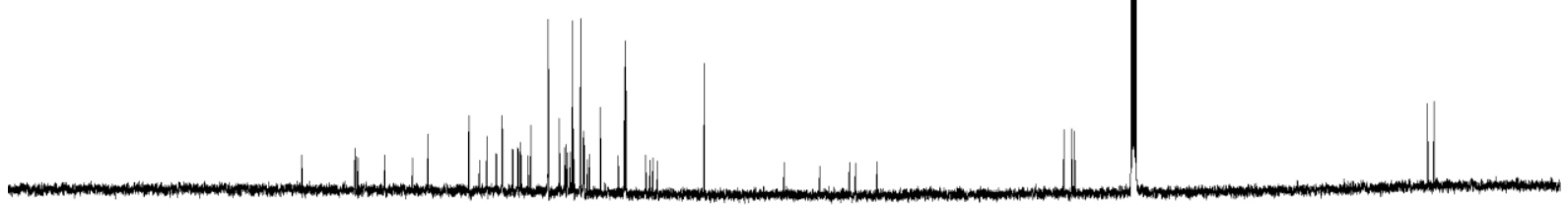

$\begin{array}{lllllllllllllllllllllll}190 & 180 & 170 & 160 & 150 & 140 & 130 & 120 & 110 & 100 & 90 & 80 & 70 & 60 & 50 & 40 & 30 & 20 & 10 & \text { ppm }\end{array}$

Figure S114. ${ }^{13} \mathrm{C}\left\{{ }^{1} \mathrm{H}\right\}$ NMR of $\mathbf{2 2}(S)$ in $\mathrm{CD}_{2} \mathrm{Cl}_{2}$ at a $176.1 \mathrm{MHz}$ spectrometer at $298 \mathrm{~K}$, Overview. 


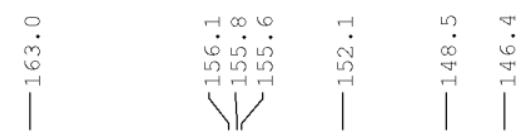

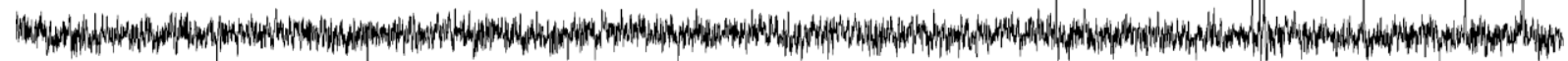

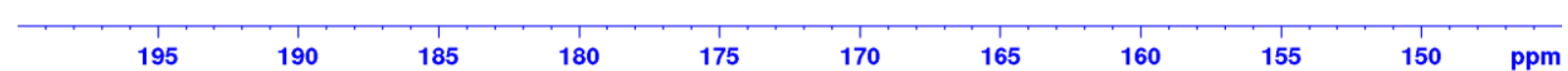

Figure S115. ${ }^{13} \mathrm{C}\left\{{ }^{1} \mathrm{H}\right\}$ NMR of $\mathbf{2 2}(S)$ in $\mathrm{CD}_{2} \mathrm{Cl}_{2}$ at a $176.1 \mathrm{MHz}$ spectrometer at $298 \mathrm{~K}$, Part 1 (190 ppm - 145 ppm).
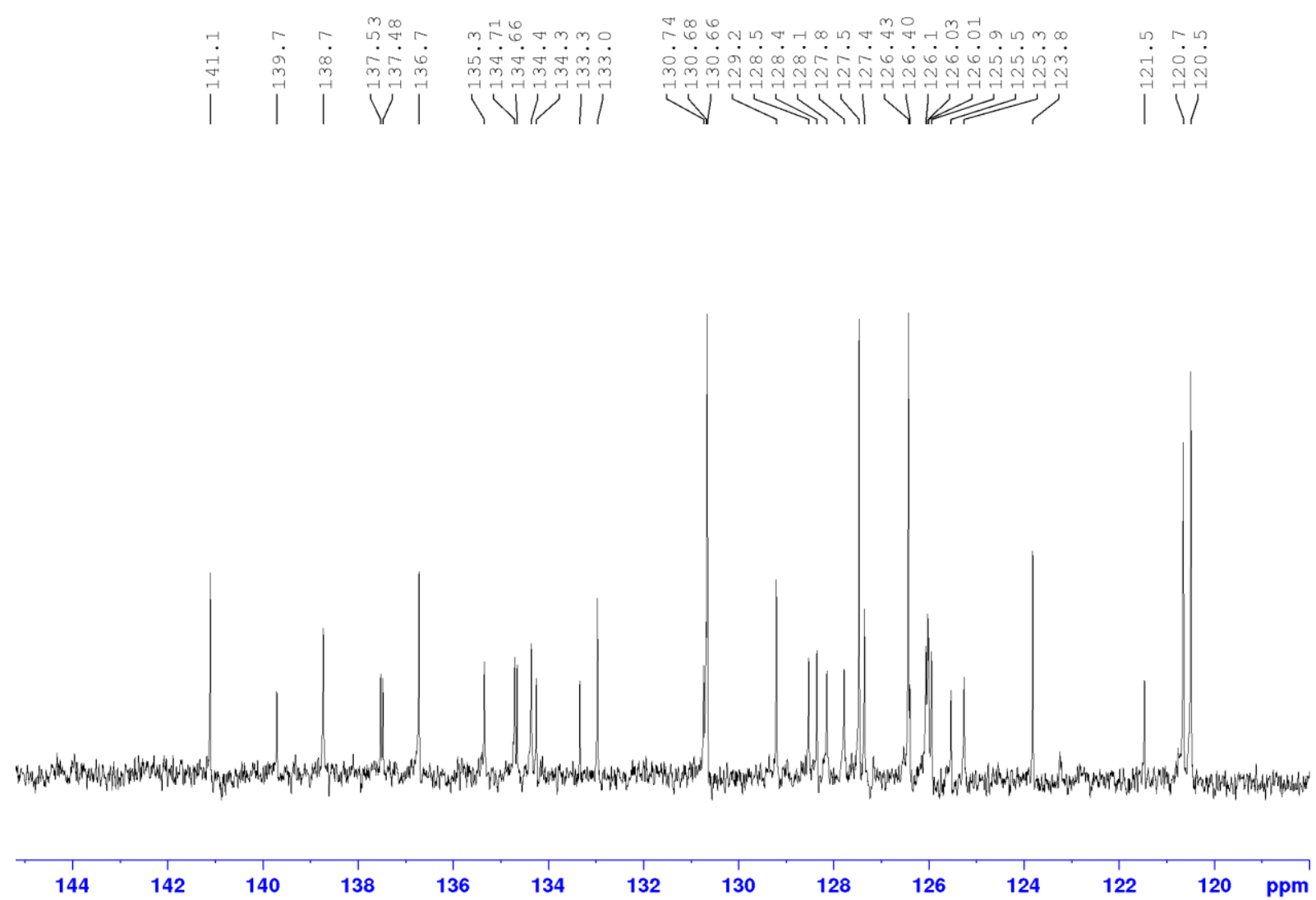

Figure S116. ${ }^{13} \mathrm{C}$ NMR of $22(S)$ in $\mathrm{CD}_{2} \mathrm{Cl}_{2}$ at a $176.1 \mathrm{MHz}$ spectrometer at $298 \mathrm{~K}$, Part 2 (145 ppm-118 ppm). 


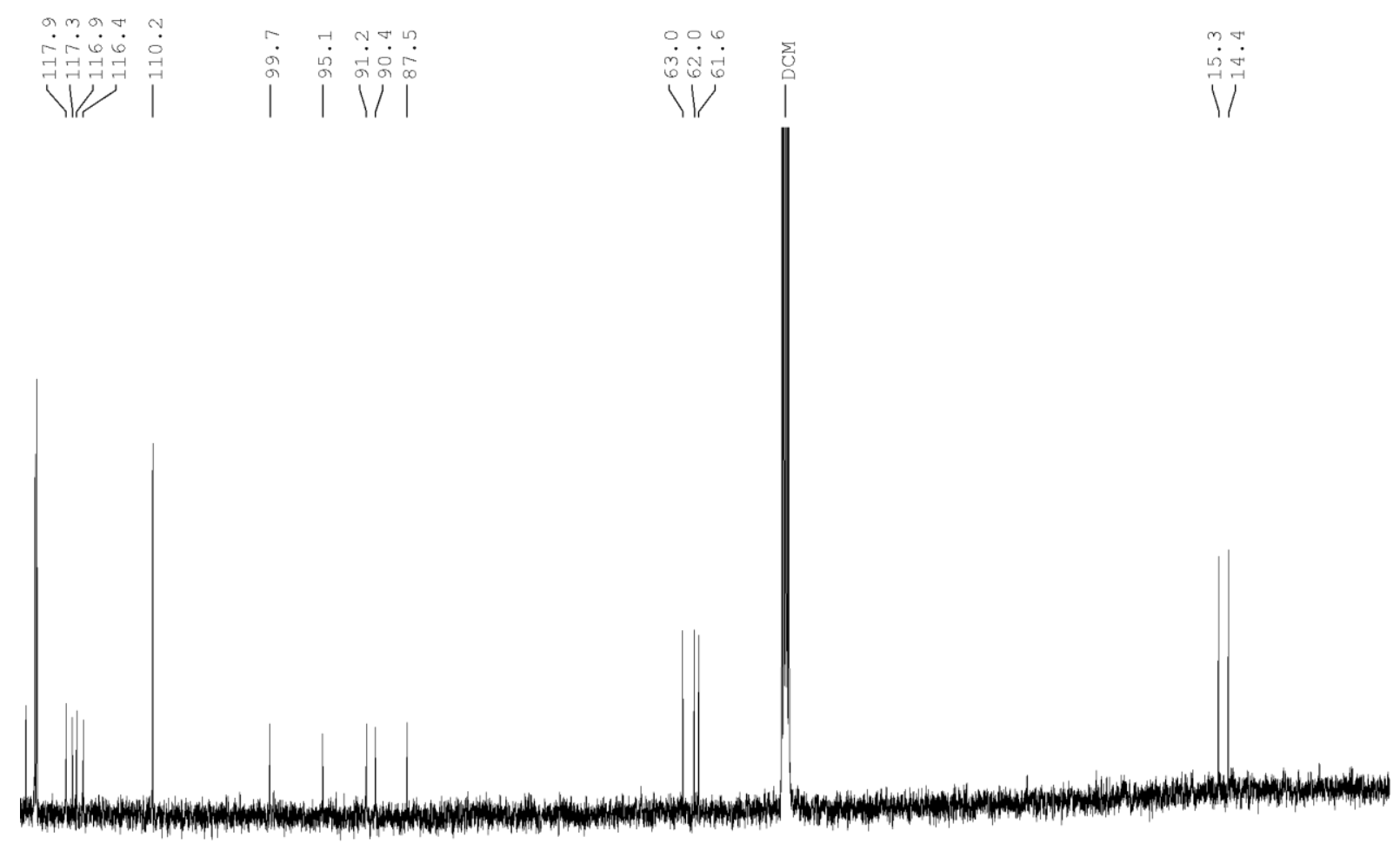

$\begin{array}{llllllllllllllllllllllll}120 & 115 & 110 & 105 & 100 & 95 & 90 & 85 & 80 & 75 & 70 & 65 & 60 & 55 & 50 & 45 & 40 & 35 & 30 & 25 & 20 & 15 & 10 & \mathrm{ppm}\end{array}$

Figure S117. ${ }^{13} \mathrm{C}$ NMR of $\mathbf{2 2}(\mathrm{S})$ in $\mathrm{CD}_{2} \mathrm{Cl}_{2}$ at a $176.1 \mathrm{MHz}$ spectrometer at $298 \mathrm{~K}$, Part 3 (122 ppm-0 ppm). 


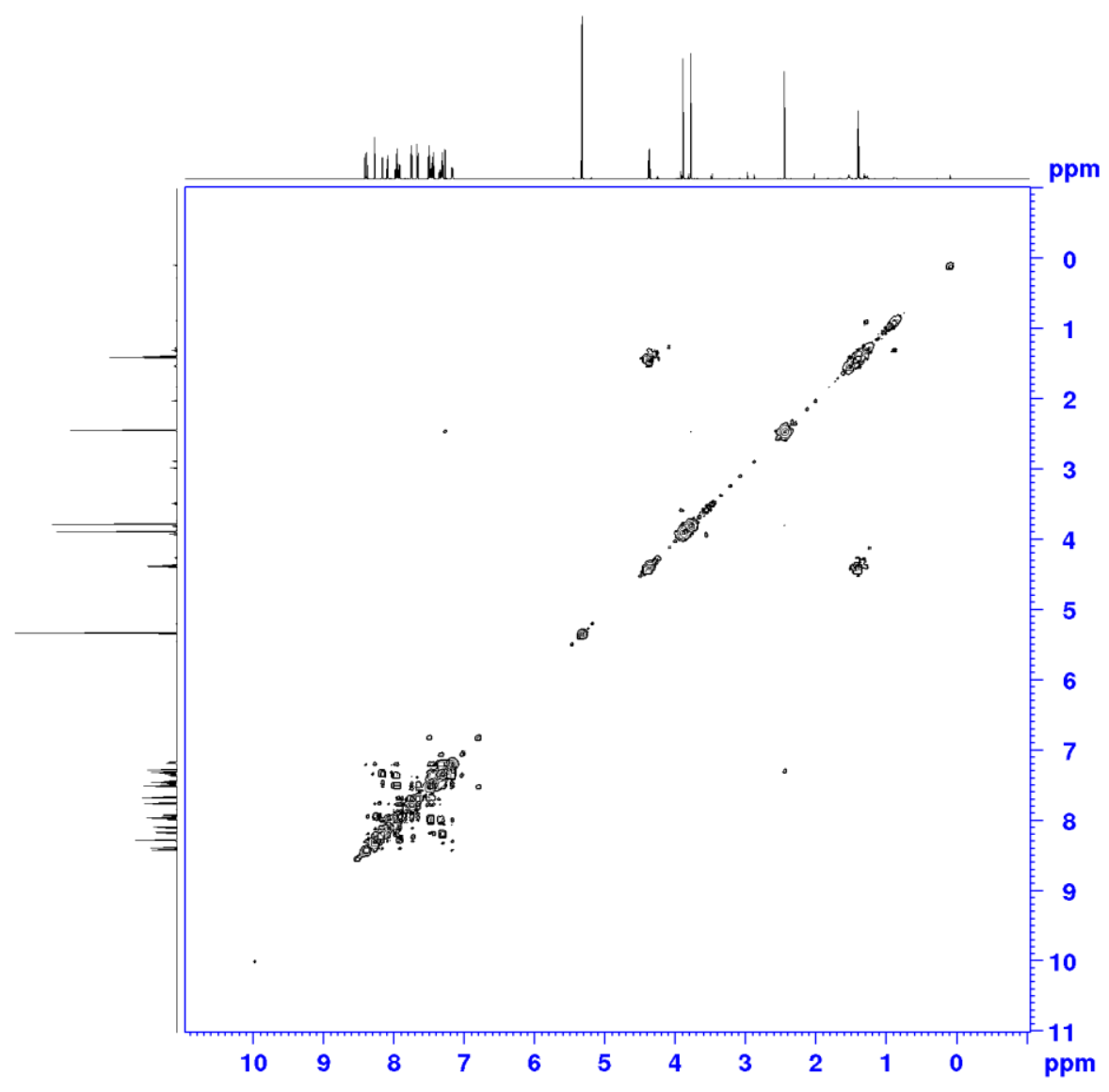

Figure S118. ${ }^{1} \mathrm{H},{ }^{1} \mathrm{H}$ gCOSY NMR spectrum of $\mathbf{2 2}(S)$ in $\mathrm{CD}_{2} \mathrm{Cl}_{2}$ at a $700 \mathrm{MHz}$ spectrometer at $298 \mathrm{~K}$.

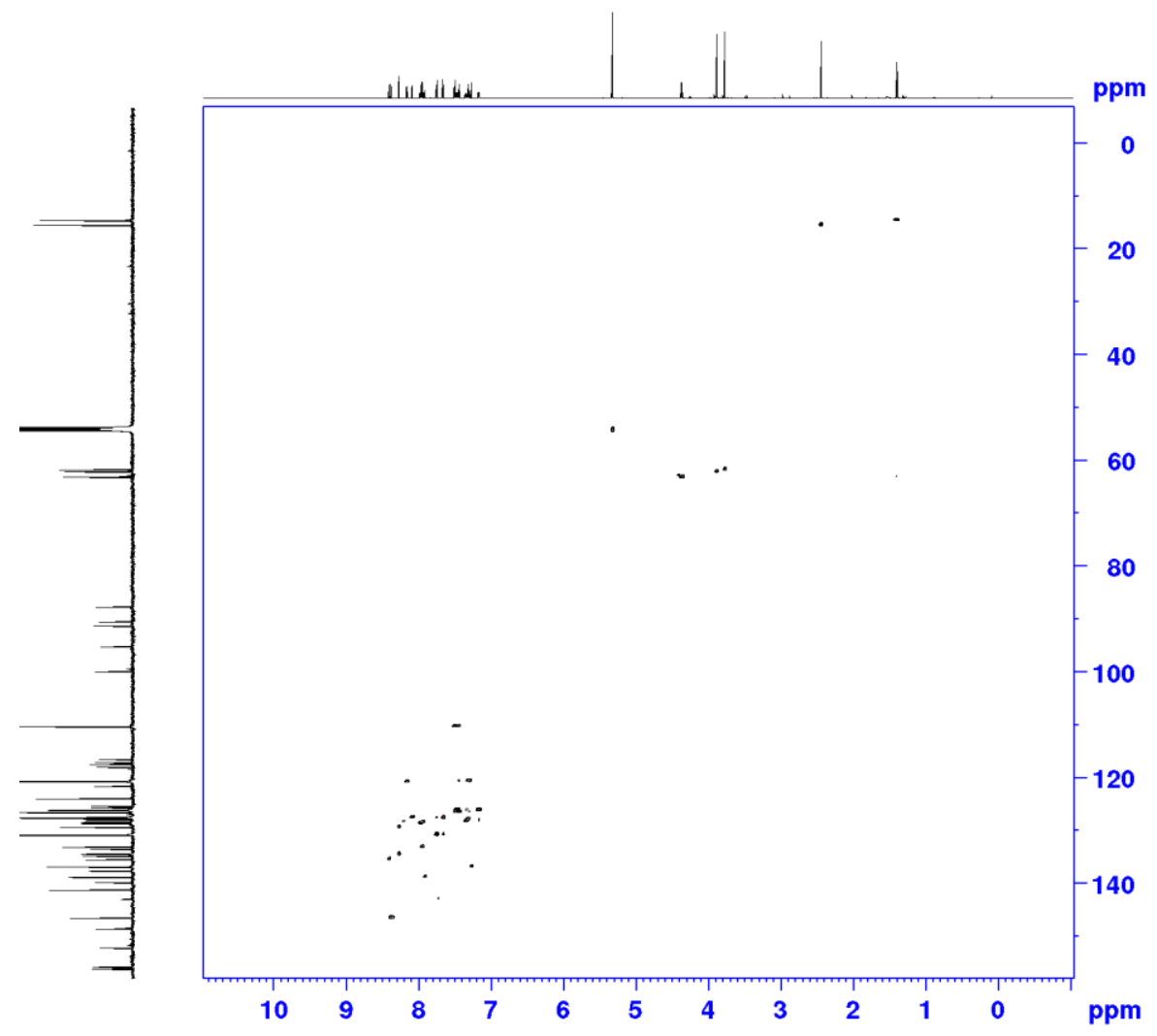

Figure S119. ${ }^{1} \mathrm{H},{ }^{13} \mathrm{C}$ gHSQC NMR spectrum of $22(\mathbf{S})$ in $\mathrm{CD}_{2} \mathrm{Cl}_{2}$ at a $700 \mathrm{MHz}$ spectrometer at $298 \mathrm{~K}$. 


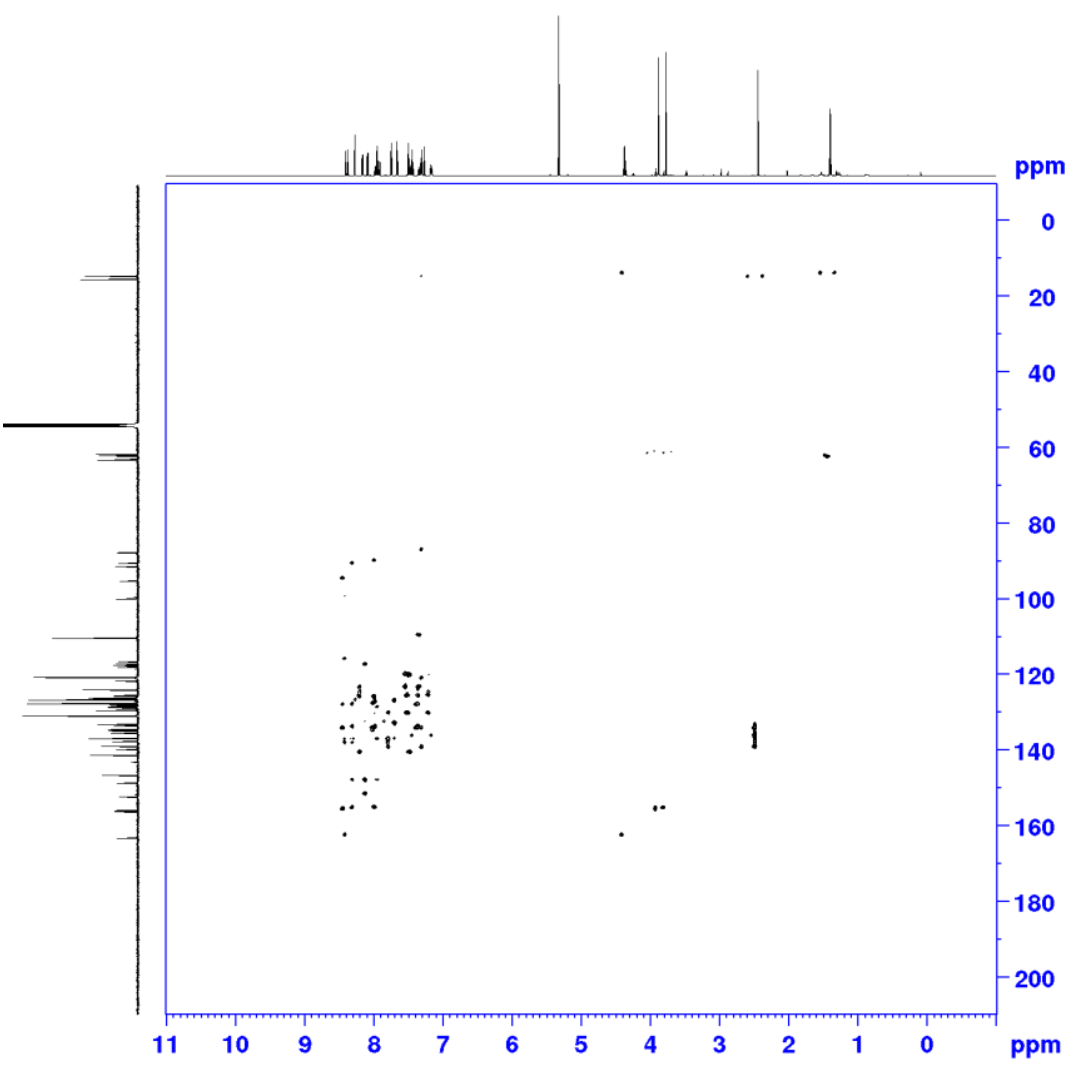

Figure S120. ${ }^{1} \mathrm{H},{ }^{13} \mathrm{C}$ gHMBC NMR spectrum of $22(S)$ in $\mathrm{CD}_{2} \mathrm{Cl}_{2}$ at a $700 \mathrm{MHz}$ spectrometer at $298 \mathrm{~K}$.

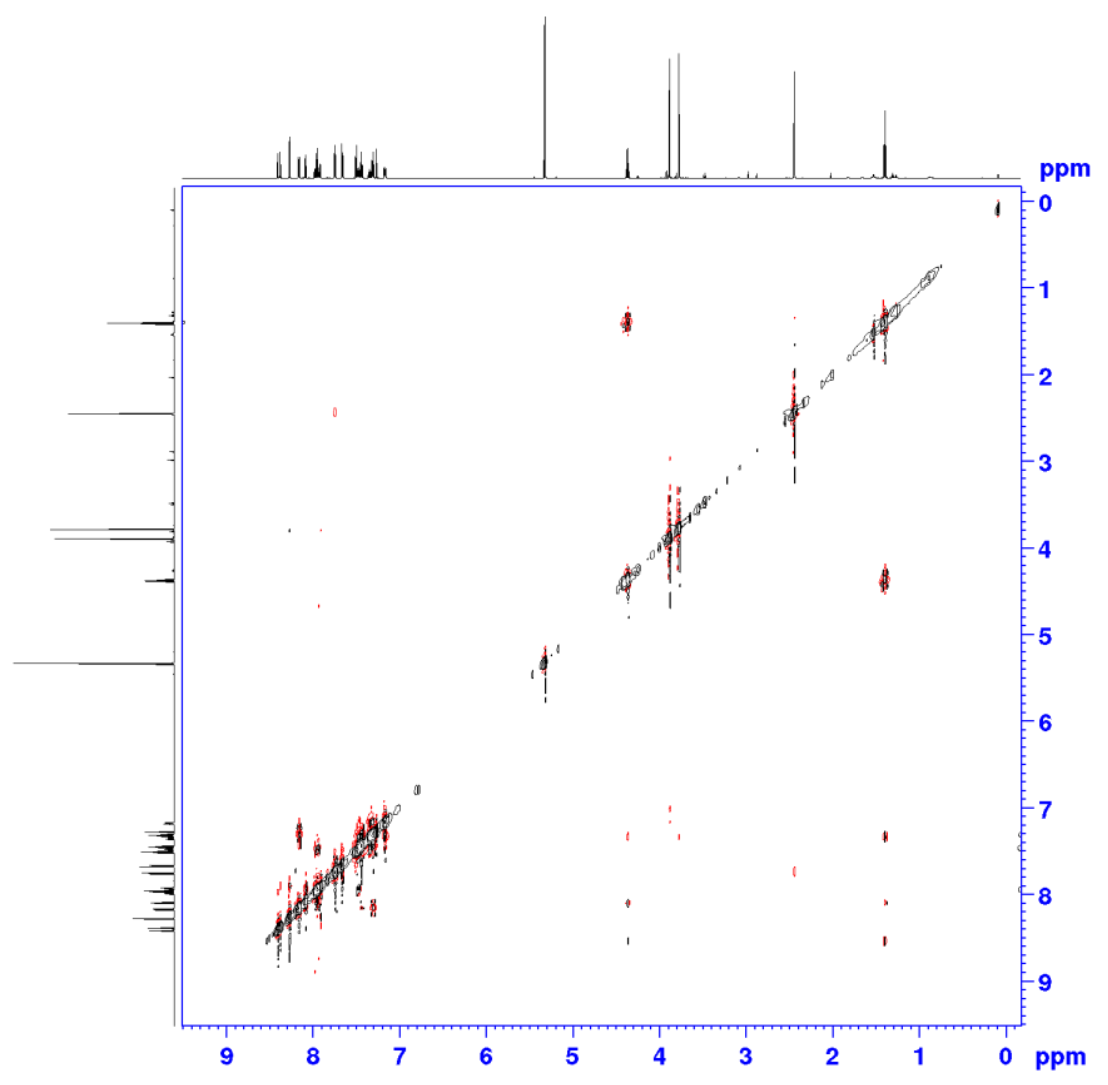

Figure S121. ${ }^{1} \mathrm{H},{ }^{1} \mathrm{H}$ gNOESY NMR spectrum of $\mathbf{2 2}(\boldsymbol{S})$ in $\mathrm{CD}_{2} \mathrm{Cl}_{2}$ at a $700 \mathrm{MHz}$ spectrometer at $298 \mathrm{~K}$. 


\section{UV-Vis- and Fluorescence Spectra}

The fluorescence spectra were scaled to the UV-Vis spectra (absorption coefficients and quantum yields are assigned in Table S1).

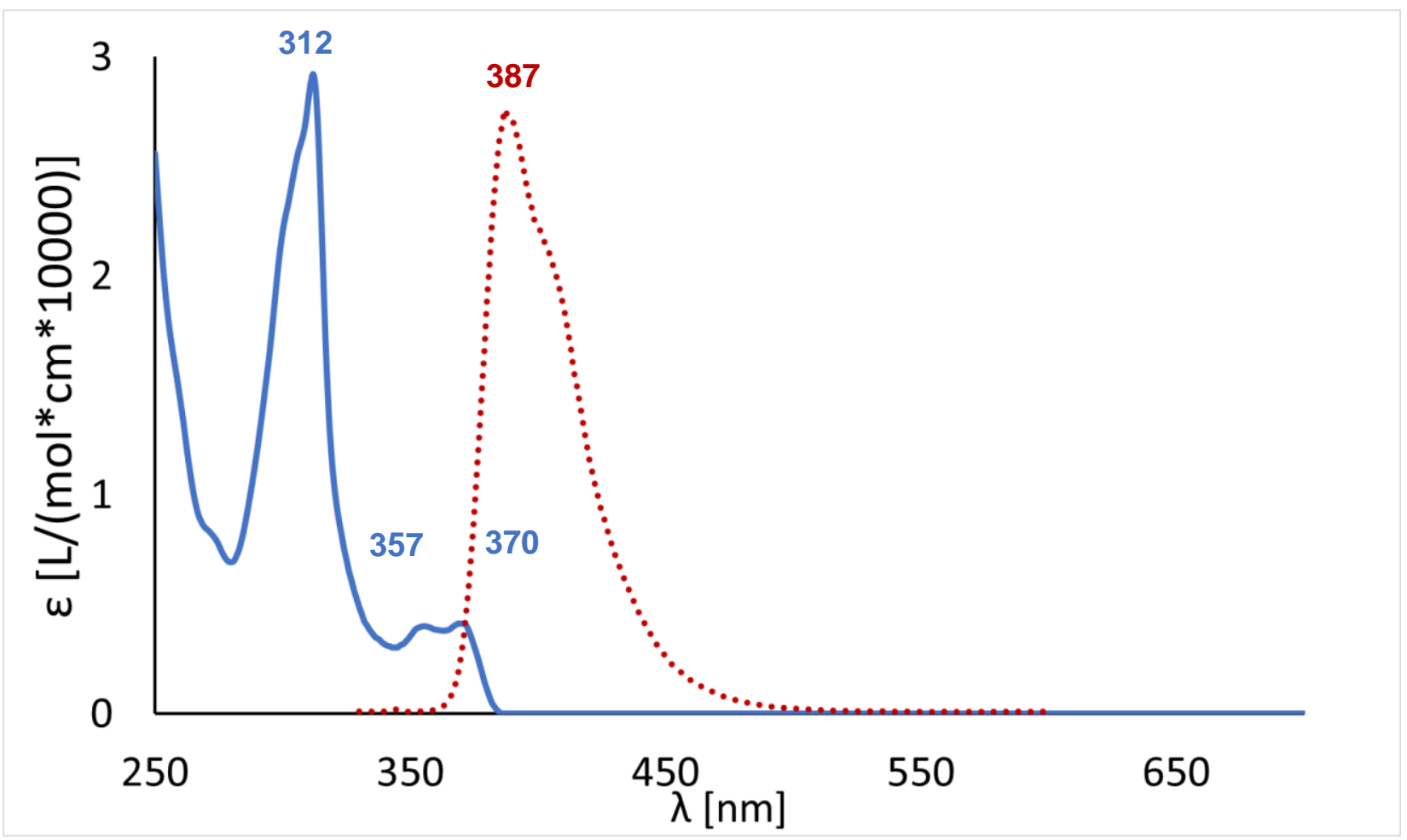

Figure S122. UV-Vis (blue line)- and Fluorescence (red dotted line) spectra of $\mathbf{2 b}$ in DCM.

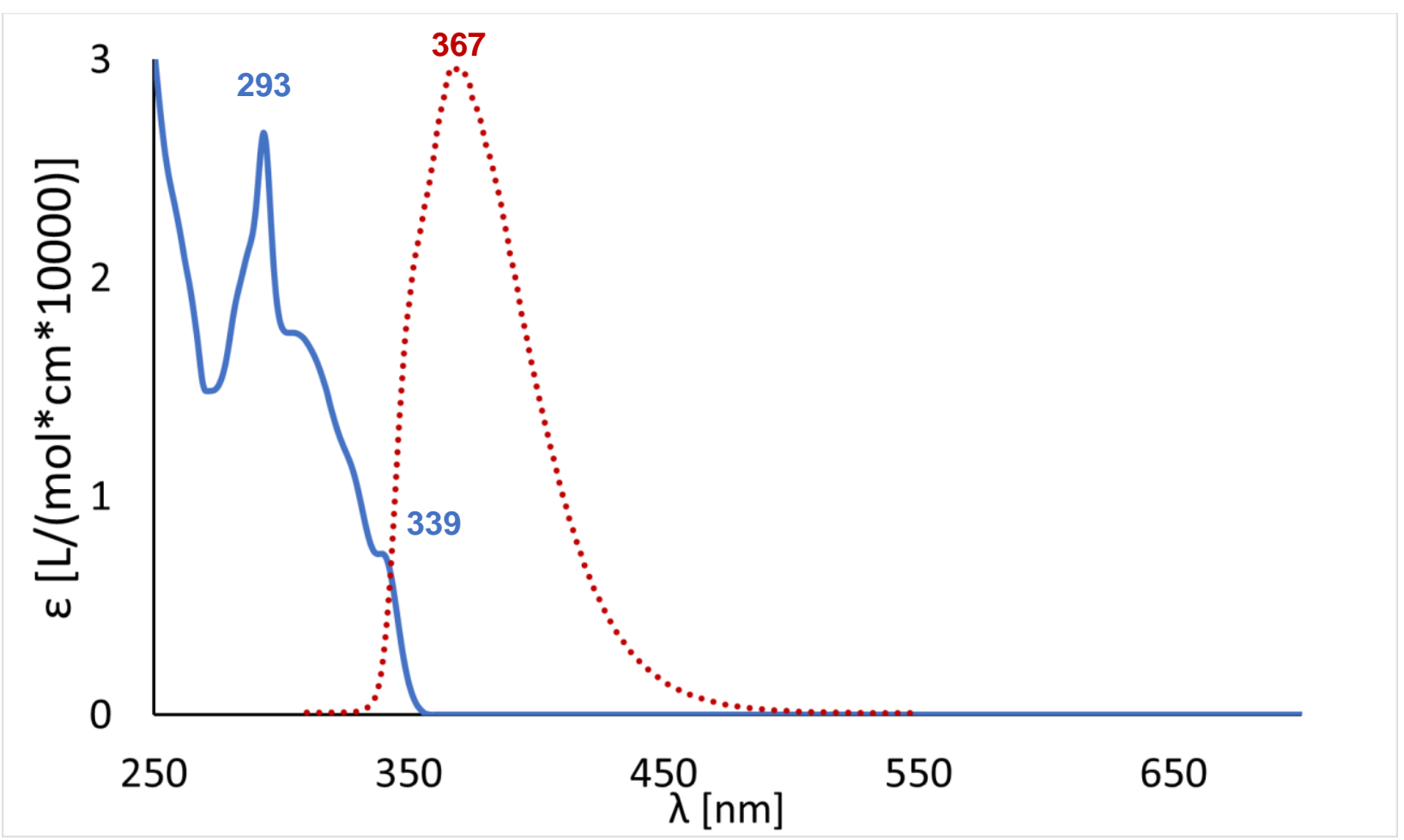

Figure S123. UV-Vis (blue line)- and Fluorescence (red dotted line) spectra of 4a in DCM. 


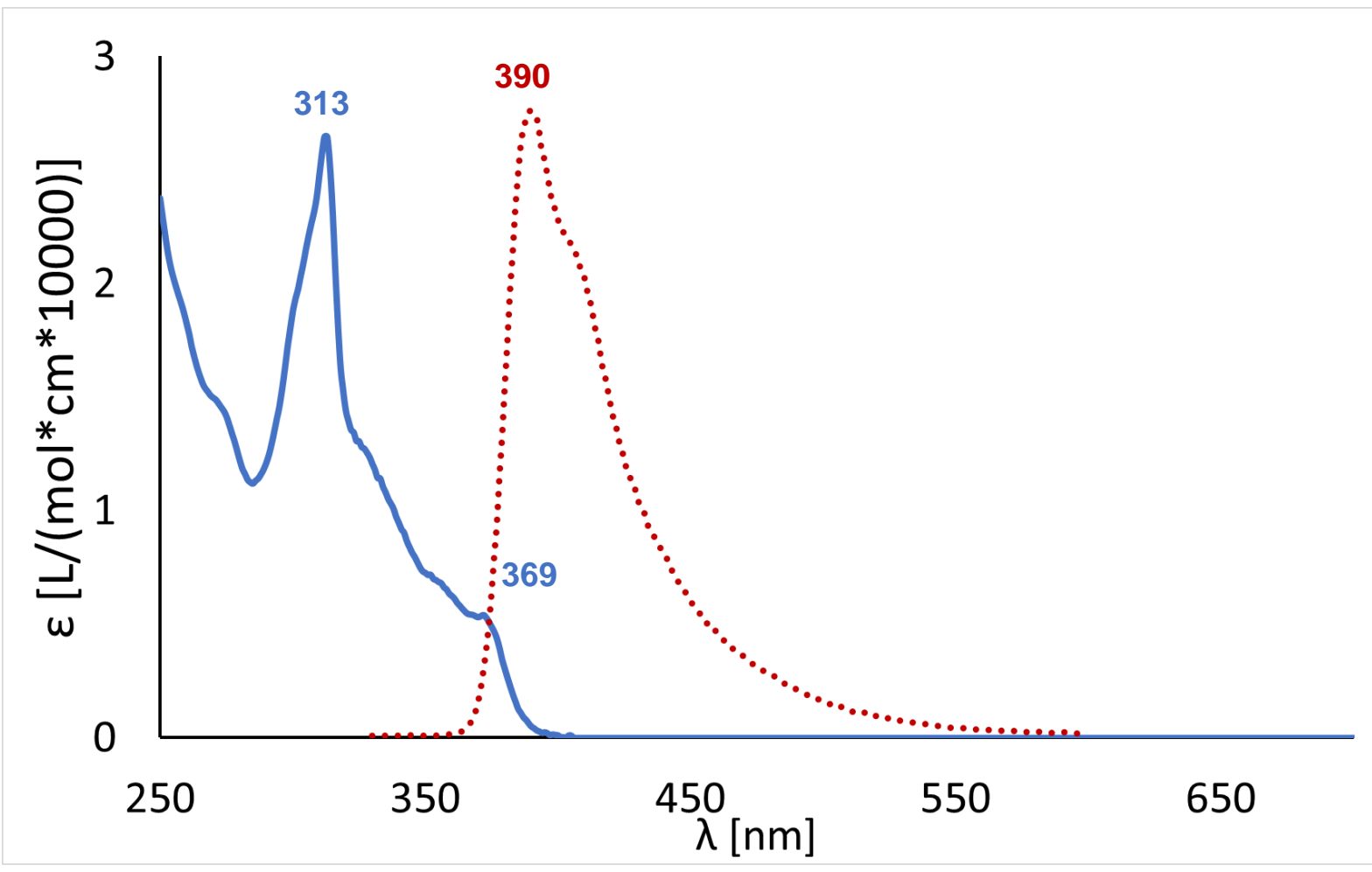

Figure S124. UV-Vis (blue line)- and Fluorescence (red dotted line) spectra of $\mathbf{4 b}$ in DCM.

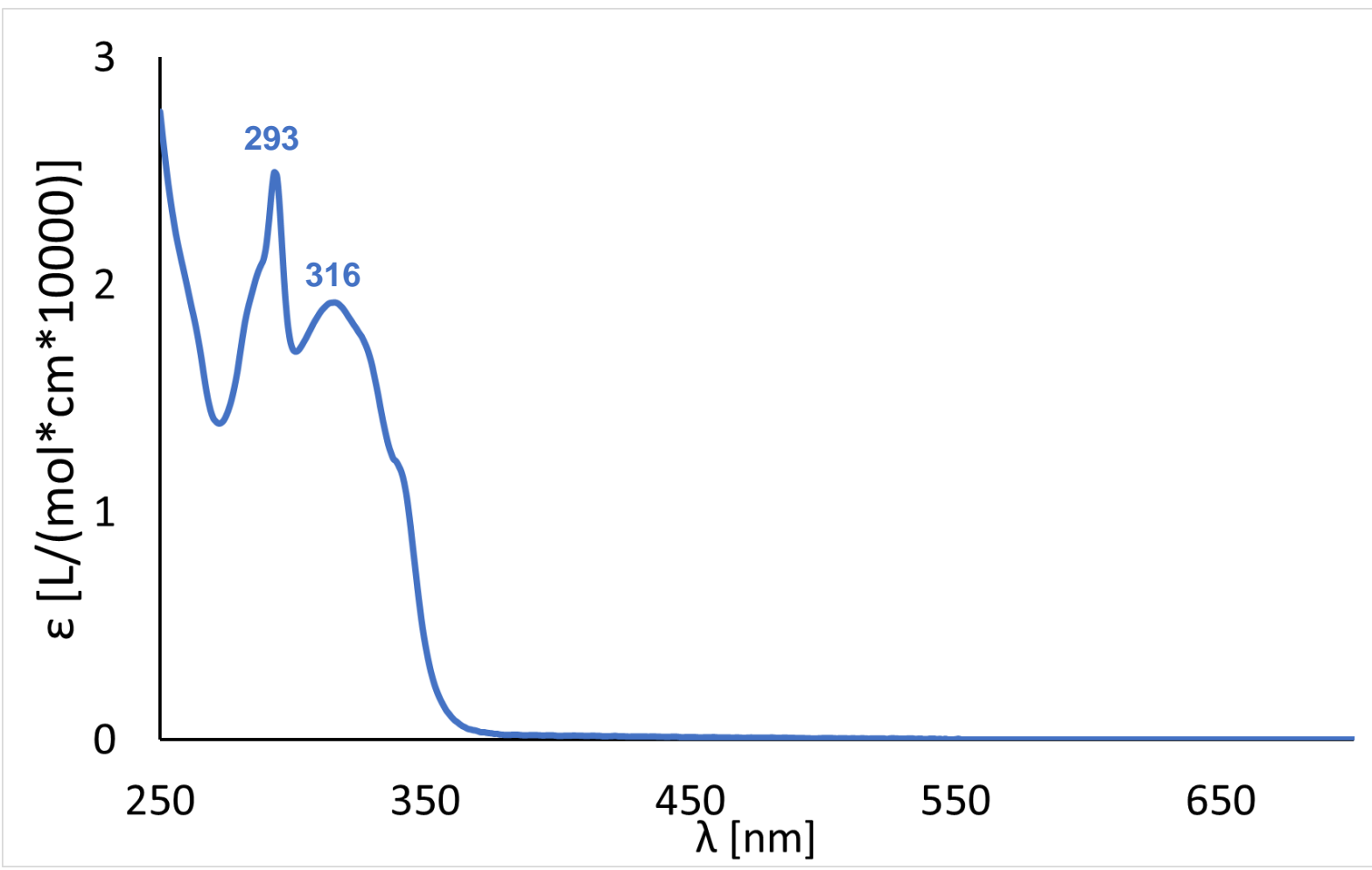

Figure S125. UV-Vis spectra of 5a in DCM. 


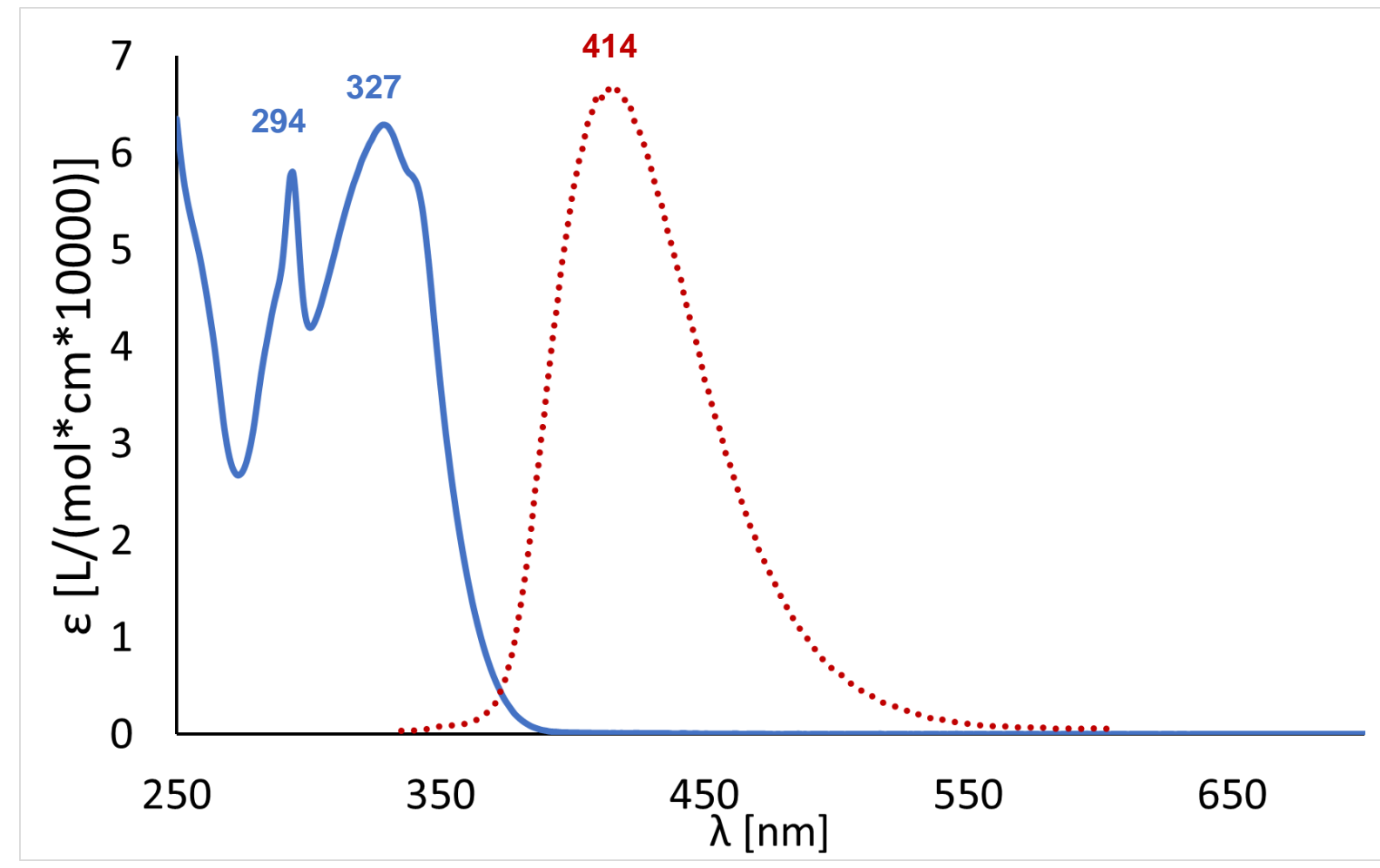

Figure S126. UV-Vis (blue line)- and Fluorescence (red dotted line) spectra of 6 in DCM.

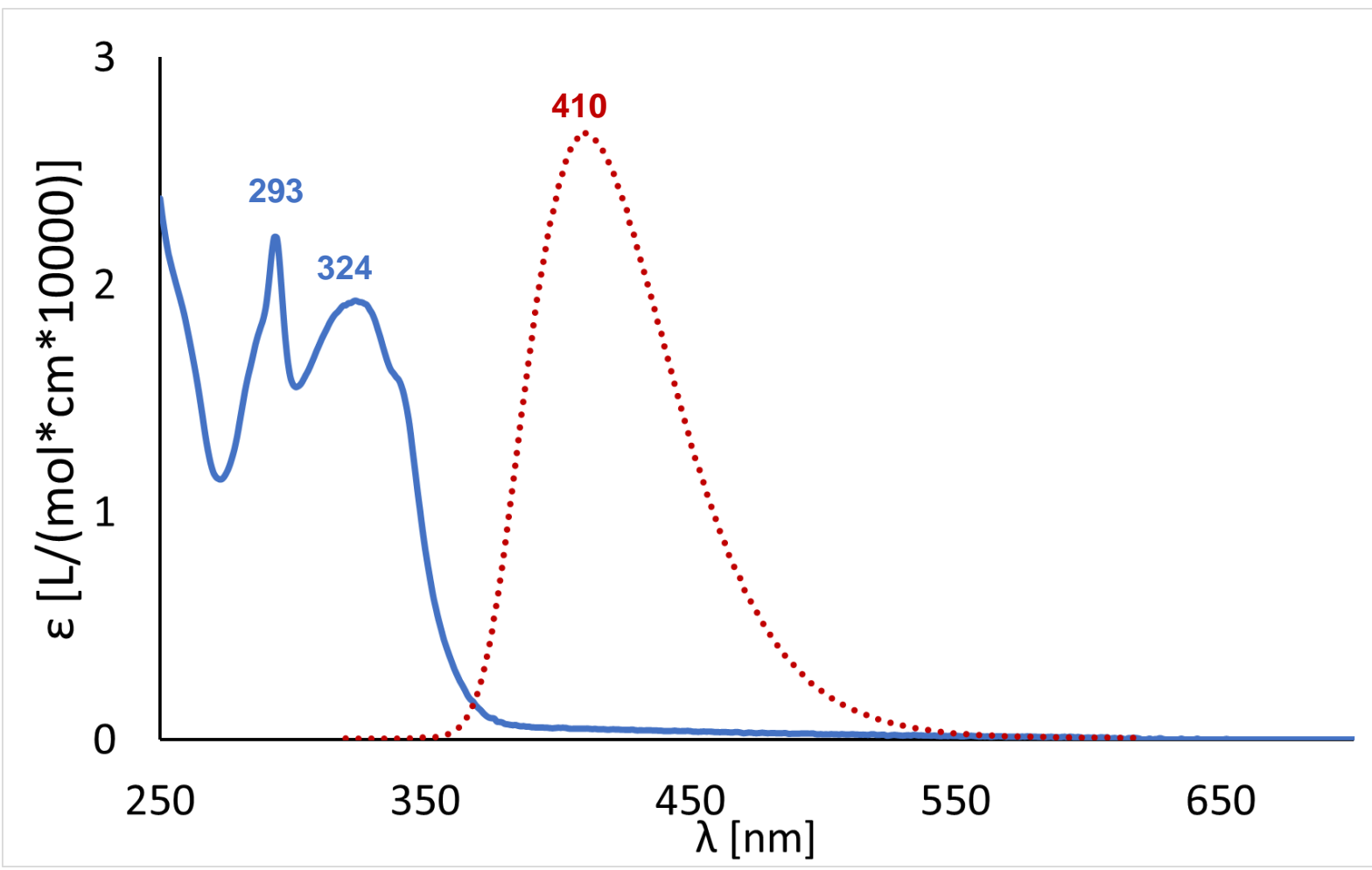

Figure S127. UV-Vis (blue line)- and Fluorescence (red dotted line) spectra of 7 in DCM. 


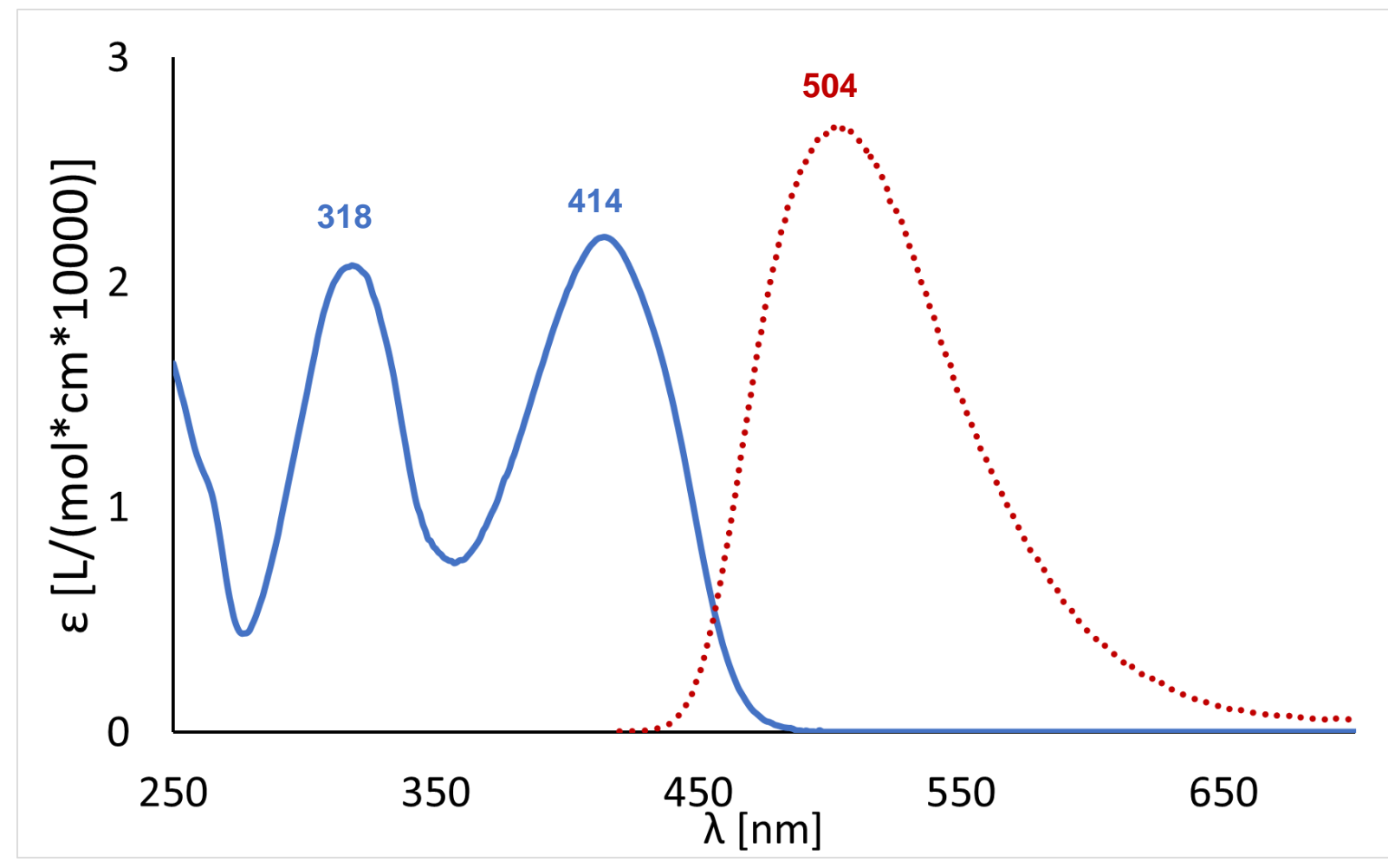

Figure S128. UV-Vis (blue line)- and Fluorescence (red dotted line) spectra of 9 in DCM.

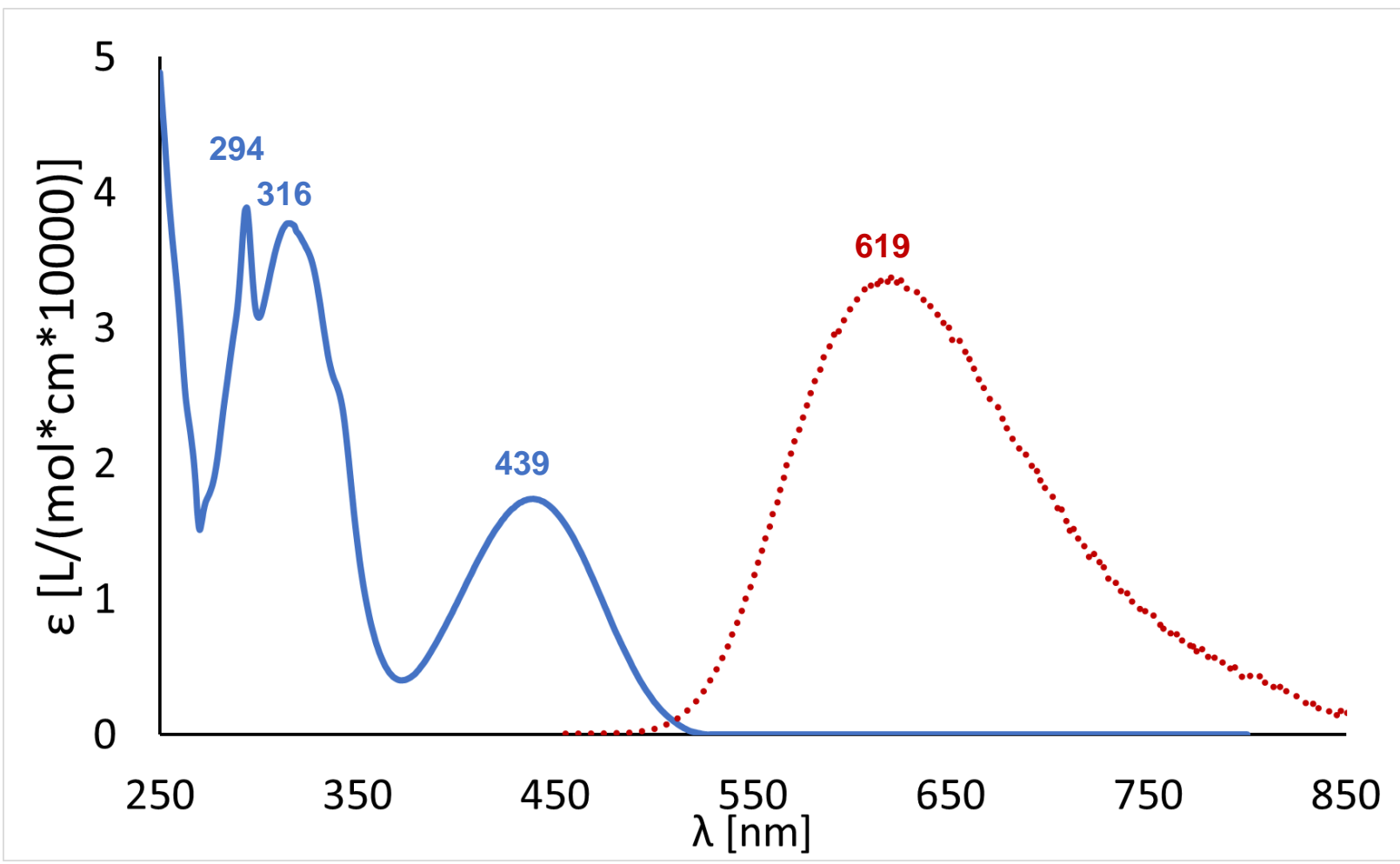

Figure S129. UV-Vis (blue line)- and Fluorescence (red dotted line) spectra of 11a in DCM. 


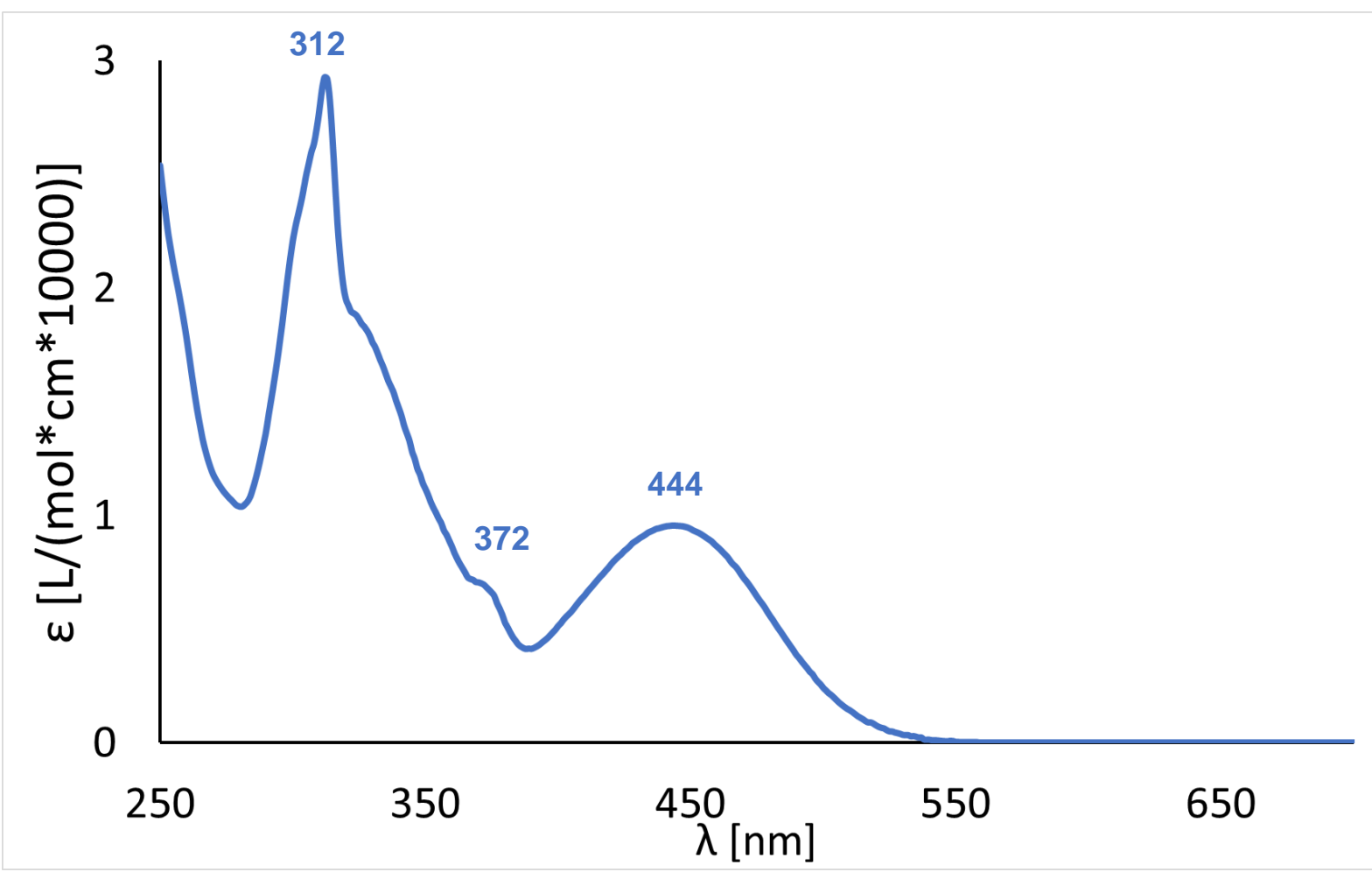

Figure S130. UV-Vis spectra of $\mathbf{1 1 b}$ in DCM.

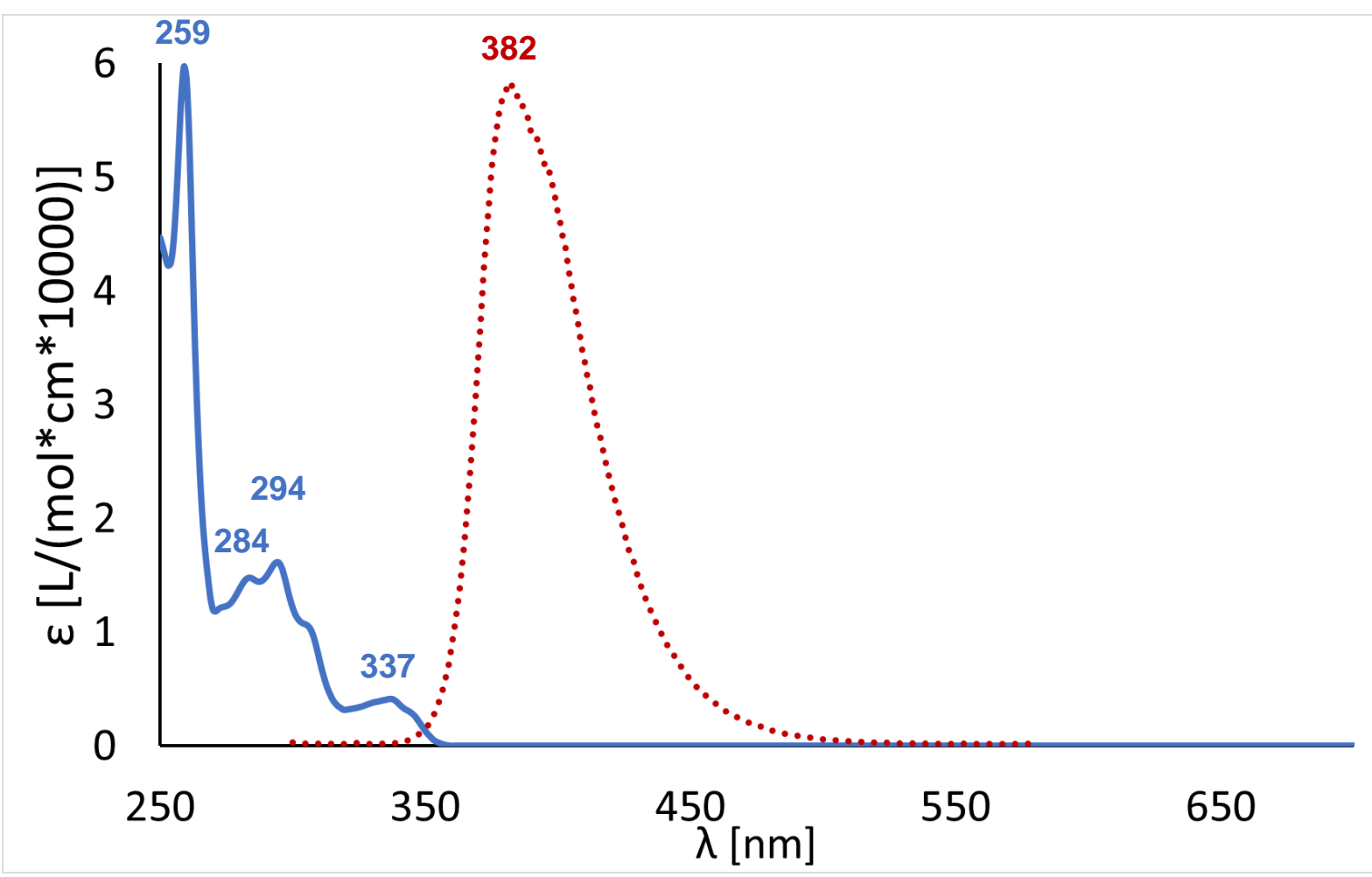

Figure S131. UV-Vis (blue line)- and Fluorescence (red dotted line) spectra of 14(S) in DCM. 


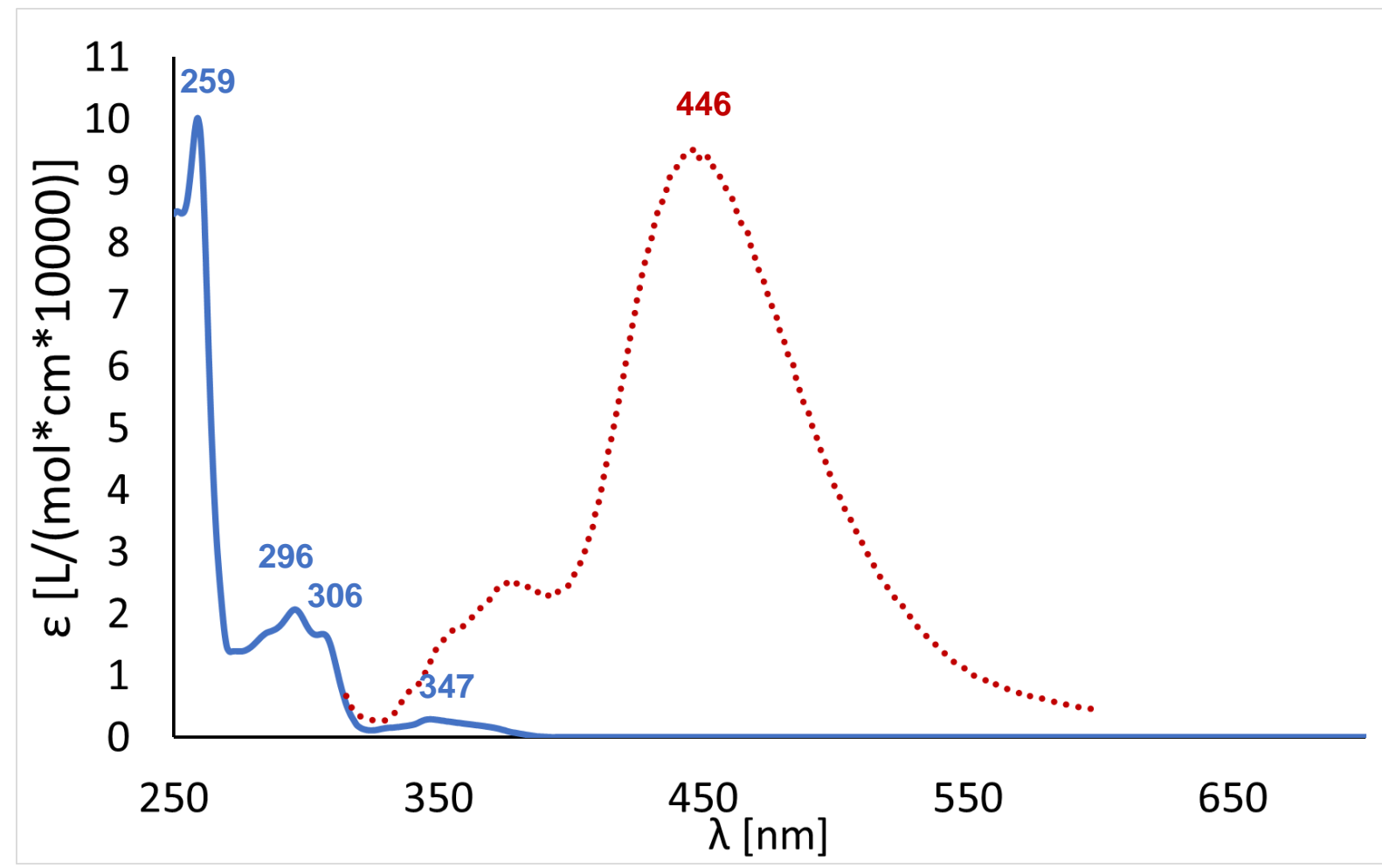

Figure S132. UV-Vis (blue line)- and Fluorescence (red dotted line) spectra of $\mathbf{1 5}(S)$ in DCM.

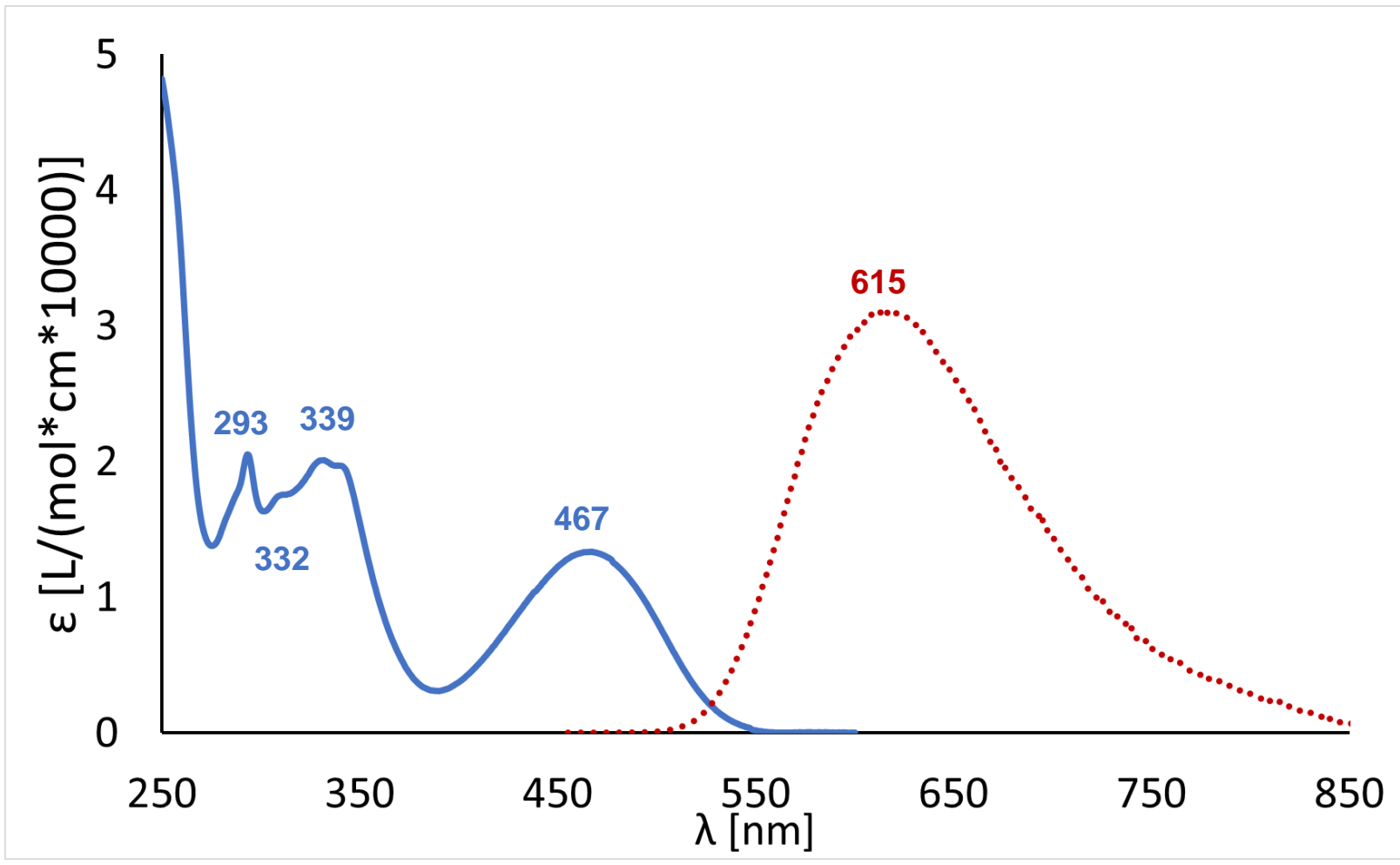

Figure S133. UV-Vis (blue line)- and Fluorescence (red dotted line) spectra of 17(S)a in DCM. 


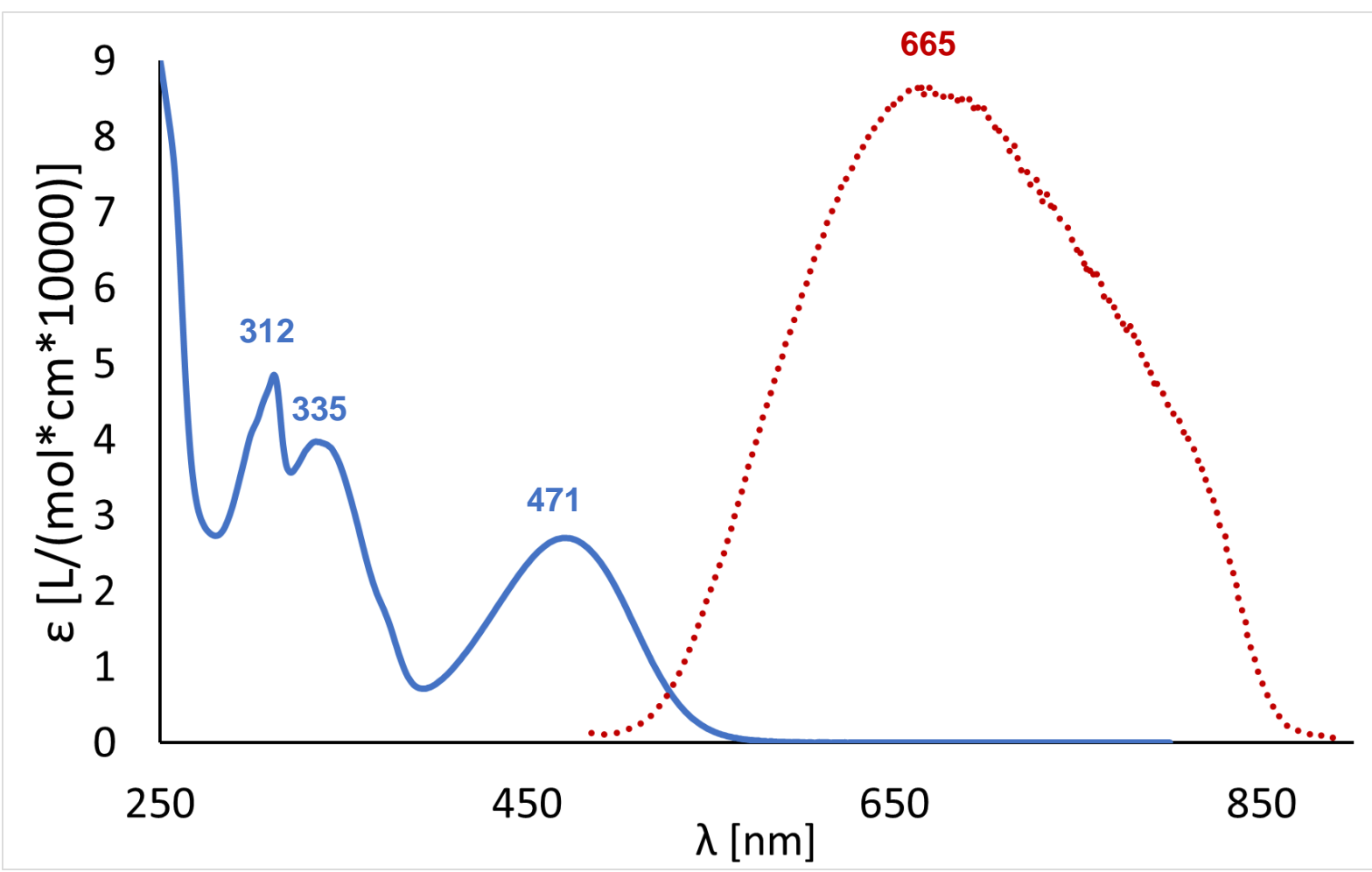

Figure S134. UV-Vis (blue line)- and Fluorescence (red dotted line) spectra of 17(S)b in DCM.

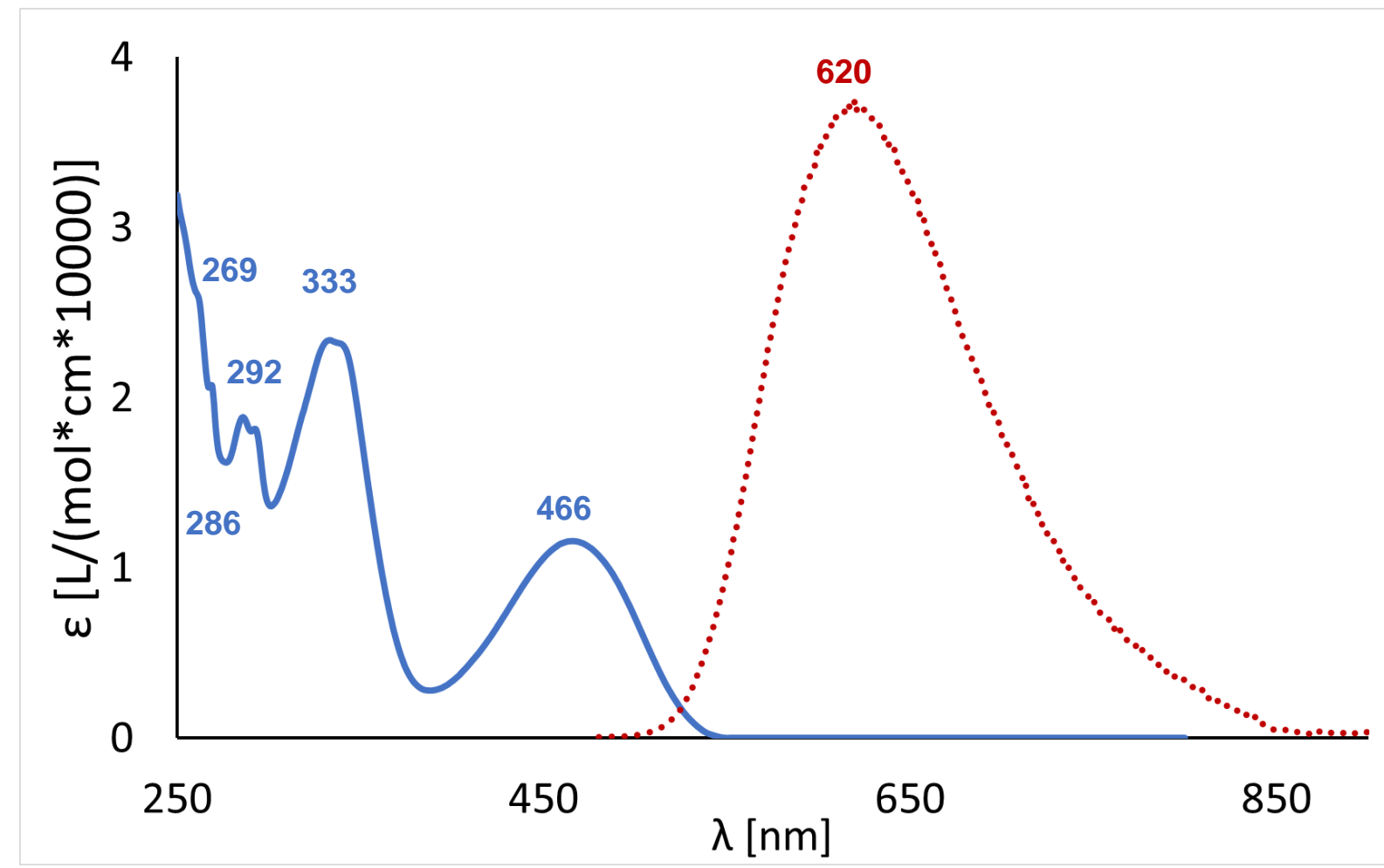

Figure S135. UV-Vis (blue line)- and Fluorescence (red dotted line) spectra of 18(S)a in DCM. 


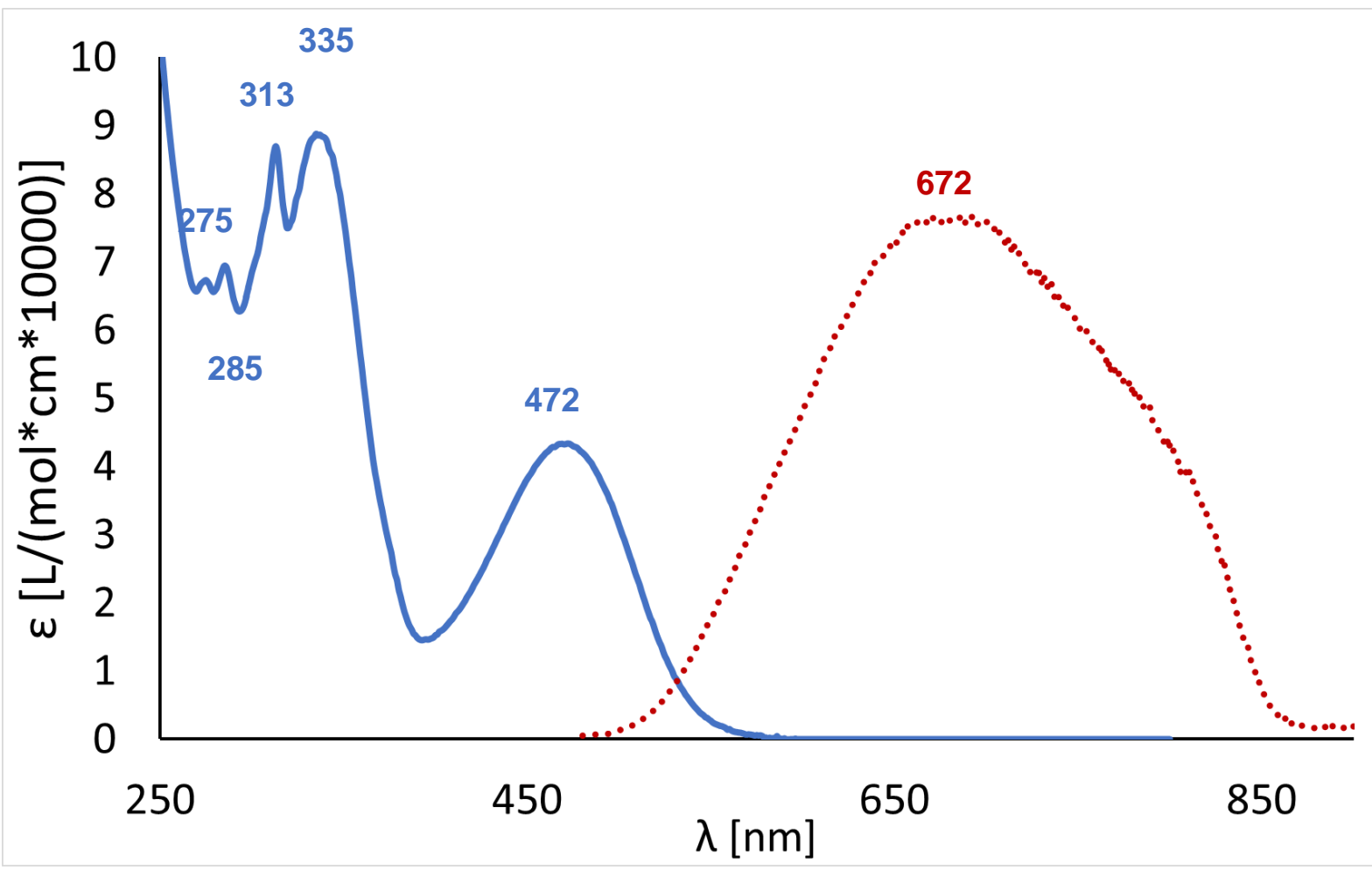

Figure S136. UV-Vis (blue line)- and Fluorescence (red dotted line) spectra of $\mathbf{1 8}(S)$ b in DCM.

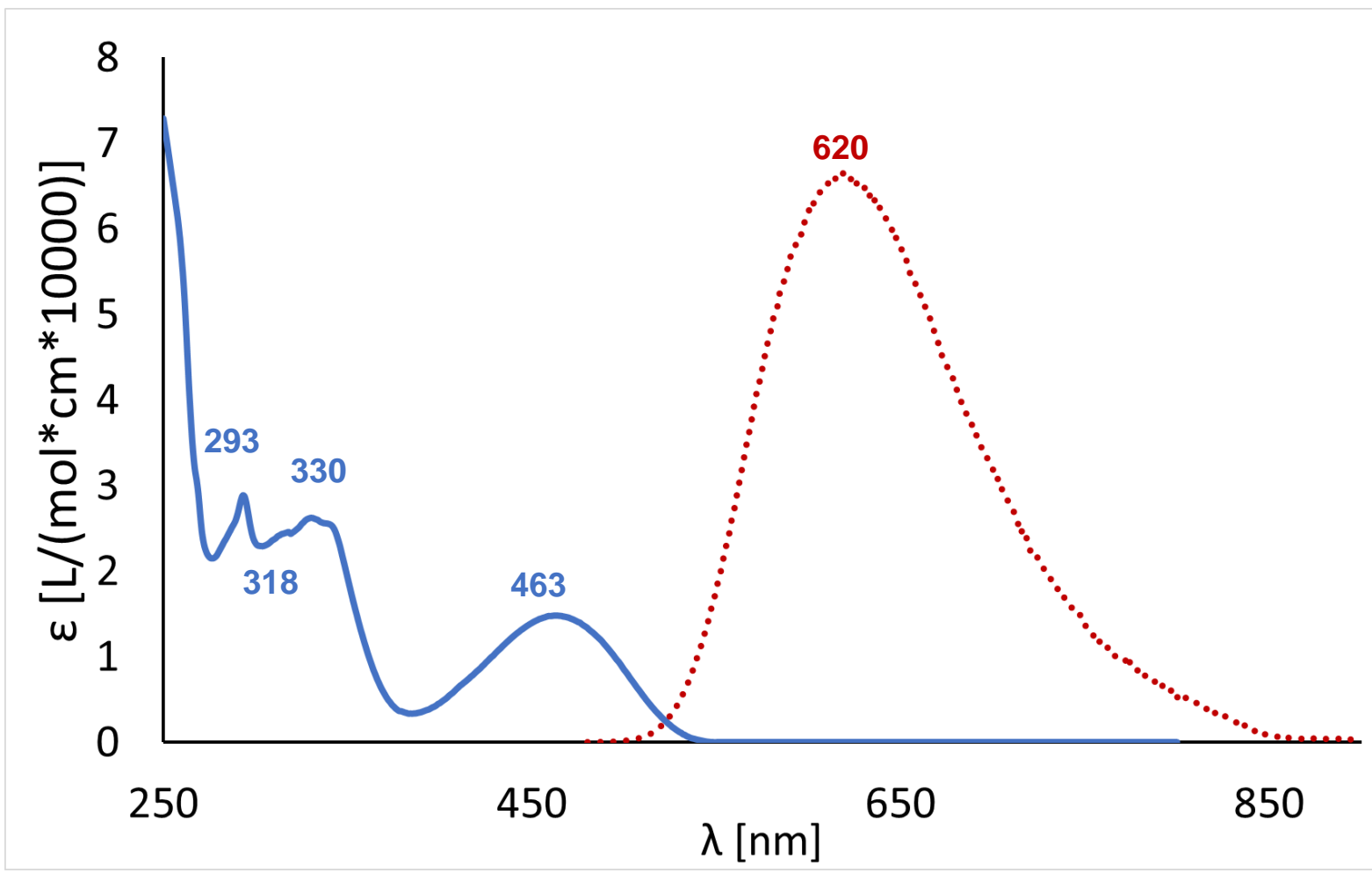

Figure S137. UV-Vis (blue line)- and Fluorescence (red dotted line) spectra of 19(S)a in DCM. 


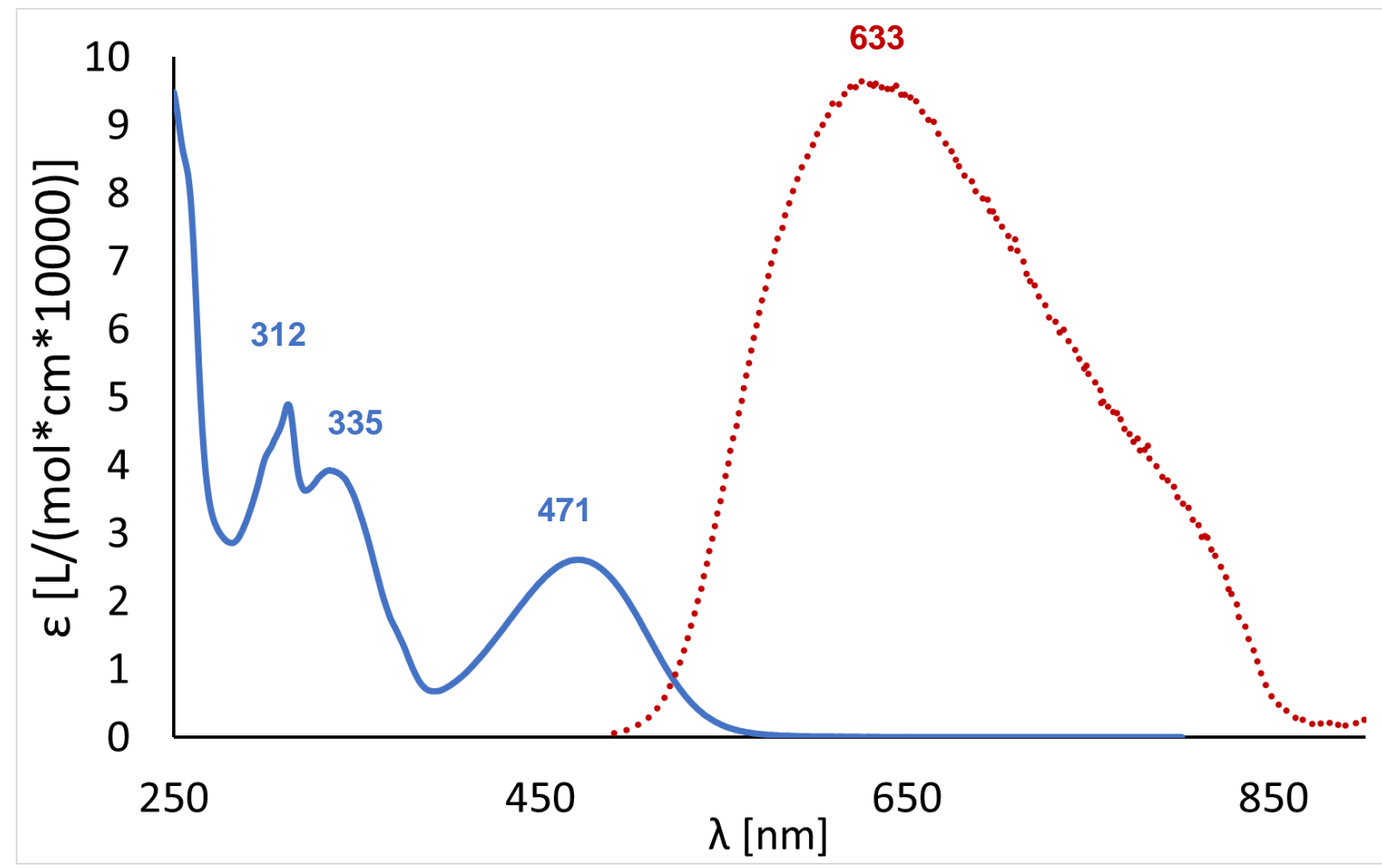

Figure S138. UV-Vis (blue line)- and Fluorescence (red dotted line) spectra of $\mathbf{1 9}(S)$ b in DCM.

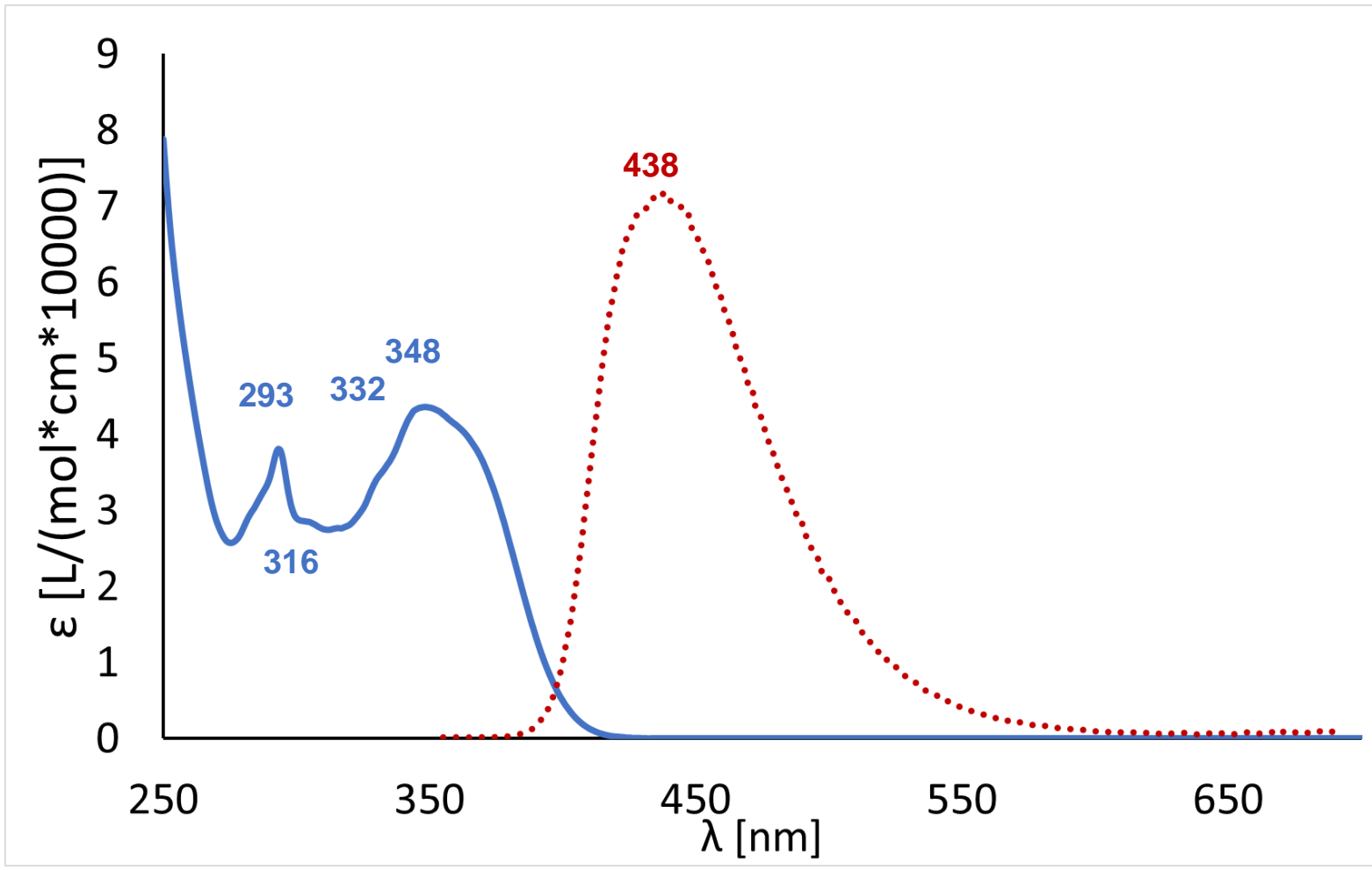

Figure S139. UV-Vis (blue line)- and Fluorescence (red dotted line) spectra of 20(S) in DCM. 


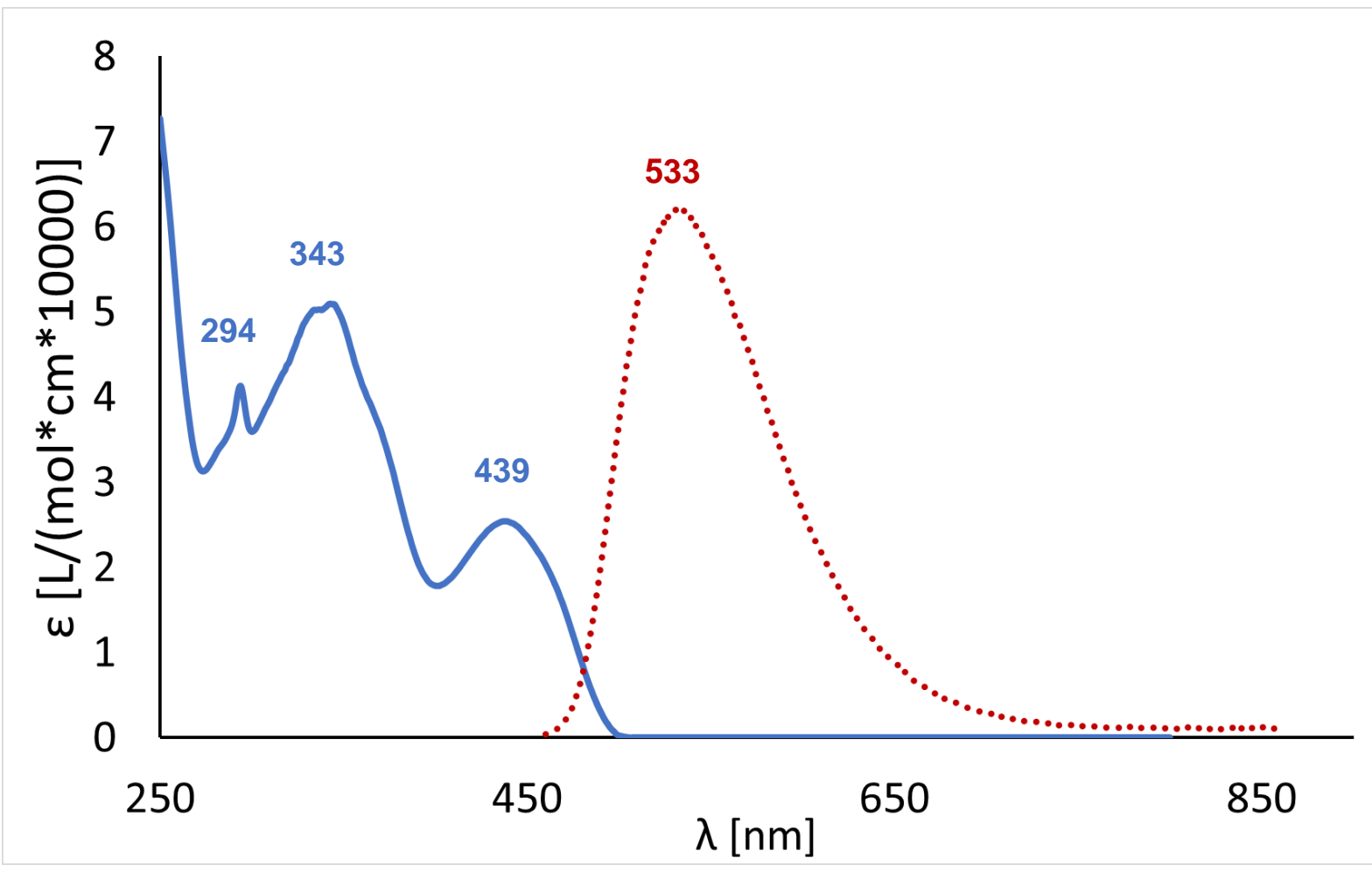

Figure S140. UV-Vis (blue line)- and Fluorescence (red dotted line) spectra of 21(S) in DCM.

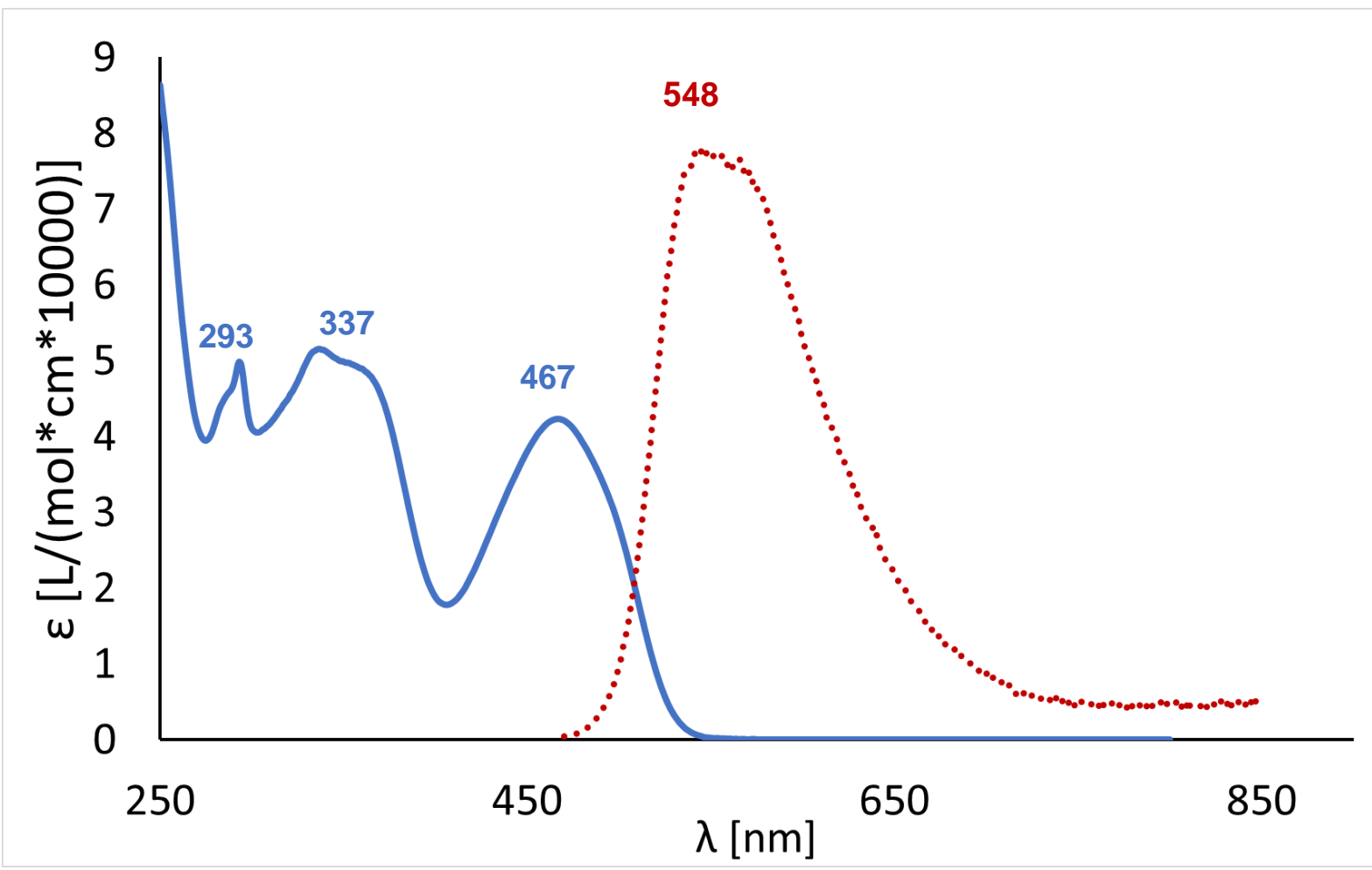

Figure S141. UV-Vis (blue line)- and Fluorescence (red dotted line) spectra of 22(S) in DCM. 


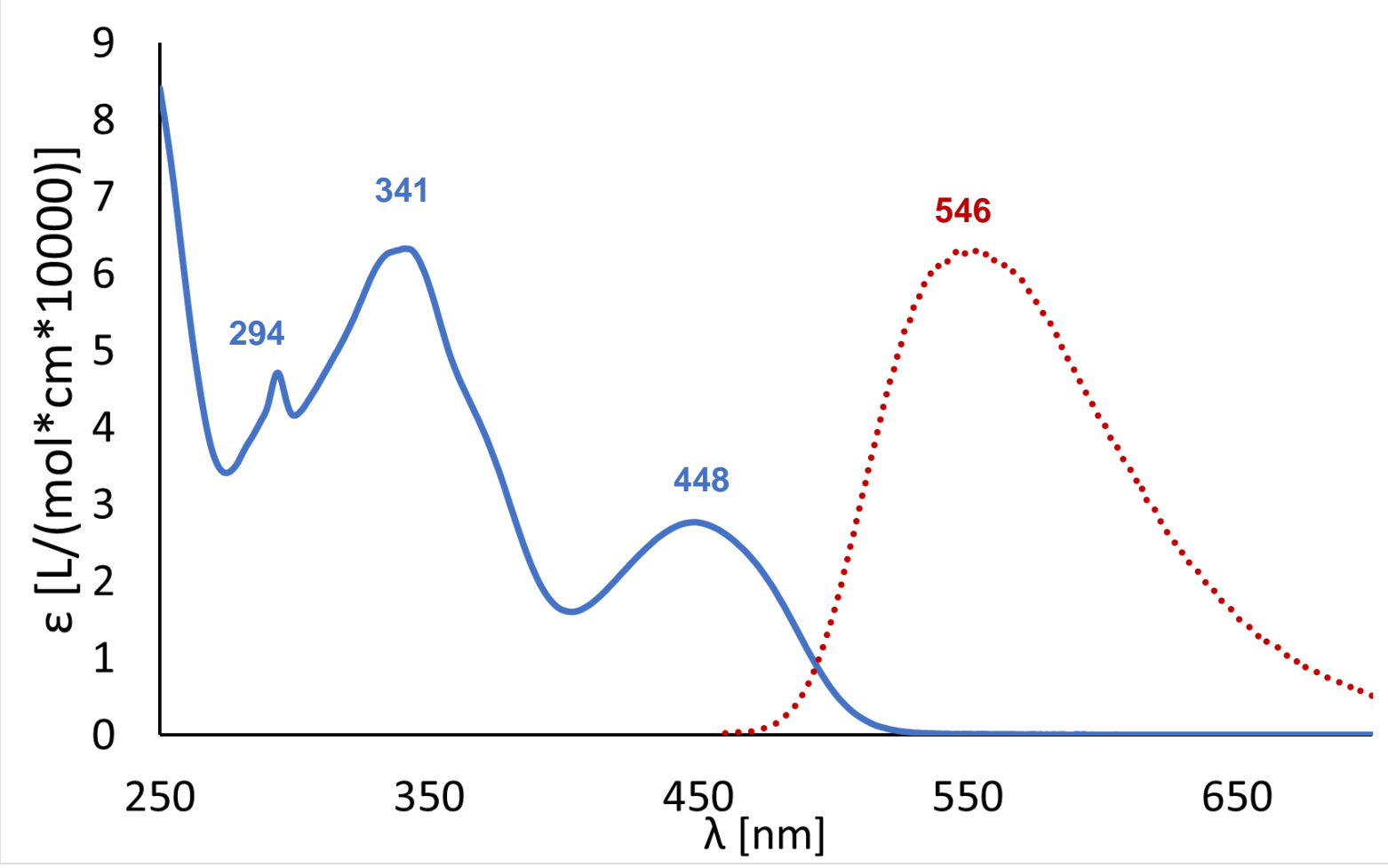

Figure S142. UV-Vis (blue line)- and Fluorescence (red dotted line) spectra of $\mathbf{2 3}(S)$ in DCM. 
Table S1. The UV-Vis absorption maxima with the highest wavelength [nm], fluorescence maxima $[\mathrm{nm}]$, stokes shifts [nm] and fluorescence quantum yields $(\Phi)$, and $\mathrm{R}^{2}$ value of the linear fitting slope. All measurements were carried out in DCM.

\begin{tabular}{|c|c|c|c|c|c|}
\hline Molecule & $\begin{array}{c}\mathrm{UV} \text {-Vis maximum } \\
{[\mathrm{nm}]}\end{array}$ & $\begin{array}{c}\text { Emission maximum } \\
{[\mathrm{nm}]}\end{array}$ & $\begin{array}{c}\text { Stokes shift } \\
{[\mathrm{nm}]}\end{array}$ & $\Phi[\%]$ & $\begin{array}{l}\mathbf{R}^{2} \text { for } \\
\quad \Phi\end{array}$ \\
\hline $2 b$ & 370 & 387 & 17 & 3 & 0.997 \\
\hline $4 a$ & 340 & 367 & 27 & 23 & 0.999 \\
\hline $4 b$ & 369 & 390 & 21 & 20 & 0.999 \\
\hline 6 & 327 & 414 & 87 & 37 & 0.999 \\
\hline 7 & 324 & 410 & 86 & 31 & 0.999 \\
\hline 9 & 414 & 504 & 90 & 27 & 0.997 \\
\hline $11 a$ & 441 & 619 & 178 & 39 & 0.999 \\
\hline $14(S)$ & 337 & 382 & 45 & 24 & 0.999 \\
\hline $15(S)$ & 347 & 446 & 99 & 1 & 0.999 \\
\hline $17(S) \mathbf{a}$ & 465 & 615 & 150 & 54 & 0.999 \\
\hline $17(S) b$ & 471 & 665 & 194 & 1 & 0.997 \\
\hline $18(S) \mathrm{a}$ & 466 & 620 & 154 & 50 & 0.996 \\
\hline $18(S) b$ & 472 & 672 & 200 & 2 & 0.990 \\
\hline $19(S) \mathbf{a}$ & 464 & 620 & 156 & 47 & 0.995 \\
\hline $19(S) b$ & 471 & 633 & 162 & 1 & 0.999 \\
\hline $20(S)$ & 348 & 438 & 90 & 38 & 0.997 \\
\hline $21(S)$ & 439 & 533 & 94 & 6 & 0.999 \\
\hline $22(S)$ & 467 & 548 & 81 & 5 & 0.998 \\
\hline $23(S)$ & 448 & 546 & 98 & 5 & 0.997 \\
\hline
\end{tabular}




\section{CW-EPR spectra}

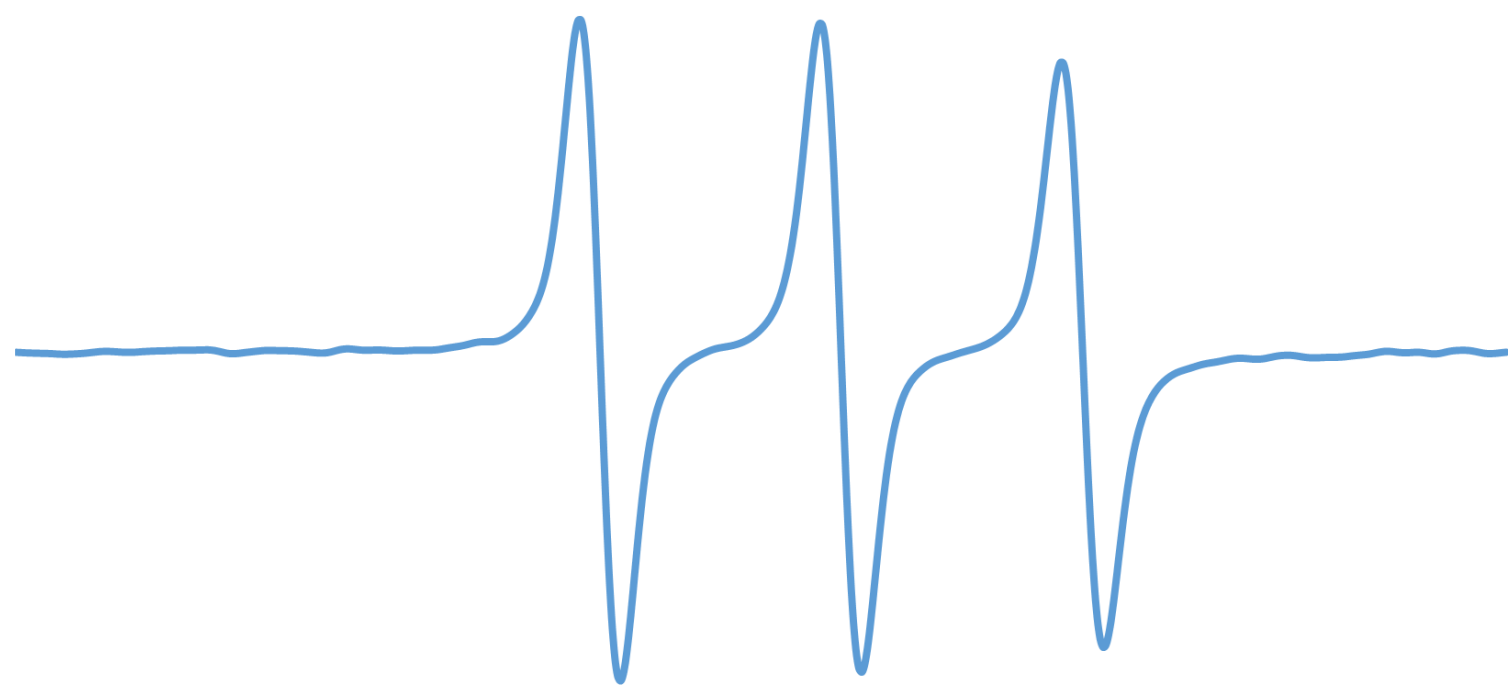

3450

3475

3500

3525

Magnetic Field [G]

Figure S143. CW-EPR spectra of compound 19(S)a at X-band $(9.8 \mathrm{GHz})$ in DCM obtained at room temperature with a $2.0 \mathrm{G}$ modulation amplitude.

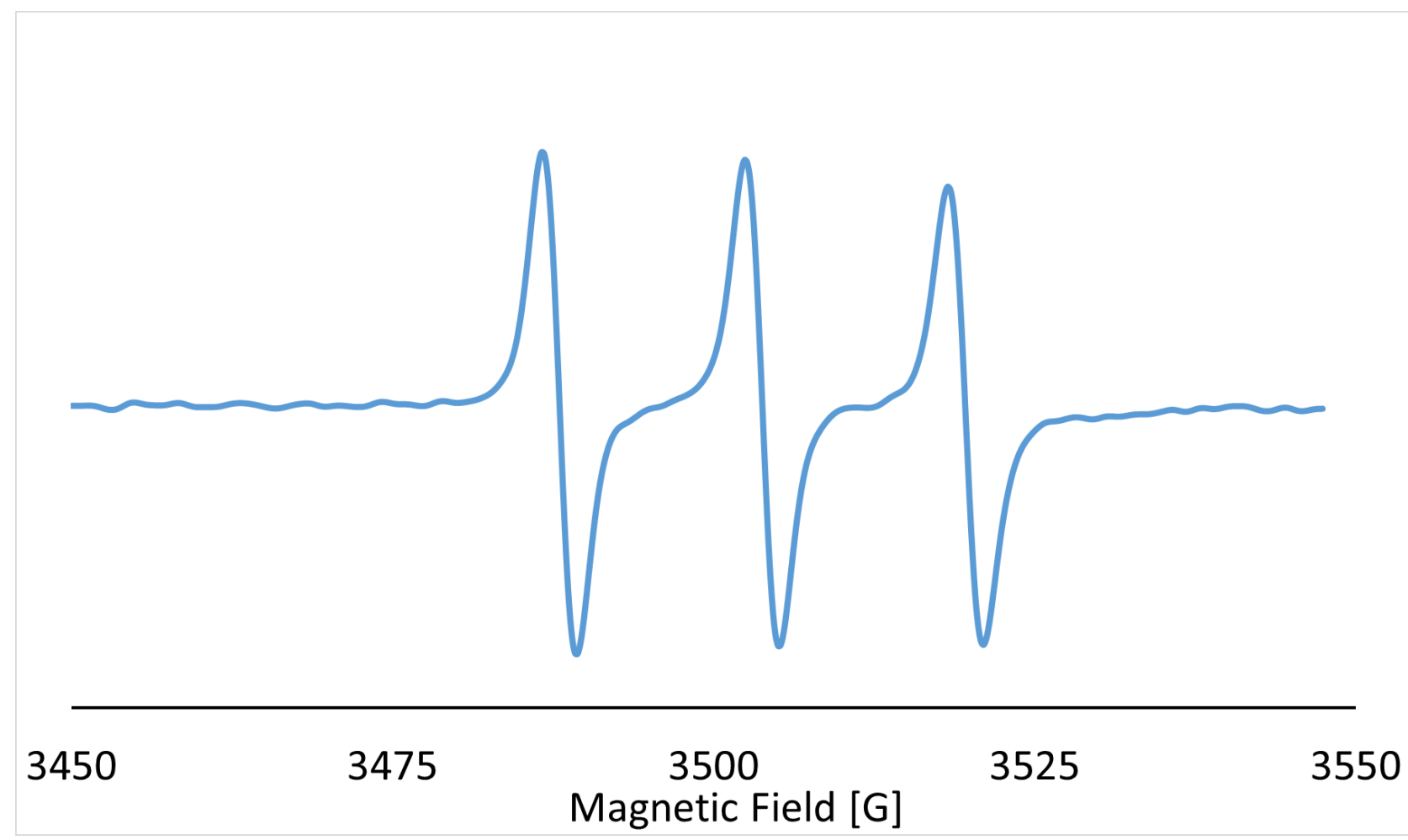

Figure S144. CW-EPR spectra of compound $19(S)$ b at X-band $(9.8 \mathrm{GHz})$ in DCM obtained at room temperature with a $2.0 \mathrm{G}$ modulation amplitude. 


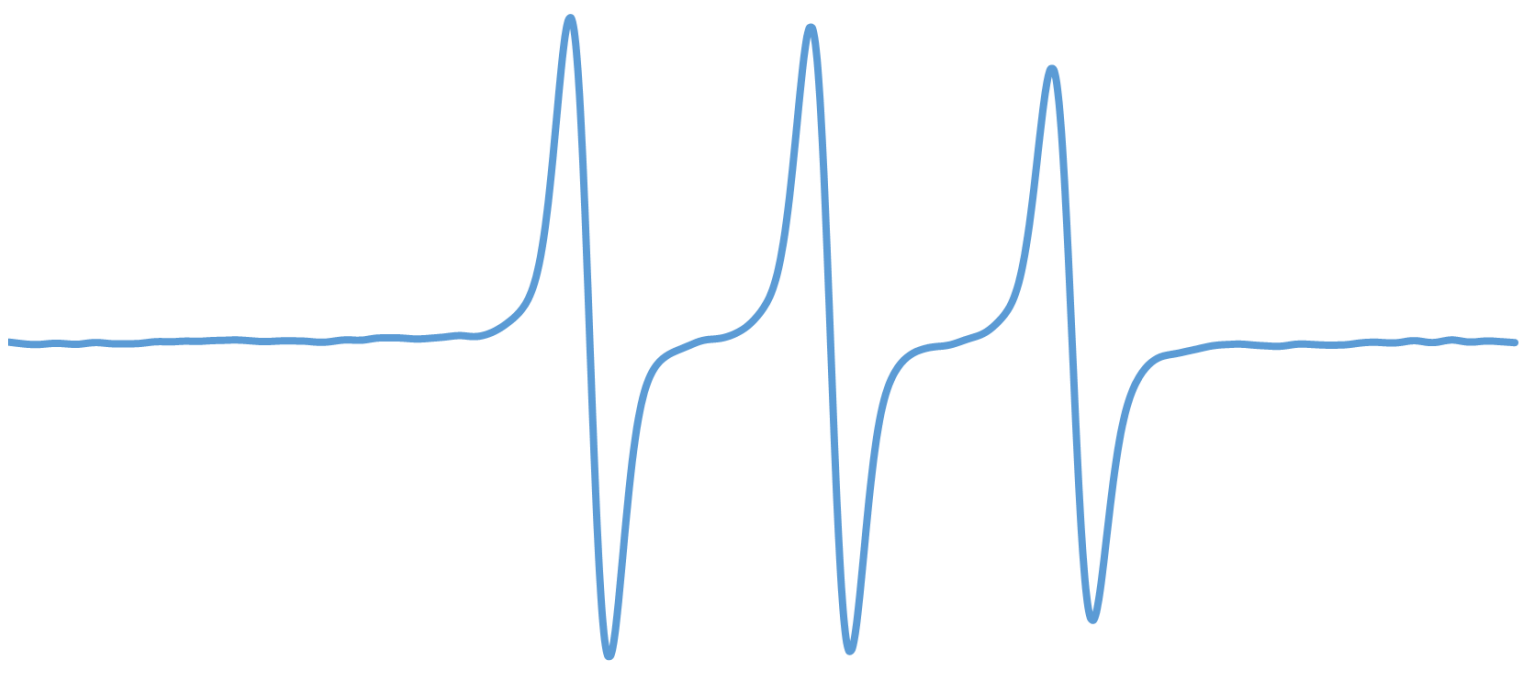

3450

3475

3500

3525

3550

Magnetic Field [G]

Figure S145. CW-EPR spectra of compound $\mathbf{2 3}(S)$ at X-band $(9.8 \mathrm{GHz})$ in DCM obtained at room temperature with a $2.0 \mathrm{G}$ modulation amplitude. 


\section{SQUID measurements}

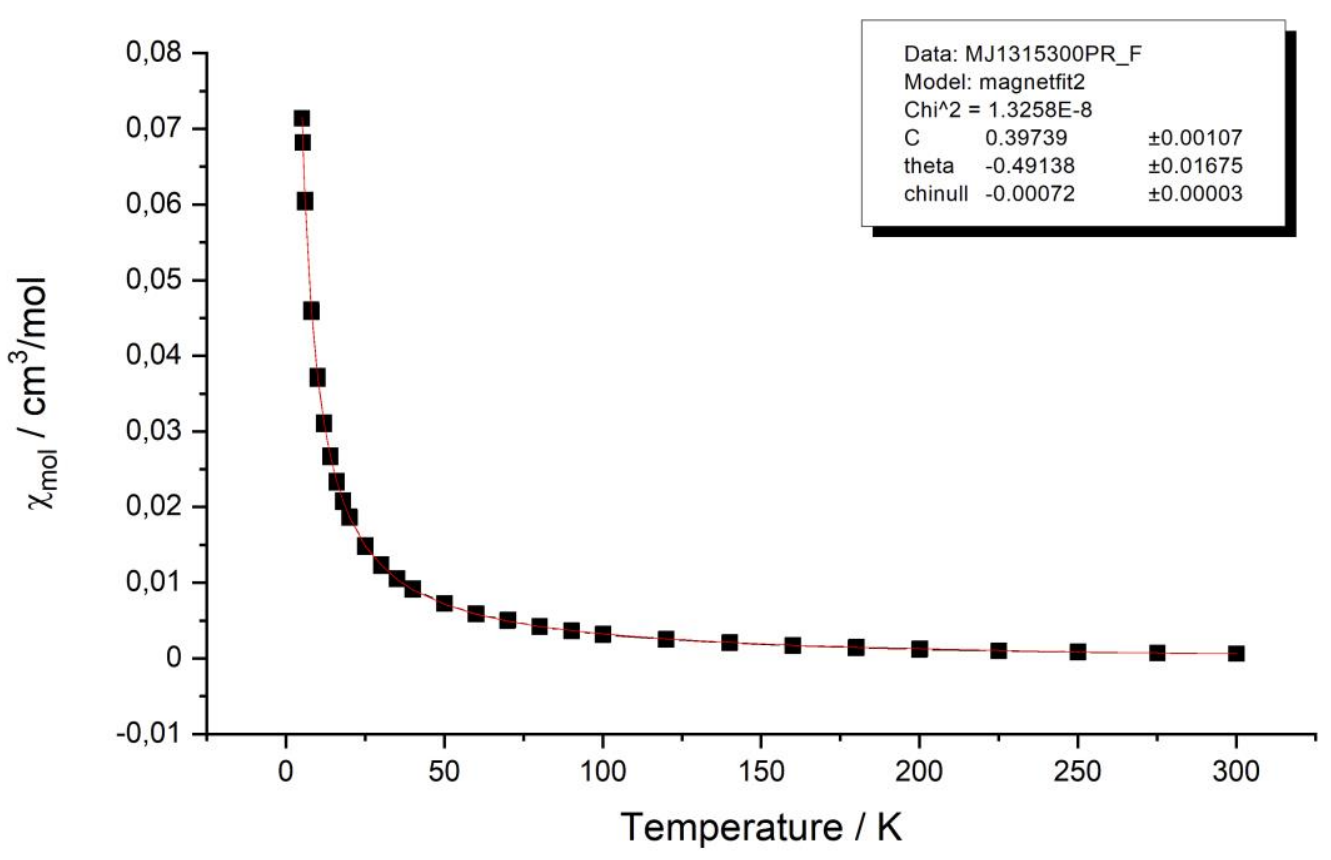

Figure S146. SQUID measurement of $\mathbf{1 9}(S)$ a.

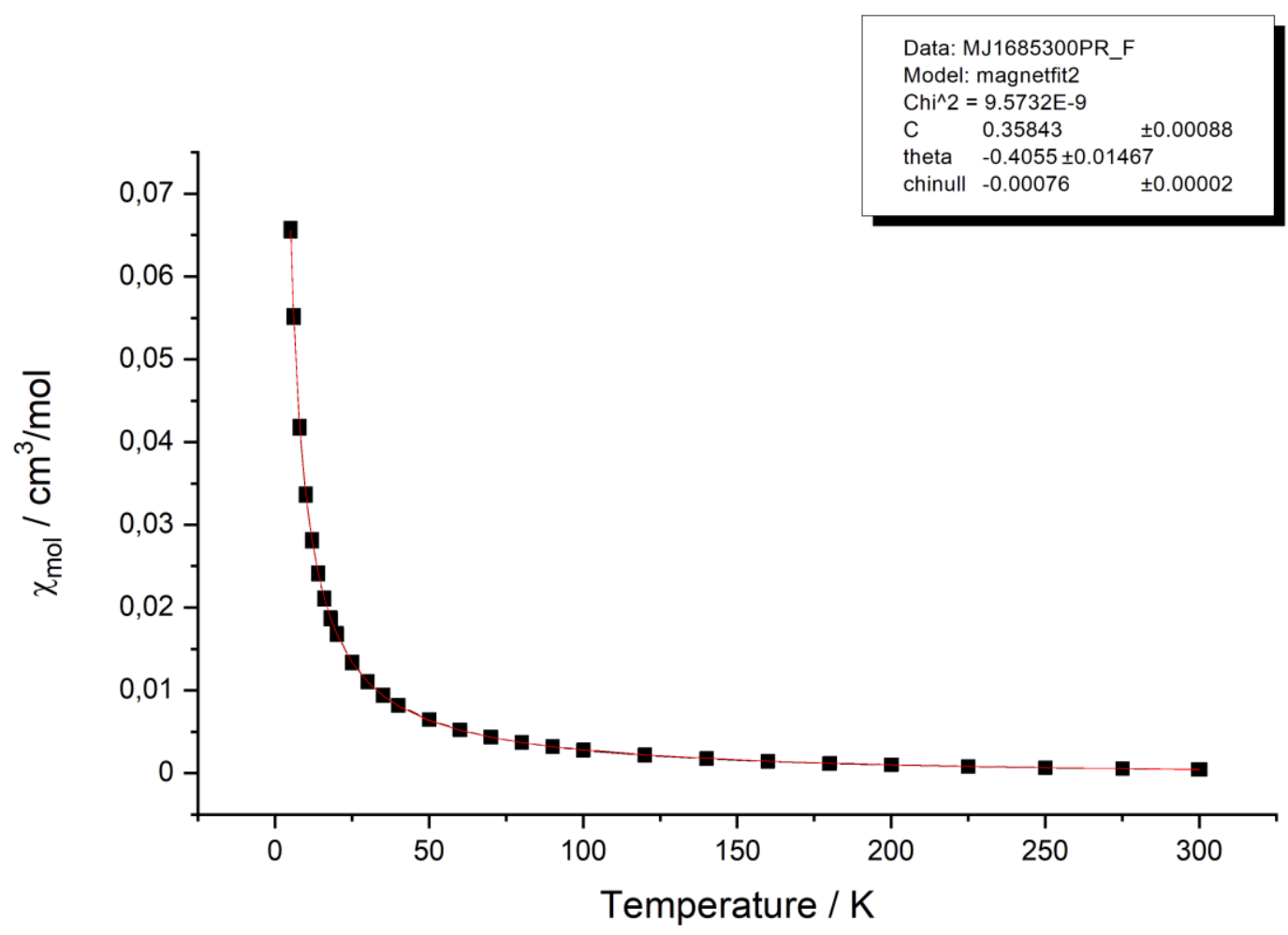

Figure S147. SQUID measurement of $\mathbf{2 3}(S)$. 


\section{ESI/APCI-High Resolution Mass Spectrum}

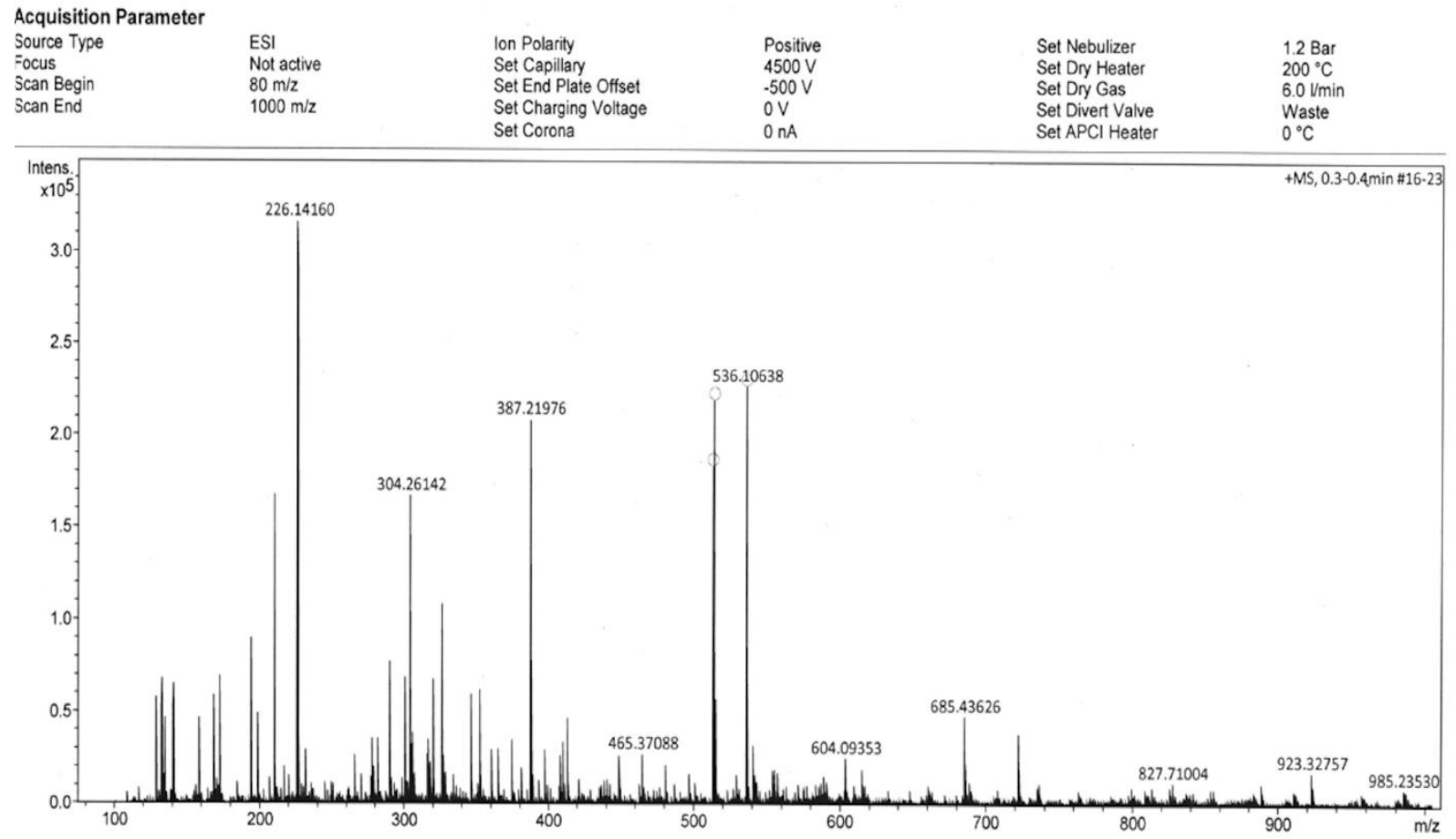

Figure S148. HRMS (ESI) of $2 \mathbf{b}$.

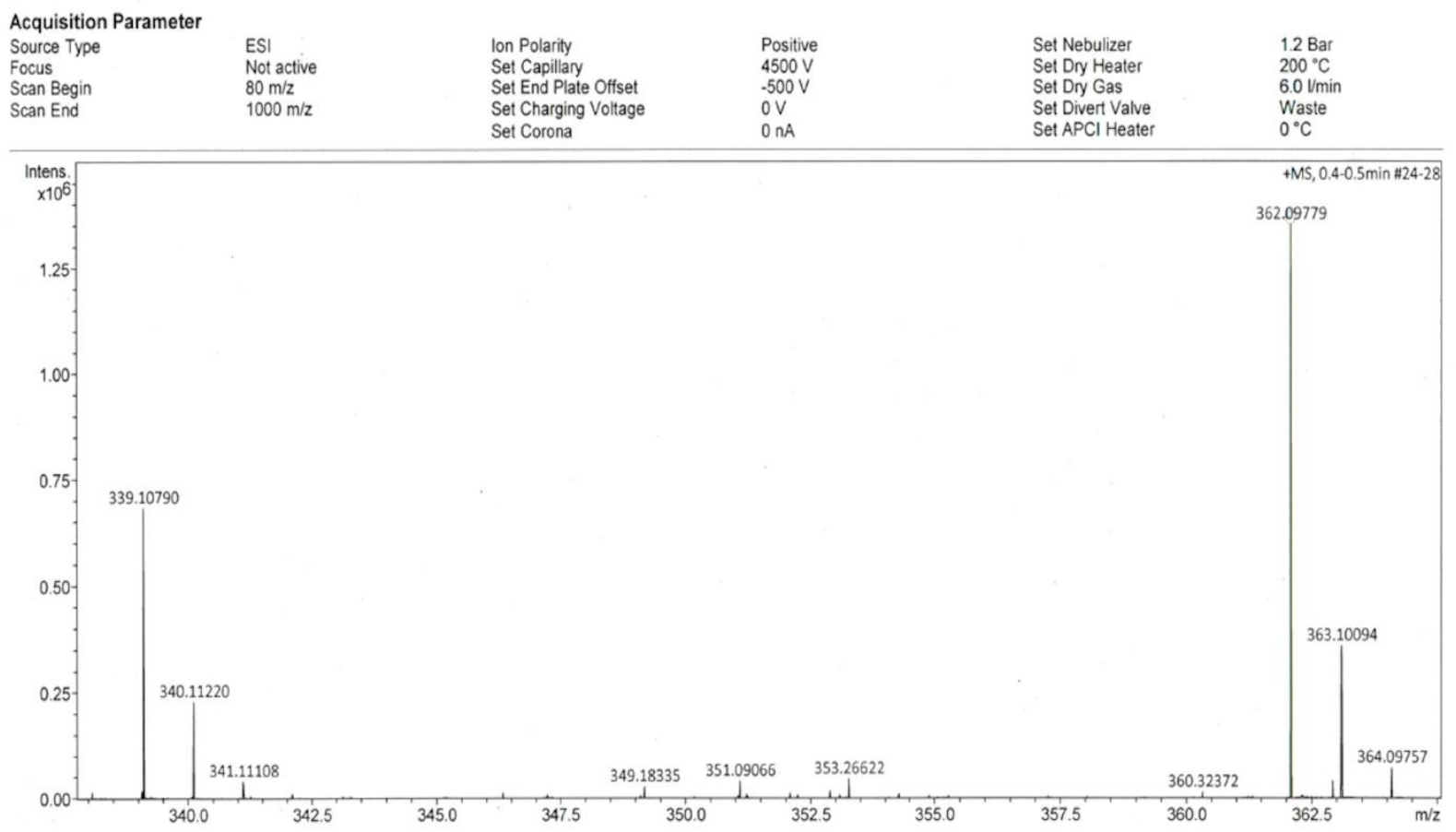

Figure S149. HRMS (ESI) of $4 a$. 


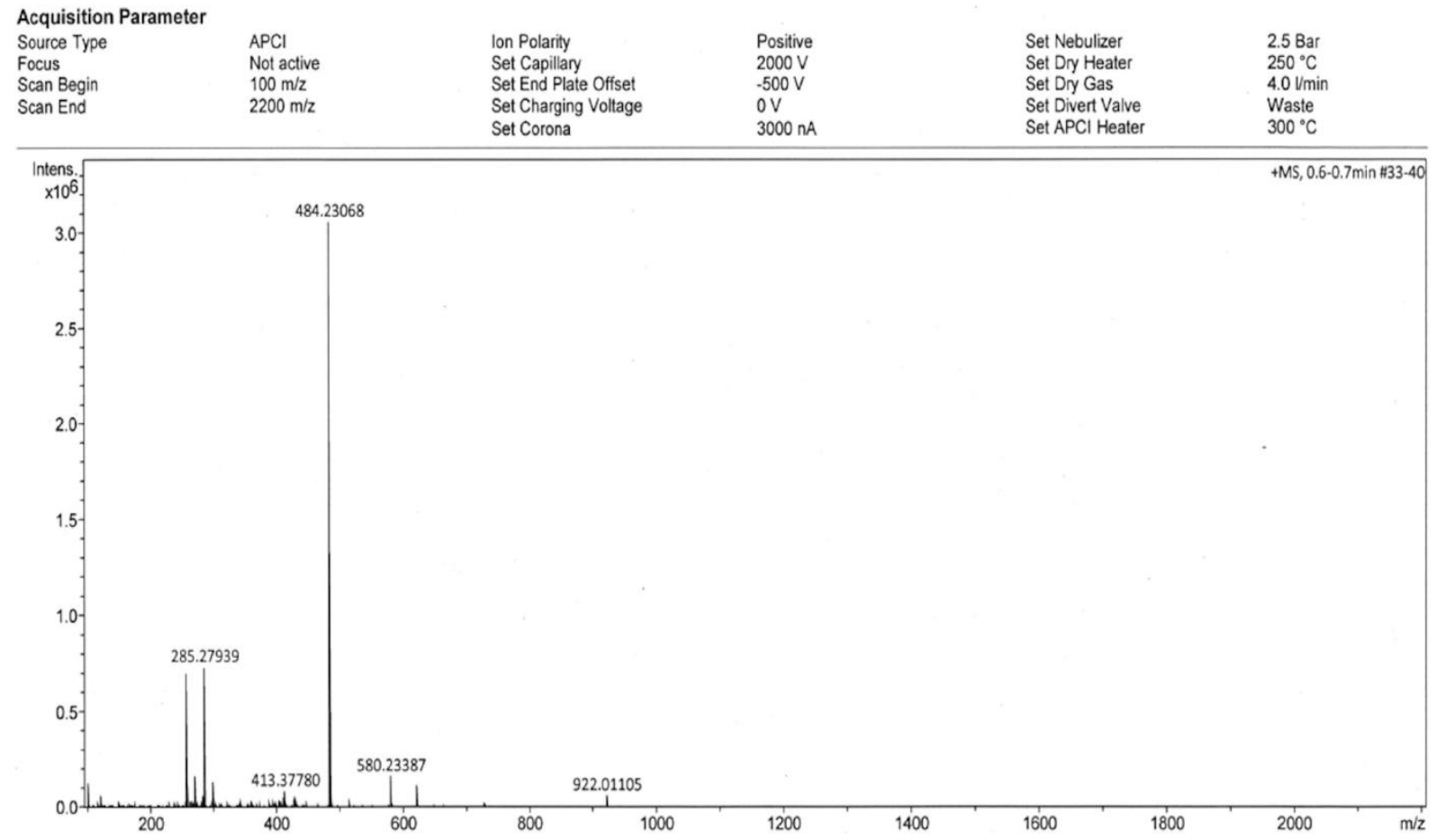

Figure S150. HRMS (APCI) of $\mathbf{4 b}$.

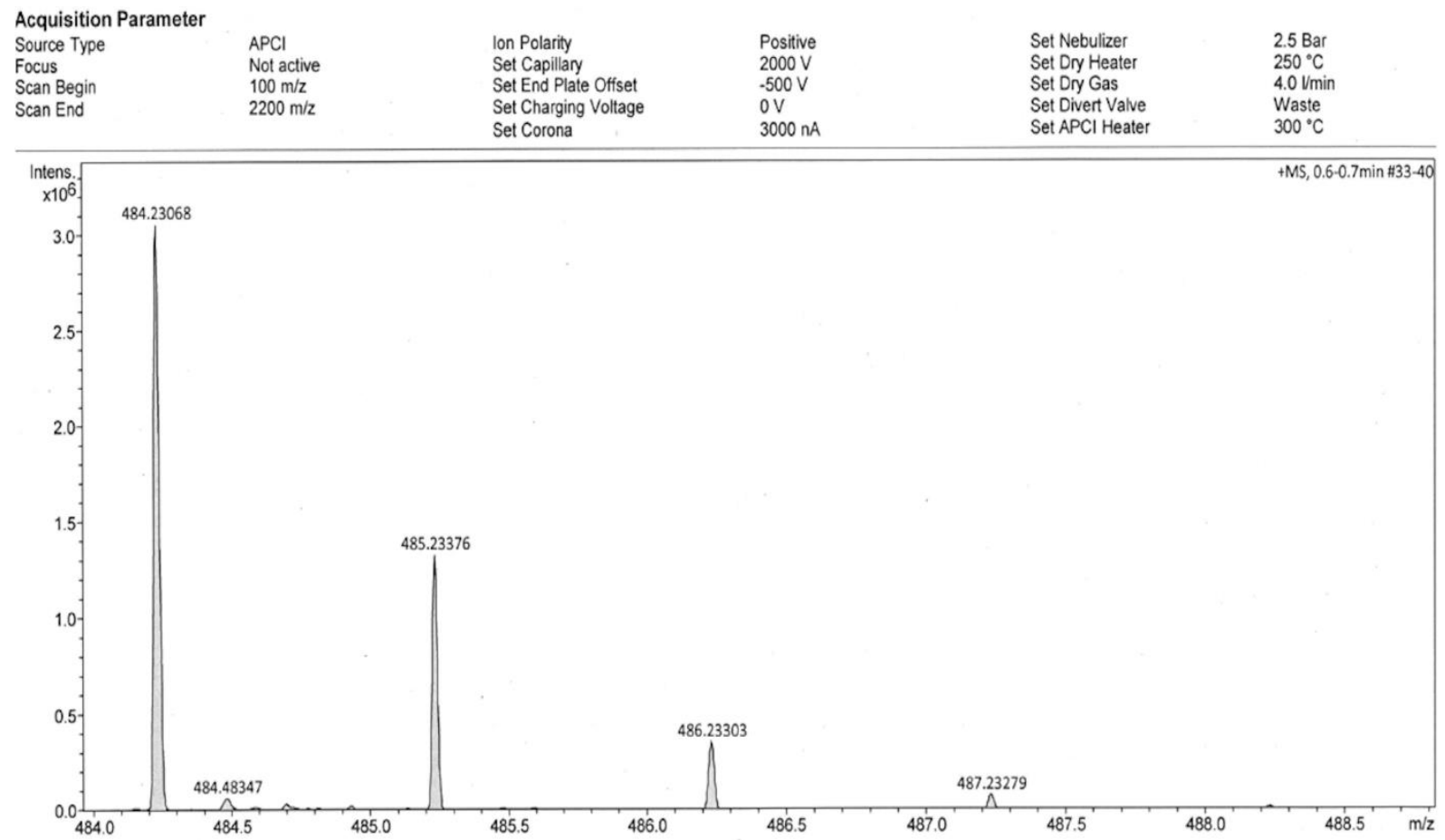

Figure S151. HRMS (APCI) of $\mathbf{4 b .}$ 


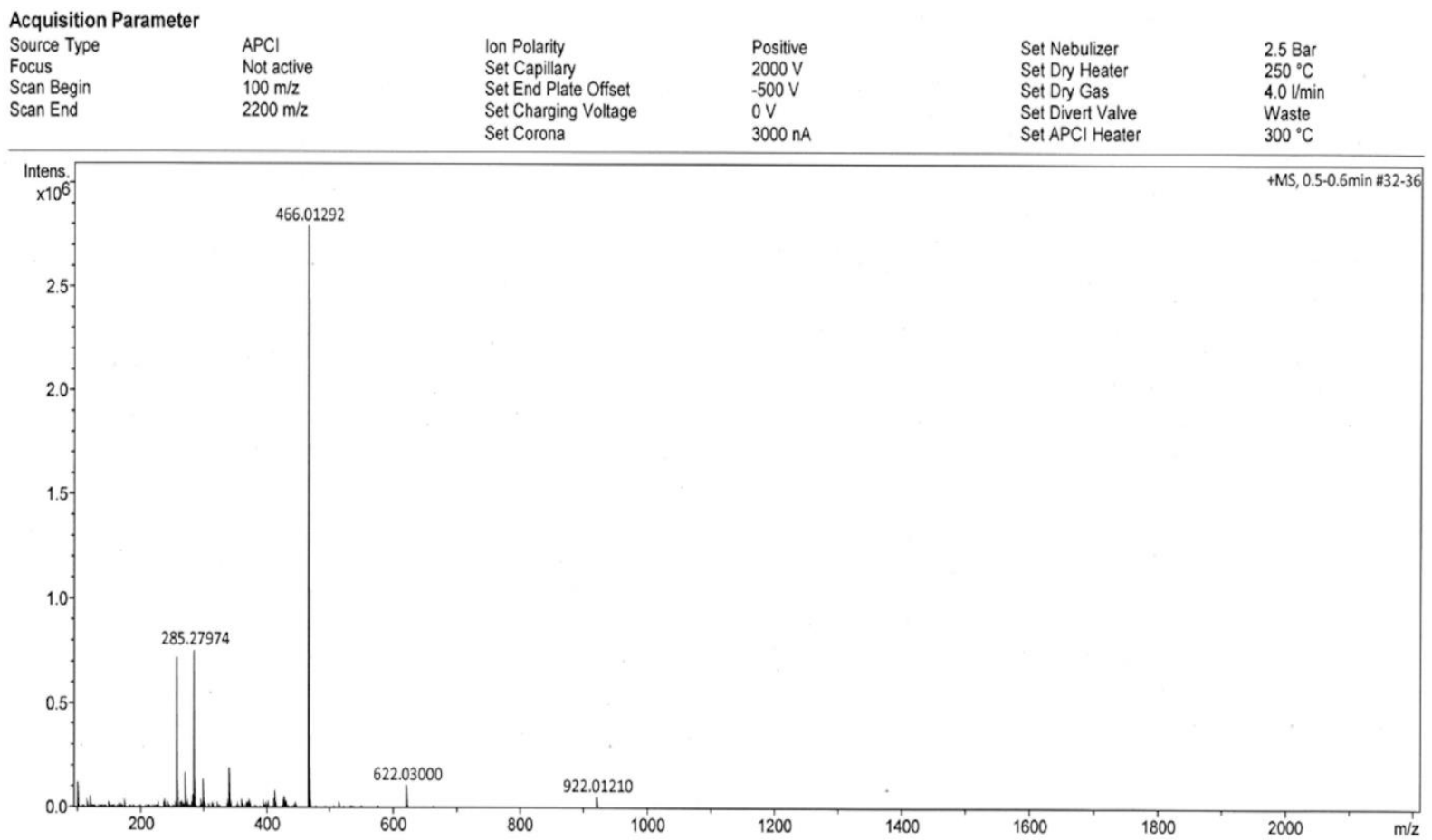

Figure S152. HRMS (APCI) of $\mathbf{5 a}$.

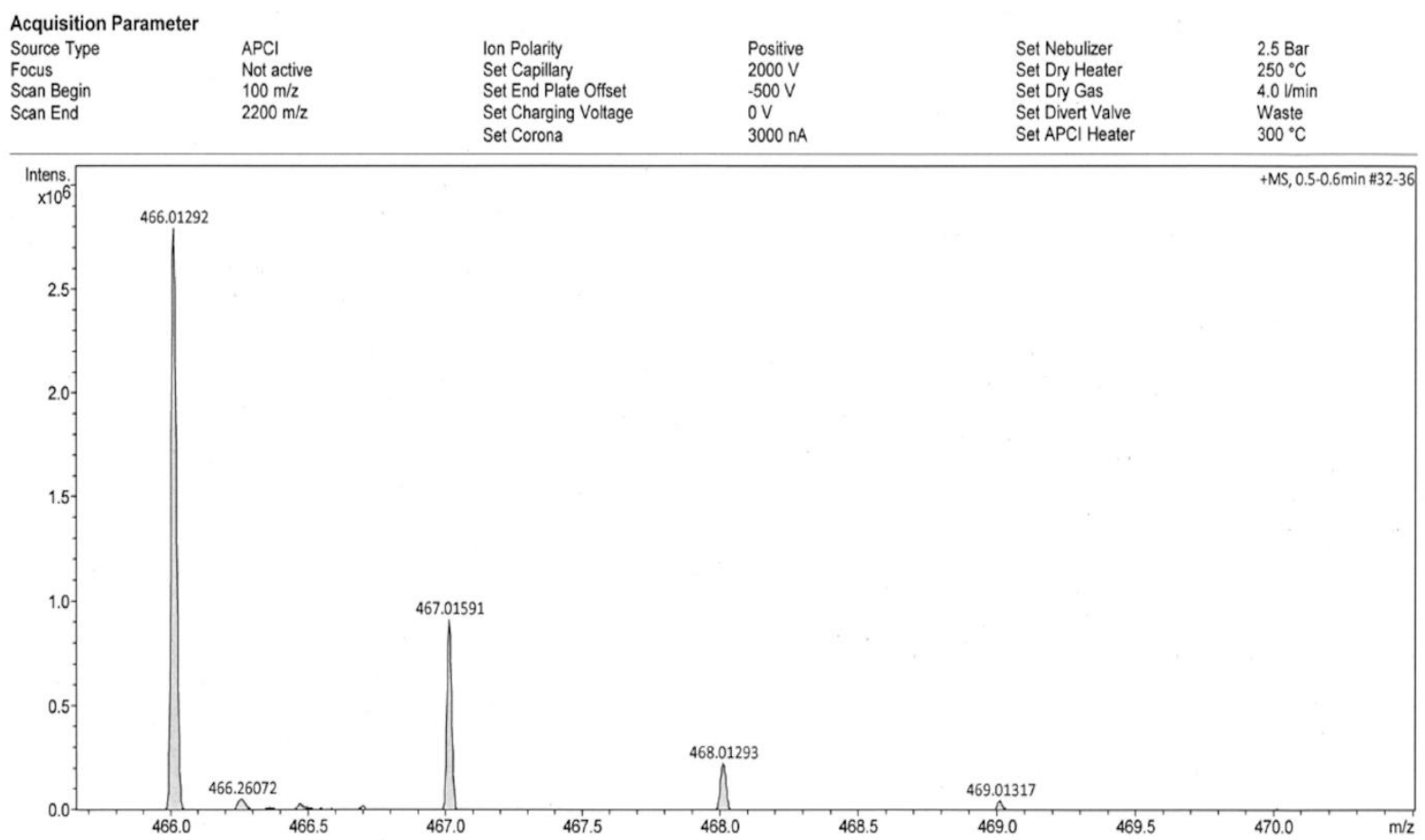

Figure S153. HRMS (APCI) of $\mathbf{5 a}$. 


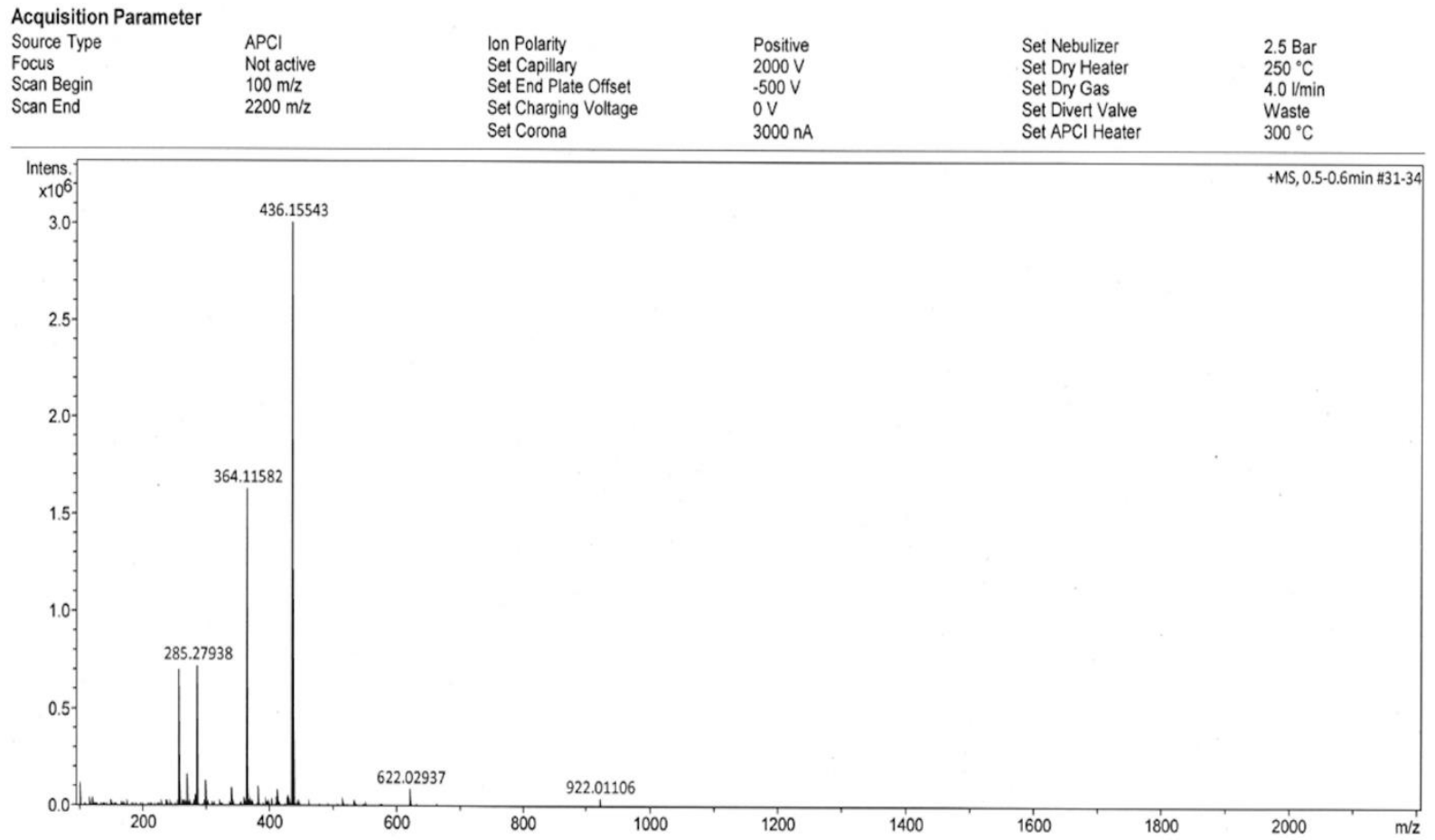

Figure S154. HRMS (APCI) of 6.

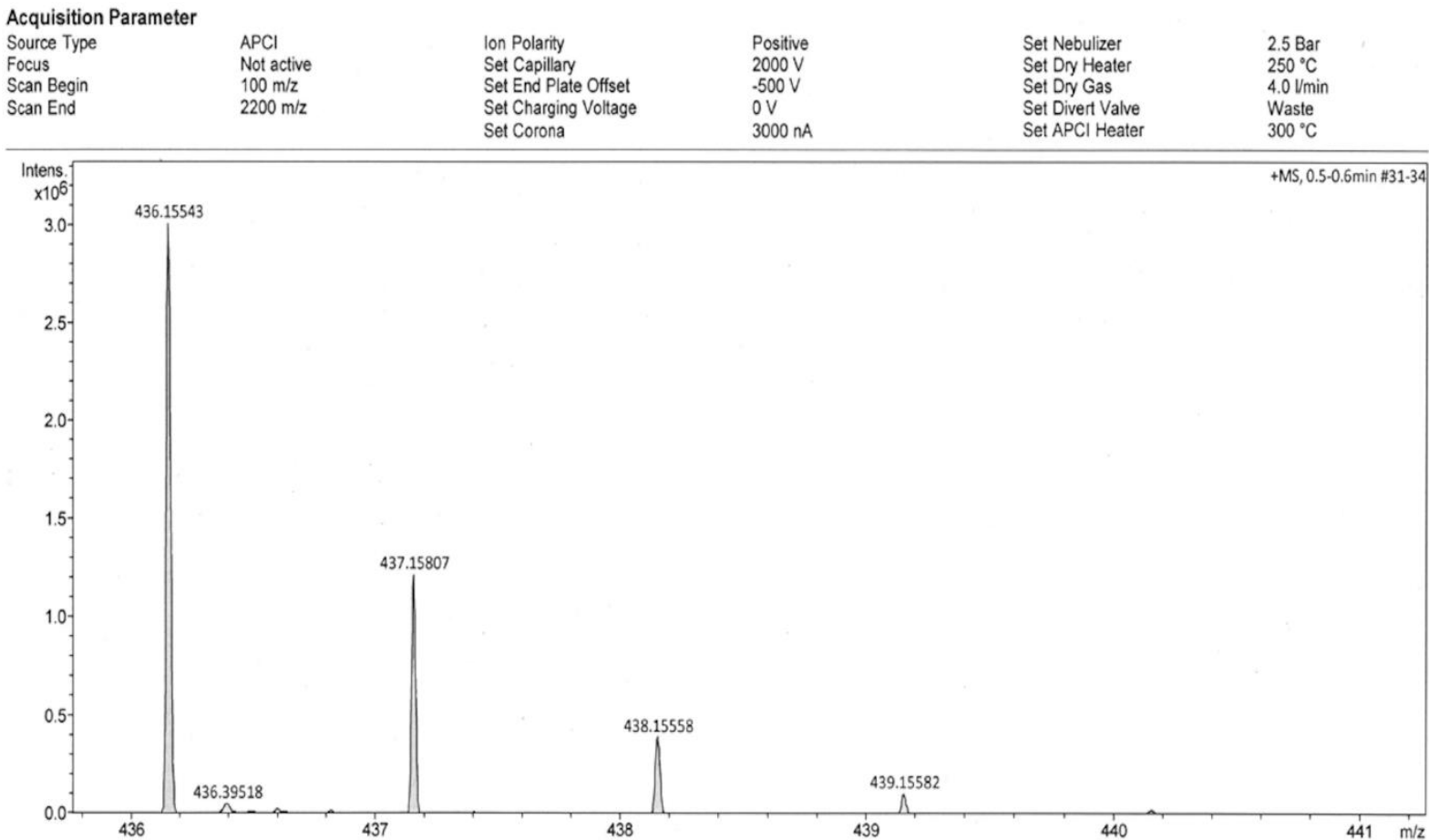

Figure S155. HRMS (APCI) of 6. 


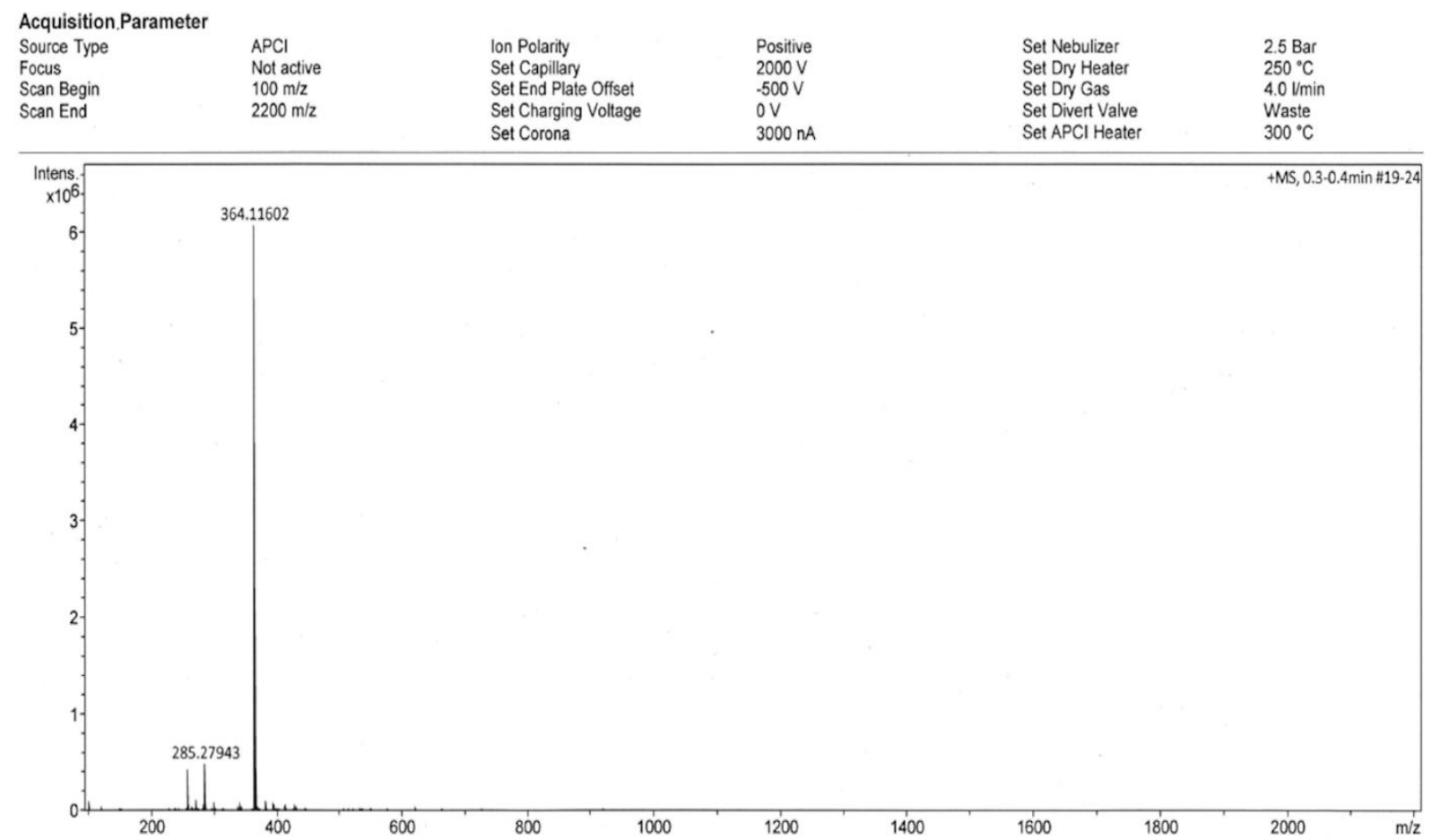

Figure S156. HRMS (APCI) of 7.

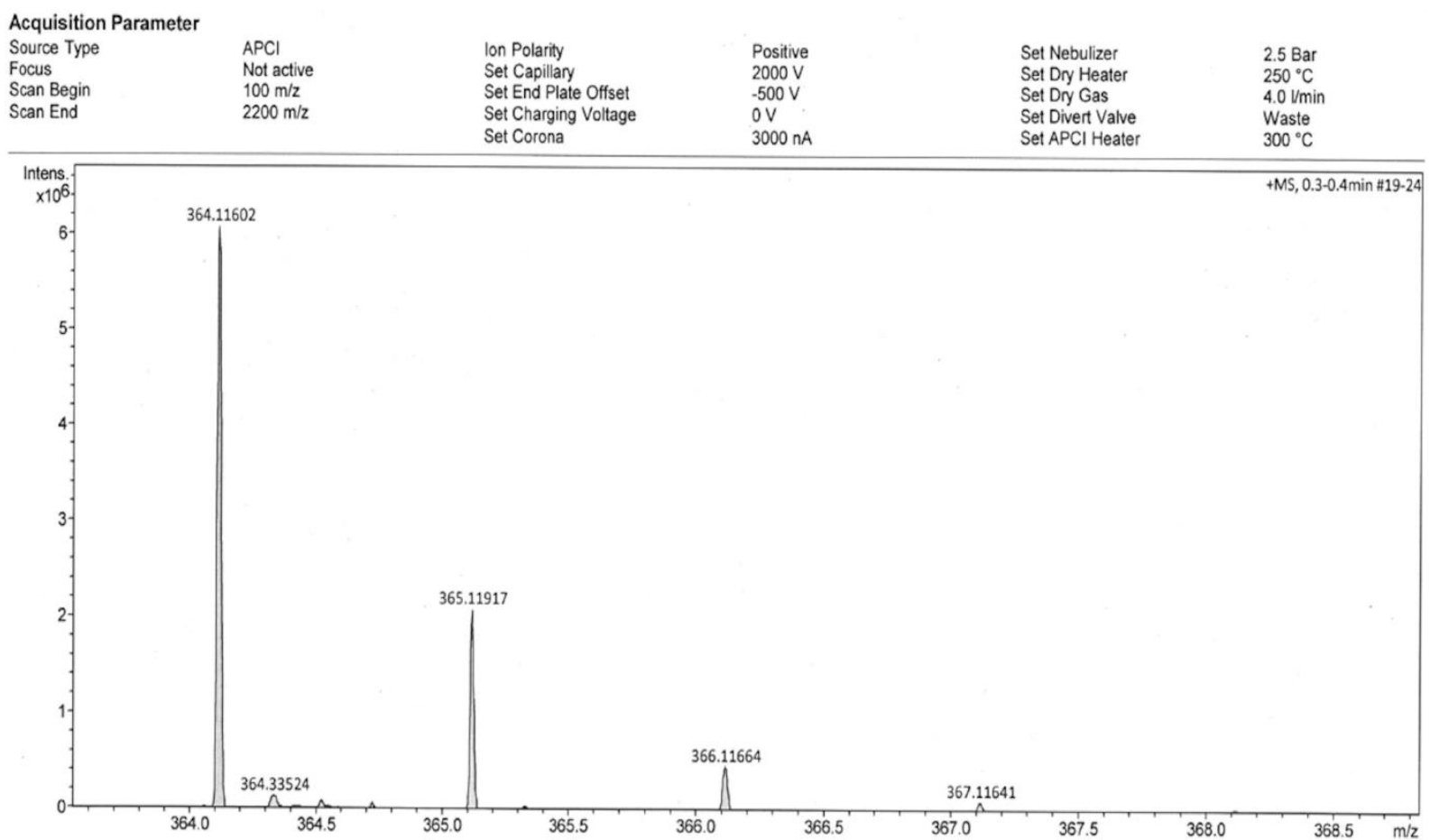

Figure S157. HRMS (APCI) of 7. 


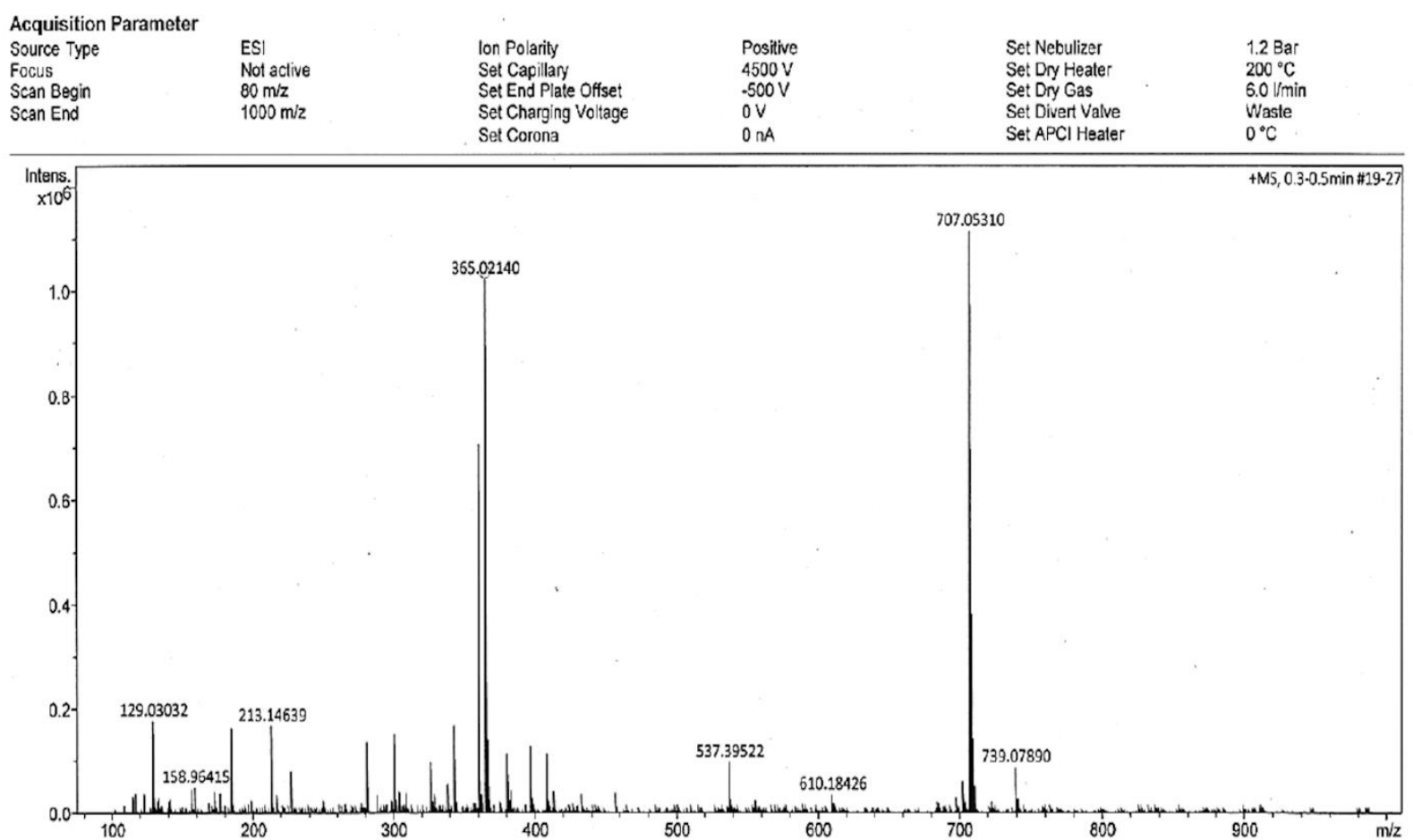

Figure S158. HRMS (ESI) of 9.

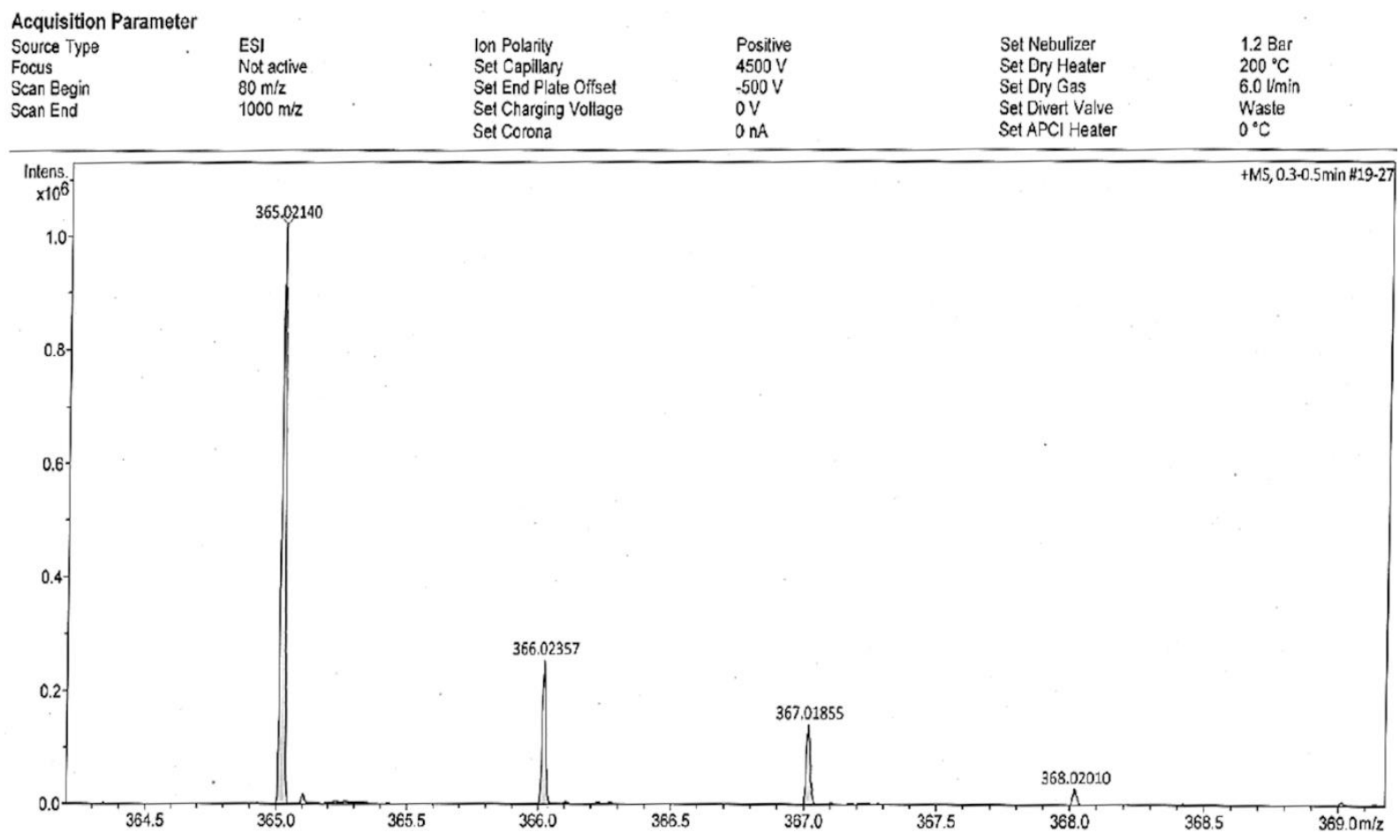

Figure S159. HRMS (ESI) of 9. 


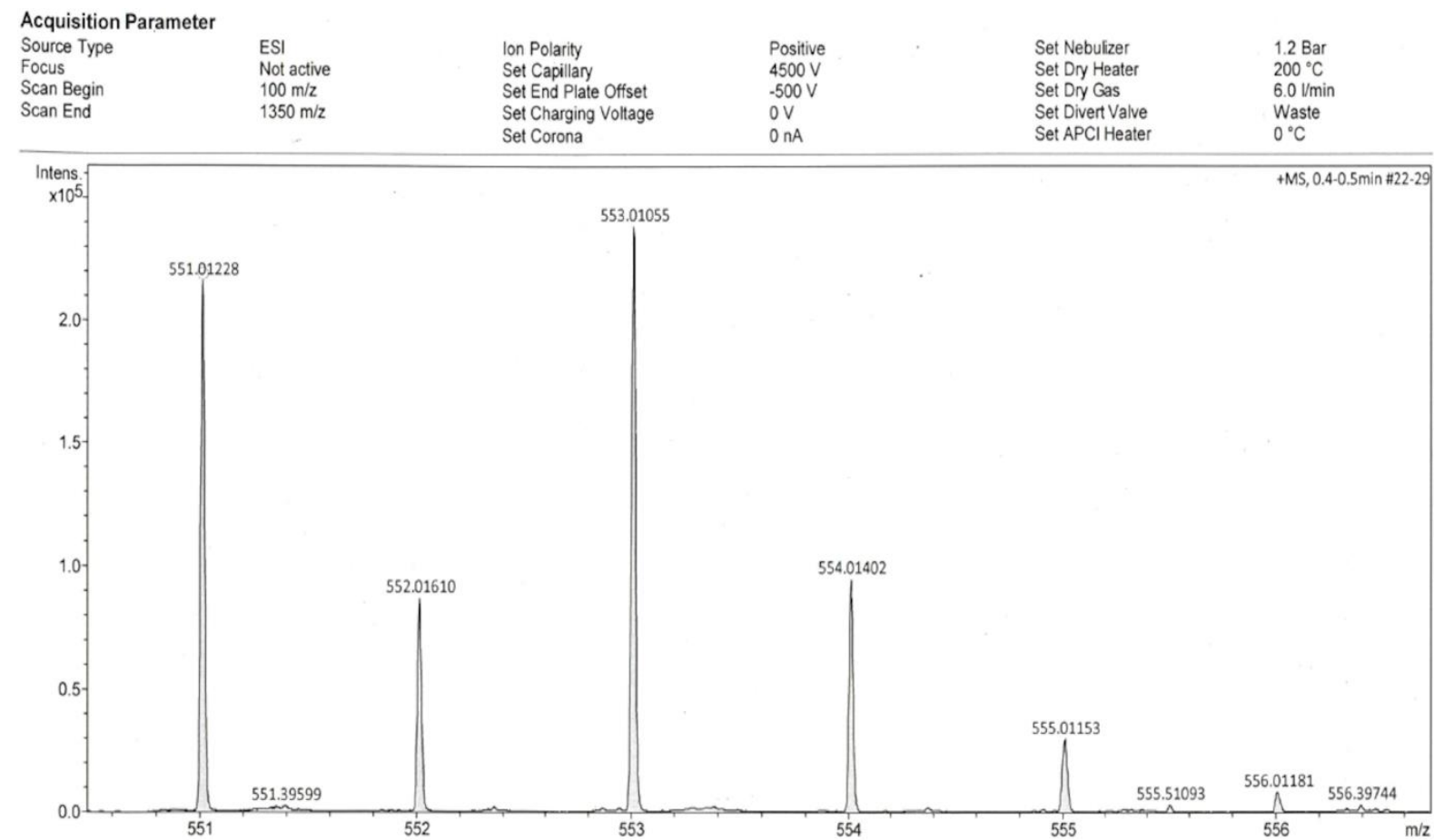

Figure S160. HRMS (ESI) of 11a.

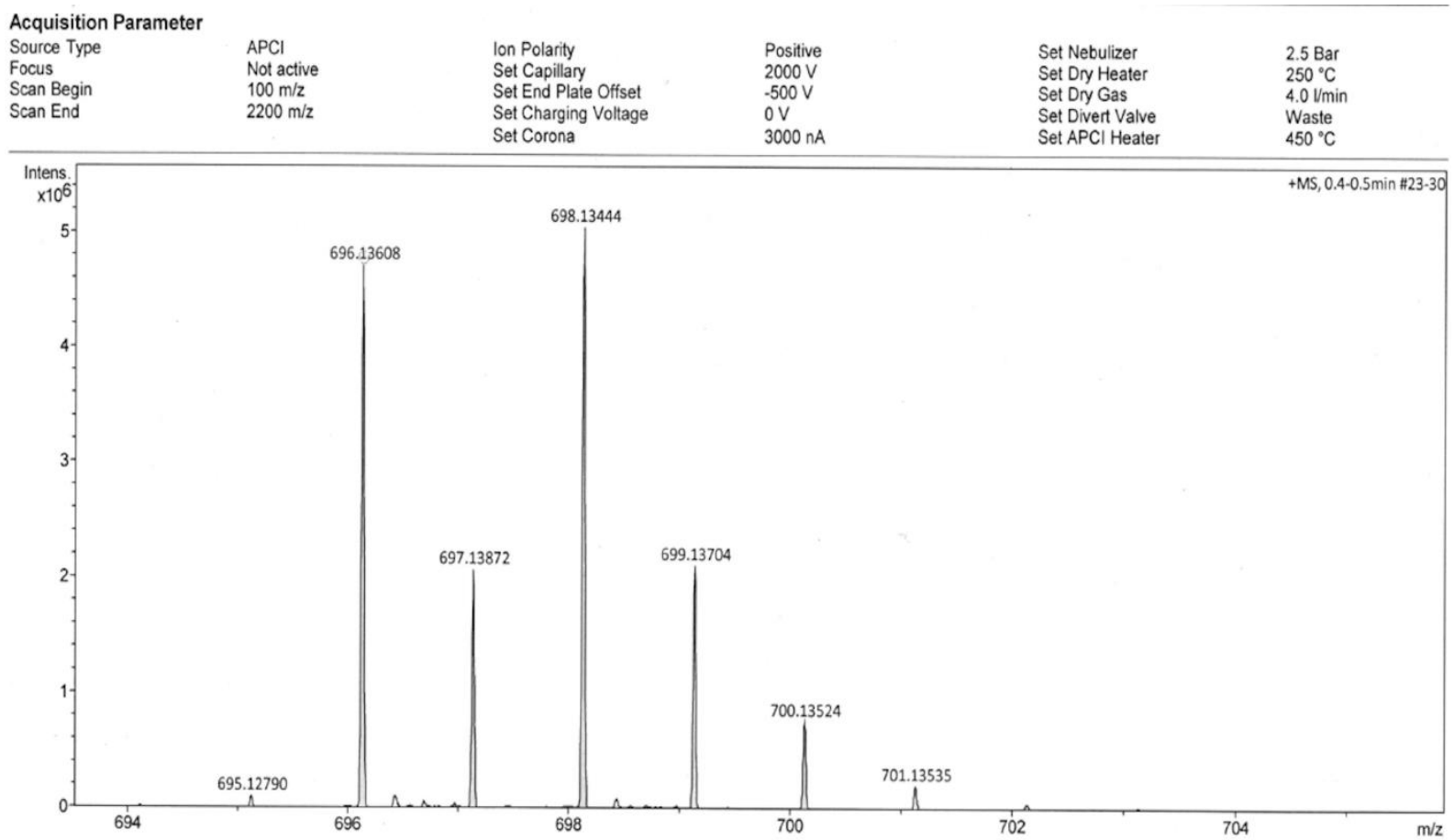

Figure S161. HRMS (APCI) of 11b. 
Acquisition Parameter

\begin{tabular}{|c|c|c|c|c|c|}
\hline \multirow{5}{*}{$\begin{array}{l}\text { Source Type } \\
\text { Focus } \\
\text { Scan Begin } \\
\text { Scan End }\end{array}$} & ESI & Ion Polarity & Positive & Set Nebulizer & $1.2 \mathrm{Bar}$ \\
\hline & Not active & Sel Capillary & $4500 \mathrm{~V}$ & Set Dy Healer & $200^{\circ} \mathrm{C}$ \\
\hline & $80 \mathrm{~m} / \mathrm{z}$ & Set End Plate Oftset & $.500 \mathrm{~V}$ & Sel Dy Gas & $6.0 \mathrm{lmin}$ \\
\hline & $1000 \mathrm{~m} / \mathrm{z}$ & Sel Charging Voltage & OV & Set DivertValve & Waste \\
\hline & & Set Corona & $O \cap A$ & Set APCI Heater & ${ }^{\circ} \mathrm{C}$ \\
\hline
\end{tabular}

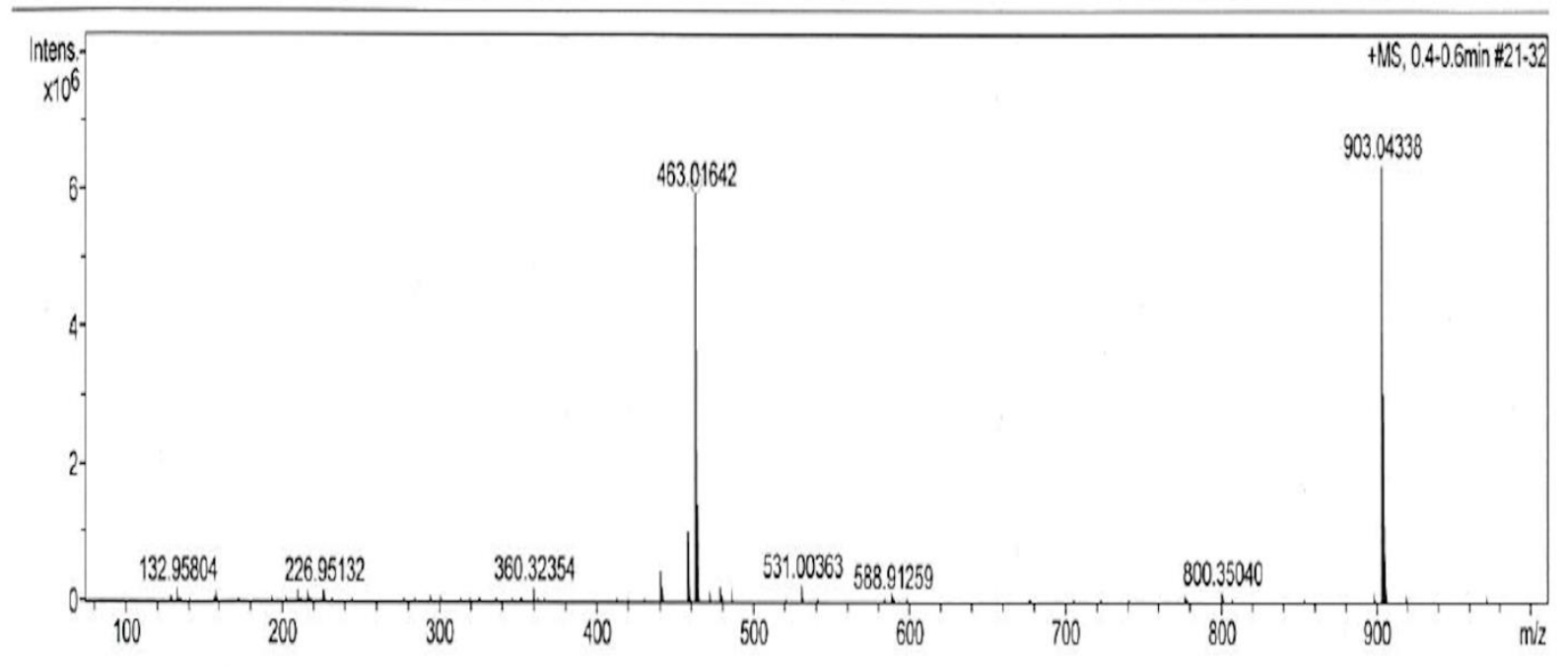

Figure S162. HRMS (ESI) of 13(S)b.

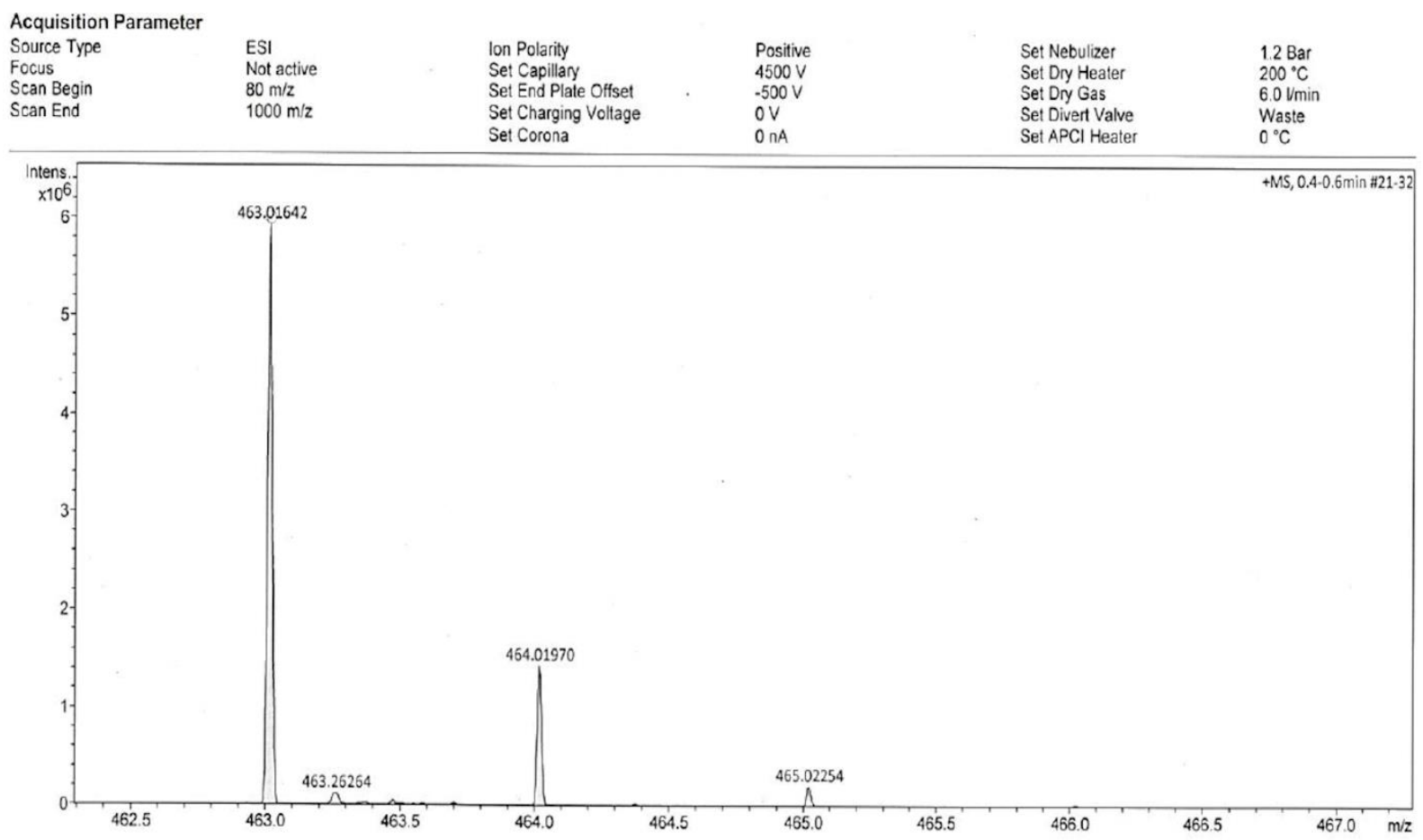

Figure S163. HRMS (ESI) of 13(S)b. 


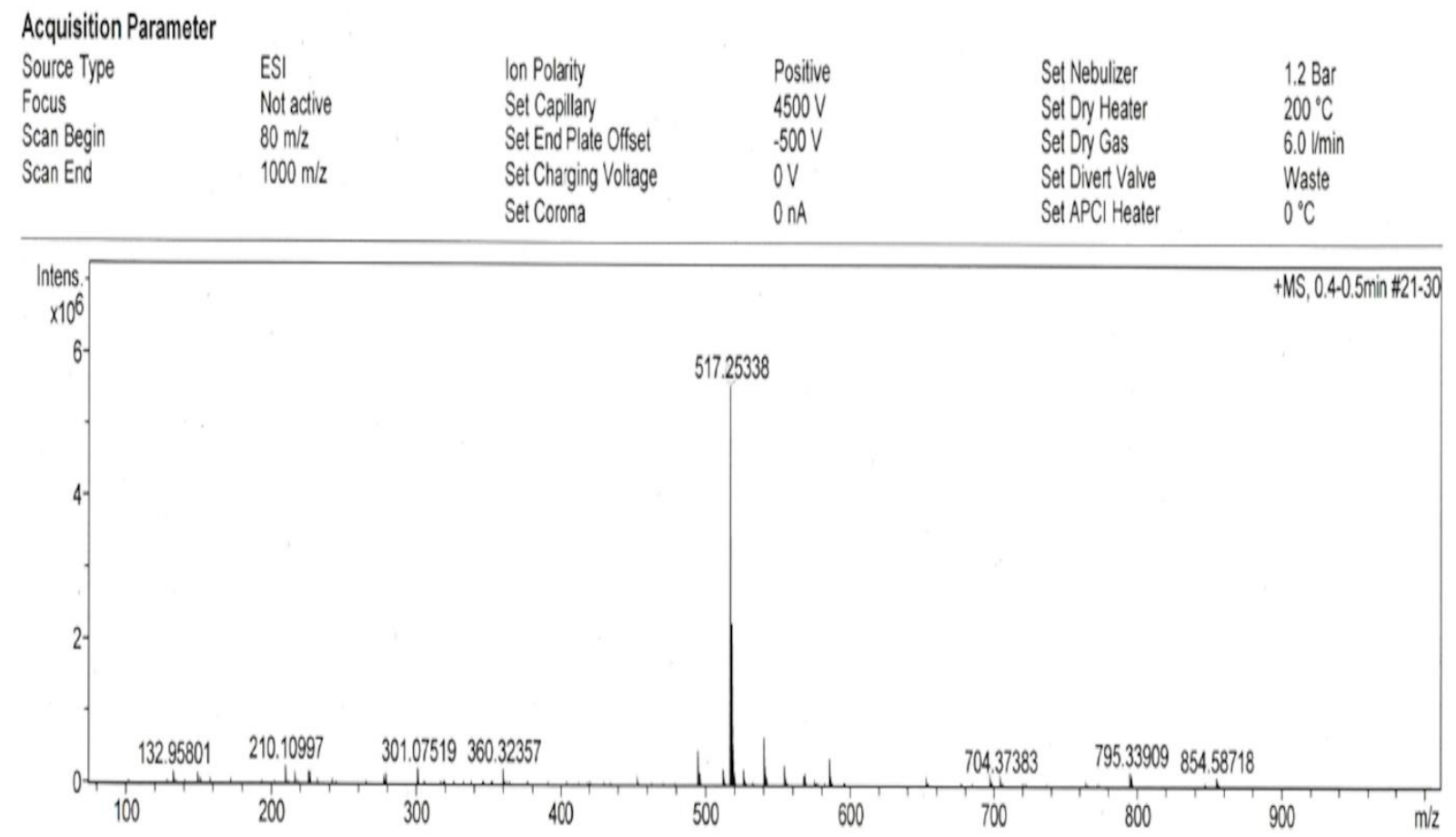

Figure S164. HRMS (ESI) of 14(S).

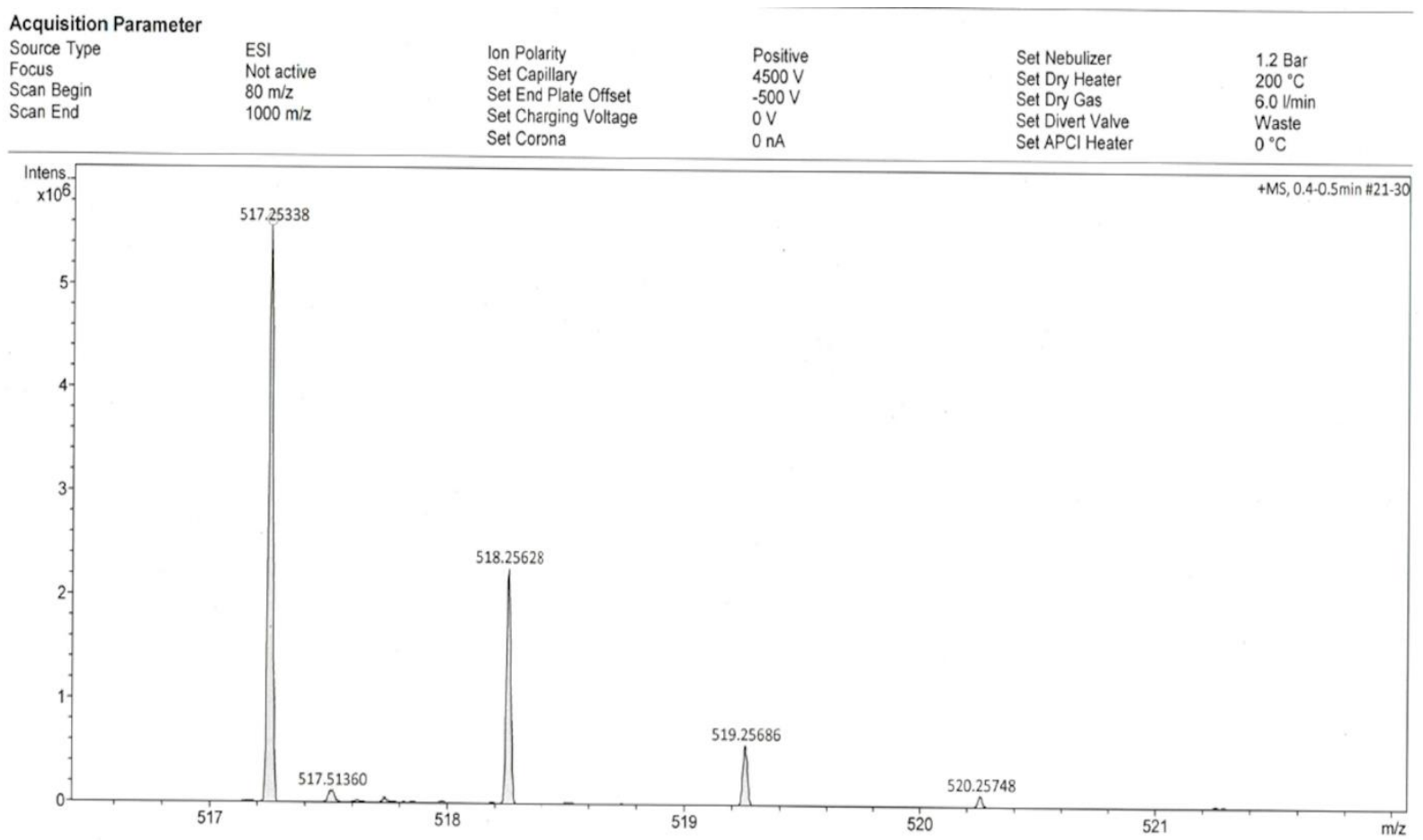

Figure S165. HRMS (ESI) of 14(S). 


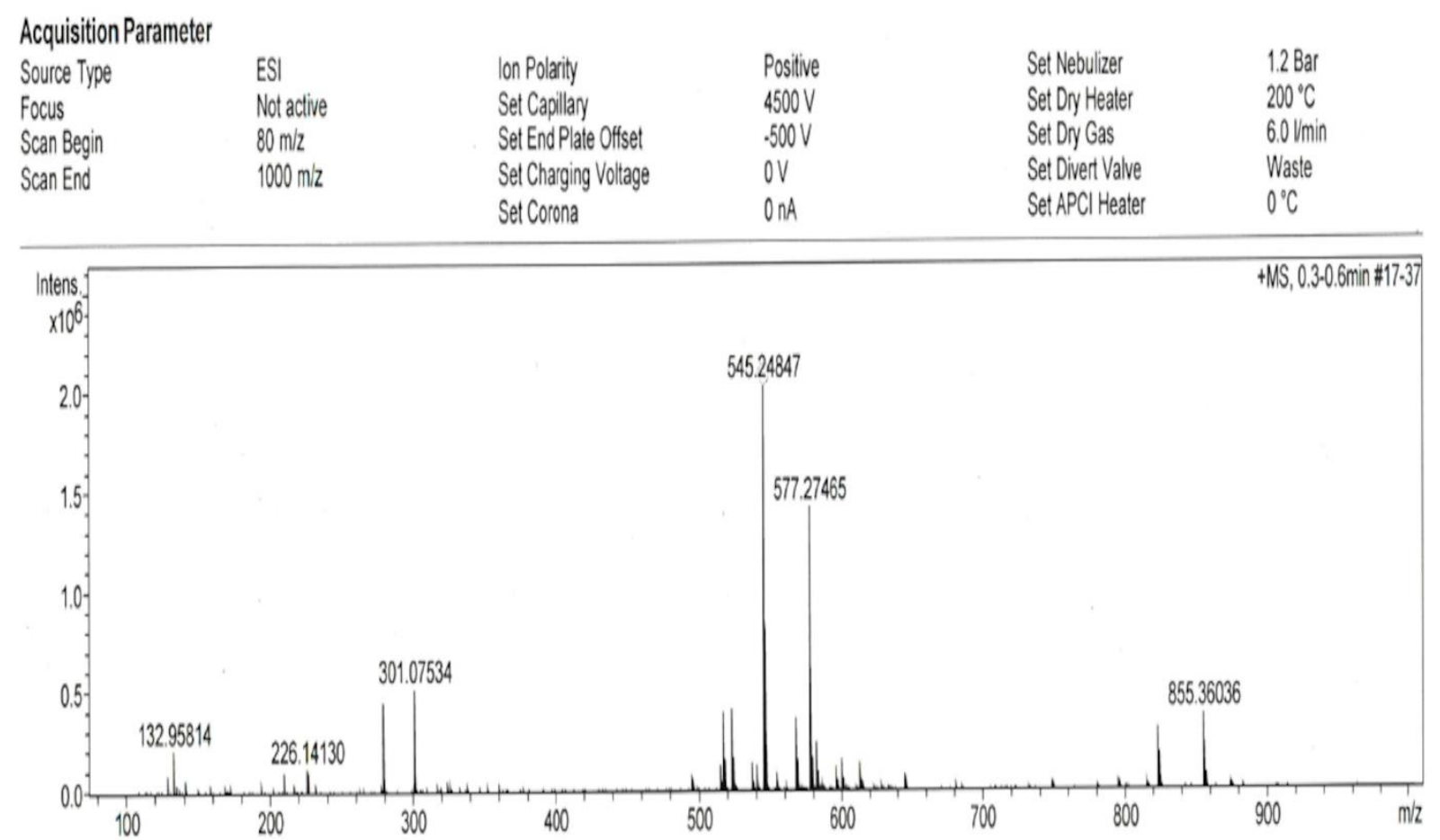

Figure S166. HRMS (ESI) of 15(S).

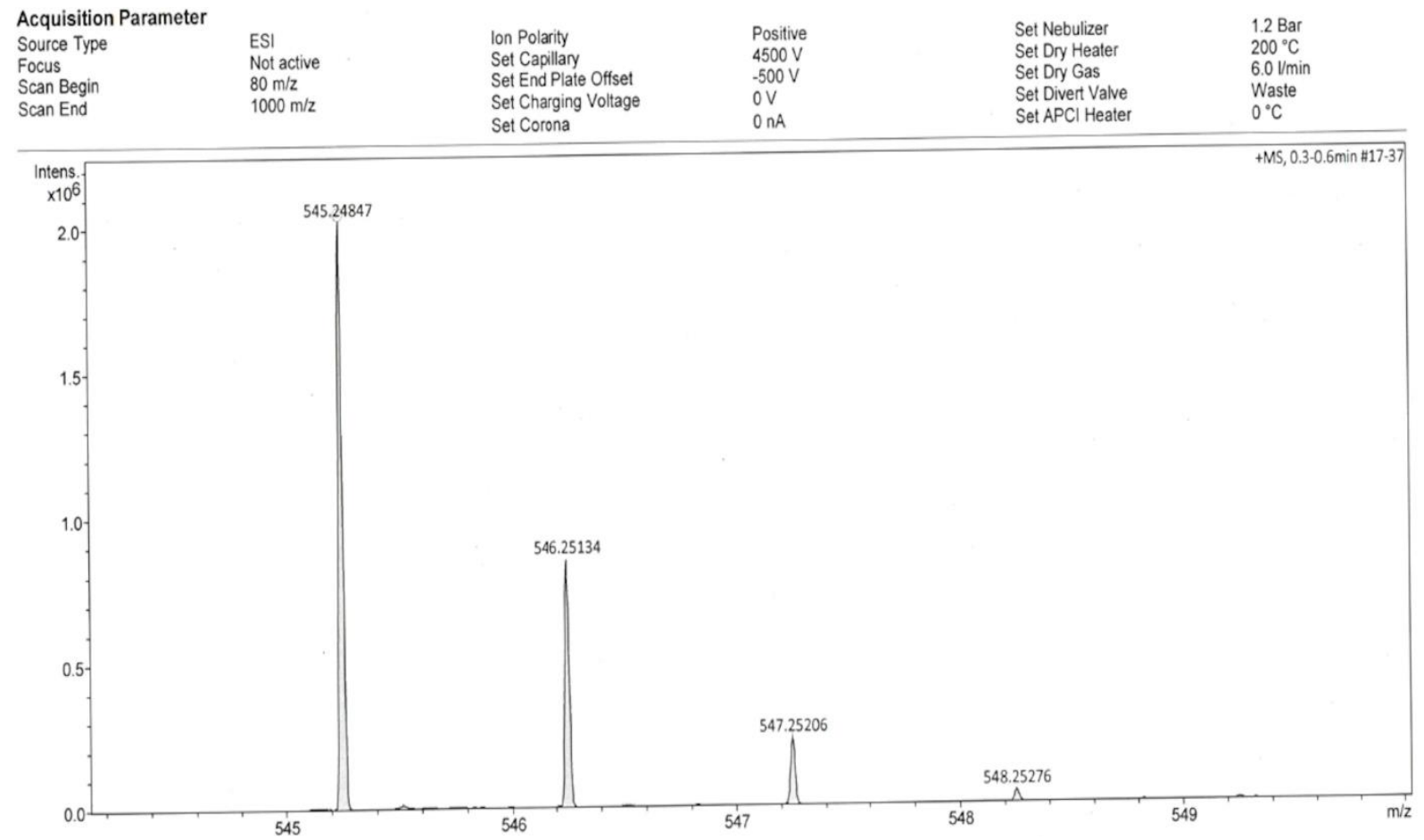

Figure S167. HRMS (ESI) of 15(S). 


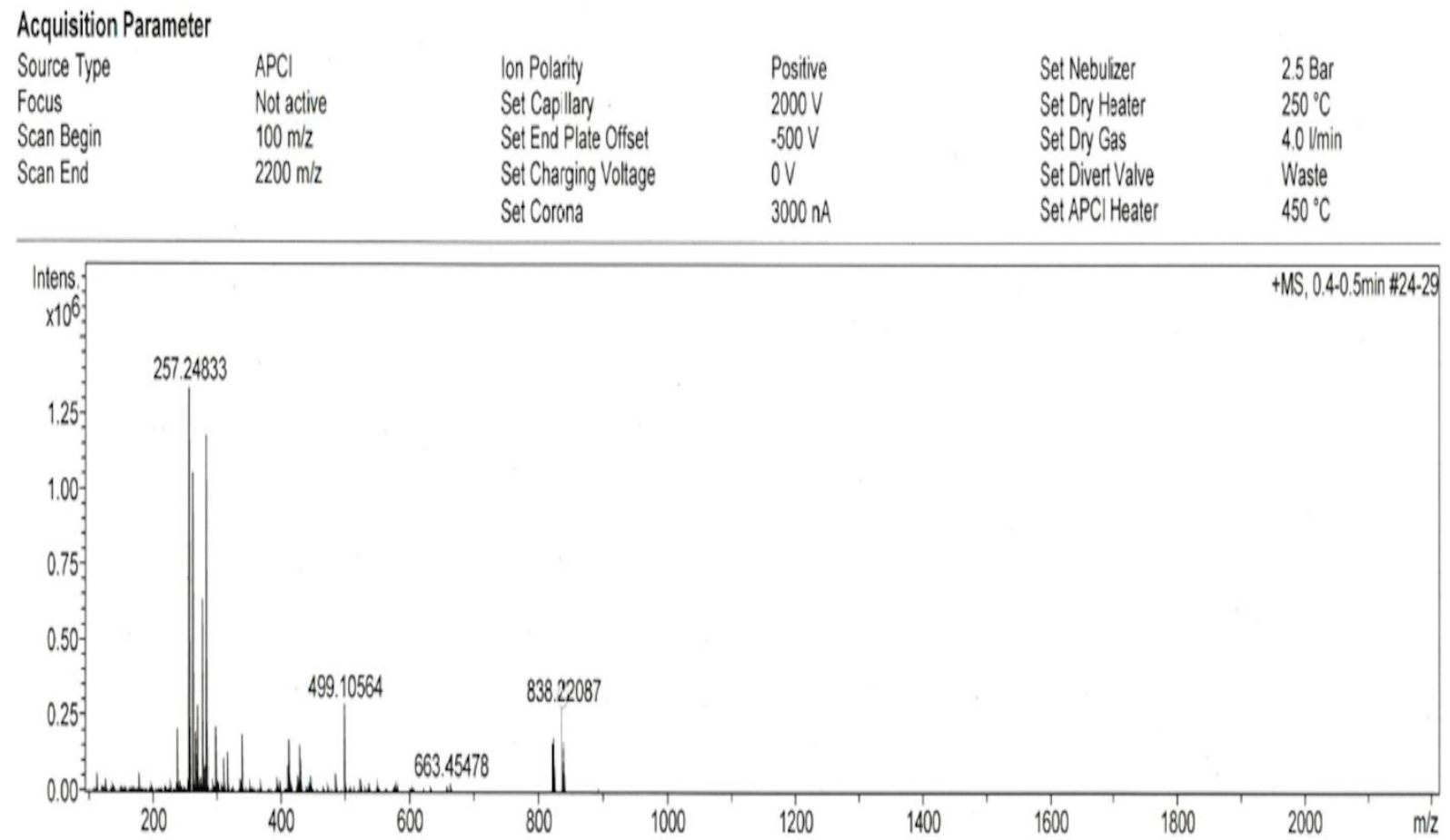

Figure S168. HRMS (APCI) of 17(S)a.

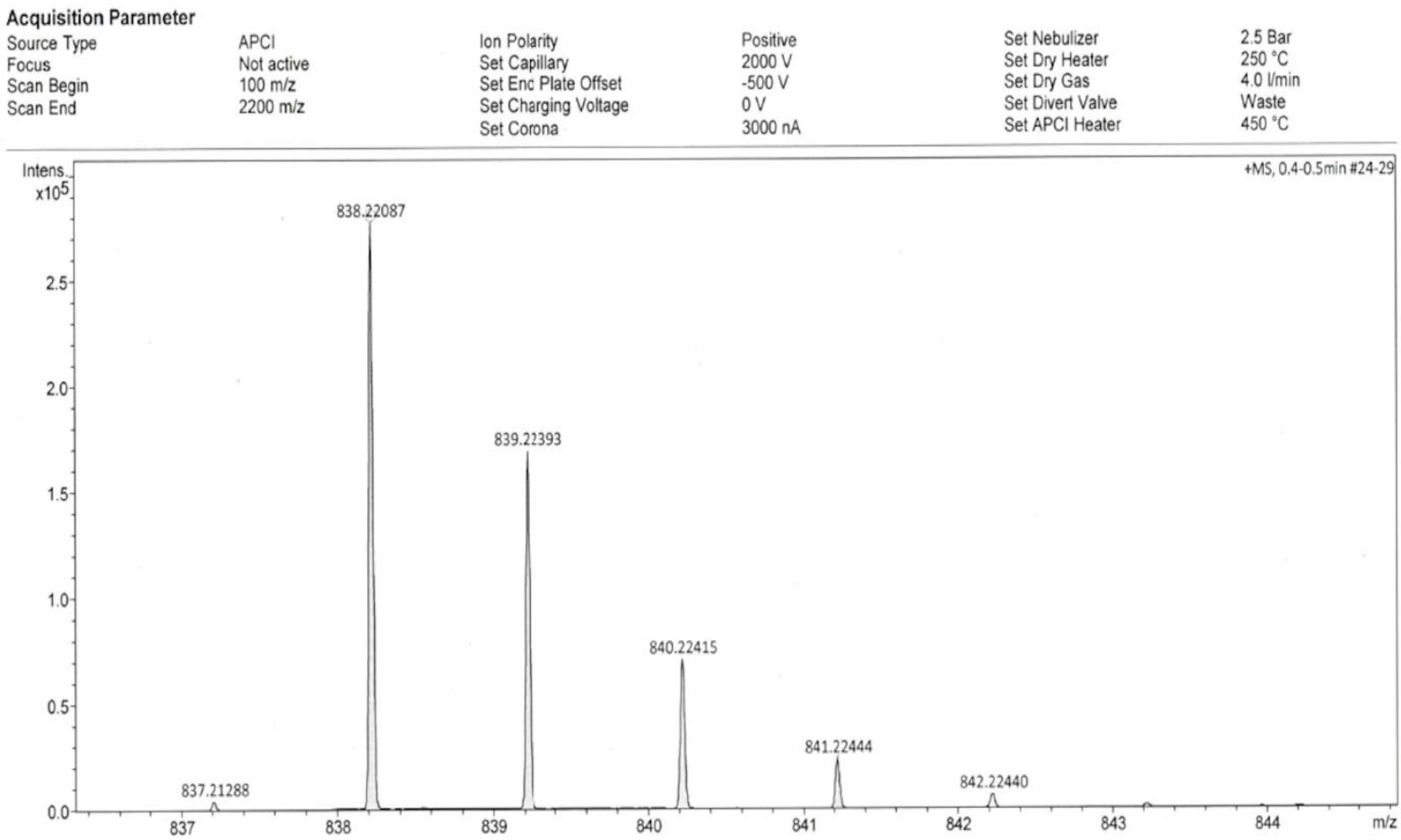

Figure S169. HRMS (APCI) of 17(S)a. 


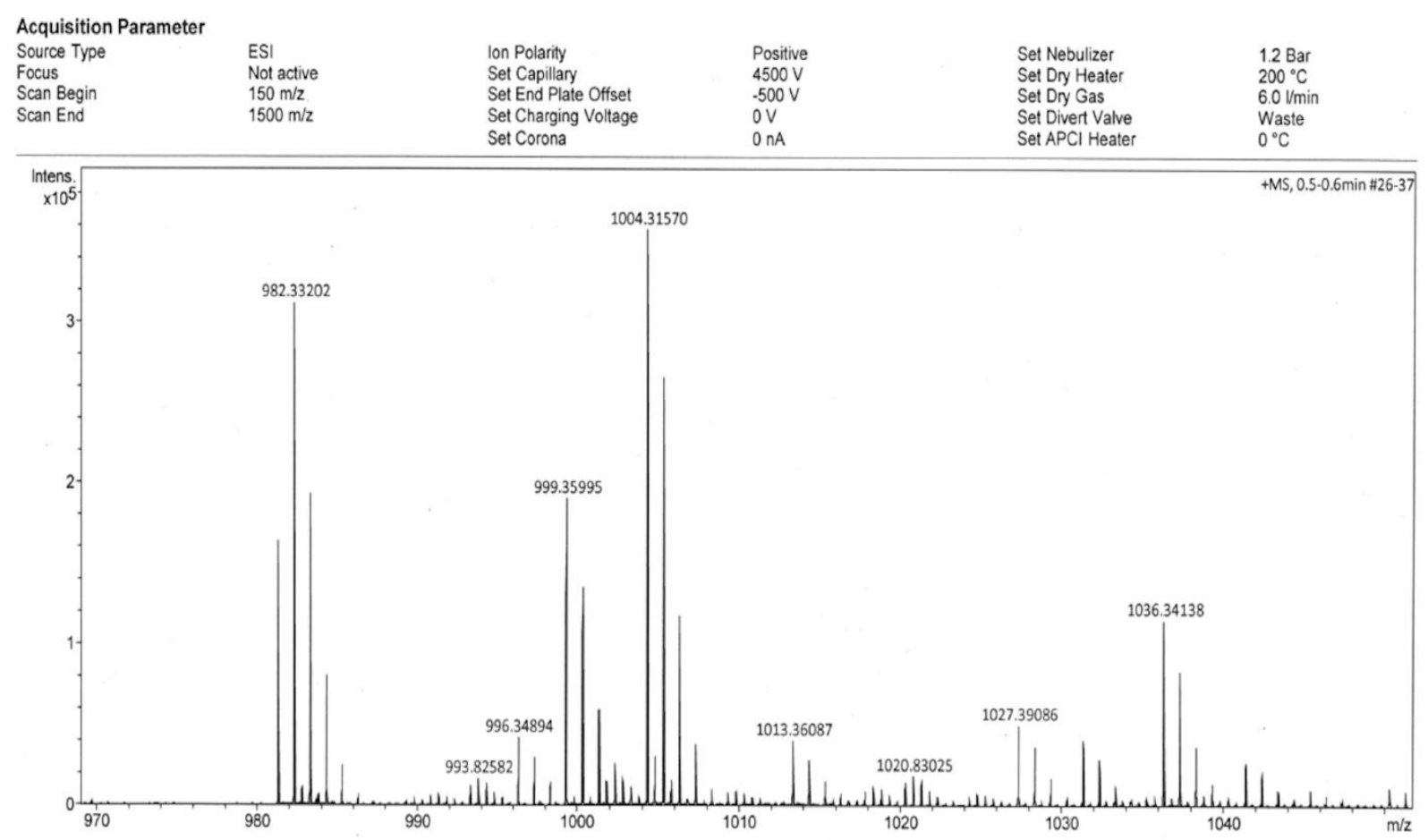

Figure S170. HRMS (ESI) of 17(S)b.

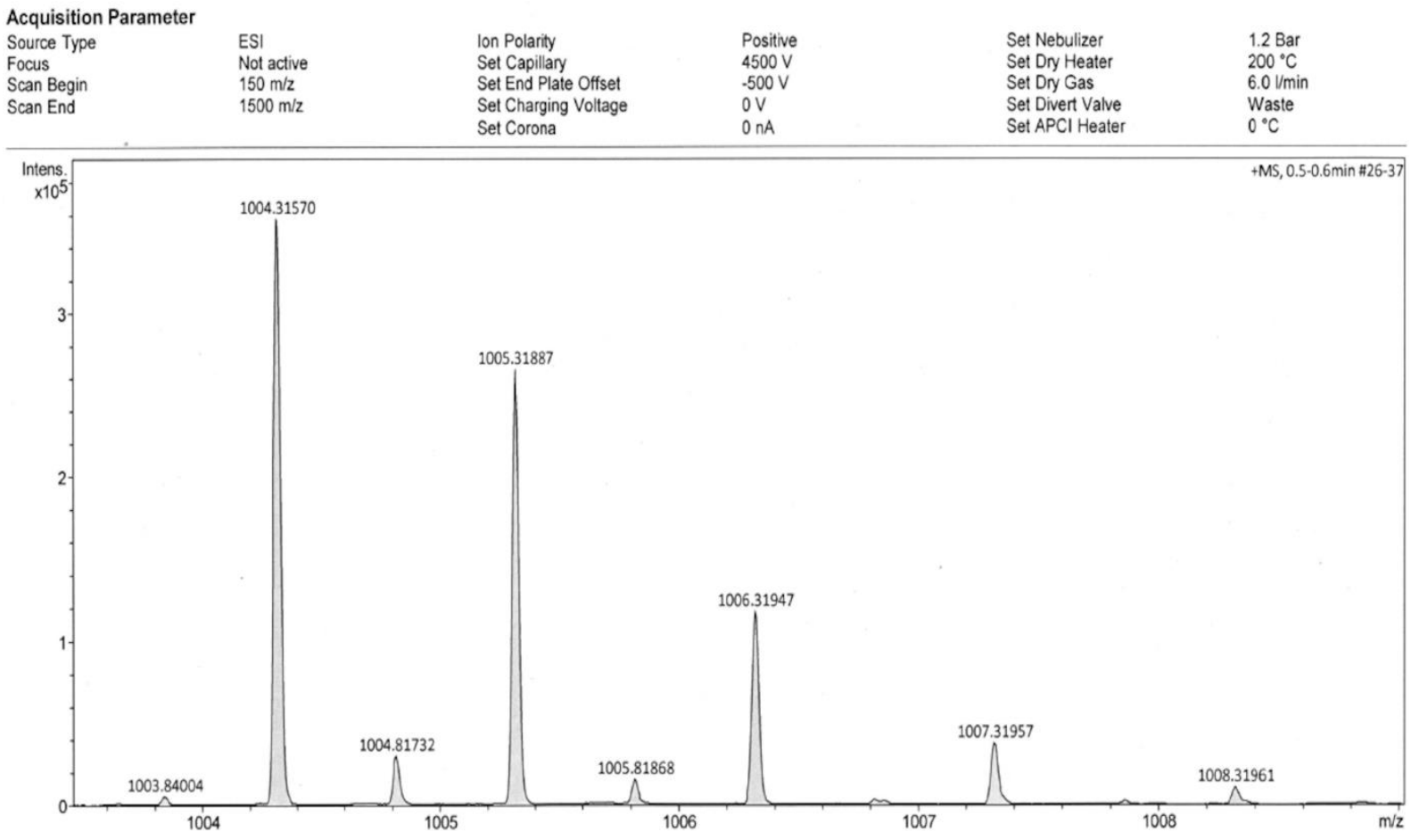

Figure S171. HRMS (ESI) of 17(S)b. 


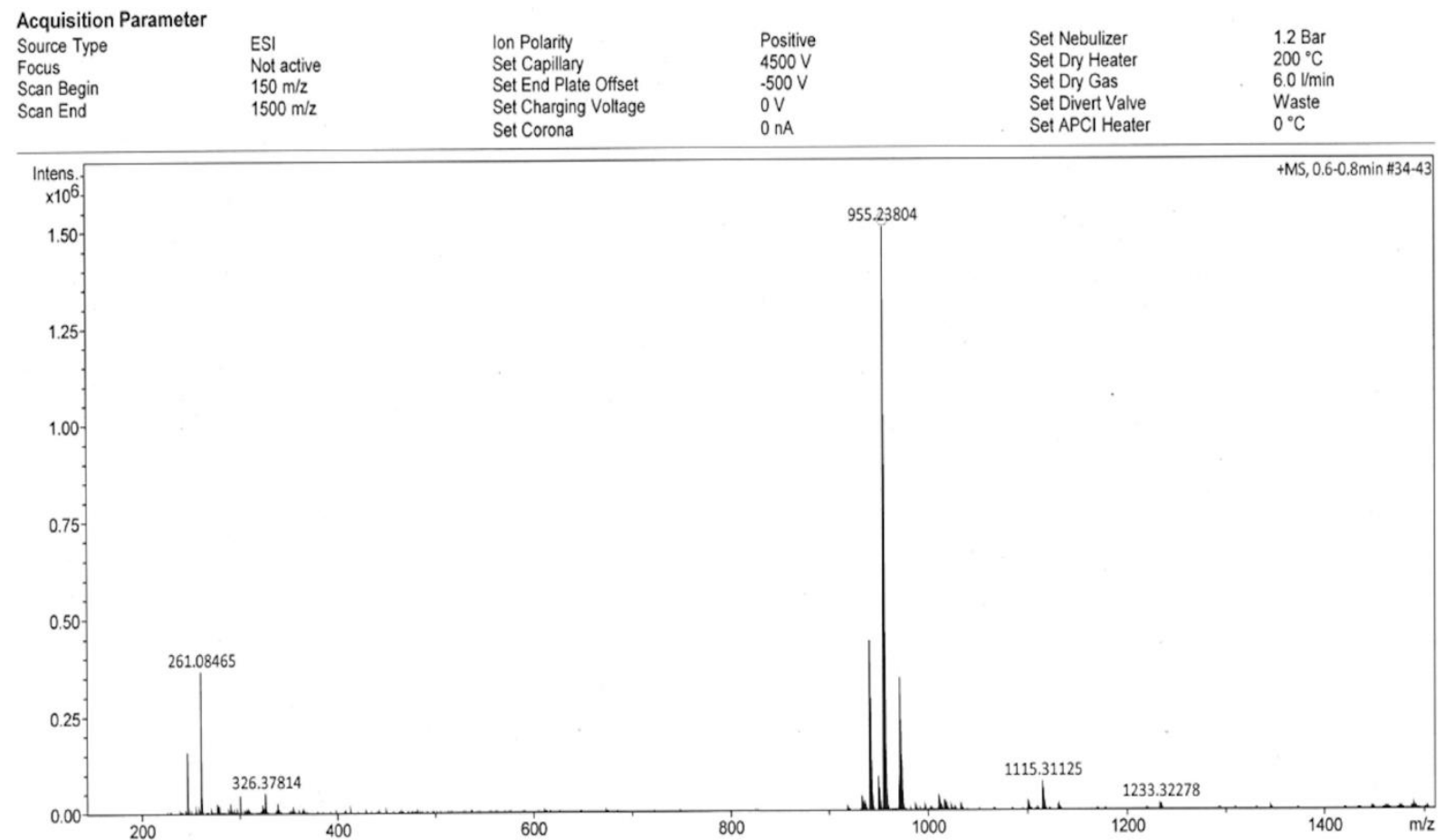

Figure S172. HRMS (ESI) of 18(S)a.

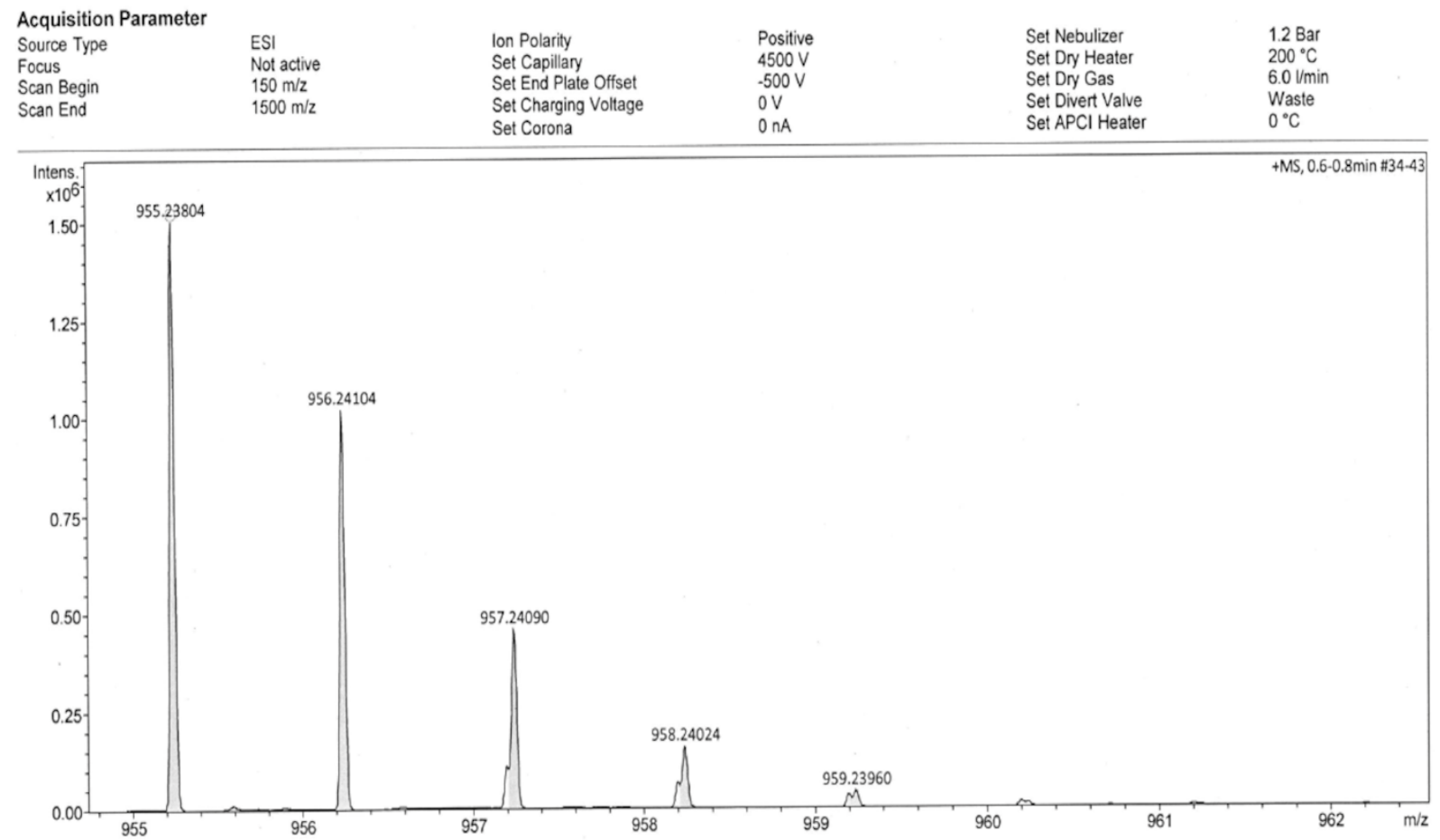

Figure S173. HRMS (ESI) of 18(S)a. 


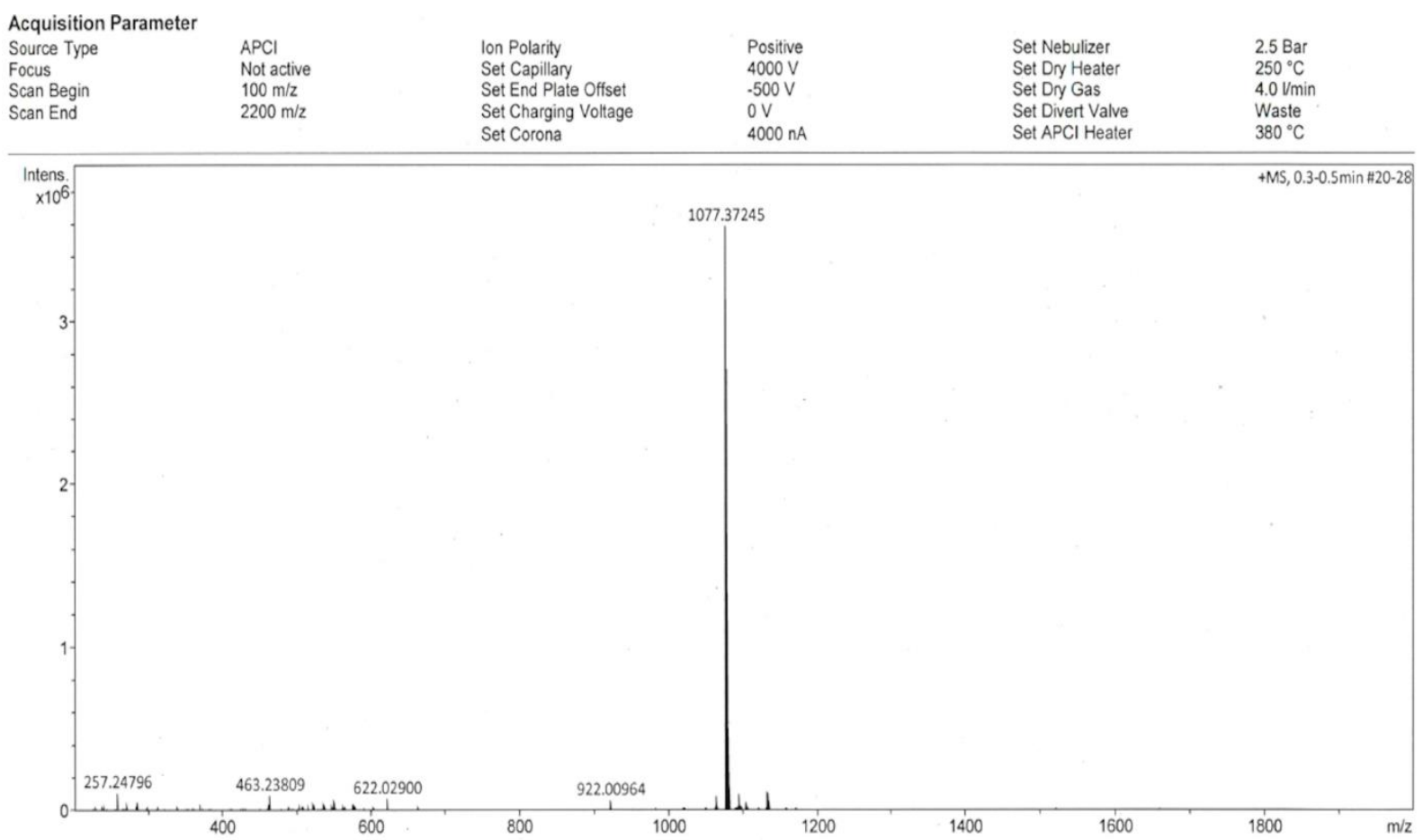

Figure S174. HRMS (APCI) of 18(S)b.

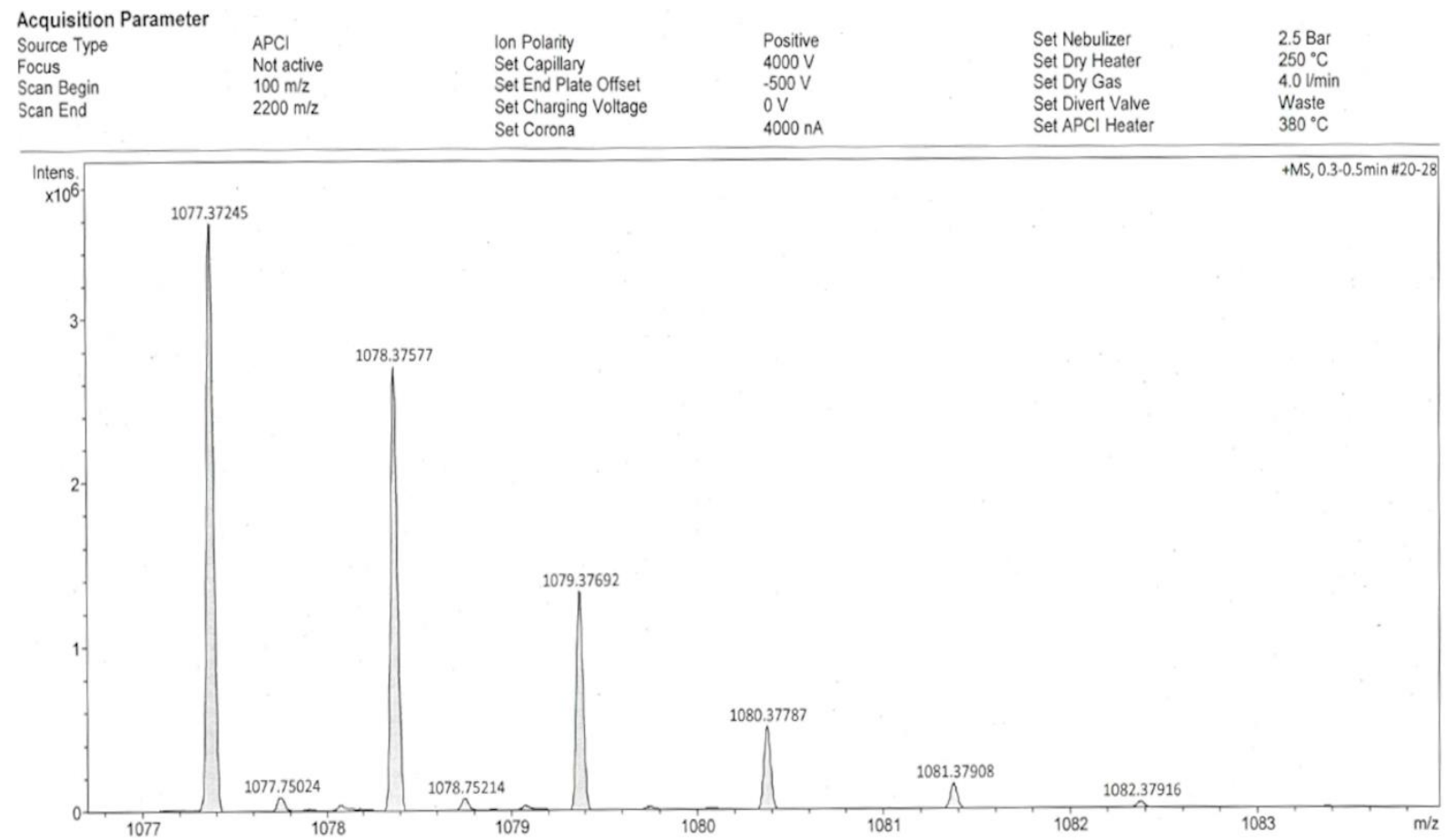

Figure S175. HRMS (APCI) of 18(S)b. 


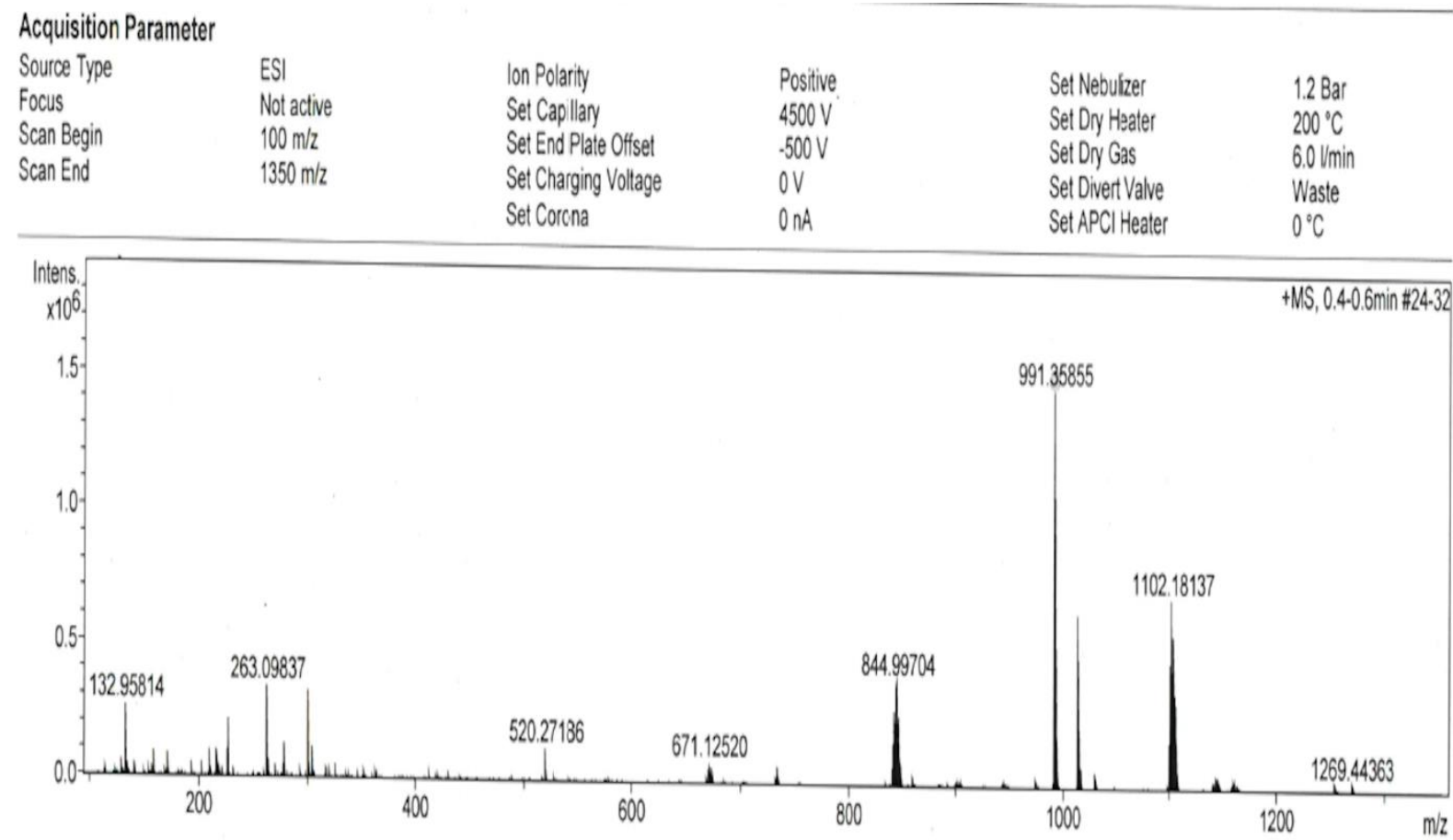

Figure S176. HRMS (ESI) of 19(S)a.

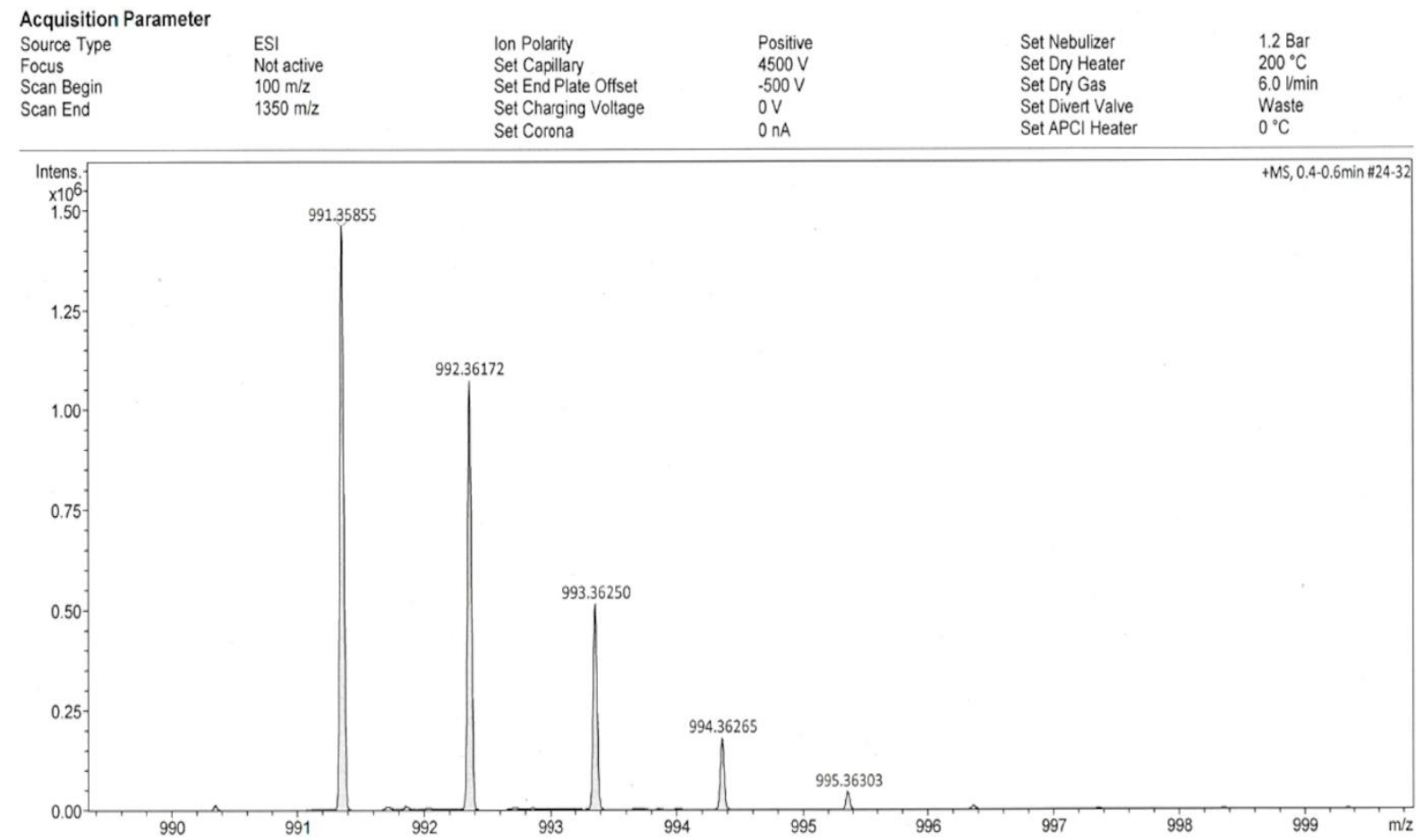

Figure S177. HRMS (ESI) of 19(S)a. 


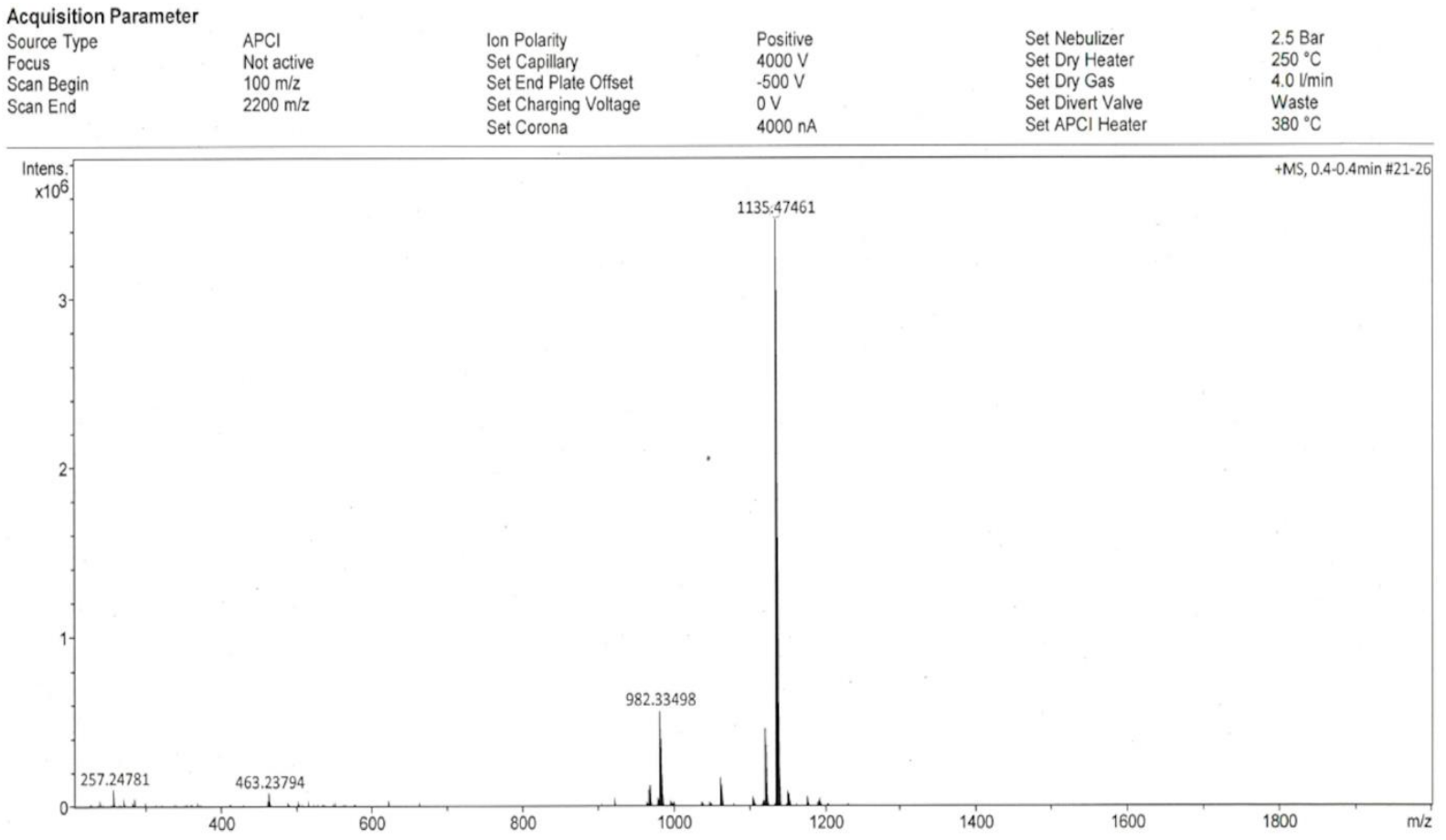

Figure S178. HRMS (APCI) of 19(S)b.

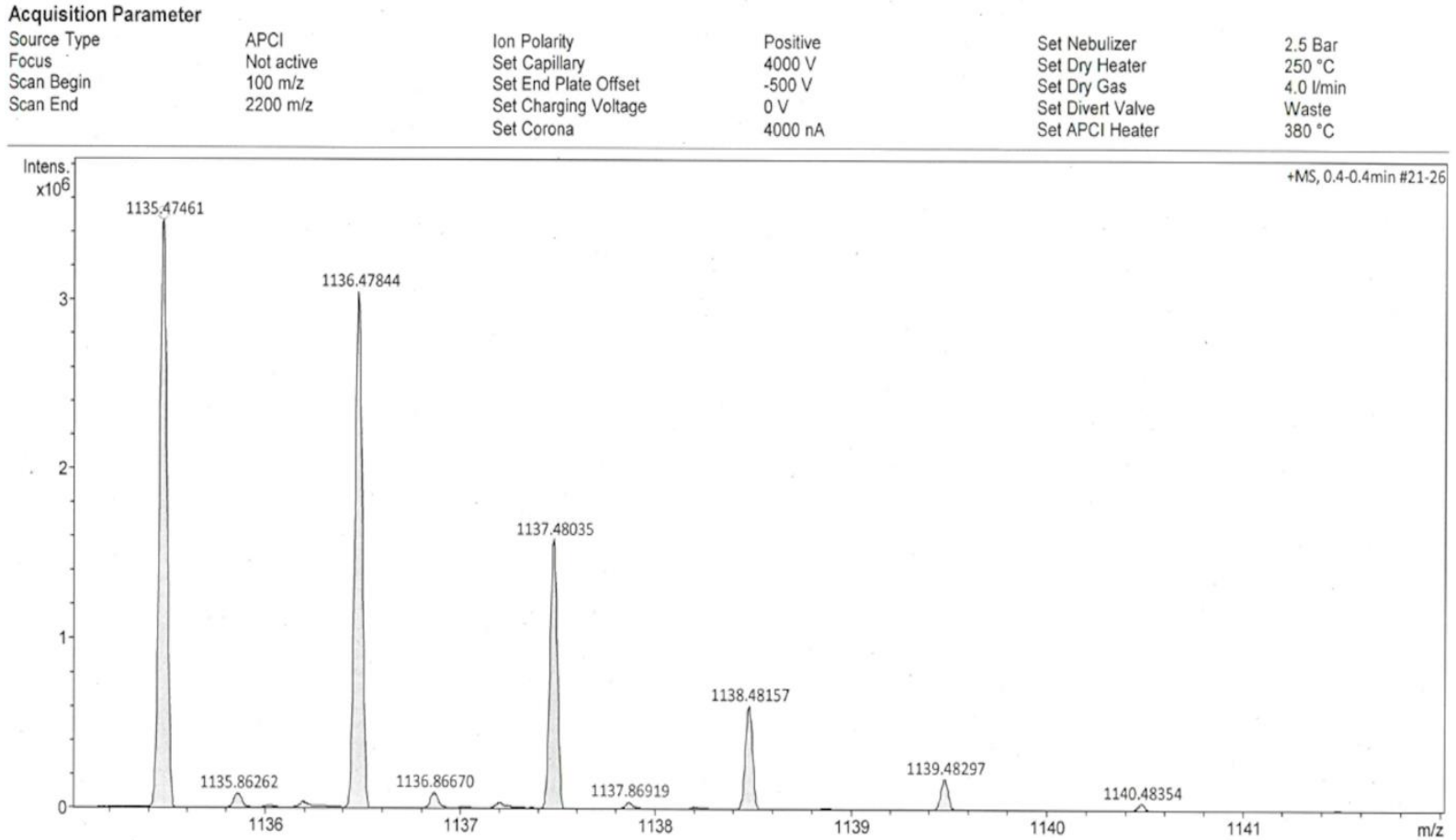

Figure S179. HRMS (APCI) of 19(S)b. 


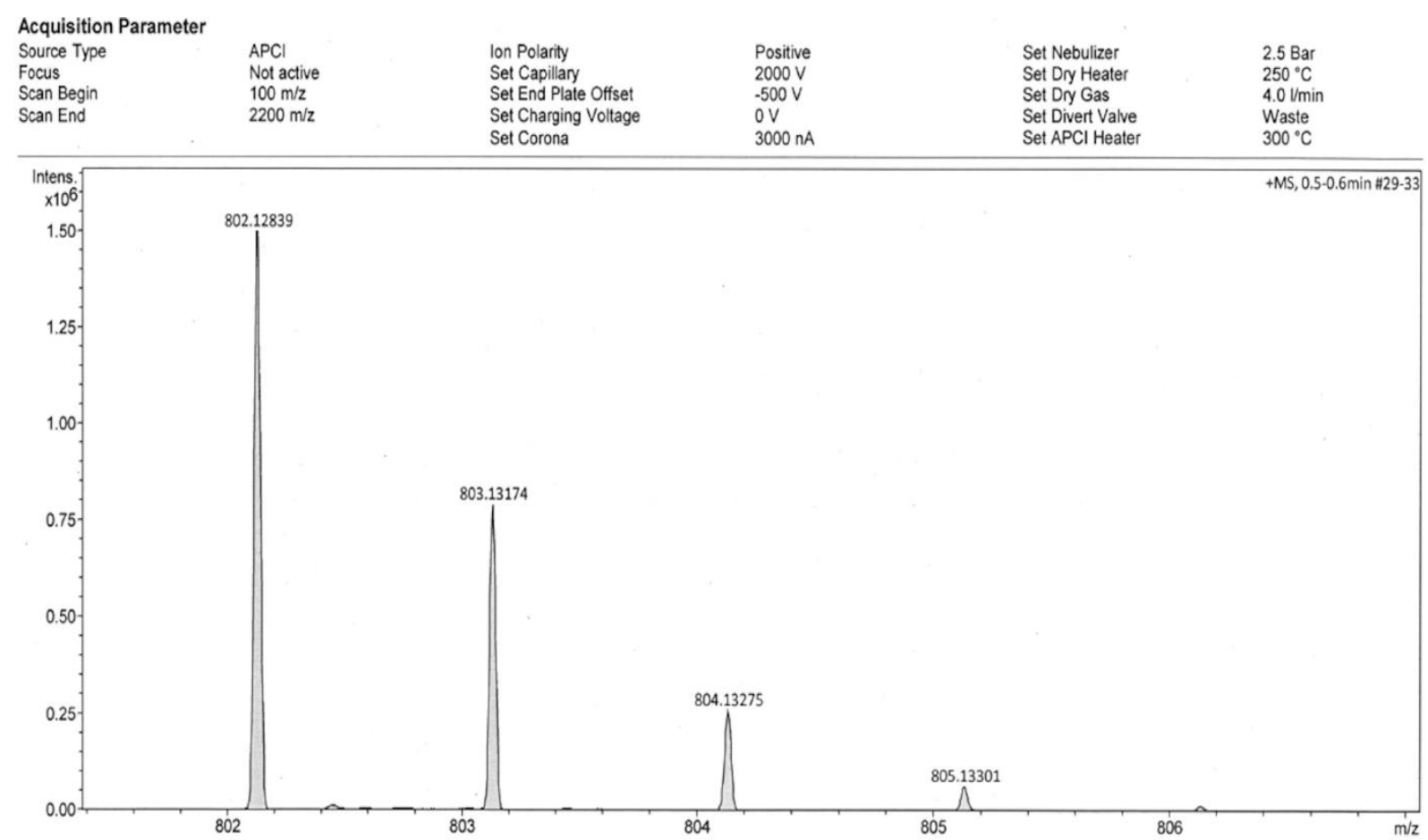

Figure S180. HRMS (APCI) of $\mathbf{2 0}(S)$.

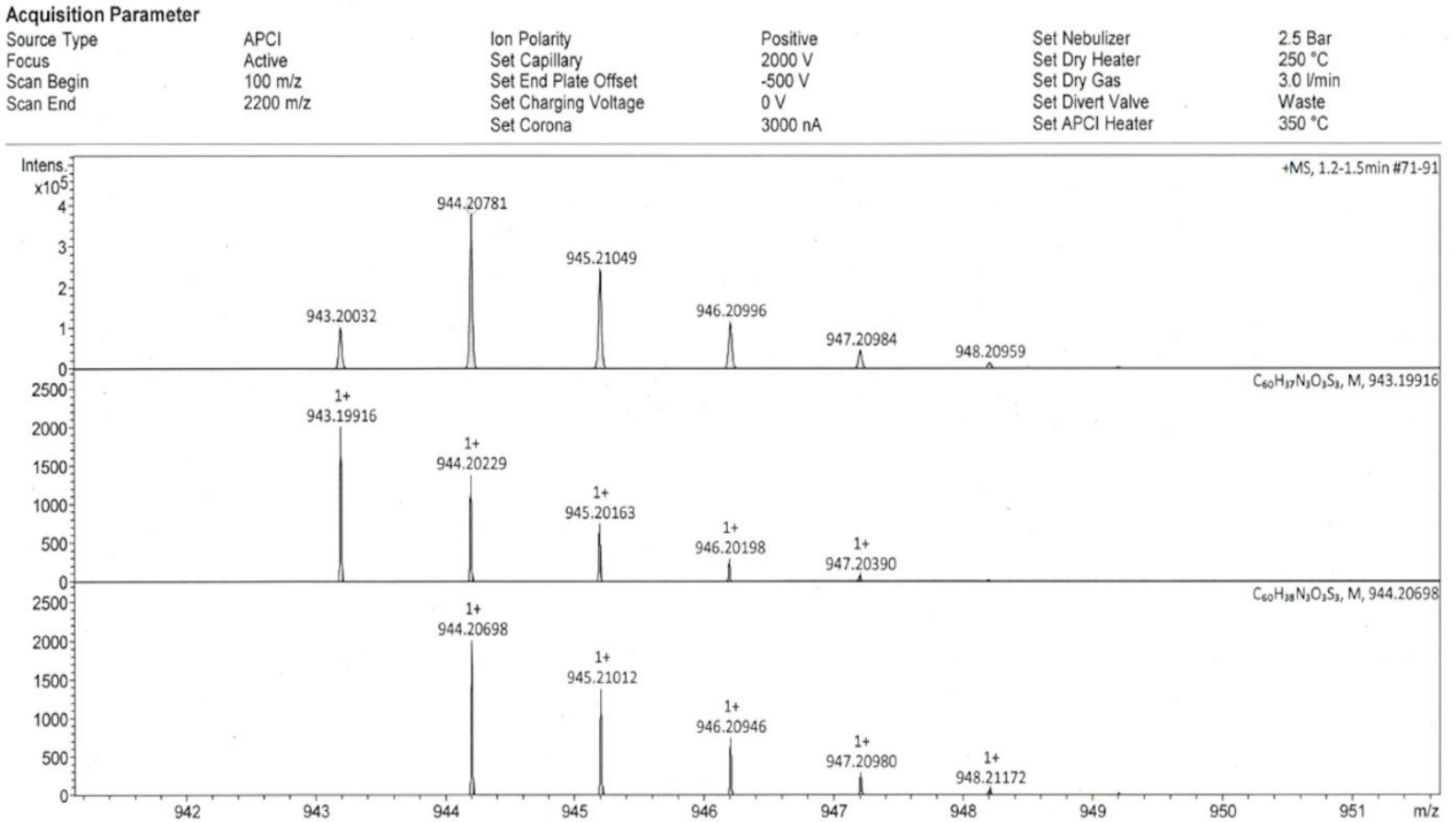

Figure S181. HRMS (APCI) of $\mathbf{2 1}(S)$. 


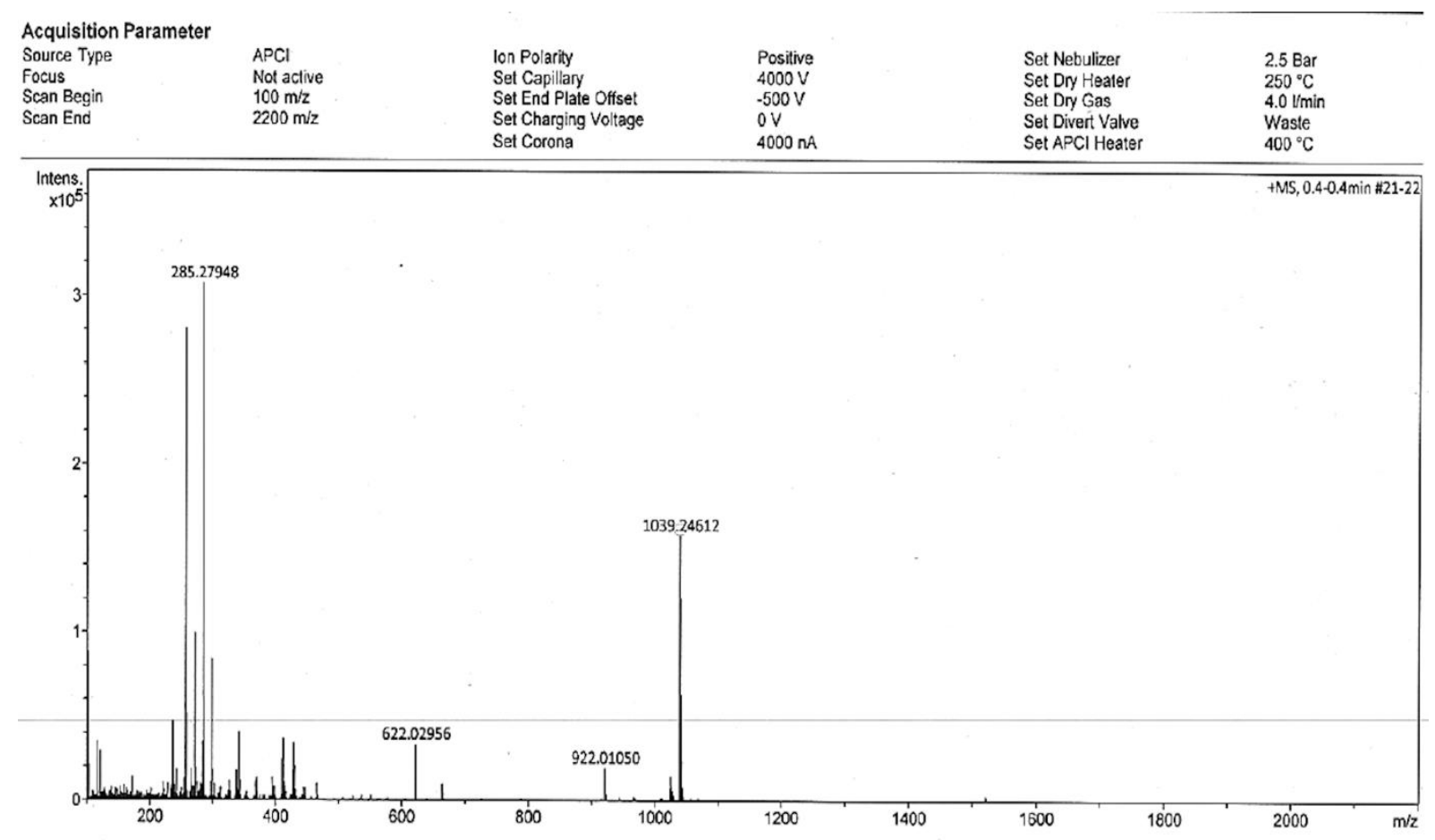

Figure S182. HRMS (APCI) of 22(S).

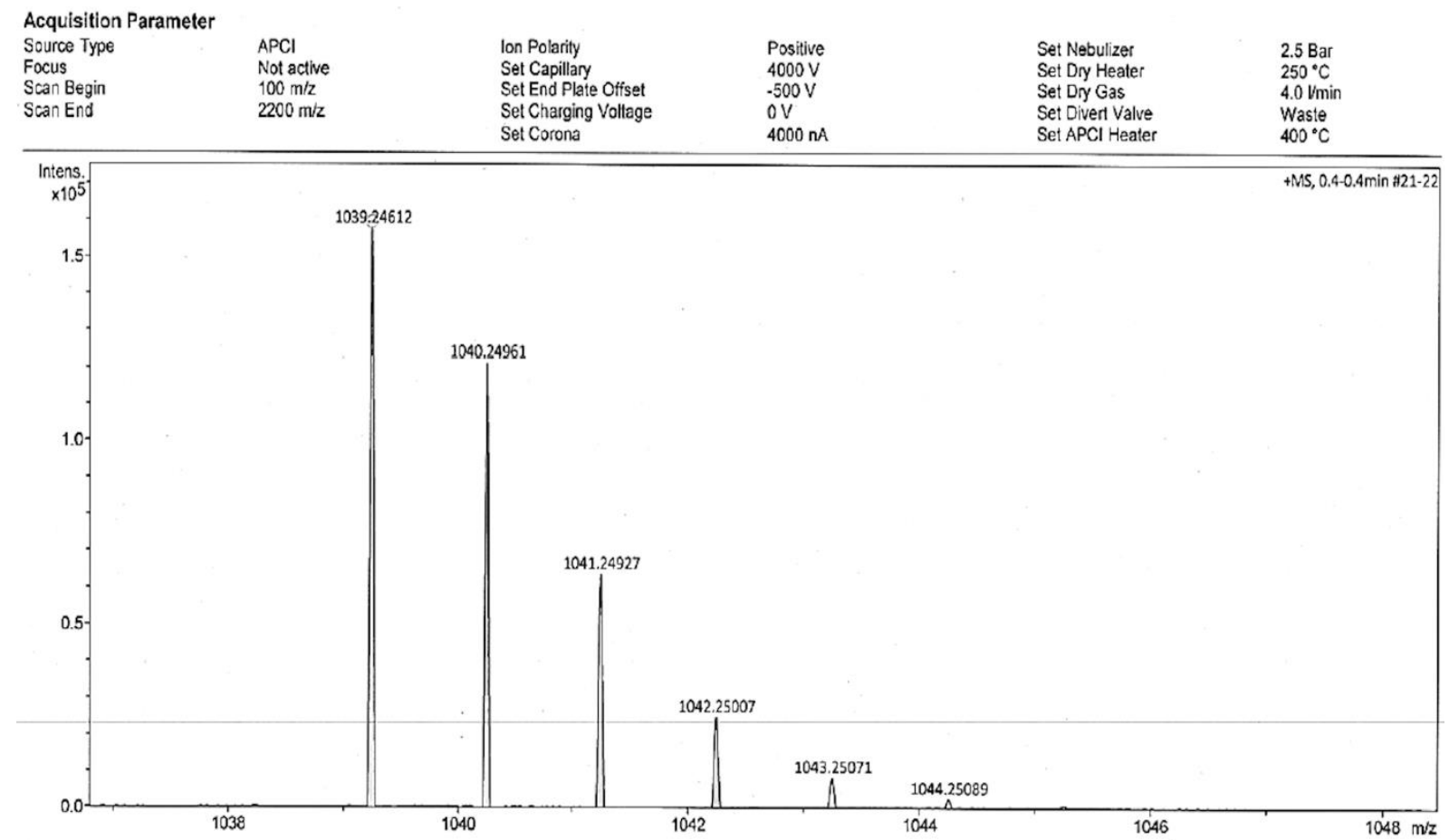

Figure S183. HRMS (APCI) of 22(S). 


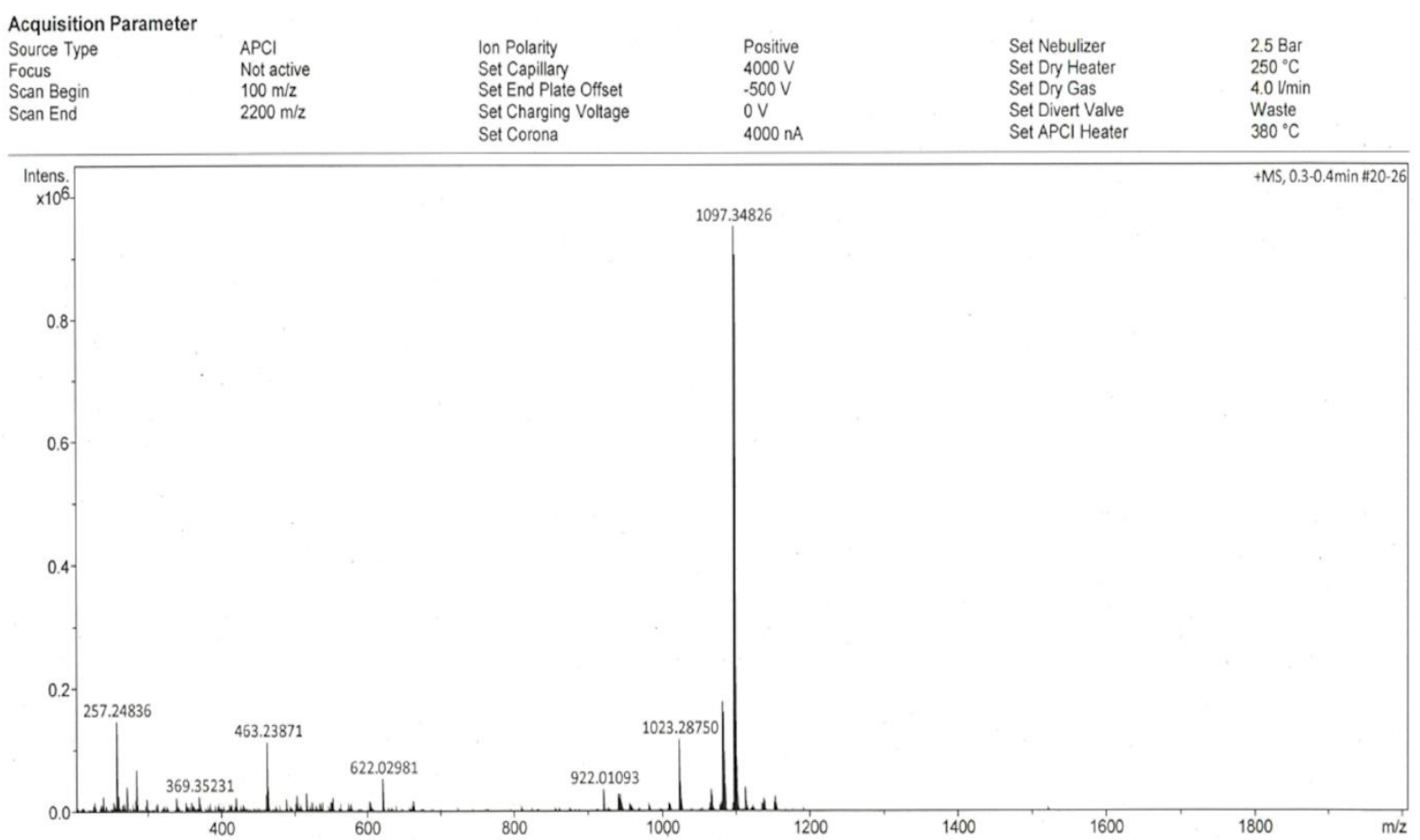

Figure S184. HRMS (APCI) of $\mathbf{2 3}(S)$.

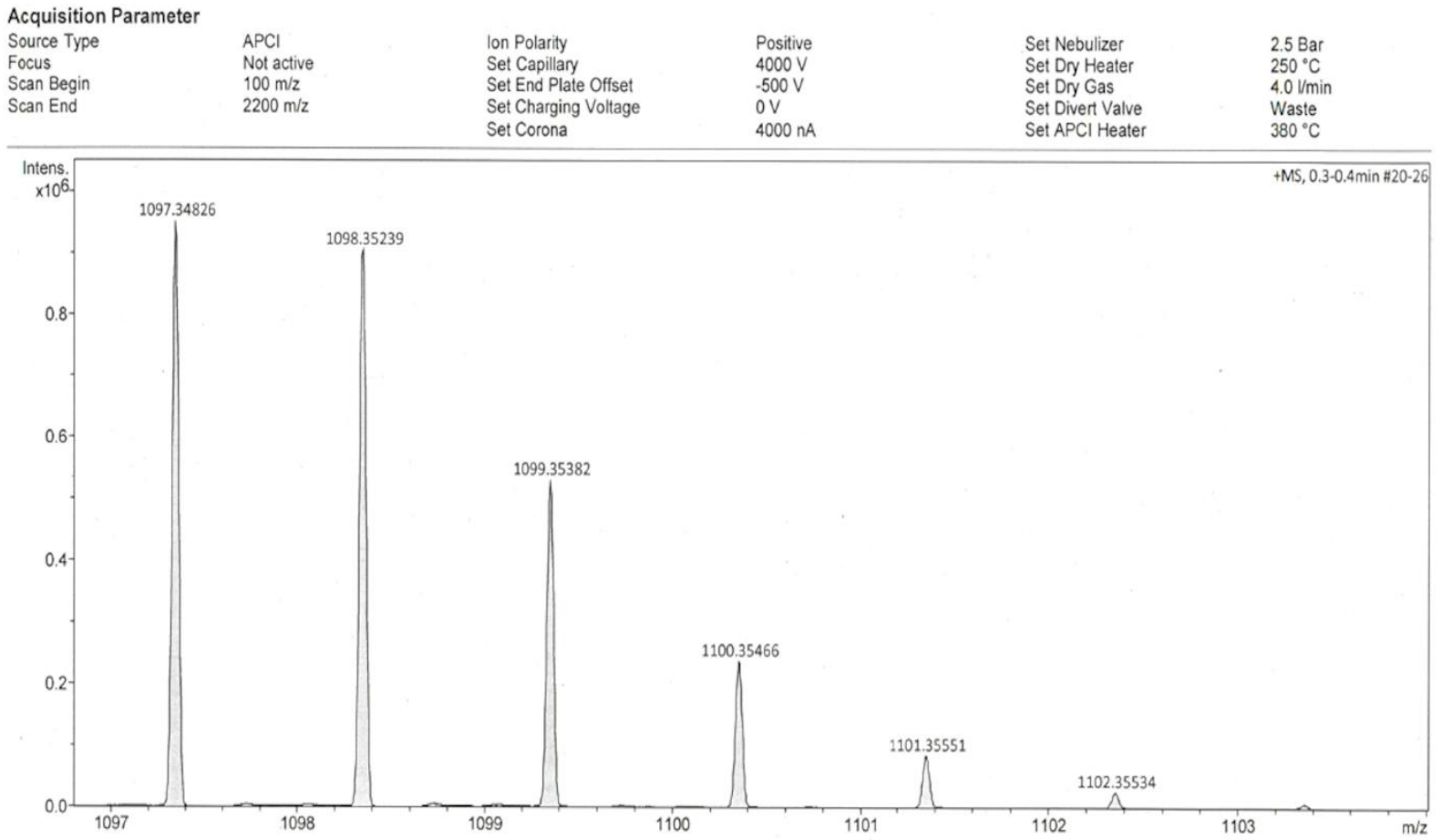

Figure S185. HRMS (APCI) of 23(S). 


\section{Computational Chemistry}

Table S2. HOMO-1, HOMO, LUMO and LUMO+1 energy levels of the optimized structures.

\begin{tabular}{|c|c|c|c|c|}
\hline Molecule & $\begin{array}{c}\text { HOMO -1 } \\
{[\mathbf{e V}]}\end{array}$ & $\begin{array}{c}\text { HOMO } \\
{[\mathbf{e V}]}\end{array}$ & $\begin{array}{c}\text { LUMO } \\
{[\mathbf{e V}]}\end{array}$ & $\begin{array}{c}\text { LUMO +1 } \\
{[\mathbf{e V}]}\end{array}$ \\
\hline $\mathbf{1 8}(\boldsymbol{S}) \mathbf{a}$ & -5.57 & -5.30 & -2.84 & -2.44 \\
\hline $\mathbf{1 8}(\boldsymbol{S}) \mathbf{b}$ & -5.30 & -4.76 & -2.71 & -2.35 \\
\hline $\mathbf{2 2}(\boldsymbol{S})$ & -5.53 & -5.24 & -3.22 & -2.39 \\
\hline
\end{tabular}

Table S 3. Number of imaginary frequencies and total energy of the optimized structures.

\begin{tabular}{|c|c|c|}
\hline Molecule & $\begin{array}{c}\text { Number of imaginary } \\
\text { frequencies }\end{array}$ & Total energy [Hartree] \\
\hline $\mathbf{1 8}(\boldsymbol{S}) \mathbf{a}$ & 0 & -3588.6 \\
\hline $\mathbf{1 8}(\boldsymbol{S}) \mathbf{b}$ & 0 & -4503.5 \\
\hline $\mathbf{2 2}(\boldsymbol{S})$ & 0 & -4216.6 \\
\hline
\end{tabular}



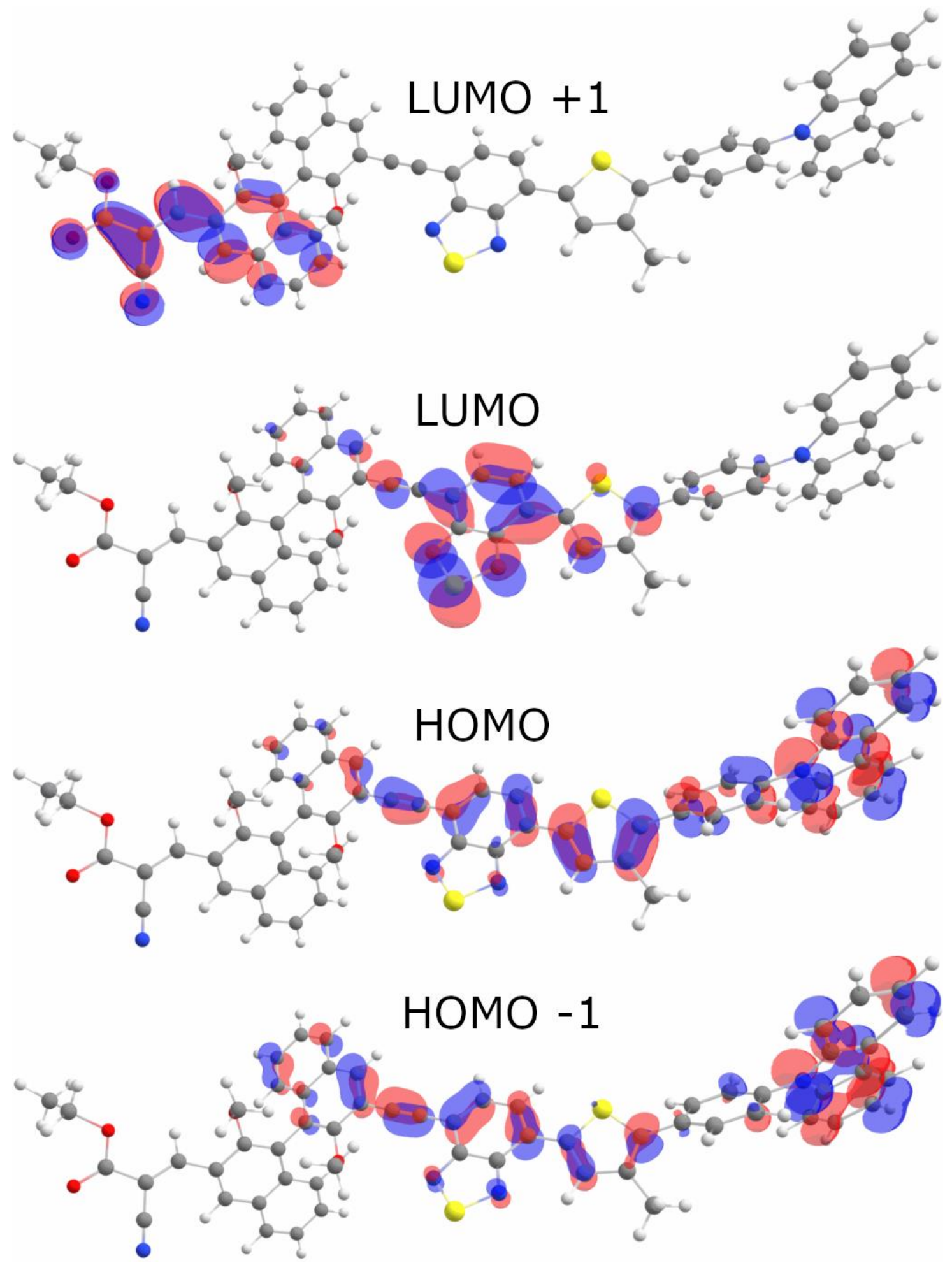

Figure S186. Frontier orbitals of $\mathbf{1 8}(S)$ a at B3LYP, 6-31G* level of theory and 0.03 contour value. 

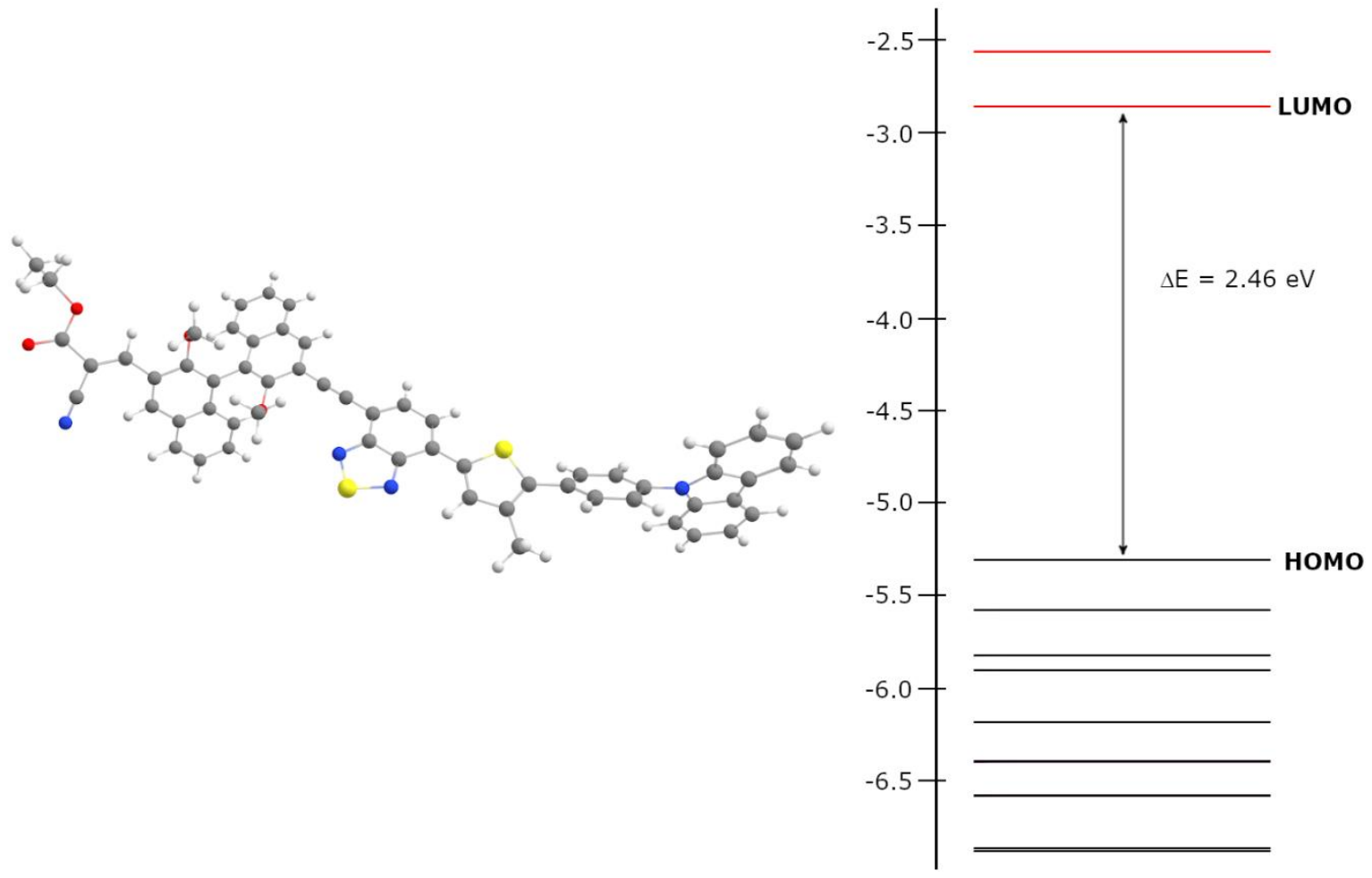

Figure S187. Optimized geometry and energy diagram, in eV, of $\mathbf{1 8}(\boldsymbol{S})$ a at B3LYP, 6-31G* level of theory.

Table S4. Cartesian coordinates of $\mathbf{1 8}(S)$ a optimized at B3LYP, 6-31G*, distances in angstrom.

\begin{tabular}{|l|c|c|c|}
\hline $\mathbf{C}$ & -13.076399 & 0.439691 & 0.774803 \\
\hline $\mathbf{C}$ & -12.813648 & 0.333325 & 2.143381 \\
\hline $\mathbf{H}$ & -11.828119 & 0.065857 & 2.509802 \\
\hline $\mathbf{C}$ & -13.864506 & 0.574102 & 3.0262 \\
\hline $\mathbf{H}$ & -13.683812 & 0.498881 & 4.095071 \\
\hline $\mathbf{C}$ & -15.149002 & 0.907032 & 2.56337 \\
\hline $\mathbf{H}$ & -15.946123 & 1.090621 & 3.278077 \\
\hline $\mathbf{C}$ & -15.40795 & 0.996865 & 1.198815 \\
\hline $\mathbf{H}$ & -16.404431 & 1.24351 & 0.841639 \\
\hline $\mathbf{C}$ & -14.37008 & 0.759366 & 0.290227 \\
\hline $\mathbf{C}$ & -14.292774 & 0.746993 & -1.155808 \\
\hline $\mathbf{H}$ & -15.220993 & 0.99516 & -2.173618 \\
\hline $\mathbf{C}$ & -16.249269 & 1.248444 & -1.928924 \\
\hline $\mathbf{H}$ & -14.808966 & 0.919691 & -3.500895 \\
\hline $\mathbf{C}$ & -15.520659 & 1.108867 & -4.299408 \\
\hline $\mathbf{H}$ & -13.476182 & 0.6068 & -3.818317 \\
\hline $\mathbf{C}$ & -13.170537 & 0.562705 & -4.860158 \\
\hline $\mathbf{H}$ & -12.532333 & 0.354787 & -2.824701 \\
\hline $\mathbf{C}$ & -11.502847 & 0.125762 & -3.079179 \\
\hline $\mathbf{C}$ & -12.955854 & 0.417207 & -1.49424 \\
\hline & -10.846881 & -0.10906 & -0.238739 \\
\hline & -10.35572 & -1.233947 & -0.913674 \\
\hline
\end{tabular}




\begin{tabular}{|c|c|c|c|}
\hline $\mathbf{H}$ & -11.037225 & -1.850413 & -1.491252 \\
\hline $\mathbf{C}$ & -9.004388 & -1.559499 & -0.846919 \\
\hline $\mathbf{H}$ & -8.637842 & -2.413068 & -1.407092 \\
\hline $\mathbf{C}$ & -8.106198 & -0.792759 & -0.080952 \\
\hline $\mathbf{C}$ & -8.616889 & 0.331791 & 0.595365 \\
\hline $\mathbf{H}$ & -7.949809 & 0.940081 & 1.199256 \\
\hline $\mathbf{C}$ & -9.961266 & 0.676889 & 0.510722 \\
\hline $\mathbf{H}$ & -10.331895 & 1.559333 & 1.022512 \\
\hline $\mathbf{C}$ & -6.680916 & -1.141968 & -0.004934 \\
\hline $\mathbf{C}$ & -6.075519 & -2.38297 & 0.099186 \\
\hline $\mathbf{C}$ & -4.656054 & -2.290148 & 0.151349 \\
\hline $\mathbf{H}$ & -4.011518 & -3.154226 & 0.244874 \\
\hline $\mathbf{C}$ & -4.153818 & -1.006089 & 0.09207 \\
\hline C & -6.791457 & -3.706275 & 0.199889 \\
\hline $\mathbf{H}$ & -7.799066 & -3.594252 & 0.609916 \\
\hline $\mathbf{H}$ & -6.235138 & -4.391702 & 0.848045 \\
\hline $\mathbf{H}$ & -6.884654 & -4.194993 & -0.779125 \\
\hline $\mathbf{C}$ & -2.773515 & -0.54626 & 0.129591 \\
\hline C & -2.43973 & 0.80227 & 0.172874 \\
\hline C & -1.113292 & 1.288919 & 0.203209 \\
\hline $\mathbf{C}$ & -0.005235 & 0.453523 & 0.196758 \\
\hline $\mathbf{C}$ & -0.282696 & -0.958123 & 0.14977 \\
\hline $\mathbf{C}$ & -1.650041 & -1.456089 & 0.1183 \\
\hline C & 1.320553 & 0.943006 & 0.233294 \\
\hline $\mathbf{C}$ & 2.462454 & 1.364585 & 0.268699 \\
\hline C & 3.783289 & 1.887167 & 0.297627 \\
\hline $\mathbf{C}$ & 4.003632 & 3.238633 & 0.074003 \\
\hline $\mathbf{H}$ & 3.157541 & 3.881086 & -0.152869 \\
\hline C & 5.295711 & 3.80257 & 0.160114 \\
\hline $\mathbf{C}$ & 5.505541 & 5.194227 & -0.044799 \\
\hline $\mathbf{H}$ & 4.648957 & 5.817521 & -0.290065 \\
\hline $\mathbf{C}$ & 6.760985 & 5.742638 & 0.071148 \\
\hline $\mathbf{H}$ & 6.911047 & 6.80729 & -0.084681 \\
\hline $\mathbf{C}$ & 7.86087 & 4.915764 & 0.401741 \\
\hline $\mathbf{H}$ & 8.85062 & 5.353061 & 0.501043 \\
\hline $\mathbf{C}$ & 7.690706 & 3.562949 & 0.599641 \\
\hline $\mathbf{H}$ & 8.543171 & 2.943347 & 0.853599 \\
\hline $\mathbf{C}$ & 6.410548 & 2.959544 & 0.480511 \\
\hline $\mathbf{C}$ & 6.192984 & 1.55314 & 0.67805 \\
\hline $\mathbf{C}$ & 4.906492 & 1.040407 & 0.598744 \\
\hline $\mathbf{C}$ & 4.327899 & -1.169821 & -0.137425 \\
\hline $\mathbf{H}$ & 3.468616 & -0.802664 & -0.704551 \\
\hline $\mathbf{H}$ & 4.053714 & -2.107817 & 0.347573 \\
\hline $\mathbf{H}$ & 5.180375 & -1.339912 & -0.806956 \\
\hline C & 7.33435 & 0.633489 & 0.989559 \\
\hline C & 7.476528 & 0.044597 & 2.291875 \\
\hline
\end{tabular}




\begin{tabular}{|c|c|c|c|}
\hline $\mathbf{C}$ & 6.582449 & 0.318149 & 3.359805 \\
\hline $\mathbf{H}$ & 5.752291 & 0.994673 & 3.193193 \\
\hline $\mathbf{C}$ & 6.757572 & -0.263208 & 4.596624 \\
\hline $\mathbf{H}$ & 6.059873 & -0.037538 & 5.398525 \\
\hline $\mathbf{C}$ & 7.833133 & -1.152056 & 4.836778 \\
\hline H & 7.953192 & -1.604518 & 5.816972 \\
\hline $\mathbf{C}$ & 8.72268 & -1.433885 & 3.828032 \\
\hline $\mathbf{H}$ & 9.55774 & -2.109488 & 3.994693 \\
\hline $\mathbf{C}$ & 8.573229 & -0.84502 & 2.54066 \\
\hline $\mathbf{C}$ & 9.504651 & -1.112658 & 1.515216 \\
\hline $\mathbf{H}$ & 10.333708 & -1.773433 & 1.738689 \\
\hline $\mathbf{C}$ & 9.395797 & -0.526732 & 0.260138 \\
\hline $\mathbf{C}$ & 8.282616 & 0.356126 & 0.020153 \\
\hline $\mathbf{C}$ & 7.473036 & 0.313629 & -2.222715 \\
\hline $\mathbf{H}$ & 6.408443 & 0.273143 & -1.967957 \\
\hline $\mathbf{H}$ & 7.850086 & -0.70451 & -2.385409 \\
\hline $\mathbf{H}$ & 7.604815 & 0.900043 & -3.134532 \\
\hline $\mathbf{C}$ & 10.361399 & -0.694869 & -0.81366 \\
\hline $\mathbf{H}$ & 10.253756 & 0.021099 & -1.620602 \\
\hline $\mathbf{N}$ & -12.220916 & 0.231988 & -0.315519 \\
\hline $\mathbf{O}$ & 4.69194 & -0.274612 & 0.919217 \\
\hline $\mathbf{O}$ & 8.21805 & 0.987133 & -1.205511 \\
\hline $\mathbf{C}$ & 11.373536 & -1.593286 & -0.979061 \\
\hline $\mathbf{S}$ & -5.482307 & 0.13647 & -0.045664 \\
\hline $\mathbf{N}$ & -1.715013 & -2.790869 & 0.073043 \\
\hline $\mathbf{S}$ & -0.168926 & -3.347707 & 0.070035 \\
\hline $\mathbf{N}$ & 0.642162 & -1.920122 & 0.124428 \\
\hline $\mathbf{H}$ & -0.955697 & 2.362132 & 0.239524 \\
\hline $\mathbf{H}$ & -3.236171 & 1.54046 & 0.191717 \\
\hline $\mathbf{C}$ & 11.67989 & -2.647112 & -0.060688 \\
\hline $\mathbf{C}$ & 12.265478 & -1.55986 & -2.178142 \\
\hline $\mathbf{N}$ & 11.916535 & -3.498244 & 0.697222 \\
\hline $\mathbf{O}$ & 13.174853 & -2.344877 & -2.353924 \\
\hline $\mathbf{O}$ & 11.939394 & -0.568755 & -3.037254 \\
\hline $\mathbf{C}$ & 12.760696 & -0.453205 & -4.225807 \\
\hline $\mathbf{H}$ & 13.013462 & -1.457032 & -4.574786 \\
\hline $\mathbf{H}$ & 12.107068 & 0.032437 & -4.9545 \\
\hline $\mathbf{C}$ & 14.010662 & 0.37078 & -3.958227 \\
\hline $\mathbf{H}$ & 14.65923 & -0.135808 & -3.238258 \\
\hline $\mathbf{H}$ & 14.571307 & 0.504927 & -4.890497 \\
\hline $\mathbf{H}$ & 13.74989 & 1.36002 & -3.568134 \\
\hline
\end{tabular}



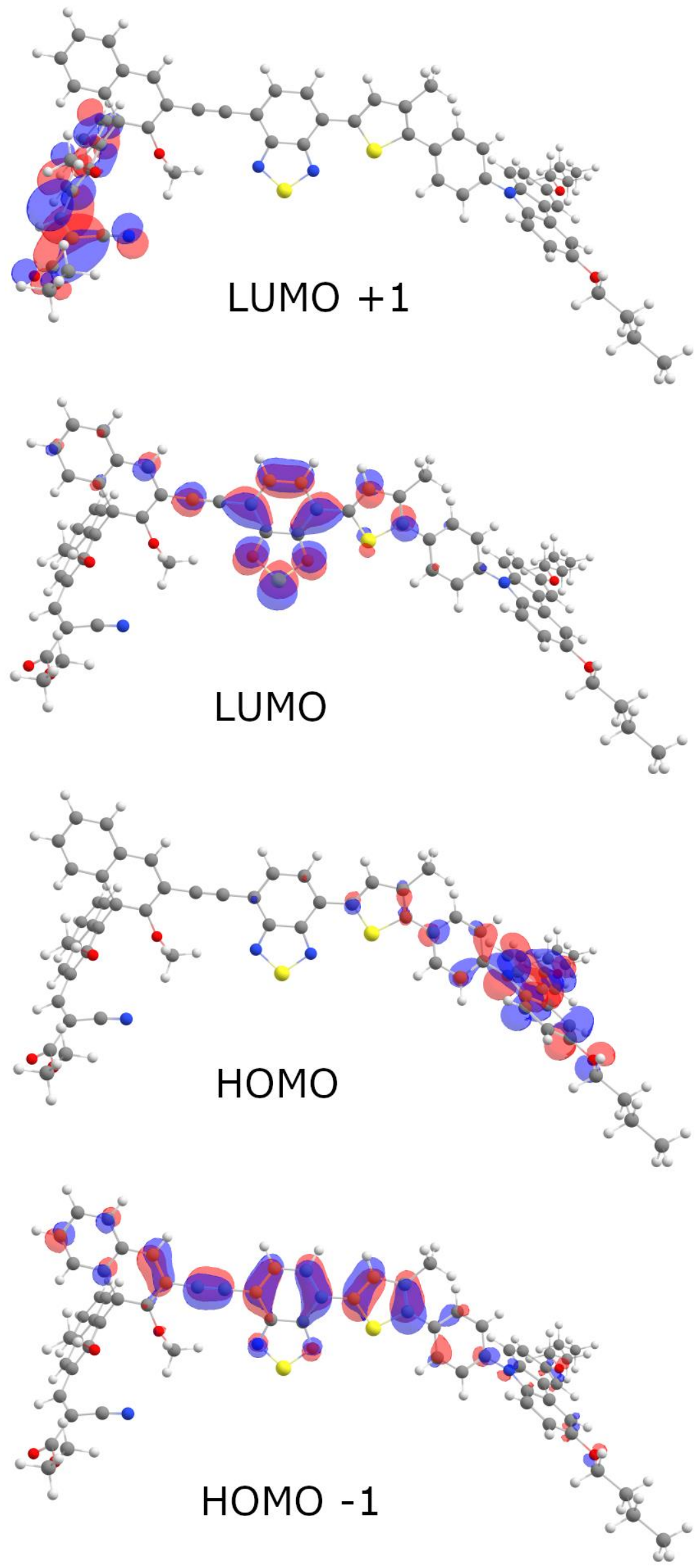

Figure S188. Frontier orbitals of $\mathbf{1 8}(\boldsymbol{S}) \mathbf{b}$ at B3LYP, 6-31G* level of theory and 0.03 contour value. 


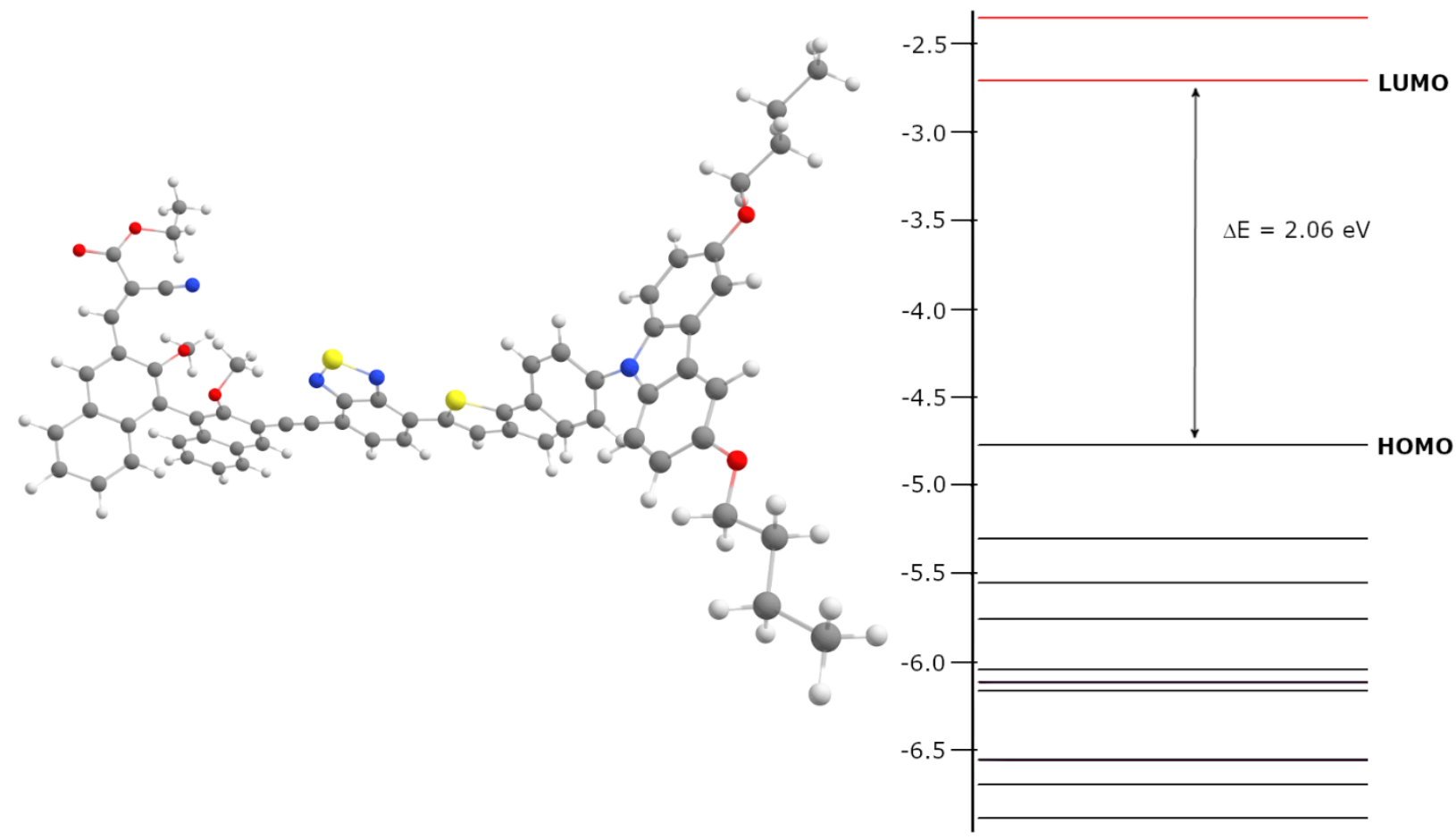

Figure S189. Optimized geometry and energy diagram, in eV, of $\mathbf{1 8}(\boldsymbol{S}) \mathbf{b}$ at B3LYP, 6-31G* level of theory.

Table S5. Cartesian coordinates of $\mathbf{1 8}(S)$ b optimized at B3LYP, 6-31G*, distances in angstrom.

\begin{tabular}{|l|c|c|c|}
\hline $\mathbf{C}$ & -11.650983 & -3.287704 & -0.141183 \\
\hline $\mathbf{C}$ & -12.017073 & -2.053502 & 0.402406 \\
\hline $\mathbf{C}$ & -11.122266 & -0.986244 & 0.332487 \\
\hline $\mathbf{C}$ & -9.853845 & -1.158726 & -0.278506 \\
\hline $\mathbf{C}$ & -9.493556 & -2.382771 & -0.837884 \\
\hline $\mathbf{C}$ & -10.397158 & -3.445197 & -0.762051 \\
\hline $\mathbf{C}$ & -11.196694 & 0.395514 & 0.764259 \\
\hline $\mathbf{C}$ & -9.972222 & 1.008703 & 0.394637 \\
\hline $\mathbf{N}$ & -9.154886 & 0.059151 & -0.240618 \\
\hline $\mathbf{C}$ & -12.17449 & 1.130938 & 1.433364 \\
\hline $\mathbf{C}$ & -11.929477 & 2.472238 & 1.739373 \\
\hline $\mathbf{C}$ & -10.704002 & 3.068007 & 1.38492 \\
\hline $\mathbf{C}$ & -9.717747 & 2.340539 & 0.714815 \\
\hline $\mathbf{C}$ & -7.857504 & 0.288826 & -0.752945 \\
\hline $\mathbf{C}$ & -6.794973 & -0.56044 & -0.41089 \\
\hline $\mathbf{C}$ & -5.523485 & -0.340338 & -0.928213 \\
\hline $\mathbf{C}$ & -5.258367 & 0.745451 & -1.785215 \\
\hline $\mathbf{C}$ & -6.329862 & 1.601545 & -2.10256 \\
\hline $\mathbf{C}$ & -7.610216 & 1.371084 & -1.60927 \\
\hline $\mathbf{C}$ & -3.907204 & 0.98648 & -2.310252 \\
\hline $\mathbf{C}$ & -3.512992 & 1.39666 & -3.57554 \\
\hline & -2.10151 & 1.493483 & -3.682157 \\
\hline & & & \\
\hline
\end{tabular}




\begin{tabular}{|c|c|c|c|}
\hline $\mathbf{C}$ & -1.399223 & 1.173081 & -2.534272 \\
\hline $\mathbf{S}$ & -2.525799 & 0.722007 & -1.269074 \\
\hline $\mathbf{C}$ & -4.428525 & 1.662667 & -4.743866 \\
\hline $\mathbf{O}$ & -12.583347 & -4.286149 & -0.026152 \\
\hline $\mathbf{O}$ & -12.942271 & 3.125957 & 2.392346 \\
\hline $\mathbf{C}$ & -12.758692 & 4.488882 & 2.758757 \\
\hline $\mathbf{C}$ & -14.023035 & 4.953819 & 3.469445 \\
\hline C & -13.945554 & 6.419524 & 3.915052 \\
\hline $\mathbf{C}$ & -15.215727 & 6.890192 & 4.630914 \\
\hline C & -12.286843 & -5.570654 & -0.562549 \\
\hline C & -13.482648 & -6.474686 & -0.293112 \\
\hline $\mathbf{C}$ & -13.280169 & -7.897626 & -0.828931 \\
\hline $\mathbf{C}$ & -14.481095 & -8.809978 & -0.559277 \\
\hline $\mathbf{C}$ & 2.156205 & 0.707122 & -1.063652 \\
\hline $\mathbf{C}$ & 0.710405 & 0.661931 & -1.199718 \\
\hline $\mathbf{C}$ & 0.045311 & 1.21423 & -2.352588 \\
\hline C & 0.887462 & 1.795449 & -3.294745 \\
\hline $\mathbf{C}$ & 2.294465 & 1.837356 & -3.171156 \\
\hline C & 2.975418 & 1.310739 & -2.080441 \\
\hline $\mathbf{N}$ & 2.60584 & 0.148824 & 0.06248 \\
\hline $\mathbf{S}$ & 1.285773 & -0.3863 & 0.882167 \\
\hline $\mathbf{N}$ & 0.105539 & 0.069931 & -0.166134 \\
\hline $\mathrm{C}$ & 11.863493 & 2.690712 & -2.765398 \\
\hline C & 11.134733 & 3.025643 & -3.932113 \\
\hline $\mathbf{C}$ & 9.775784 & 2.818675 & -3.970026 \\
\hline C & 9.087534 & 2.271596 & -2.852102 \\
\hline $\mathbf{C}$ & 9.820732 & 1.936999 & -1.665175 \\
\hline $\mathbf{C}$ & 11.226082 & 2.162658 & -1.663541 \\
\hline C & 7.69338 & 2.058042 & -2.878216 \\
\hline $\mathbf{C}$ & 7.010434 & 1.520936 & -1.793444 \\
\hline $\mathbf{C}$ & 7.753493 & 1.152312 & -0.615915 \\
\hline $\mathbf{C}$ & 9.124383 & 1.36886 & -0.550474 \\
\hline $\mathbf{C}$ & 9.855444 & 0.992021 & 0.700661 \\
\hline $\mathbf{C}$ & 10.123068 & -0.340169 & 0.966351 \\
\hline $\mathbf{C}$ & 10.836065 & -0.74461 & 2.142697 \\
\hline $\mathbf{C}$ & 11.320583 & 0.242428 & 2.992008 \\
\hline $\mathbf{C}$ & 11.04305 & 1.609946 & 2.782761 \\
\hline $\mathbf{C}$ & 10.280244 & 1.996581 & 1.631772 \\
\hline $\mathbf{C}$ & 11.493074 & 2.606077 & 3.692919 \\
\hline $\mathbf{C}$ & 11.195994 & 3.932373 & 3.486415 \\
\hline $\mathbf{C}$ & 10.430701 & 4.315553 & 2.358344 \\
\hline $\mathbf{C}$ & 9.986034 & 3.375372 & 1.454729 \\
\hline $\mathbf{O}$ & 7.147098 & 0.656685 & 0.490611 \\
\hline $\mathbf{O}$ & 9.700998 & -1.308748 & 0.085736 \\
\hline $\mathbf{C}$ & 10.658038 & -1.679469 & -0.910513 \\
\hline C & 6.308828 & -0.512934 & 0.373842 \\
\hline
\end{tabular}




\begin{tabular}{|c|c|c|c|}
\hline $\mathbf{C}$ & 11.115875 & -2.126329 & 2.5168 \\
\hline $\mathbf{C}$ & 10.392333 & -3.269946 & 2.373602 \\
\hline C & 5.594904 & 1.415362 & -1.853271 \\
\hline $\mathbf{C}$ & 4.382844 & 1.365263 & -1.961729 \\
\hline $\mathbf{C}$ & 9.039561 & -3.3143 & 1.919439 \\
\hline C & 11.013298 & -4.526097 & 2.954672 \\
\hline $\mathbf{N}$ & 7.913457 & -3.421238 & 1.642111 \\
\hline $\mathbf{O}$ & 11.848426 & -4.465719 & 3.82972 \\
\hline $\mathbf{O}$ & 10.617978 & -5.735304 & 2.507122 \\
\hline H & -12.994599 & -1.951342 & 0.863393 \\
\hline $\mathbf{H}$ & -8.537514 & -2.519899 & -1.331796 \\
\hline $\mathbf{H}$ & -10.115172 & -4.397691 & -1.194947 \\
\hline H & -13.120286 & 0.688355 & 1.73015 \\
\hline $\mathbf{H}$ & -10.506025 & 4.104279 & 1.63217 \\
\hline H & -8.773958 & 2.812689 & 0.463629 \\
\hline H & -6.970689 & -1.389028 & 0.267568 \\
\hline H & -4.71964 & -1.022414 & -0.666774 \\
\hline $\mathbf{H}$ & -6.155653 & 2.466182 & -2.734429 \\
\hline $\mathbf{H}$ & -8.427205 & 2.028884 & -1.888001 \\
\hline $\mathbf{H}$ & -1.615416 & 1.768225 & -4.612614 \\
\hline $\mathbf{H}$ & -5.359488 & 1.093843 & -4.66672 \\
\hline H & -3.940042 & 1.386339 & -5.684683 \\
\hline $\mathbf{H}$ & -4.697318 & 2.724733 & -4.821127 \\
\hline $\mathbf{H}$ & -12.571504 & 5.100215 & 1.862488 \\
\hline $\mathbf{H}$ & -11.883937 & 4.588791 & 3.419578 \\
\hline $\mathbf{H}$ & -14.195803 & 4.304824 & 4.337781 \\
\hline $\mathbf{H}$ & -14.877295 & 4.809794 & 2.795198 \\
\hline $\mathbf{H}$ & -13.761488 & 7.059221 & 3.040235 \\
\hline $\mathbf{H}$ & -13.080663 & 6.555111 & 4.579808 \\
\hline $\mathbf{H}$ & -16.093546 & 6.799874 & 3.979735 \\
\hline $\mathbf{H}$ & -15.134277 & 7.938784 & 4.938227 \\
\hline $\mathbf{H}$ & -15.407763 & 6.29232 & 5.530016 \\
\hline $\mathbf{H}$ & -11.379563 & -5.977923 & -0.090418 \\
\hline $\mathbf{H}$ & -12.094945 & -5.494571 & -1.643927 \\
\hline $\mathbf{H}$ & -14.373213 & -6.023459 & -0.749596 \\
\hline $\mathbf{H}$ & -13.664485 & -6.502064 & 0.789133 \\
\hline $\mathbf{H}$ & -12.380101 & -8.335565 & -0.374583 \\
\hline $\mathbf{H}$ & -13.087763 & -7.857939 & -1.910493 \\
\hline $\mathbf{H}$ & -14.677472 & -8.898135 & 0.516079 \\
\hline $\mathbf{H}$ & -14.310697 & -9.818829 & -0.951456 \\
\hline $\mathbf{H}$ & -15.390154 & -8.417237 & -1.030566 \\
\hline
\end{tabular}



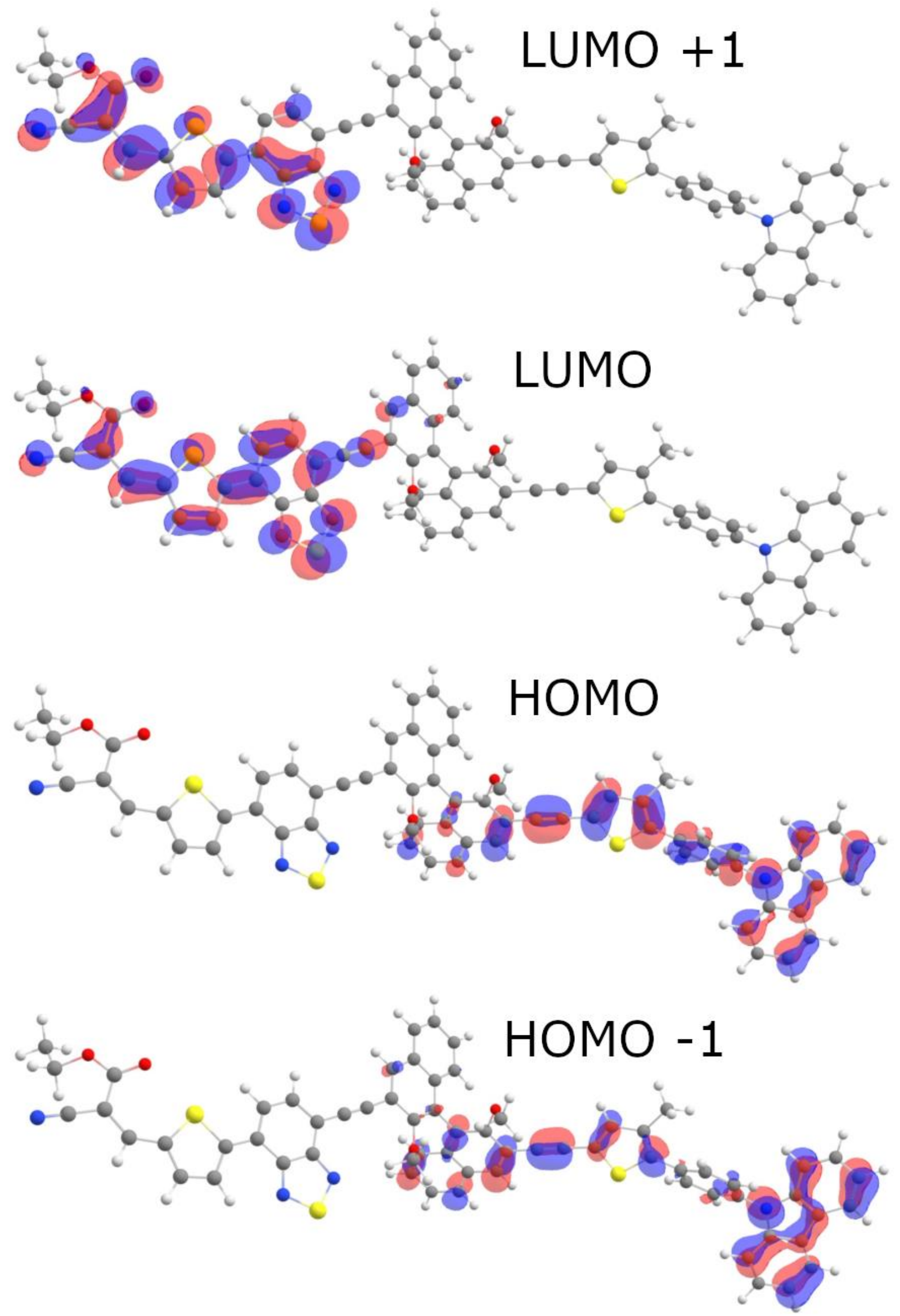

Figure S190. Frontier orbitals of $22(S)$ at B3LYP, 6-31G* level of theory and 0.03 contour value. 

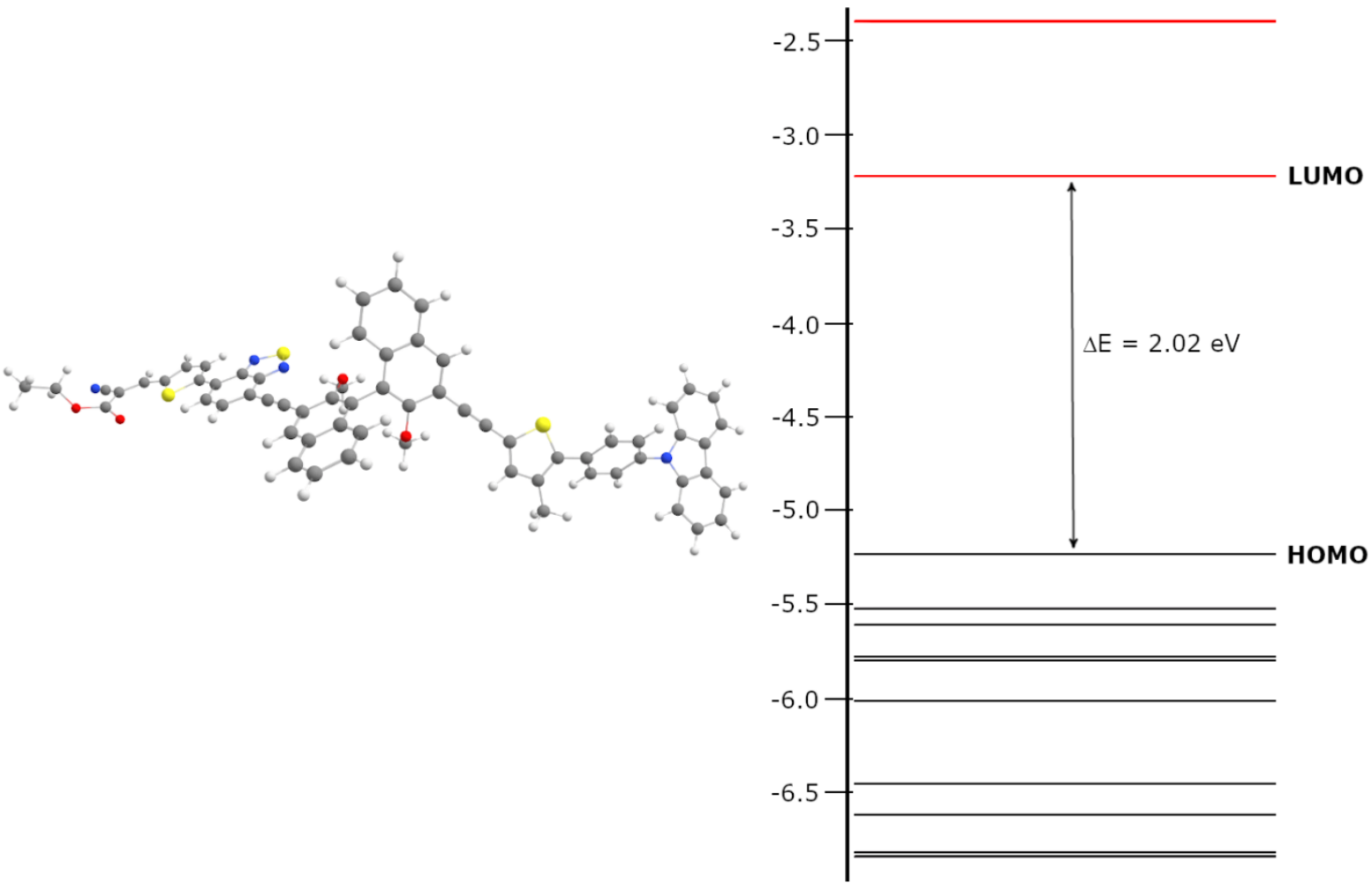

Figure S191. Optimized geometry and energy diagram, in eV, of $22(S)$ at B3LYP, 6-31G* level of theory.

Table S6. Cartesian coordinates of $\mathbf{2 2}(S)$ optimized at B3LYP, 6-31G*, distances in angstrom.

\begin{tabular}{|l|c|c|c|}
\hline $\mathbf{C}$ & -16.776986 & -2.527563 & -2.81604 \\
\hline $\mathbf{C}$ & -16.890109 & -2.696943 & -1.43921 \\
\hline $\mathbf{C}$ & -15.778973 & -2.443058 & -0.626784 \\
\hline $\mathbf{C}$ & -14.560113 & -2.026278 & -1.219684 \\
\hline $\mathbf{C}$ & -14.444566 & -1.840234 & -2.600059 \\
\hline $\mathbf{C}$ & -15.565819 & -2.099152 & -3.385758 \\
\hline $\mathbf{C}$ & -15.555159 & -2.494866 & 0.802982 \\
\hline $\mathbf{C}$ & -14.209876 & -2.104771 & 1.023793 \\
\hline $\mathbf{C}$ & -13.609924 & -1.821641 & -0.210506 \\
\hline $\mathbf{C}$ & -16.3602 & -2.844193 & 1.89343 \\
\hline $\mathbf{C}$ & -15.819217 & -2.808037 & 3.175248 \\
\hline $\mathbf{C}$ & -14.479292 & -2.433927 & 3.374705 \\
\hline $\mathbf{C}$ & -13.656518 & -2.08094 & 2.307019 \\
\hline $\mathbf{C}$ & -12.270992 & -1.397659 & -0.405 \\
\hline $\mathbf{C}$ & -11.426813 & -2.092467 & -1.280539 \\
\hline $\mathbf{C}$ & -10.114633 & -1.671518 & -1.474488 \\
\hline $\mathbf{C}$ & -9.600584 & -0.548899 & -0.798542 \\
\hline $\mathbf{C}$ & -10.458433 & 0.130479 & 0.087805 \\
\hline $\mathbf{C}$ & -11.773171 & -0.280138 & 0.278488 \\
\hline & -8.21122 & -0.111123 & -0.993737 \\
\hline & -7.457547 & -0.04864 & -2.155912 \\
\hline
\end{tabular}




\begin{tabular}{|c|c|c|c|}
\hline $\mathbf{C}$ & -6.144083 & 0.445796 & -1.922576 \\
\hline $\mathbf{C}$ & -5.875426 & 0.763703 & -0.606152 \\
\hline $\mathbf{S}$ & -7.283822 & 0.439319 & 0.389224 \\
\hline $\mathbf{C}$ & -4.691159 & 1.273136 & -0.049878 \\
\hline $\mathbf{C}$ & -3.669643 & 1.717597 & 0.444117 \\
\hline $\mathbf{C}$ & 0.262466 & 5.995123 & -2.229017 \\
\hline $\mathbf{C}$ & 1.425667 & 6.077088 & -3.032952 \\
\hline $\mathbf{C}$ & 2.453057 & 5.185122 & -2.839013 \\
\hline $\mathbf{C}$ & 2.362499 & 4.176505 & -1.839509 \\
\hline $\mathbf{C}$ & 1.18345 & 4.086715 & -1.027998 \\
\hline $\mathbf{C}$ & 0.143722 & 5.028552 & -1.255048 \\
\hline $\mathbf{C}$ & 3.418952 & 3.271731 & -1.613146 \\
\hline $\mathbf{C}$ & 3.348976 & 2.290507 & -0.629464 \\
\hline $\mathbf{C}$ & 2.14736 & 2.177956 & 0.155575 \\
\hline $\mathbf{C}$ & 1.09029 & 3.058809 & -0.035084 \\
\hline $\mathbf{C}$ & -0.137667 & 2.925547 & 0.812844 \\
\hline $\mathbf{C}$ & -1.285733 & 2.379681 & 0.268623 \\
\hline $\mathbf{C}$ & -2.490836 & 2.242764 & 1.038407 \\
\hline $\mathbf{C}$ & -2.490959 & 2.660021 & 2.361483 \\
\hline $\mathbf{C}$ & -1.340545 & 3.226842 & 2.955423 \\
\hline $\mathbf{C}$ & -0.146358 & 3.375931 & 2.175716 \\
\hline $\mathbf{C}$ & -1.349816 & 3.667005 & 4.307596 \\
\hline C & -0.232292 & 4.235916 & 4.872416 \\
\hline $\mathbf{C}$ & 0.945108 & 4.390144 & 4.102707 \\
\hline $\mathbf{C}$ & 0.986602 & 3.971185 & 2.790671 \\
\hline $\mathbf{O}$ & 2.019833 & 1.266916 & 1.161676 \\
\hline $\mathbf{O}$ & -1.304157 & 2.018811 & -1.058284 \\
\hline $\mathbf{C}$ & -1.171931 & 0.619597 & -1.316388 \\
\hline $\mathbf{C}$ & 2.311632 & -0.117045 & 0.920483 \\
\hline $\mathbf{C}$ & 4.492616 & 1.482883 & -0.393435 \\
\hline $\mathbf{C}$ & 5.515436 & 0.838945 & -0.237403 \\
\hline $\mathbf{C}$ & 6.77087 & -0.985881 & 0.895129 \\
\hline $\mathbf{C}$ & 7.9991 & -1.739946 & 1.092282 \\
\hline $\mathbf{C}$ & 9.190967 & -1.423062 & 0.339356 \\
\hline $\mathbf{C}$ & 9.062427 & -0.366212 & -0.556222 \\
\hline $\mathbf{C}$ & 7.871956 & 0.367813 & -0.749505 \\
\hline $\mathbf{C}$ & 6.702802 & 0.099536 & -0.048848 \\
\hline $\mathbf{N}$ & 5.756384 & -1.399578 & 1.656702 \\
\hline $\mathbf{S}$ & 6.323725 & -2.650234 & 2.556889 \\
\hline $\mathbf{N}$ & 7.871123 & -2.706988 & 2.007086 \\
\hline C & 10.440718 & -2.153361 & 0.499735 \\
\hline $\mathbf{C}$ & 10.736066 & -3.180236 & 1.391225 \\
\hline $\mathbf{C}$ & 12.047455 & -3.653906 & 1.257833 \\
\hline $\mathbf{C}$ & 12.799518 & -3.010186 & 0.273818 \\
\hline $\mathbf{S}$ & 11.817289 & -1.795416 & -0.525617 \\
\hline C & 14.16722 & -3.355463 & 0.048655 \\
\hline
\end{tabular}




\begin{tabular}{|c|c|c|c|}
\hline $\mathbf{C}$ & 15.189593 & -2.823031 & -0.70068 \\
\hline $\mathbf{C}$ & 16.439261 & -3.515808 & -0.656546 \\
\hline $\mathbf{C}$ & 15.036868 & -1.651246 & -1.614619 \\
\hline $\mathbf{N}$ & 17.452471 & -4.091438 & -0.632189 \\
\hline $\mathbf{O}$ & 13.972665 & -1.396104 & -2.143519 \\
\hline $\mathbf{O}$ & 16.101321 & -0.8743 & -1.895597 \\
\hline $\mathbf{H}$ & -17.632118 & -2.722837 & -3.456791 \\
\hline $\mathbf{H}$ & -17.830488 & -3.017619 & -0.998461 \\
\hline $\mathbf{H}$ & -13.516901 & -1.499134 & -3.047397 \\
\hline $\mathbf{H}$ & -15.499511 & -1.962784 & -4.461798 \\
\hline $\mathbf{H}$ & -17.39336 & -3.144473 & 1.739081 \\
\hline $\mathbf{H}$ & -16.434598 & -3.07559 & 4.029453 \\
\hline $\mathbf{H}$ & -14.07158 & -2.42143 & 4.381885 \\
\hline $\mathbf{H}$ & -12.620121 & -1.804485 & 2.469611 \\
\hline $\mathbf{H}$ & -11.795682 & -2.978757 & -1.787135 \\
\hline $\mathbf{H}$ & -9.466522 & -2.247023 & -2.126911 \\
\hline $\mathbf{H}$ & -10.097862 & 1.013198 & 0.608104 \\
\hline $\mathbf{H}$ & -12.428156 & 0.276546 & 0.941162 \\
\hline $\mathbf{C}$ & -7.943749 & -0.401778 & -3.539283 \\
\hline $\mathbf{H}$ & -5.411115 & 0.585678 & -2.709986 \\
\hline $\mathbf{H}$ & -0.546439 & 6.704137 & -2.383138 \\
\hline $\mathbf{H}$ & 1.502534 & 6.845258 & -3.797289 \\
\hline $\mathbf{H}$ & 3.354758 & 5.236957 & -3.444609 \\
\hline $\mathbf{H}$ & -0.753674 & 4.977219 & -0.648859 \\
\hline $\mathbf{H}$ & 4.325238 & 3.353232 & -2.206488 \\
\hline $\mathbf{H}$ & -3.399437 & 2.561761 & 2.948868 \\
\hline $\mathbf{H}$ & -2.262463 & 3.546568 & 4.88643 \\
\hline $\mathbf{H}$ & -0.250748 & 4.569496 & 5.906299 \\
\hline $\mathbf{H}$ & 1.825059 & 4.842919 & 4.55156 \\
\hline $\mathbf{H}$ & 1.894994 & 4.094249 & 2.211152 \\
\hline $\mathbf{H}$ & -1.222464 & 0.50574 & -2.401502 \\
\hline $\mathbf{H}$ & -1.983344 & 0.047743 & -0.85197 \\
\hline $\mathbf{H}$ & -0.205927 & 0.245066 & -0.955252 \\
\hline $\mathbf{H}$ & 1.603016 & -0.681598 & 1.53317 \\
\hline $\mathbf{H}$ & 3.333051 & -0.365141 & 1.215879 \\
\hline $\mathbf{H}$ & 2.162063 & -0.375274 & -0.134067 \\
\hline $\mathbf{H}$ & 9.922872 & -0.070335 & -1.149021 \\
\hline $\mathbf{H}$ & 7.872916 & 1.177527 & -1.471735 \\
\hline $\mathbf{H}$ & 10.016638 & -3.562639 & 2.101166 \\
\hline $\mathbf{H}$ & 12.465635 & -4.446139 & 1.870875 \\
\hline $\mathbf{H}$ & 14.458416 & -4.22096 & 0.642325 \\
\hline $\mathbf{C}$ & 17.264289 & -0.747955 & -1.038855 \\
\hline $\mathbf{H}$ & -9.029617 & -0.305183 & -3.624927 \\
\hline $\mathbf{H}$ & -7.680363 & -1.431658 & -3.814755 \\
\hline $\mathbf{H}$ & -7.485221 & 0.256932 & -4.284705 \\
\hline $\mathbf{C}$ & 17.777713 & 0.673096 & -1.192035 \\
\hline
\end{tabular}




\begin{tabular}{|l|l|l|l|}
\hline $\mathbf{H}$ & 18.013607 & -1.480194 & -1.349637 \\
\hline $\mathbf{H}$ & 16.983969 & -0.957962 & -0.001489 \\
\hline $\mathbf{H}$ & 18.031277 & 0.880329 & -2.236108 \\
\hline $\mathbf{H}$ & 18.678767 & 0.807516 & -0.583341 \\
\hline $\mathbf{H}$ & 17.024714 & 1.398181 & -0.868351 \\
\hline
\end{tabular}




\section{References}

[1] Fulmer, G. R.; Miller, A. J. M.; Sherden, N. H.; Gottlieb, H. E.; Nudelman, A.; Stoltz, B. M.; Bercaw, J. E.; Goldberg, K. I. NMR Chemical Shifts of Trace Impurities: Common Laboratory Solvents, Organics, and Gases in Deuterated Solvents Relevant to the Organometallic Chemist. Organometallics 2010, 29 (9), 2176-2179.

[2] Huq, M. M.; Robiur Rahman, M.; Naher, M.; Khan, M. M. R.; Masud, M. K.; Hossain, G. M. G.; Zhu, N.; Lo, Y. H.; Younus, M.; Wong, W.-Y. Synthesis, Characterization and Catalytic Activities of Palladium(II) Nitroaryl Complexes. J. Inorg. Organomet. P. 2016, 26 (6), 1243-1252.

[3] Guthrie, D. A.; Tovar, J. D. Conformation as a Protecting Group: A Regioselective Aromatic Bromination En Route to Complex $\pi$-Electron Systems. Org. Lett. 2008, 10 (19), 4323-4326.

[4] Imit, M.; Imin, P.; Adronov, A. Supramolecular Interactions of Fluorene-Based Copolymers Containing 3,4-Propylenedioxythiophene and Phenazine Units with SWNTs. Polym. Chem. 2016, 7 (33), 5241-5248.

[5] Limberg, F. R. P.; Miasojedovas, A.; Pingel, P.; Reisbeck, F.; Janietz, S.; Monkman, A. P.; Krüger, H. Hole-Transporting Side-Chain Polystyrenes Based on TCTA With Tuned Glass Transition and Optimized Electronic Properties. RSC Adv. 2015, 5 (101), 83122-83128.

[6] Dall'Agnese, C.; Hernández Maldonado, D.; Le Borgne, D.; Moineau-Chane Ching, K. I. Dissymmetrization of Benzothiadiazole by Direct $\mathrm{C}-\mathrm{H}$ Arylation: A Way to Symmetrical and Unsymmetrical Elongated $\pi$-Conjugated Molecules. Eur. J. Org. Chem. 2017, 2017 (46), 6872-6877.

[7] Brenet, S.; Baptiste, B.; Philouze, C.; Berthiol, F.; Einhorn, J. BINOL-Fused Maleimides - A New Class of C2-Symmetric Chiral Imides. Eur. J. Org. Chem. 2013, 2013 (6), 1041-1045.

[8] Wipf, P.; Jung, J.-K. Formal Total Synthesis of (+)-Diepoxin o. J. Org. Chem. 2000, 65 (20), $6319-6337$.

[9] Mosca, D.; Stopin, A.; Wouters, J.; Demitri, N.; Bonifazi, D. Stereospecific Winding of Polycyclic Aromatic Hydrocarbons into Trinacria Propellers. Chem. Eur. J. 2017, 23 (61), 15348-15354.

[10] Parker, C. A.; Rees, W. T. Correction of Fluorescence Spectra and Measurement of Fluorescence Quantum Efficiency. Analyst 1960, 85 (1013), 587-600.

[11] Melhuish, W. H. Quantum Efficiencies of Fluorescence of Organic Substances: Effect of Solvent and Concentration of the Fluorescent Solute. J. Phys. Chem. 1961, 65 (2), 229-235.

[12] Jones, G.; Jackson, W. R.; Halpern, A. M. Medium Effects on Fluorescence Quantum Yields and Lifetimes for Coumarin Laser Dyes. Chem. Phys. Lett. 1980, 72 (2), 391-395.

[13] Nakamaru, K.; Nishio, K.; Nobe, H. Reexamination of the Luminescence Quantum Yields of Tris(2,2'bipyridyl or 1,10-phenanthroline and their Derivatives)Ruthenium(II) Ions in Aqueous Solutions at Room Temperature ( $\left.25^{\circ} \mathrm{C}\right)$. Sci. Rep. Hirosaki Univ. 1979, 26 (1), 57-62.

[14] Frisch, M. J.; Trucks, G. W.; Schlegel, H. B.; Scuseria, G. E.; Robb, M. A.; Cheeseman, J. R.; Scalmani, G.; Barone, V.; Petersson, G. A.; Nakatsuji, H.; Li, X.; Caricato, M.; Marenich, A. V.; Bloino, J.; Janesko, B. G.; Gomperts, R.; Mennucci, B.; Hratchian, H. P.; Ortiz, J. V.; Izmaylov, A. F.; Sonnenberg, J. L.; Williams-Young, D.; Ding, F.; Lipparinin, F.; Egidi, F.; Goings, J.; Peng, B.; Petrone, A.; Henderson, T.; Ranasinghe, D.; Zakrzewski, V. G.; Gao, J.; Rega, N.; Zheng, G.; Liang, W.; Hada, M.; Ehara, M.; Toyota, K.; Fukuda, R.; Hasegawa, J.; Ishida, M.; Nakajima, T.; Honda, Y.; Kitao, O.; Nakai, H.; Vreven, T.; Throssell, K.; Montgomery, J. A., Jr.; Peralta, J. E.; Ogliaro, F.; Bearpark, M. J.; Heyd, J. J.; Brothers, E. N.; Kudin, K. N.; Staroverov, V. N.; Keith, T. A.; Kobayashi, R.; Normand, J.; Raghavachari, K.; Rendell, A. P.; Burant, J. C.; Iyengar, S. S.; Tomasi, J.; Cossi, M.; Millam, J. M.; Klene, M.; Adamo, C.; Cammi, R.; Ochterski, J. W.; Martin, R. L.; Morokuma, K.; Farkas, O.; Foresman, J. B.; Fox, D. J. Gaussian 16, Revision C.01, Gaussian, Inc., Wallingford CT: 2019.

[15] Selected publications: (a) Becke, A. D. Density-Functional Thermochemistry. III. The Role of Exact Exchange. J. Chem. Phys. 1993, 98 (7), 5648-5652. (b) Lee, C.; Yang, W.; Parr, R. G. Development of the ColleSalvetti Correlation-Energy Formula into a Functional of the Electron Density. Phys. Rev. B 1988, 37 (2), $785-789$. [16] Selected publications: (a) Petersson, G. A.; Bennett, A.; Tensfeldt, T. G.; Al-Laham, M. A.; Shirley, W. A.; Mantzaris, J. A. Complete Basis Set Model Chemistry. I. The Total Energies of Closed-Shell Atoms and Hydrides of the First-Row Elements. J. Chem. Phys 1988, 89 (4), 2193-2218. (b) Petersson, G. A.; Al-Laham, M. A. A Complete Basis Set Model Chemistry. II. Open-Shell Systems and the Total Energies of the First-Row Atoms. J. Chem. Phys. 1991, 94 (9), 6081-6090.

[17] Matsumoto, N.; Shimosaka, T. Validation of a Quantitative Analytical Method Based on the Effective Magnetic Moment and the Curie-Weiss law. Accredit. Qual. Assur. 2015, 20 (2), 115-124. 